\title{
The role of DLG-MAGUKs \\ in mediating signaling specificity \\ at the postsynaptic density
}

\author{
Dissertation \\ for the award of the degree \\ "Doctor rerum naturalium" (Dr.rer.nat.) \\ of the Georg-August-Universität Göttingen
}

within the doctoral program Biology

of the Georg-August-University School of Science (GAUSS)

\author{
submitted by \\ Joana-Kristin Duda
}

from Holzminden, Germany

Göttingen, 2018 



\section{Thesis Committee}

\section{Prof. Dr. Siegrid Löwel}

Department of Systems Neuroscience, Johann-Friedrich-Blumenbach-Institute of Zoology and Anthropology, University of Göttingen

\section{Prof. Dr. Dr. Oliver Schlüter}

Department of Psychiatry and Psychotherapy, University Medical Center Göttingen; Department of Neuroscience, University of Pittsburgh, USA

\section{Members of the Examination Board}

\section{Reviewer: Prof. Dr. Dr. Oliver Schlüter}

Department of Psychiatry and Psychotherapy, University Medical Center Göttingen;

Department of Neuroscience, University of Pittsburgh, USA

\section{$2^{\text {nd }}$ Reviewer: Prof. Dr. Siegrid Löwel}

Department of Systems Neuroscience, Johann-Friedrich-Blumenbach-Institute of Zoology and Anthropology, University of Göttingen

\section{Further Members of the Examination Board}

Prof. Dr. Michael Müller

Institute of Neuro- and Sensory Physiology, University Medical Center Göttingen

\section{Prof. Dr. Dr. Hannelore Ehrenreich}

Department of Clinical Neuroscience, Max-Planck-Institute of Experimental Medicine

\section{Prof. Dr. Oliver Wirths}

Department of Psychiatry and Psychotherapy, University Medical Center Göttingen

\section{Prof. Dr. Ralf Heinrich}

Department of Cellular Neurobiology, Schwann-Schleiden Research Center, University of Göttingen

Date of the oral examination: Dec 18, 2018 


\section{Affidavit}

I hereby declare that the presented thesis entitled "The role of DLG-MAGUKs in mediating signaling specificity at the postsynaptic density" has been written independently and with no other sources and aids than quoted.

Joana-Kristin Duda

Göttingen, November 2018 
If the human brain were so simple that we could understand it, we would be so simple that we couldn't.

- Emerson M. Pugh 



\section{TABLE OF CONTENTS}

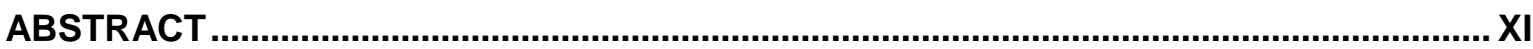

LIST OF FIGURES.......................................................................................................... XIII

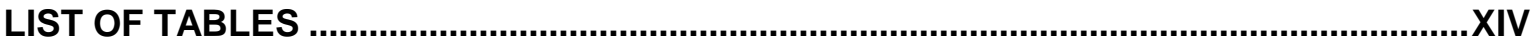

LIST OF ABBREVIATIONS...........................................................................................XV

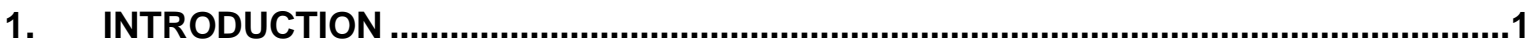

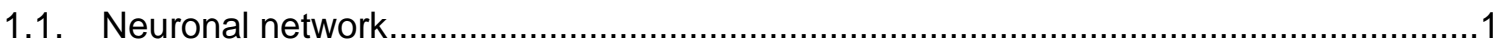

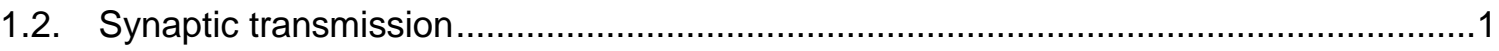

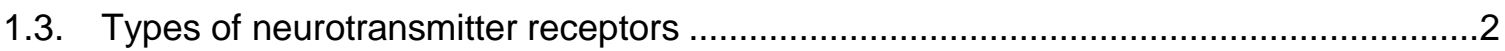

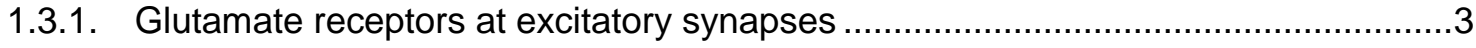

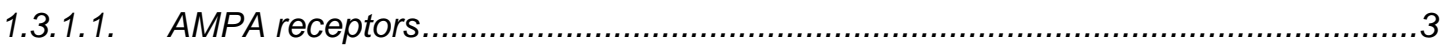

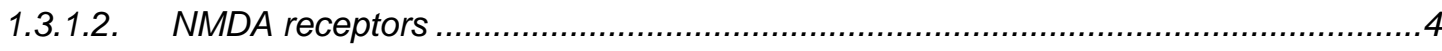

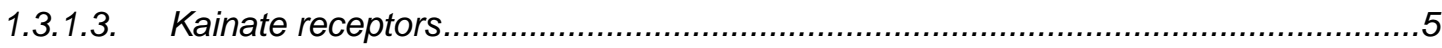

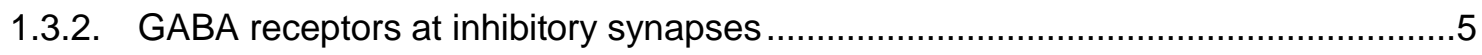

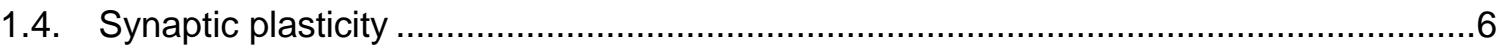

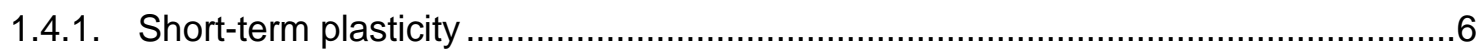

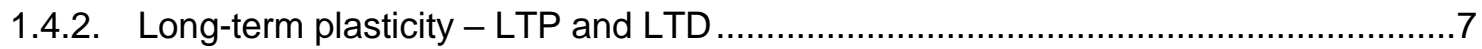

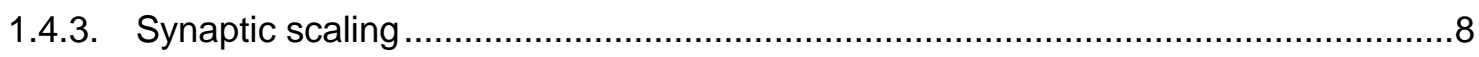

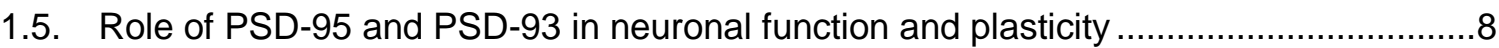

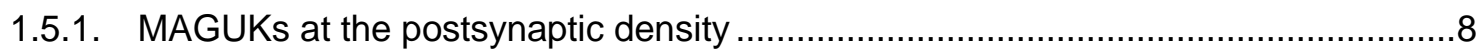

1.5.2. Developmental profiles und functions of DLG-MAGUKs......................................

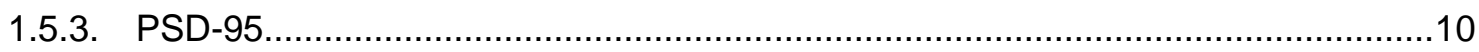

1.5.4. PSD-93

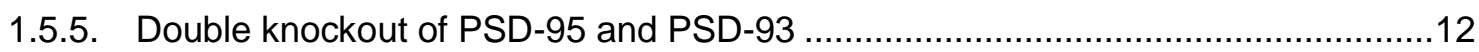

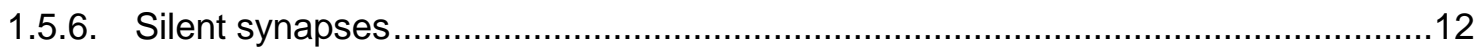

1.6. Link of glutamate receptor complexes to neuronal dysfunction ..................................15

1.6.1. Genetic studies link PSD-95 and PSD-93 to neuro-developmental and neuropsychiatric disorders 
1.6.2. Involvement of PSD-95 and PSD-93 in NMDAR-mediated excitotoxicity ..............16

1.6.2.1. Extrasynaptic NMDAR/calpain-mediated excitotoxicity ..................................... 16

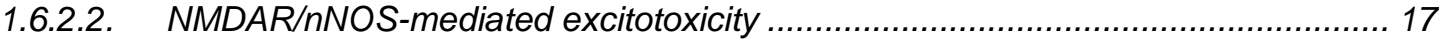

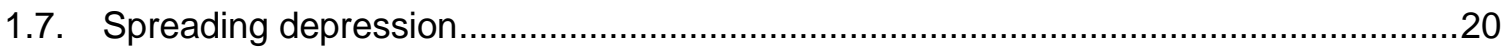

1.7.1. Characteristics and clinical relevance of spreading depression ...........................20

1.7.2. Spreading depression-associated intrinsic optical signal.....................................22

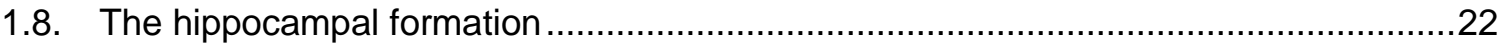

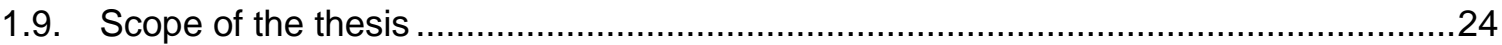

1.9.1. Can the absence of PSD-95 and/or PSD-93 provide protection against hypoxic insults?

1.9.2. How does the synaptic composition change upon KO of PSD-95 and/or PSD$93 ? 25$

2. MATERIALS AND METHODS

2.1. Materials 27

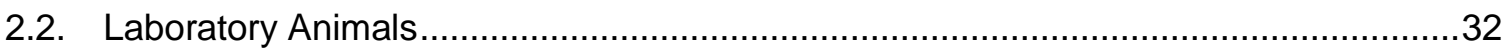

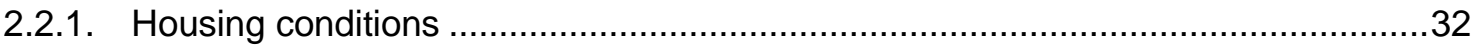

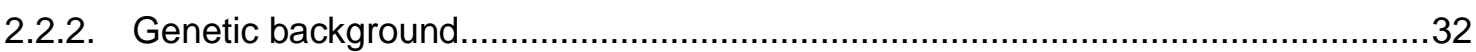

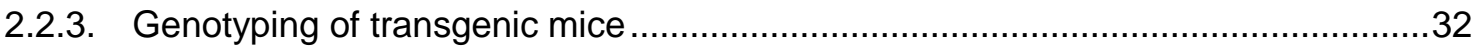

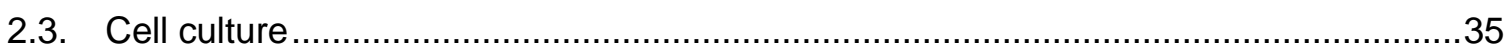

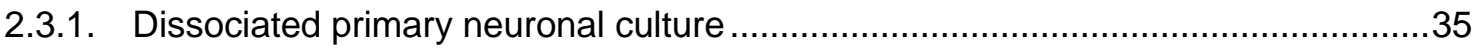

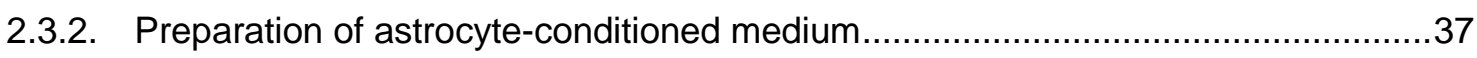

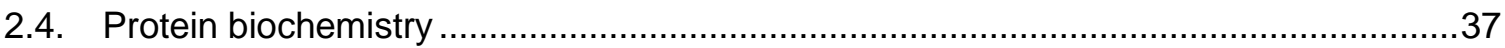

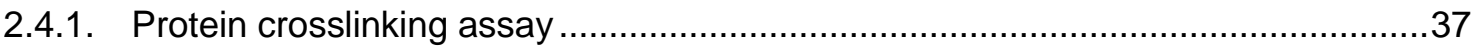

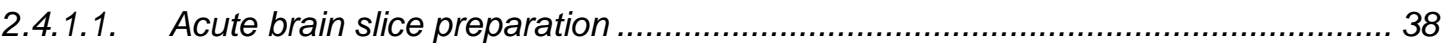

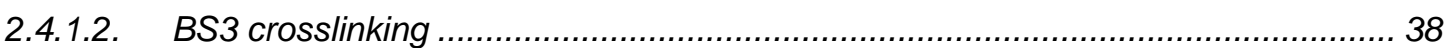

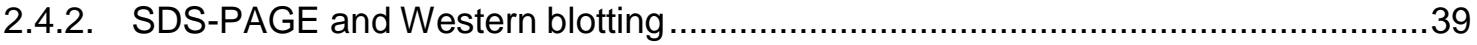

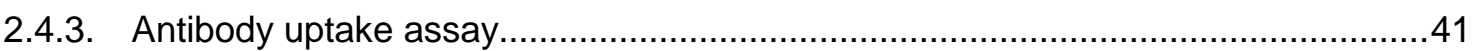

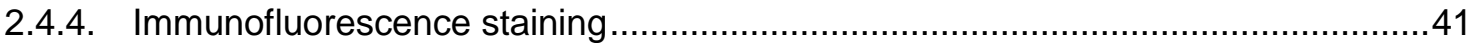

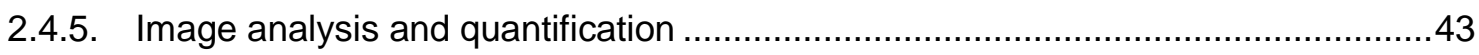

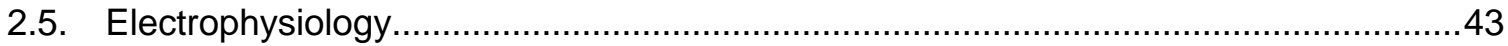


2.5.1. Acute brain slice preparation for electrophysiology ........................................... 43

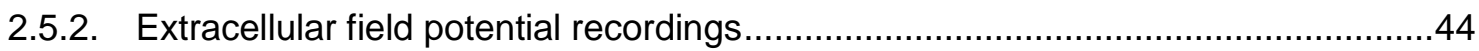

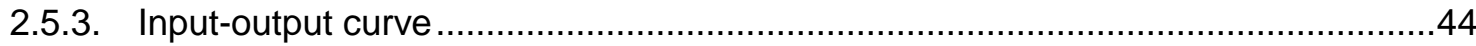

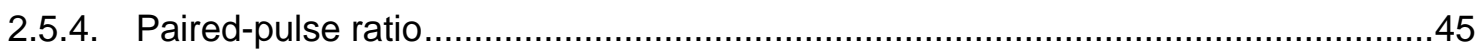

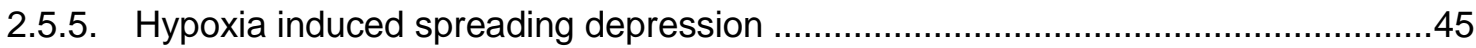

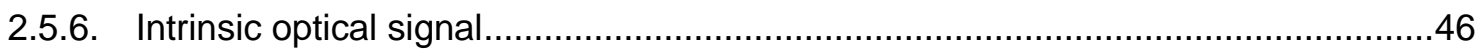

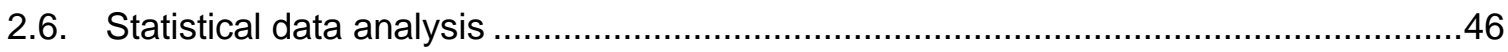

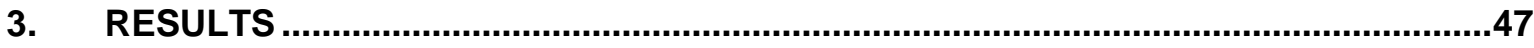

3.1. Protective effect of PSD-95 KO against hypoxia-induced synaptic dysfunction ...........47

3.1.1. Altered synaptic transmission and short-term plasticity in MAGUK KO mice .........48

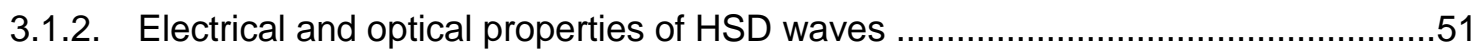

3.1.3. Delayed onset of hypoxia-induced spreading depression in PSD-95 KO mice ......54

3.1.4. Modified intrinsic optical signals indicate a protective capacity of PSD-95 loss

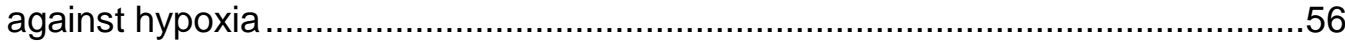

3.1.4.1. Loss of PSD-95 attenuates the increase of tissue light reflectance during HSD ...56

3.1.4.2. Trend to decelerated HSD wave propagation in PSD-95 KO slices ......................59

3.1.5. Partly reduced hypoxia-induced synaptic dysfunction in PSD-95 KO and PSD93/95 DKO mice 60

3.1.5.1. Reduced excitability of $P S D-95 K O$ and $P S D-93 / 95$ DKO neuronal networks.......60

3.1.5.2. Loss of synaptic function in acute brain slices due to oxygen deprivation .............62

3.1.5.3. $P S D-95 K O$ and $P S D-93 / 95$ DKO mice display improved posthypoxic recovery...64

3.2. Synaptic composition of excitatory cortical neurons in MAGUK KO mice 67

3.2.1. Similar cortical protein levels of glutamate and GABA receptors in WT and PSD-95 KO mice

3.2.2. Slight effects on GluR surface expression due to the absence of PSD-95............72

3.2.3. Investigation of the TCM effect on AMPAR-mEPSC frequency .............................77

3.2.3.1. Synaptotagmin 1 co-localizes with the presynaptic markers Munc13, VGLUT1 and Ankyrin-B .78

3.2.3.2. The TCM-evoked increase in AMPAR-mEPSC frequency is not a presynaptic effect 
4. DISCUSSION

4.1. Knockout of PSD-95 leads to reduced excitatory synaptic strength ............................83

4.2. Partly altered synaptic composition in MAGUK KO mice............................................ 86

4.2.1. Evidence for extrasynaptic AMPARs in silent synapses ......................................86

4.2.2. Impaired GluN2B to GluN2A switch in PSD-95 KO mice.....................................88

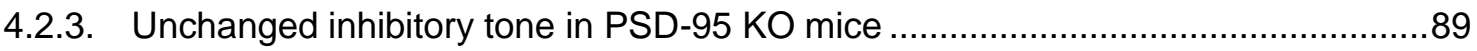

4.2.4. Homeostasis of spontaneous synaptic transmission is primarily a postsynaptic

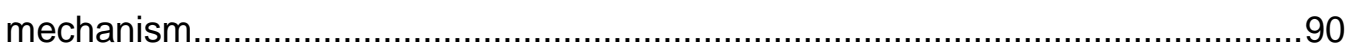

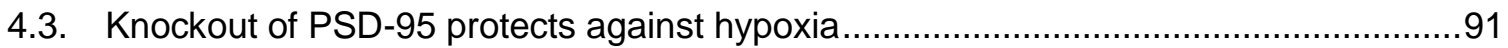

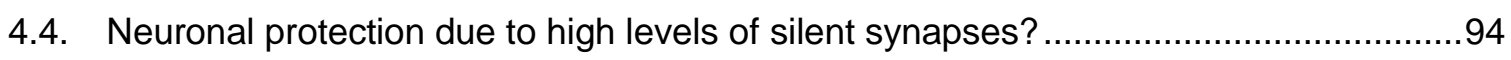

4.4.1. CaMKII as a possible target to inhibit silent synapse maturation ..........................95

4.5. Neuronal protection due to attenuated NMDAR-mediated excitotoxicity?.....................96

4.5.1. Reduced activity of the NMDAR-nNOS pathway in PSD-95 KO mice? ..................97

4.6. How do PSD-95 and PSD-93 mediate different signaling cascades? .........................99

4.6.1. PSD-95 and PSD-93 might be localized at different neuronal sites ......................99

4.6.2. Potential interaction partners of PSD-95 and PSD-93 …..............................100

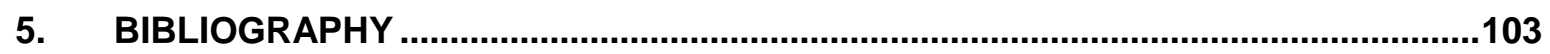

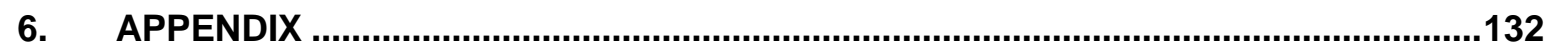

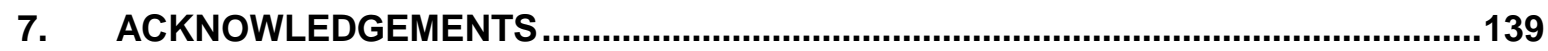




\section{ABSTRACT}

Ischemic stroke is one of the leading causes of long-term disability and death worldwide. Despite the importance of medical treatment, the underlying molecular mechanisms are not fully understood. A critical factor of ischemic stroke is hypoxia, leading to hypoxia-induced spreading depression (HSD) in the affected brain tissue. HSD is characterized by strong cell depolarization and disrupted ion homeostasis, which shuts down neuronal function and can cause severe cell damage. Importantly, two DLG-MAGUKs (Discs large membrane-associated guanylate kinases), namely PSD-95 and PSD-93, have been implicated in NMDA receptor (NMDAR)-mediated excitotoxicity processes contributing to stroke pathology. Both proteins are important players in the core organization of the postsynaptic density and exhibit opposing roles in experience-dependent synapse maturation during early development and in synaptic plasticity in the adult brain. The present study aimed at further investigating the role of PSD-95 and PSD-93 in synaptic transmission and excitotoxicity processes using a model of transient hypoxia in acute hippocampal slices of adult PSD-95 knockout (KO), PSD-93 KO and PSD93/95 double knockout (DKO) mice.

The strength of basal synaptic transmission was substantially reduced in PSD-95 KO and PSD-93/95 DKO, but not in PSD-93 KO mice. Diminished synaptic function of PSD-95 KO mice is consistent with the previous finding that they maintain high levels of silent synapses into adulthood. Silent synapses lack functional AMPARs and therefore do not transmit at resting potential. Normally, such silent synapses undergo experience-dependent maturation into AMPAR-transmitting synapses during early development, thereby consolidating preexisting wiring patterns. In contrast to PSD-95 KO, silent synapses in PSD-93 KO mice mature faster during development than in wildtype (WT) mice. Despite silent synapse number in younger DKO mice has been reported to be unchanged, the present results strongly indicate impaired excitatory transmission in adult DKO mice.

Differential effects of MAGUK KO were further seen in HSD. Importantly, the absence of PSD95 provided protection against hypoxia-induced metabolic compromise of neuronal function. Indeed, PSD-95 KO mice exhibited reduced HSD effects as demonstrated by multiple parameters including delayed onset of HSD, attenuated change of intrinsic optical signals and improved recovery of synaptic function. By contrast, single KO of PSD-93 had no effect on the susceptibility to hypoxia. Interestingly, the protective effects in PSD-95 KO mice were consistently abolished by additional KO of PSD-93, except for improved synaptic recovery. Given its interaction with NMDARs, PSD-95 might couple the massive hypoxia-induced calcium influx to downstream signaling cascades involved in excitotoxicity processes such as toxic nitric oxide production. Toxic signal transduction mainly occurs at extrasynaptic sites involving GluN2B-containing NMDARs. Notably, further biochemical analysis of brain 
homogenates revealed an impaired switch from GluN2B- to GluN2A-NMDARs in PSD-95 KO mice, which normally occurs at synaptic sites during early development. Thus, silent synapses might mainly contain synaptic GluN2B-NMDARs, rather than extrasynaptic ones mediating excitotoxicity, therefore providing protection. Another important aspect of high silent synapse levels in PSD-95 KO mice is the increased potential for synaptic plasticity, maintaining the brain in a partly premature state. Since stroke is highly influenced by aging, which is associated with low silent synapse levels, increased levels of silent synapses may represent an important substrate for the reduced hypoxia-susceptibility in PSD-95 KO mice. Silent synapses provide opportunities of reorganization and refinement of neuronal networks and might therefore be particularly beneficial for synaptic recovery, which is consistent with the improved recovery in the absence of PSD-95.

Taken together, my data reveals that the KO of PSD-95 provides protection against hypoxia, which is likely due to high levels of silent synapses. 


\section{LIST OF FIGURES}

Figure 1: Link between NMDARs and nNOS-mediated neurotoxicity through PSD-95 …....19

Figure 2: The neuronal circuitry in the rodent hippocampus. ............................................2

Figure 3: Schematic illustration of fEPSP recordings in the hippocampus. ........................47

Figure 4: Changed hippocampal synaptic transmission and short-term synaptic plasticity

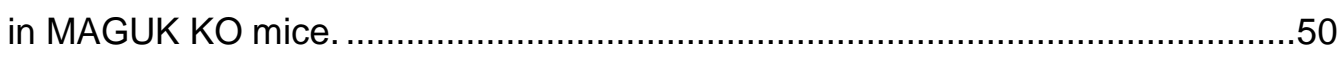

Figure 5: Intrinsic optical signal of the HSD wave in hippocampal slices.............................51

Figure 6: HSD-accompanied negative deflection of the DC potential. .................................53

Figure 7: Time to HSD onset was prolonged by loss of PSD-95 .........................................55

Figure 8: Reduced light reflectance response of hippocampal tissue in PSD-95 KO mice

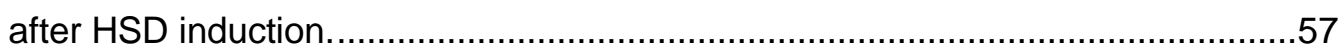

Figure 9: The HSD wave proliferated more slowly in the absence of PSD-95.....................60

Figure 10: PSD-95 KO and PSD-93/95 DKO fEPSP amplitudes showed 50\% reduction. .....61

Figure 11: Hypoxia-induced loss of synaptic function in acute hippocampal slices................63

Figure 12: Brain slices from PSD-95 KO and PSD-93/95 DKO mice demonstrated earlier and increased posthypoxic recovery.

Figure 13: Developmental profile of cortical protein expression in WT and PSD-95 KO brain slices.

Figure 14: Comparison of glutamate and $\mathrm{GABA}_{A}$ receptor levels in WT and PSD-95 KO cortices.

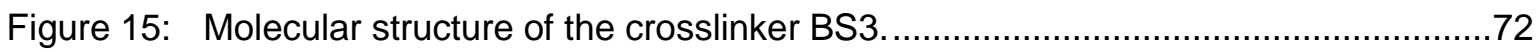

Figure 16: Surface levels of AMPAR and NMDAR subunits in WT and MAGUK KO mice at P28-32.

Figure 17: Developmental profile of AMPAR, NMDAR and $G_{A B A}$ receptor surface expression in WT and PSD-95 KO cortices.

Figure 18: Molecular mechanism of the Synaptotagmin 1 uptake assay for monitoring presynaptic activity.

Figure 19: Immunofluorescence staining showed co-localization of Synaptotagmin 1 with diverse presynaptic markers.

Figure 20: Synaptotagmin 1 uptake assay did not reveal an effect of TCM on presynaptic activity.

Figure 21: Developmental profile of AMPAR-mEPSC frequency and effect of TCM. 133 


\section{LIST OF TABLES}

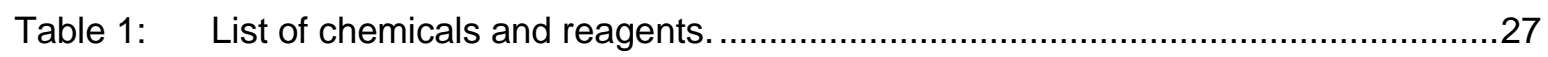

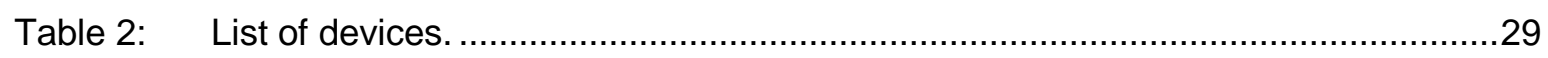

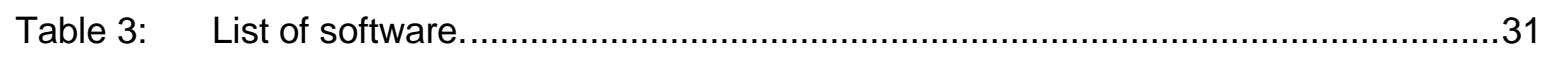

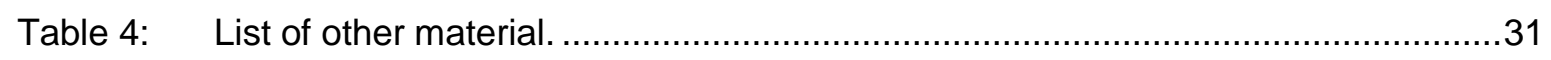

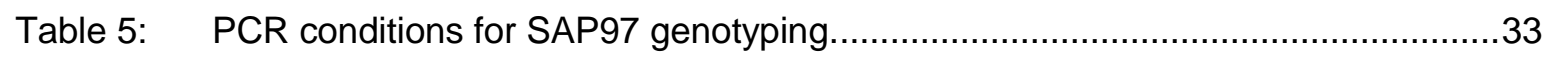

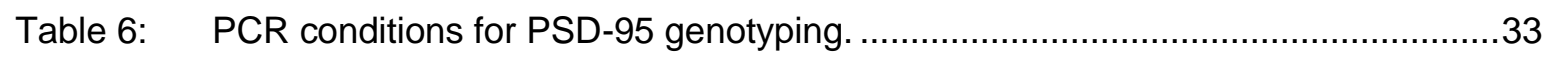

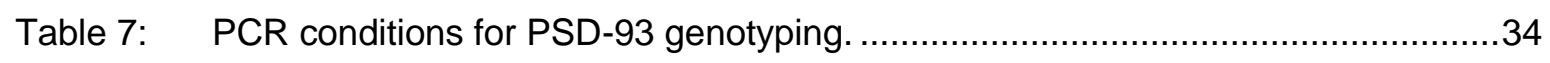

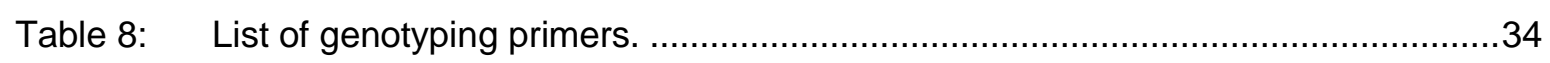

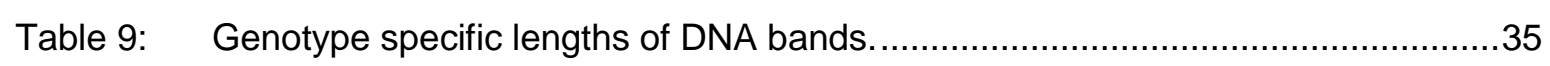

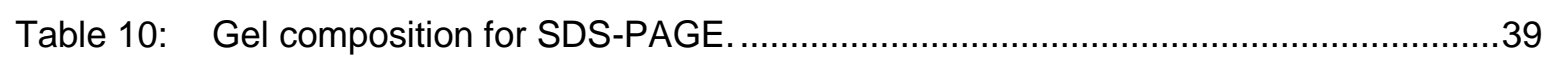

Table 11: Antibodies used for immunoblotting. ............................................................. 40

Table 12: Antibodies used for immunofluorescence staining ............................................42

Table 13: Developmental profile of glutamatergic and GABAergic receptor subunits in WT

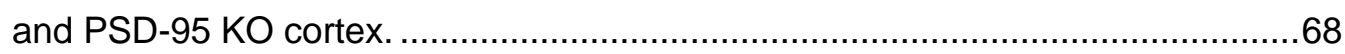

Table 14: Cortical surface levels of AMPAR and NMDAR subunits in WT and MAGUK

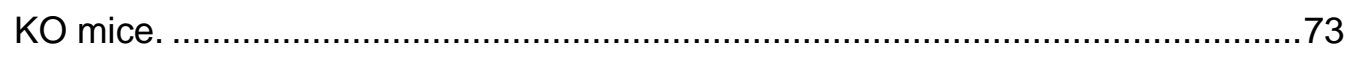

Table 15: fEPSP slope/FV amplitude ratios of I-O curves. .............................................132

Table 16: EPSP2/EPSP1 amplitude ratios of paired-pulse facilitation..............................132

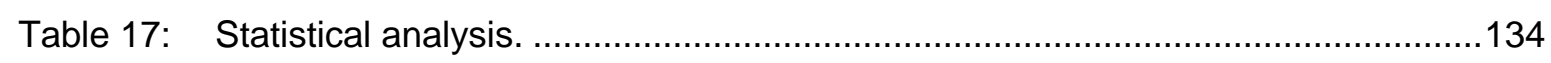




\section{LIST OF ABBREVIATIONS}

ACSF

ADP

AMBA

AMPA

AMPAR

ANOVA

APS

ASD

ATP

BS3

BSA

$\mathrm{CA}$

cAMP

CNS

C-terminal

DC

DG

DIV

DKO

DLG-MAGUKs

DMEM

DNA

dNTP

DTT

EDTA

fEPSP

FBS

FV

g
Artificial cerebrospinal fluid

Adenosine diphosphate

Acrylamide/bis-acrylamide solution

a-Amino-3-hydroxy-5-methyl-4-isoxazolepropionic

a-Amino-3-hydroxy-5-methyl-4-isoxazolepropionic acid receptor

Analysis of variance

Ammonium peroxodisulfate

Autism spectrum disorder

Adenosine 5'-triphosphate

Bis(sulfosuccinimidyl)suberate, crosslinker

Bovine serum albumin

Cornu Ammonis

3',5'-Cyclic adenosine monophosphate

Central nervous system

Carboxy-terminal

Direct current

Dentate gyrus

Days in vitro

Double knockout

Discs large (DLG) protein family of membrane-associated guanylate kinases (MAGUKs)

Dulbecco's Modified Eagle Medium

Deoxyribonucleic acid

Deoxyribonucleotide triphosphate

Dithiothreitol

Ethylenediaminetetraacetic acid

Field excitatory postsynaptic potential

Fetal bovine serum

Fiber volley

Ground acceleration 


\begin{tabular}{|c|c|}
\hline GABA & Y-Aminobutyric acid \\
\hline GAPDH & Glyceraldehyde-3-phosphate dehydrogenase \\
\hline GluR & Glutamate receptor \\
\hline GTP & Guanosine 5'-triphosphate \\
\hline HEPES & 4-(2-Hydroxyethyl)-1-piperazineethanesulfonic acid \\
\hline HSD & Hypoxia-induced spreading depression \\
\hline $\lg G$ & Immunoglobulin G \\
\hline iGluR & lonotropic glutamate receptor \\
\hline I-O curve & Input-output curve \\
\hline IOS & Intrinsic optical signal \\
\hline IPSP & Inhibitory postsynaptic potential \\
\hline KAR & Kainate receptor \\
\hline $\mathrm{kb}$ & Kilobase \\
\hline KD & Knockdown \\
\hline $\mathrm{kDa}$ & Kilodalton \\
\hline KO & Knockout \\
\hline LSD & Least significant difference \\
\hline LTD & Long-term depression \\
\hline LTP & Long-term potentiation \\
\hline MAGUK & Membrane-associated guanylate kinase \\
\hline MAP2 & Microtubule-associated protein 2 \\
\hline MAPK & Mitogen-activated protein kinase \\
\hline MCAO & Middle cerebral artery occlusion \\
\hline mEPSC & Miniature excitatory postsynaptic current \\
\hline mGluR & Metabotropic glutamate receptor \\
\hline munc & Mammalian unc (uncoordinated) homolog \\
\hline MW & Molecular weight \\
\hline NEB & New England Biolabs GmbH \\
\hline NMDA & N-methyl-D-aspartate \\
\hline NMDAR & $\mathrm{N}$-methyl-D-aspartate receptor \\
\hline nNOS & Neuronal nitric oxide synthase \\
\hline NO & Nitric oxide \\
\hline
\end{tabular}




\begin{tabular}{|c|c|}
\hline n.s. & Not significant \\
\hline NT & Neurotransmitter \\
\hline $\mathrm{N}$-terminal & $\mathrm{NH}_{2} /$ amino-terminal \\
\hline OD & Ocular dominance \\
\hline $\mathrm{P} / \mathrm{PD}$ & Postnatal day \\
\hline PAF & Platelet-activating factor \\
\hline PAGE & Polyacrylamide gel electrophoresis \\
\hline PARP & Poly (ADP-ribose) polymerase \\
\hline PBS & Phosphate buffered saline \\
\hline PCR & Polymerase chain reaction \\
\hline PDZ & PSD-95, discs-large tumor suppressor, zonula occludens-1 protein \\
\hline PFA & Paraformaldehyde \\
\hline $\mathrm{pH}$ & Negative decadic logarithm of the hydrogen ion concentration \\
\hline $\mathrm{PKC} \beta$ & Protein kinase $C$ beta type \\
\hline Pol & Polymerase \\
\hline PPR & Paired-pulse ratio \\
\hline $\operatorname{Pr}$ & Release probability \\
\hline PSD & Postsynaptic density \\
\hline $\mathrm{PV}+$ & Parvalbumin-positive \\
\hline RNA & Ribonucleic acid \\
\hline RNAi & RNA interference, e.g. short-hairpin RNA (shRNA) \\
\hline $\mathrm{RE}$ & Recording electrode \\
\hline $\mathrm{ROI}$ & Region of interest \\
\hline ROS & Reactive oxygen species \\
\hline rpm & Revolutions per minute \\
\hline $\mathrm{RT}$ & Room temperature \\
\hline SAP & Synapse-associated protein \\
\hline SAPAP & SAP90/PSD-95-associated protein \\
\hline SC & Schaffer collateral \\
\hline SD & Spreading depression \\
\hline SDS & Sodium dodecyl sulfate \\
\hline SE & Stimulation electrode \\
\hline
\end{tabular}


SEM Standard error of the mean

$\mathrm{SH} 3$

Src homology 3

SHP-2 Src homology 2 domain-containing phosphatase

st.

Stratum

SynGAP

Synaptic GTPase activating protein

Syt1

Synaptotagmin 1

TARP

Transmembrane AMPA receptor regulatory protein

TCM

Trichlormethiazide

TEMED

Tetramethylethylenediamine

V1

Primary visual cortex

VGLUT1

Vesicular glutamate transporter 1

WB

Western blot

$\mathrm{w} / \mathrm{o}$

Without

WT

Wildtype 


\section{INTRODUCTION}

\subsection{Neuronal network}

The central nervous system (CNS) mediates all our thoughts, emotion and behavior. As diverse the neuronal network is, so various are impairments, dysfunctions and diseases occurring on the neuronal level, which we are trying to understand and in the best case, prevent or cure. To enable appropriate research aiming in improving medical development, we have to investigate and understand the molecular processes underlying neuronal function. These processes occur in the neurons themselves, in their interaction with surrounding glial cells and in specialized structures called synapses. Synapses are the pivotal spots where two neurons come close enough to each other so that they are able to communicate. In a mature network composed of various interconnected neurons, information is transferred via electrical signals passing through the presynaptic neuron towards the synaptic cleft, where information is transmitted to the postsynapse. In the case of excitatory synapses, the postsynaptic density (PSD), which contains neurotransmitter receptors and an elaborate complex of other proteins, is receiving the signals in order to transfer and react to incoming information. This study addresses specific PSD-located proteins which are thought to execute a crucial role in synaptic plasticity - the basis of learning and memory - and have further been implicated in mechanisms of neuronal disorders.

\subsection{Synaptic transmission}

Every neuron generates around 1000 synaptic connections to neighboring cells and receives about 10,000 or even more. In this huge network, information is propagated via action potentials. In general, synapses can be electrical or chemical. Electrical synapses directly transfer signals as ion currents across small gap junctions of $\sim 3.5 \mathrm{~nm}$ width. In contrast, the presynaptic neuron of chemical synapses first converts electrical signals into neurotransmitter. These transmitters are released into the $20-40 \mathrm{~nm}$ wide synaptic cleft to mediate either excitatory or inhibitory actions in the postsynaptic cell. Specifically, when an action potential reaches the presynaptic axon terminal, voltage-gated calcium $\left(\mathrm{Ca}^{2+}\right)$ channels open, resulting in high $\mathrm{Ca}^{2+}$ influx into the so-called "active zone". Subsequently, specialized vesicles fuse with the presynaptic membrane and release neurotransmitters into the synaptic cleft. These chemical messengers can diffuse through the cleft and bind to receptors on the postsynaptic membrane, causing ion channels to open and therefore modifying conductance and potential of the postsynaptic cell. Thereby, the chemical signal is re-converted into an electrical signal, 
which induces an action potential in the postsynaptic neuron, if a distinct threshold is reached (Kandel et al., 2000).

\subsection{Types of neurotransmitter receptors}

Neurotransmitters can act via binding to either ionotropic or metabotropic receptors. Ionotropic receptors are also termed ligand-gated channels. These macromolecules are integral membrane proteins containing an extracellular domain forming the neurotransmitter binding pocket and a transmembrane domain which constitutes the ion channel. The channel opens upon conformational change caused by neurotransmitter binding. On the contrary, metabotropic receptors, such as serotonin or norepinephrine receptors in the cerebral cortex, are located distinctly from the ion channels they act on and therefore require another signaling machinery to transfer the incoming information. Specifically, the so-called G-protein-coupled receptors activate guanosine triphosphate (GTP)-binding proteins which often engage in a second messenger cascade triggering the production of cyclic adenosine monophosphate (cAMP). In case of receptor tyrosine kinases, the incoming signal is transferred via a protein phosphorylation cascade (Kandel et al., 2000).

Depending on the ion channel type gated by the incoming neurotransmitter, the effect of a chemical synapse can be modulatory, inhibitory or excitatory. Modulatory transmitters, such as serotonin and dopamine, act via second messengers in order to modify the metabolism or membrane permeability of the postsynaptic neuron. In contrast, the neurotransmitters $Y$ aminobutyric acid (GABA) and glycine typically affect inhibitory ionotropic receptors. Glutamate - the major excitatory transmitter in the CNS - primarily acts on receptors that produce excitation.

Glutamate receptors (GluRs) conduct cations, mainly sodium $\left(\mathrm{Na}^{+}\right)$and potassium ions $\left(\mathrm{K}^{+}\right)$, thereby provoking depolarization of the postsynaptic cell. Specifically, the activated presynapse releases glutamate with a certain probability ( $\mathrm{Pr}$, release probability) into the synaptic cleft. Subsequent binding of glutamate to postsynaptic GluRs leads to $\mathrm{Na}^{+}$influx which in turn generates a depolarizing excitatory postsynaptic current (EPSC). If the membrane potential reaches a certain threshold, an action potential is evoked at the axon hillock, which travels along the axon and reaches the presynaptic terminals, where it again triggers neurotransmitter release. On the contrary, inhibitory neurotransmitters mainly affect channels permeable to chloride ions $\left(\mathrm{Cl}^{-}\right)$, thereby hyperpolarizing the postsynaptic membrane which leads to reduced probability of action potential firing.

Regarding one single neuron, the convergence of many excitatory synaptic potentials from afferent neurons need to be integrated to reach the threshold for an action potential. This can be countervailed by inhibitory inputs, preventing the membrane potential from reaching threshold (Kandel et al., 2000). 


\subsubsection{Glutamate receptors at excitatory synapses}

Glutamate is the major excitatory neurotransmitter in the brain and can activate both metabotropic and ionotropic receptors. Metabotropic GluRs (mGluRs) are G-protein coupled receptors mediating relatively slow or modulatory responses to glutamate through intracellular signaling of second messenger cascades (Conn \& Pin, 1997). On the other hand, ionotropic GluRs (iGluRs) - which will play an important role in this study - mediate rather fast synaptic transmission. They consist of three major groups, i.e. AMPA, NMDA and kainate receptors, named according to the synthetic agonist that preferentially stimulates them (Nicoll et al., 1990).

\subsubsection{AMPA receptors}

a-Amino-3-hydroxy-5-methyl-4-isoxazolepropionic receptors (AMPARs) are the predominant fast signaling element at synapses and play a key role in the expression of plasticity (Traynelis et al., 2010). The rapid kinetics of AMPARs enable fast depolarization of the postsynaptic neuron and thus high-fidelity transfer of information. During synaptic plasticity, AMPARs can change in their number by lateral diffusion, endocytosis or exocytosis; interact with different binding partners or vary in their phosphorylation state and subunit composition (Malinow \& Malenka, 2002; Bredt \& Nicoll, 2003; Shepherd \& Huganir, 2007).

Functional AMPARs are homo- or heterotetramers composed of four core subunits GluA1-A4, with each of them having distinct influence on AMPAR trafficking properties, channel kinetics and ion selectivity (Wisden \& Seeburg, 1993; Hollmann \& Heinemann, 1994). Thus, heteromerization produces high diversity in AMPAR function.

The most prominent example is the impact exerted by presence or absence of the subunit GluA2 in the receptor signaling machinery. The majority of AMPARs are GluA2-containing heteromers which are impermeable to $\mathrm{Ca}^{2+}$. In contrast, GluA2-lacking AMPARs also conduct $\mathrm{Ca}^{2+}$ and are therefore supposed to play important roles in the activation of downstream signaling cascades, eventually affecting postsynaptic mechanisms like synaptic plasticity and disease-related neuronal changes (Dingledine et al., 1999; Burnashev \& Rozov, 2000; CullCandy et al., 2006; Lüscher \& Malenka, 2011).

The subunit composition varies among brain regions and developmental stages. Whereas GluA4-containing AMPARs are found in immature hippocampal neurons, they are getting progressively replaced by GluA2-containing AMPARs during development (Zhu et al., 2000). In mature hippocampus and cortex, the predominant AMPARs are GluA1/A2 and to a lesser extent GluA2/A3 heterodimers (Wenthold et al., 1996; Lu et al., 2009). While each subunit possesses one binding site for the neurotransmitter glutamate, the channel only opens if two 
glutamate molecules are bound to the receptor complex, resulting in cation flow in respect to their electrochemical gradient (Clements et al., 1997; Rosenmund et al., 1998).

\subsubsection{NMDA receptors}

Similar to AMPARs, N-methyl-D-aspartate receptors (NMDARs) are tetrameric receptor complexes. They are assembled out of seven subunits, namely GluN1, GluN2A-D, GluN3A and GluN3B (also referred to as NR1, NR2A-D, NR3A and NR3B), which form di- or triheteromers. Each receptor consists of two obligatory GluN1 subunits with either two GluN2 or a mixture of GluN2 with GluN3 subunits (Monyer et al., 1992; Schorge \& Colquhoun, 2003; Ulbrich \& Isacoff, 2007). The immense diversity of subunit composition generates NMDARs with distinct pharmacological properties and biological functions (Vicini et al., 1998). They are involved in various neuronal processes, such as synaptic potentiation and depression, cell survival and trauma resistance, but also in neuronal cell death (Cull-Candy \& Leszkiewicz, 2004; Papadia \& Hardingham, 2007).

NMDARs require two co-agonists for activation and certain electrophysiological circumstances. While glutamate binds to GluN2 subunits, GluN1 contains a binding site for glycine (Benveniste \& Mayer, 1991; Clements \& Westbrook, 1991). Moreover, NMDARs are coupled to voltage-sensitive conductance: at resting membrane potential (-60 to $-70 \mathrm{mV}$ ) the cation channel is blocked by an extracellular magnesium ion $\left(\mathrm{Mg}^{2+}\right)$, which will only be released upon membrane depolarization (Mayer et al., 1984; Nowak et al., 1984). Opening of the pore primarily allows influx of $\mathrm{Ca}^{2+}$, but as well influx of $\mathrm{Na}^{+}$and efflux of $\mathrm{K}^{+}$ions, according to their respective electrochemical gradient. $\mathrm{Ca}^{2+}$ influx into the postsynapse can affect diverse downstream signaling cascades, e.g., mediating synaptic plasticity or cell fate (Bliss \& Collingridge, 1993; Kessels \& Malinow, 2009).

NMDARs are incorporated into glutamatergic synapses early in development. However, their subunit composition changes during activity- and experience-dependent critical periods, which is accompanied by alterations in receptor kinetics and synaptic localization (Hadzic et al., 2017). At birth, the predominant subunit in the forebrain - apart from obligatory GluN1 - is GluN2B, while more GluN2A subunits are progressively incorporated during development, triggered by receptor activity and sensory input (Monyer et al., 1994; Sheng et al., 1994). NMDARs in the adult hippocampus and neocortex are mostly composed of GluN1/2A and to a lesser extent of GluN1/2B heteromers, having relatively high ion conductance and sensitivity to the $\mathrm{Mg}^{2+}$ block (Monyer et al., 1994; Sheng et al., 1994). The subunit GluN2C appears postnatally in the cerebellum, whereas GluN2D is found in the brain stem and diencephalon during embryonic and neonatal stages (Monyer et al., 1994; Goebel \& Poosch, 1999). GluN3 subunits are mainly present at late prenatal and early postnatal brain development (Sun et al., 
1998). All three of them (GluN2C, GluN2D and GluN3) render the NMDAR complex less sensitive to $\mathrm{Mg}^{2+}$ blockage and less conductive (Pérez-Otaño \& Ehlers, 2004; Pachernegg et al., 2012).

\subsubsection{Kainate receptors}

Kainate receptors (KARs) mediate synaptic transmission both pre- and postsynaptically and are ubiquitously expressed in the CNS (Petralia et al., 1994; Frerking \& Nicoll, 2000; Gallyas et al., 2003). At the postsynapse, KARs modulate the membrane potential similar to NMDARs and AMPARs, whereas at the presynaptic level, they influence neurotransmitter release both at excitatory and inhibitory synapses (Kullmann, 2001; Lauri et al., 2001). KARs share several agonists and antagonists with AMPARs, therefore both receptors are collectively referred to as "non-NMDA receptors". The issue of common binding partners hindered investigations on the physiological role of KARs for many years, lacking pharmacological tools selective for this receptor type (Lerma, 2003).

It could be shown that, like the other iGluRs, KARs are formed by tetrameric assembly of receptor subunits around a central ion pore. They can build homo- or heteromers out of subunits GluR5-7, which eventually co-assemble with KA-1 and KA-2 subunits (Gallyas et al., 2003; Lerma, 2003).

KARs are implicated in synaptic plasticity (Bortolotto et al., 1999; Contractor et al., 2001) and furthermore linked to the generation of seizure activity (Mulle et al., 1998).

\subsubsection{GABA receptors at inhibitory synapses}

GABA is the major inhibitory neurotransmitter in the adult brain. It mainly acts through ionotropic GABA type $A\left(G A B A_{A}\right)$ receptors and to a lesser extent via metabotropic GABA type $B\left(G A B A_{B}\right)$ receptors. Together with the main excitatory neurotransmitter glutamate, GABA regulates the balance of neuronal excitation and inhibition, which is crucial for proper brain function (Wu \& Sun, 2015).

In the adult mammalian CNS, GABA predominantly interacts with postsynaptically located $G_{A B A}$ receptors. Upon GABA release from presynaptic vesicles and its binding to the receptor, the ion channel opens and mediates transient $\mathrm{Cl}^{-}$influx along the electrochemical gradient. This evokes a hyperpolarizing postsynaptic response - the inhibitory postsynaptic potential (IPSP) - which lowers the likelihood of action potential firing in the postsynaptic cell (Farrant \& Nusser, 2005). Apart from this fast mechanism, also referred to as "phasic inhibition", GABA can as well bind to high-affinity extrasynaptic receptors, resulting in a so- 
called persistent or "tonic" inhibition (Kaneda et al., 1995; Bai et al., 2001; Semyanov et al., 2003; Farrant \& Nusser, 2005).

$\mathrm{GABA}_{\mathrm{A}}$ receptors are anion-specific channels and comprise a heterotetrameric structure. In the human brain, various subunits have been discovered, i.e., $6 \alpha$ subunits, $3 \beta$ subunits, $3 y$ subunits, $3 \rho$ subunits, and one $\varepsilon, \delta, \phi$ and $\pi$ subunit, respectively. GABA $A_{A}$ receptors with unique subunit compositions are differentially expressed throughout the mature brain (Macdonald \& Olsen, 1994; Blednov et al., 2014). While receptors which contain the subunits $\alpha 1$ and $\mathrm{\gamma} 2$ are localized at the postsynapse, the subunits $\alpha 4-6$ and $\delta$ were found peri- or extrasynaptically (Lee \& Maguire, 2014; Wu \& Sun, 2015).

Interestingly, apart from their inhibitory function, $\mathrm{GABA}_{\mathrm{A}}$ receptors are implicated in excitatory synaptic transmission during early development, playing a fundamental role in early maturation of diverse brain structures (Sernagor et al., 2010; Wang \& Kriegstein, 2011; BenAri et al., 2012). The depolarizing effect of $\mathrm{GABA}_{\mathrm{A}}$ receptors lies in the fact that immature neurons tend to accumulate $\mathrm{Cl}^{-}$in their intracellular space (via $\mathrm{Na}^{+}-\mathrm{K}^{+}-2 \mathrm{Cl}^{-}$co-transporter). Hence, $\mathrm{Cl}^{-}$diffuses out of the postsynaptic cell upon $\mathrm{GABA}_{\mathrm{A}}$ receptor activation, resulting in membrane depolarization. In later developmental stages, neurons predominantly express a different co-transporter $\left(\mathrm{K}^{+}-2 \mathrm{Cl}^{-}\right)$, thereby reducing intracellular $\mathrm{Cl}^{-}$levels and shift $\mathrm{GABA}_{\mathrm{A}}$ function from mainly excitatory to inhibitory and hyperpolarizing in adolescent and adult brains (Ben-Ari et al., 2012; Deidda et al., 2015).

\subsection{Synaptic plasticity}

Synaptic plasticity is the ability of synapses to strengthen or weaken in response to changes in activity, thereby rendering them bidirectionally plastic. The mechanisms involved are diverse and can be located presynaptically, e.g. by alterations in neurotransmitter release, or postsynaptically by affecting the strength of the postsynaptic response. Synaptic plasticity is assumed to be the key mechanism for multiple brain functions like learning, memory formation and consolidation, as well as developmental network refinement (Katz \& Shatz, 1996; Chater \& Goda, 2014).

\subsubsection{Short-term plasticity}

Short-term forms of synaptic plasticity are generally mediated by lateral diffusion of AMPARs between synaptic and peri-synaptic regions due to high receptor mobility - with a diffusion speed depending on neuronal activity (Borgdorff \& Choquet, 2002; Groc et al., 2004). Since AMPARs contain relatively low affinity for glutamate, they can only be effectively activated when located close or directly opposite to presynaptic sites of neurotransmitter release 
(Lisman \& Raghavachari, 2006). AMPAR surface mobility and their subsequent incorporation and retention at synaptic sites is affected by extracellular matrix components (Frischknecht et al., 2009; Szepesi et al., 2014), receptor endocytosis and recycling (Petrini et al., 2009).

\subsubsection{Long-term plasticity - LTP and LTD}

Long-term strengthening of synapses is referred to as long-term potentiation (LTP) and was first described by Bliss \& Lomo in 1973. LTP can be viewed as a long-lasting increase of synaptic transmission in an activity-dependent manner and is considered to be the cellular correlate of learning and memory. The pivotal change during LTP is an increase in AMPAR number at the postsynapse. This can be induced by high frequency stimulation leading to AMPAR opening, $\mathrm{Na}^{+}$influx and subsequent membrane depolarization, which activates NMDARs to allow $\mathrm{Ca}^{2+}$ influx. $\mathrm{Ca}^{2+}-$ as a second messenger - triggers downstream signaling cascades of phosphorylation events to potentiate synaptic transmission, involving transient activation of $\mathrm{Ca}^{2+}$ /calmodulin-dependent protein kinase II (CaMKII) upon autophosphorylation (Barria et al., 1997; Mammen et al., 1997; Shen \& Meyer, 1999; Lee et al., 2009).

There is a distinct proportion of synapses which just contain NMDARs (and no AMPARs) and therefore do not conduct current at their resting state. These synapses are termed "silent synapses" and can incorporate functional AMPARs upon LTP induction by triggering rapid AMPAR trafficking (Isaac et al., 1995; Liao et al., 1995). The fast unsilencing during LTP is supposed to enable the neuronal network to quickly encode new memories in form of new patterns of functional connectivity (Chater \& Goda, 2014).

While LTP enhances synaptic strength, long-term depression (LTD) weakens the synaptic connection between two neurons. Typical LTD, as reported for the hippocampus (Dudek \& Bear, 1992), relies on NMDAR-mediated $\mathrm{Ca}^{2+}$ influx and subsequent activation of high affinity $\mathrm{Ca}^{2+-}$ sensors such as calcineurin (Mulkey \& Malenka, 1992; Mulkey et al., 1994). In turn triggered downstream transduction pathways involve dephosphorylation events on target molecules like the AMPAR subunit GluA1, resulting in AMPAR removal via endocytosis or lateral diffusion and hence in decreased synaptic strength (Lee et al., 1998, 2000; Carroll et al., 1999; Beattie et al., 2000).

Thus, both LTP and LTD are dependent on NMDAR activation and lead to altered number of synaptic AMPARs. The direction of plasticity is thought to be mostly guided by the spatiotemporal characteristics of intracellular $\mathrm{Ca}^{2+}$ increase at the postsynapse, while detailed mechanisms underlying the linkage of NMDARs to signaling cascades related to LTP or LTD remain to be clarified (Chater \& Goda, 2014). 


\subsubsection{Synaptic scaling}

Apart from the so far addressed input-specific "Hebbian" forms of synaptic plasticity (Brown et al., 1990) - LTP and LTD - neuronal networks also execute homeostatic plasticity. This is a form of plasticity referring to cell-autonomous reactions to changes in the overall level of network activity (Maffei \& Fontanini, 2009; Vitureira et al., 2012). While LTP and LTD are limited to the stimulated synapse, homeostatic plasticity affects synaptic AMPAR number across the whole neuronal dendrite, thereby preserving relative strength differences between individual synapses (Turrigiano et al., 1998). This phenomenon is termed "synaptic scaling" as it scales up or down all of a neuron's synaptic inputs.

Homeostatic plasticity was first monitored by miniature EPSCs (mEPSCs, postsynaptic response to a spontaneously released synaptic vesicle) in 1998, when Turrigiano et al. showed that cortical culture neurons adjust their synaptic AMPAR levels in response to external changes in activity. Specifically, blocking of neuronal activity (e.g. via TTX incubation) caused scaling up by AMPAR accumulation at excitatory synapses, whereas the stimulation of neuronal networks (i.e., by blocking of inhibitory neurons via $G_{A B A}$ receptor antagonist bicuculline) evoked scaling down (Turrigiano et al., 1998). Interestingly, other studies revealed that chronic bicuculline-treatment of organotypic slice cultures increases excitatory synaptic transmission (Abegg et al., 2004; Krüger et al., 2013). Thus, the phenomenon of synaptic downscaling is not necessarily common.

\subsection{Role of PSD-95 and PSD-93 in neuronal function and plasticity}

\subsubsection{MAGUKs at the postsynaptic density}

Excitatory glutamatergic synapses contain a prominent electron-dense region at the postsynaptic membrane opposite to the presynaptic terminal - the PSD. The PSD mainly consists of four major classes of components, which are (I.) plasma membrane proteins (e.g. GluRs) and specific cell adhesion molecules such as neuroligins (Kennedy, 1997, 2000), (II.) signaling proteins like CaMKII, SynGAP and the tyrosine kinases Src and Fyn (Kennedy et al., 1983; Ellis et al., 1988; Chen et al., 1998; Kim et al., 1998), (III.) cytoskeletal proteins like actin (Kelly \& Cotman, 1978), and (IV.) scaffolding proteins such as the disc-large (DLG) subfamily of membrane-associated guanylate kinases (MAGUKs) (Gomperts, 1996; Sheng \& Kim, 1996). Therefore, the PSD contains neurotransmitter receptors which are associated with signaling and scaffolding proteins to coordinate downstream signaling pathways in response to synaptic input. 
DLG-MAGUKs play a pivotal role in organizing the PSD, mainly by affecting receptor clustering and function (Sheng \& Kim, 1996; Ziff, 1997), and linking receptor activation to postsynaptic signal transduction machineries (Kennedy, 2000). Each family member possesses five protein-protein interaction domains (Cho et al., 1992). Specifically, three $\mathrm{N}$ terminal PSD-95/discs-large/zona occludens-1 (PDZ) domains are followed by one srchomology-3 (SH3) domain and one guanylate kinase (GK) domain, which lacks enzymatic activity. The four DLG-MAGUK family members are: the postsynaptic density protein 95 (PSD95), PSD-93, synapse-associated protein 97 (SAP97) and SAP102. Apart from their common domain structure, the members feature distinct $\mathrm{N}$-terminal motives which can mediate unique functions (Cho et al., 1992; Schlüter et al., 2006).

The NMDAR subunits GluN2A and GluN2B are shown to directly interact with all DLGMAGUK family members via their intracellular C-terminal domain (Kornau et al., 1995; Lau et al., 1996; Niethammer et al., 1996). In contrast, only SAP97 can directly interact with AMPARs via the GluA1 subunit (Leonard et al., 1998; Cai et al., 2002). PSD-95, PSD-93 and SAP102 are linked to AMPARs via transmembrane AMPAR regulatory proteins (TARPs), such as stargazin in case of PSD-95 (Fukata et al., 2005). DLG-MAGUKs are shown to mediate AMPAR trafficking at different developmental stages and exhibit a striking ability to functionally compensate for each other (Sans et al., 2000; Elias et al., 2006).

\subsubsection{Developmental profiles und functions of DLG-MAGUKs}

In the mammalian brain, DLG-MAGUKs show distinct expression patterns throughout neuronal development. SAP102 is highly expressed already at postnatal day 10 (P10), followed by gradually decreasing levels across development (Sans et al., 2000). This is consistent with the important role of SAP102 in immature synapses, primarily regulating NMDAR function and targeting AMPARs in early development (Elias et al., 2008; Chen et al., 2012; Bonnet et al., 2013). Indeed, knockdown (KD) of SAP102 diminishes AMPAR-mediated transmission specifically at immature synapses (Elias et al., 2006). Furthermore, SAP102 has the ability to upregulate itself and thereby compensate for the loss of synaptic AMPARs caused by deletion of PSD-95 (Bonnet et al., 2013) or of both PSD-95 and PSD-93 (Elias et al., 2006). Accordingly, double knockout (DKO) of PSD-95 and SAP102 is lethal (Cuthbert et al., 2007).

Likewise, germline KO of the paralog SAP97 is lethal, mainly due to disturbed cell-cell contact formation throughout embryogenesis (Woods \& Bryant, 1991; Caruana \& Bernstein, 2001). In wildtype (WT) mice, about $50 \%$ of SAP97 levels are already observed at birth, reaching adult levels around P14 (Wang et al., 2006). During synaptogenesis, SAP97 overexpression in vivo increases AMPAR- as well as NMDAR-mediated currents, while conditional KD or overexpression of SAP97 in mature neurons has no effect on glutamatergic transmission or 
neuronal plasticity (Schnell et al., 2002; Schlüter et al., 2006; Howard et al., 2010). Interestingly, similar to SAP102, the overexpression of SAP97 was reported to compensate for AMPAR trafficking deficits in mature PSD-93/95 DKO neurons (Howard et al., 2010). Nevertheless, the effects of SAP97 overexpression seem inconsistent when considering the enhanced surface AMPAR levels and mEPSC frequency observed in a different study using primary cortical cultures (Rumbaugh et al., 2003).

In contrast to SAP102 and SAP97, PSD-95 and PSD-93 show very low expression levels at neonatal stages with a subsequent steep increase between $\sim$ P10-P35 as reported for hippocampus (Sans et al., 2000). Interestingly, studies on primary visual cortex (V1) showed a sharp increase directly after eye opening and in parallel to silent synapse maturation (D. Favaro et al., in press; Huang et al., 2015a).

Collectively, the different developmental profiles likely suggest distinct functions of DLGMAGUK proteins depending on experience-dependent maturational states and of neuronal networks. Importantly, the expression levels of PSD-95 and PSD-93 indicate specific roles in neuronal network refinement throughout development.

\subsubsection{PSD-95}

PSD-95 exhibits particularly high abundance at the PSD of excitatory synapses and is the most extensively studied DLG-MAGUK family member (Cho et al., 1992; Kistner et al., 1993; Peng et al., 2004). Its predominant palmitoylated a-isoform was shown to regulate synaptic AMPAR number and thereby excitatory synaptic strength (Elias et al., 2006; Schlüter et al., 2006). Specifically, the expression of PSD-95 is positively correlated with AMPAR-mediated EPSCs: While acute KD (Nakagawa et al., 2004; Elias et al., 2006; Schlüter et al., 2006) or genetic deletion of PSD-95 (Béique et al., 2006; Carlisle et al., 2008) diminishes AMPARmediated synaptic currents to $~ 50 \%$, AMPAR-transmission is enhanced two- to threefold upon synaptic PSD-95 overexpression (Schnell et al., 2002; Ehrlich \& Malinow, 2004; Nakagawa et al., 2004; Schlüter et al., 2006; Xu et al., 2008). This increase in AMPAR EPSCs is presumably caused by PSD-95-mediated trafficking of AMPARs to synaptic sites, which possibly results in augmented spine size and suggests a "slot" function of PSD-95 for synaptic AMPARs (Schnell et al., 2002; Ehrlich \& Malinow, 2004). Furthermore, N-terminal di-/ multimerization of PSD-95 was found to be essential for its effect on basal AMPAR function (Xu et al., 2008).

In contrast to the marked influence of PSD-95 on AMPARs, NMDAR-mediated EPSCs are largely unaffected by changes in PSD-95 expression (Migaud et al., 1998; Schnell et al., 2002; Ehrlich \& Malinow, 2004; Nakagawa et al., 2004; Elias et al., 2006; Schlüter et al., 2006). However, PSD-95 is essential for the switch of GluN2B- to GluN2A-containing NMDARs during normal synaptic maturation (Elias et al., 2008). 
Studies on the function of PSD-95 in synaptic plasticity revealed that LTP is enhanced and LTD absent upon PSD-95 KO (Migaud et al., 1998; Béïque et al., 2006; Carlisle et al., 2008). Concurrently, overexpression of PSD-95 occludes LTP and enhances LTD (Béïque \& Andrade, 2003; Stein et al., 2003; Ehrlich \& Malinow, 2004). These results seem surprising considering the slot theory of PSD-95 for synaptic AMPARs, which would have suggested exactly opposite effects of PSD-95 levels on LTP and LTD. To unravel this discrepancy, a study using molecular manipulations of PSD-95 provided evidence that the role of PSD-95 in controlling basal AMPAR strength on one side, and affecting LTD on the other, can be dissociated. Specifically, while PSD-95 serves as a structural scaffold protein for tethering AMPARs at synaptic sites during basal transmission, it operates as a signaling scaffold to connect NMDAR-dependent $\mathrm{Ca}^{2+}$ influx to downstream enzymatic machineries required for LTD generation (Xu et al., 2008). This theory is consistent with the reported direct interaction of PSD-95 PDZ-domains with NMDAR subunits (Kornau et al., 1995; Niethammer et al., 1996), while not influencing NMDAR-mediated EPSCs.

Comparable functions of PSD-95 are indicated for synaptic scaling. Indeed, it has been shown that PSD-95 levels do not directly determine quantal amplitude, but rather serve as a key synaptic organizer to mediate accumulation or reduction of synaptic AMPARs during homeostatic regulation of postsynaptic strength (Sun \& Turrigiano, 2011).

\subsubsection{PSD-93}

In contrast to PSD-95, much less evidence is available on the synaptic function of PSD-93. Interestingly, conflicting results on PSD-93 function were gained in different brain regions and, to some extent, different developmental states:

In the cerebellum, PSD-93 appears to have no effect on structure and function of Purkinje neurons (McGee et al., 2001; Liaw et al., 2008), whereas studies on forebrain cortex and spinal cord revealed impaired NMDAR-mediated EPSCs due to NMDAR surface reduction upon PSD-93 KO (Tao et al., 2003; Liaw et al., 2008). Consistent throughout these studies is unaltered AMPAR-mediated synaptic transmission. In the visual cortex, NMDAR-mediated EPSCs are unaffected upon PSD-93 KO during critical period, whereas AMPAR/NMDAR ratio is increased (critical period will be examined in detail in section 1.5.6) (D. Favaro et al., in press).

On the contrary, acute $\mathrm{KD}$ and overexpression experiments in hippocampal cultures suggest that PSD-93 has PSD-95-like functions. According to Elias et al. (2006), RNA interference (RNAi)-mediated KD of PSD-93 in organotypic slice cultures reduces AMPAR-mediated transmission by $\sim 50 \%$, while overexpression of PSD-93 strongly enhances AMPAR function. In contrast, no influence on NMDAR-mediated EPSCs was observed. Partly opposing results were obtained by genetic KO of PSD-93, which revealed no impairments in basal AMPAR- (as 
well as NMDAR-) mediated transmission in the hippocampus of young mice (Migaud et al., 1998; Elias et al., 2006; Carlisle et al., 2008; Krüger et al., 2013). Notably, Krüger et al. (2013) additionally detected decreased AMPAR/NMDAR ratios in older hippocampal neurons (P6074 mice) and thereby a phenotype similar to KD of PSD-93 in organotypic cultures, which resulted in diminished AMPAR function (Elias et al., 2006).

Collectively, these studies show that function of PSD-93 in basal synaptic transmission seems to be brain region-specific and besides that, PSD-93 may become more relevant to maintain the strength of glutamatergic synapses at later developmental states of hippocampal neurons.

While basal NMDAR-mediated transmission consistently appears unaffected by PSD-93 levels in the hippocampus, PSD-93 deletion elevates the threshold for LTP induction, but has no influence on LTD (Carlisle et al., 2008) - therefore indicating an opposing role as compared to PSD-95 (Migaud et al., 1998; Béïque \& Andrade, 2003).

\subsubsection{Double knockout of PSD-95 and PSD-93}

Mice lacking both PSD-95 and PSD93 display much stronger impairments than single KO mice. Besides smaller body size and low survival rate, DKO mice suffer from hypokinesia and gait abnormalities (Elias et al., 2006). Nevertheless, little is known so far about the underlying molecular mechanisms and how the absence of two dominant DLG-MAGUK proteins influence neuronal function.

Elias et al. (2006) reported that simultaneous KO of PSD-95 and PSD93 results in impaired basal synaptic transmission in the hippocampus by reducing field excitatory postsynaptic potentials (fEPSPs) to $55 \%$ and AMPA/NMDAR ratio to $~ 50 \%$. This observation is similar to the markedly diminished AMPAR EPSCs in PSD-95 KO mice and again highlights the crucial role of PSD-95 in basal synaptic transmission (Béïque et al., 2006; Carlisle et al., 2008). Some more information about synaptic function in DKO mice is provided with respect to silent synapses as addressed in the following paragraphs.

\subsubsection{Silent synapses}

Excitatory glutamatergic synapses typically contain both functional NMDARs and AMPARs at their postsynaptic site. By contrast, nascent or immature excitatory synapses exhibit reliable NMDAR-mediated synaptic transmission, but, either absent or highly labile AMPAR-mediated transmission (Isaac et al., 1995; Liao et al., 1995; Petralia et al., 1999). Therefore, these synapses are functionally dormant - or "silent" - at their resting state, owing to $\mathrm{Mg}^{2+}$-mediated blockage of NMDARs in the absence of sufficient postsynaptic membrane depolarization (Petralia et al., 1999; Kerchner \& Nicoll, 2008). Following NMDAR activation, silent synapses 
can mature (or become unsilenced) by incorporation of functional AMPARs, resulting in LTP of glutamatergic synaptic transmission (Liao et al., 1995; Durand et al., 1996; Isaac \& Crair, 1997; Itami et al., 2003).

While silent synapses abundantly occur during early development and provide important substrates for experience-dependent plasticity, they are not normally enriched in the mature brain (Groc et al., 2006; Kerchner \& Nicoll, 2008). Indeed, the typical window for silent synapse maturation is the critical period, referring to times of enhanced neuronal plasticity, in which experience-dependent cortical network refinement and consolidation of synaptic connections take place (Fagiolini \& Hensch, 2000; Feldman, 2009; Espinosa \& Stryker, 2012). As a result of their highly unstable and plastic nature, silent synapses may not only trigger the formation of new neuronal circuits, but as well the elimination of old neuronal connections due to synaptic pruning (Huang et al., 2015b) - always aiming at optimizing functional connectivity.

Silent synapses can be indirectly detected via AMPAR-mediated mEPSC measurements: While mEPSC amplitude changes are considered to result from changes in AMPAR number or single-channel conductance, mEPSC frequency reflects either changes in presynaptic glutamate release or altered proportion of silent synapses (Béïque \& Andrade, 2003). Additionally, the fraction of silent synapses can be directly determined using the so-called minimal stimulation assay (Liao et al., 1995). In this assay, failure rates of glutamatergic transmission near resting potential (e.g. $-60 \mathrm{mV}$ ) and at depolarized states (e.g. $+40 \mathrm{mV}$ ) are compared. In a neuronal connection with high fraction of silent synapses, the failure rate at $+40 \mathrm{mV}$ will be lower as compared to $-60 \mathrm{mV}$ since both AMPARs and NMDARs are transmitting, including AMPAR-silent synapses.

Interestingly, both PSD-95 and PSD-93 have been linked to silent synapse maturation, but with opposing effects. It is reported that manipulations of PSD-95 levels affect mEPSC frequency, but not the amplitude, indicating selective changes in the number of AMPARpositive synapses, while normal glutamate release is preserved (Béïque \& Andrade, 2003; Stein et al., 2003; Béïque et al., 2006; Carlisle et al., 2008). This phenomenon results from the ability of PSD-95 to trigger silent synapse maturation, presumably by AMPAR recruitment and stabilization, which leads to a higher number of transmitting synapses at resting potential and thus enhanced mEPSC frequency. Accordingly, deletion of PSD-95 causes diminished AMPAR mEPSC frequency and an increased proportion of silent synapses.

Consistently, subsequent studies on diverse brain areas - namely, V1, medial prefrontal cortex (mPFC) and hippocampus - showed that PSD-95 KO mice preserve very high levels of $\sim 50 \%$ silent synapses into adulthood, whereas silent synapse fraction in WT mice is dramatically reduced upon early brain development (Huang et al., 2015; D. Favaro et al., in press). Furthermore, PSD-95-dependent maturation of silent synapses in V1 terminates the critical period for ocular dominance (OD) plasticity (Huang et al., 2015a). OD plasticity is a 
widely used paradigm for experience-dependent cortical plasticity and has a critical period for mice lying between P19 and P32 with peak plasticity at P28, the so-called juvenile period (Hubel \& Wiesel, 1970; Gordon \& Stryker, 1996; Feldman, 2009; Espinosa \& Stryker, 2012). Importantly, PSD-95 KO mice exhibit lifelong juvenile OD plasticity, due to impaired silent synapse maturation (Huang et al., 2015a). Notably, the function of PSD-95 seems to be specific for visual experience-dependent maturation, since silent synapse number already decreased to $\sim 50 \%$ before eye opening, independently of PSD-95.

In contrast to PSD-95, its paralog PSD-93 shows clear inhibiting effects on silent synapse maturation as indicated by increased mEPSC frequency and decreased silent synapse fraction (determined by minimal stimulation assay) in mice lacking PSD-93 (D. Favaro et al., in press). In the absence of PSD-93, silent synapses already mature completely during critical period in V1, reaching zero-levels at P28 (compared to 25\% in WT and 50\% in PSD-95 KO mice). Intriguingly, by loss of PSD-93, the developmental decline of silent synapse fraction is rendered independent of visual experience. Comparable reductions in silent synapse levels are also reported for mPFC and hippocampus, showing $\sim 10 \%$ at $\mathrm{P} 15$ and P20, respectively (D. Favaro et al., in press). In nucleus accumbens, PSD-93 KO mice maintain less than $10 \%$ silent synapses at $\sim$ P28, whereas loss of SAP102 has only limited effects on silent synapse number in this brain area (Shukla et al., 2017).

In mice lacking both paralogs and therefore PSD-95-mediated promoting and PSD-93mediated inhibiting effects on silent synapse maturation, the maturation time course and proportion of silent synapses is similar to WT as reported until late critical period of V1 (P28) (D. Favaro et al., in press). Nevertheless, the maturation is independent of visual experience, indicating a distinct mechanism in the absence of PSD-95 and PSD-93. mEPSC frequency appears normal in DKO mice, but the amplitude is decreased. On one hand, this indicates that DKO mice possess unaltered fraction of AMPAR-transmitting synapses, but on the other hand, reduced AMPAR number at individual excitatory synapses. Interestingly, D. Favaro et al. (in press) also detected reduced mEPSC amplitudes in PSD-95 KO mice in V1. This finding is in contrast to previous studies on mPFC, somatosensory cortex (Béïque \& Andrade, 2003) and hippocampus (Stein et al., 2003; Béïque et al., 2006), all showing unaffected mEPSC amplitudes upon PSD-95 KO. However, increased amplitudes induced by PSD-95 overexpression were reported before in hippocampal cultures (El-Husseini et al., 2000).

Thus, while silent synapses apparently mature in the absence of both PSD-95 and PSD-93, PSD-95 seems to be necessary for normal function of matured excitatory synapses as indicated by normal AMPAR number in mice only lacking PSD-93 (D. Favaro et al., in press). Furthermore, it is shown that both paralogs are necessary for finetuning of visual perception, with either protein promoting visual acuity (D. Favaro et al., in press; Huang et al., 2015a). 
An important question raised by this balancing effect of PSD-95 and PSD-93 on silent synapse maturation is, whether both proteins are actually located at the same synapse. Albeit some studies reported a rather distribution to different synapses in the hippocampus (i.e., just 33\% of co-localization) (Sans et al., 2000) or in hippocampal cultures (Elias et al., 2006), others revealed similar, homogenous distributions and co-localization at single isolated PSDs of rat cortex (DeGiorgis et al., 2006) and mouse V1 (D. Favaro et al., in press).

Taken together, these results show opposing roles of the two MAGUKs PSD-95 and PSD-93 in experience-dependent maturation of silent synapses during critical period. This mechanism is essential for developmental finetuning and consolidation of synaptic connections into final wiring patterns, and therefore for optimized brain function.

\subsection{Link of glutamate receptor complexes to neuronal dysfunction}

\subsubsection{Genetic studies link PSD-95 and PSD-93 to neuro- developmental and neuropsychiatric disorders}

As members of the DLG-MAGUK family possess a multidomain structure, they interact with a variety of membrane and intracellular proteins, thereby forming complexes with GluRs to link channel activity to downstream signal transduction pathways. These complexes typically contain diverse interacting proteins such as SAP90/PSD-95-associated proteins (SAPAPs) (Takeuchi et al., 1997), Shanks (Boeckers et al., 2002; Hayashi-Takagi et al., 2015) and neuroligins (Kennedy, 1997, 2000). Importantly, each of these protein families has been implicated in neuropsychiatric or neurodevelopmental disorders by genetic studies. Specifically, SAPAP3 is associated with obsessive-compulsive disorder (Bienvenu et al., 2009), Shank family proteins with autism spectrum disorder (ASD) (Durand et al., 2007; Berkel et al., 2010) and schizophrenia (Gauthier et al., 2010), and neuroligins with ASD (Jamain et al., 2003). The most prevalent MAGUK, PSD-95, was identified in genetic studies of Williams syndrome, representing a neurodevelopmental disorder occasionally referred to as the opposite to ASD in terms of social behavior (Feyder et al., 2010; Asada \& Itakura, 2012). Furthermore, dysregulated expression of PSD-95, PSD-93 and NMDAR subunits was found in post-mortem brain samples from schizophrenic patients (Toro \& Deakin, 2005; Kristiansen et al., 2006). In fact, the gene encoding PSD-93 (namely dlg2) shows an above-average likelihood for spontaneous somatic mutations, which can cause idiopathic forms of schizophrenia (Kirov et al., 2011; Fromer et al., 2014; Erwin et al., 2016). Consistently, abnormal social behavior and cognitive impairments similar to defects seen in ASD and 
schizophrenia, respectively, have been described in PSD-95 KO and PSD-93 KO mice (Nithianantharajah et al., 2013; Shukla et al., 2017; Winkler et al., 2018). It still needs to be clarified how PSD-95- and PSD-93-associated complexes are involved in disease development and if their influence on silent synapse maturation may play an important role therein.

\subsubsection{Involvement of PSD-95 and PSD-93 in NMDAR-mediated excitotoxicity}

PSD-95 and PSD-93 play central roles in orchestrating synaptic function in excitatory neurons, which primarily depend on the neurotransmitter glutamate. Dysfunctions in glutamatemediated neurotransmission have been implicated in various neuropathologies including epilepsy, ischemic stroke, Huntington's, Parkinson's and Alzheimer's disease, dementia and acute brain injury (Dawson \& Dawson, 1996; Liu et al., 2009b; Gardoni et al., 2010; Parsons \& Raymond, 2014). Glutamate concentrations are strictly controlled in the brain and excessive or persistent release results in overactivation of GluRs, which can cause neuronal damage referred to as excitotoxicity (Dawson et al., 1992; Coyle \& Puttfarcken, 1993). Excitotoxicity is primarily mediated via enhanced activation of GluN2B-containing NMDARs, which provokes intracellular $\mathrm{Ca}^{2+}$ overload and triggers $\mathrm{Ca}^{2+}$-dependent toxic downstream signaling cascades. There are two major pathways proposed to be stimulated upon excessive $\mathrm{Ca}^{2+}$ influx and to mediate neuronal cell damage. On one hand, extrasynaptic NMDARs trigger pro-apoptotic cascades mediated by the $\mathrm{Ca}^{2+}$-dependent protease calpain. On the other hand, NMDARdependent $\mathrm{Ca}^{2+-}$ overload induces nitric oxide (NO) production, which can be highly toxic in non-physiological concentrations.

\subsubsection{Extrasynaptic NMDAR/calpain-mediated excitotoxicity}

NMDARs located at extrasynaptic sites are considered to be specifically vulnerable to the activation of proapoptotic signaling cascades (Hardingham \& Bading, 2010). Typically, the $\mathrm{Ca}^{2+}$-dependent protease calpain is involved, which appears to be exclusively activated by extrasynaptic and not by synaptic NMDARs (Xu et al., 2009). While in immature hippocampal neurons, the fraction of extrasynaptic NMDARs reaches up to $75 \%$ of all NMDARs (Tovar \& Westbrook, 1999), it gradually decreases throughout development, but still represents a substantial population in the adult brain (Petralia et al., 2010). Extrasynaptic NMDARs can be activated by glutamate spillover from adjacent synapses or via ectopic glutamate release from close-by located axons, dendrites or glial processes (Matsui et al., 2005; Petralia, 2012). Additionally, malfunction of glutamate transporters (e.g. defective glutamate transport or reverse operation), increased presynaptic glutamate release probability and enhanced 
number/stability of NMDARs can lead to excessive glutamatergic activity at extrasynaptic sites (Parsons \& Raymond, 2014). There is a multiplicity of $\mathrm{Ca}^{2+}$-triggered proapoptotic signaling cascades which may be activated (for review see Hardingham \& Bading, 2010). The bestknown pathway involves calpain, which gets activated upon intracellular $\mathrm{Ca}^{2+}$ overload and results in activation of p38 mitogen-activated protein kinase (MAPK) and subsequent activation of caspases acting as "death signals" by promoting cell apoptosis (Volbracht et al., 2005).

Besides synaptic NMDARs, also NMDARs located at extrasynaptic sites are reported to associate with DLG-MAGUKs (Allison et al., 1998; Petralia et al., 2010). Hence, PSD-95 and PSD-93 might contribute to the molecular mechanism leading to neuronal damage in prominent neurodevelopmental brain disorders and acute brain injury.

\subsubsection{NMDAR/nNOS-mediated excitotoxicity}

A major component of glutamate excitotoxicity is proposed to be mediated by NO. Excessive intracellular $\mathrm{Ca}^{2+}$, channeled primarily by GluN2B-containing NMDARs, can activate the neuronal NO synthase (nNOS), leading to NO production and derivates with harmful freeradical character. Specifically, nNOS gets activated via $\mathrm{Ca}^{2+} /$ calmodulin and converts Larginine to citrulline and NO (Dawson et al., 1992; Garthwaite \& Boulton, 1995). Under normal physiological conditions, NO has an important role as a second messenger in the CNS including diverse forms of synaptic plasticity (Schuman \& Madison, 1994). However, high concentration of NO can be neurotoxic (Dawson et al., 1992). The critical aspect about NO as a second messenger is, that it does not bind to membrane-associated receptors at discrete loci like conventional neurotransmitters - but is membrane-permeable and can diffuse among neurons to directly act on intracellular constituents. In addition, conventional neurotransmitters are inactivated upon reuptake or enzymatic degradation, whereas NO loses its activity only after chemical reaction with a substrate. Hence, neurons can hardly regulate NO concentration and activity, solely by the control of NO synthesis (Dawson \& Dawson, 1996).

Following NO overproduction, most of the neurotoxic pathways activated are triggered by the highly reactive oxidant peroxynitrite (ONOO-), the reaction product of $\mathrm{NO}$ and a superoxide free radical $\left(\mathrm{O}_{2}-{ }^{-}\right)$, which can also be generated by nNOS (Pou et al., 1992). The activated downstream pathways are diverse and can involve activation of glyceraldehyde-3-phosphate dehydrogenase (GAPDH), poly (ADP-ribose) polymerase (PARP) and nucleases. As a result, severe damage to cellular components, including DNA, proteins and lipids, as well as modulated gene transcription may lead to neuronal apoptosis and/or parthanatos (i.e., PARPdependent cell death) (Dawson \& Dawson, 2018).

Importantly, PSD-95 and - to a lesser extent - PSD-93 have been implicated to tether nNOS to NMDARs and therefore mediate NO synthesis. Specifically, like the MAGUKs, nNOS 
contains a PDZ domain, which can interact with PDZ repeats of PSD-95 or PSD-93. These MAGUKs in turn bind to the cytosolic C-terminus of GluN2 subunits and thereby effectively couple nNOS to NMDAR-mediated $\mathrm{Ca}^{2+}$ influx (Brenman et al., 1996; Stricker et al., 1997; Sattler et al., 1999). The co-expression of PSD-95 with nNOS has been shown in the developing and mature nervous system (Brenman et al., 1996), as well as the formation of a ternary GluN2B-PSD-95-nNOS complex as co-immunoprecipitated from rat forebrain (Christopherson et al., 1999). For PSD-93, co-localization and interaction with both NMDAR subunit and nNOS in the processes of cultured cortical neurons have been reported (Xu et al., 2004).

Glutamate-mediated excitotoxicity via NO synthesis has been especially implicated in ischemia and, more specifically, ischemic stroke. Ischemic stroke refers to severely reduced or completely blocked cerebral blood flow, which results in the shortage of nutrients and oxygen, leading to near-immediate physical and neurological deficits.

The inhibition of nNOS activity by genetic manipulations or pharmacological tools has been shown to prevent NMDAR-mediated excitotoxicity in primary cortical cultures (Dawson et al., 1991; Dawson \& Dawson, 1996) and, furthermore, to reduce infarct volumes and neurological deficits in mice subjected to cerebral ischemia (Huang et al., 1994). Consistent with the presumed involvement of PSD-95 in coupling NMDAR activity to NO toxicity, antisense RNAmediated KD of PSD-95 in cortical cultures attenuates both NO production and excitotoxicity with each of them selectively mediated by NMDARs (Sattler et al., 1999). Furthermore, studies found modified or induced NMDAR-PSD-95-nNOS interactions upon cerebral ischemia in rodent hippocampus (Takagi et al., 2000; Zhou et al., 2010). These observations led to the design and synthesis of interfering peptides and small molecules aimed to inhibit PDZ domainmediated interactions in the ternary NMDAR-PSD-95-nNOS protein complex and thereby uncoupling NMDAR overactivation from toxic NO synthesis (see Figure 1). Notably, the exact molecular mechanisms how and with which targets these compounds interact are controversially discussed and still unclear (Cui et al., 2007; Lai et al., 2014; Bach et al., 2015). Moreover, given the structural similarity of PSD-95 with PSD-93 (Cho et al., 1992), it is likely that these peptides can also disrupt the interaction of PSD-93 with NMDAR or nNOS, thereby questioning PSD-95 as the in vivo target.

However, irrespective of its mechanism of action and targeted protein interaction, the so-called PSD-95 inhibitor Tat-NR2B9c (NA-1) has been shown to provide protection against ischemic stroke in rodents (Aarts et al., 2002; Sun et al., 2008; Jones, 2011) and high-order primates, i.e. macaques, (Cook et al., 2012a, 2012b) by reducing infarct volumes and preserving neurological functions in neuro-behavioral tests. In these studies, stroke was typically induced via middle cerebral artery occlusion (MCAO). NA-1 was also already tested in a phase II clinical trial of ischemic stroke occurring after endovascular aneurysm repair, showing fewer infarcts in inhibitor-treated patients, but no difference in lesion volumes (Hill et al., 2012). 
Besides NA-1, other potential inhibitors were designed, and some (namely ZL006 and Tat- $N$ dimer) as well appear to reduce cerebral ischemic injury in mouse and rat stroke models (Zhou et al., 2010; Bach et al., 2012).

A

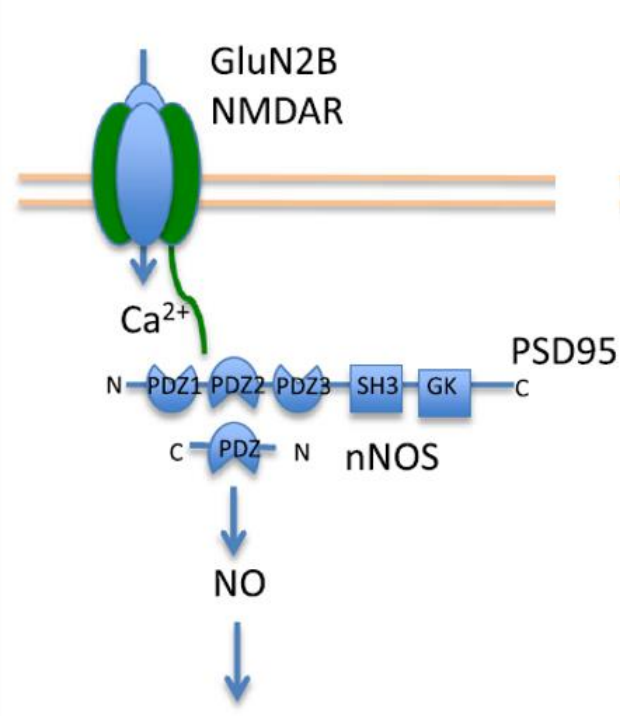

Cell death
B

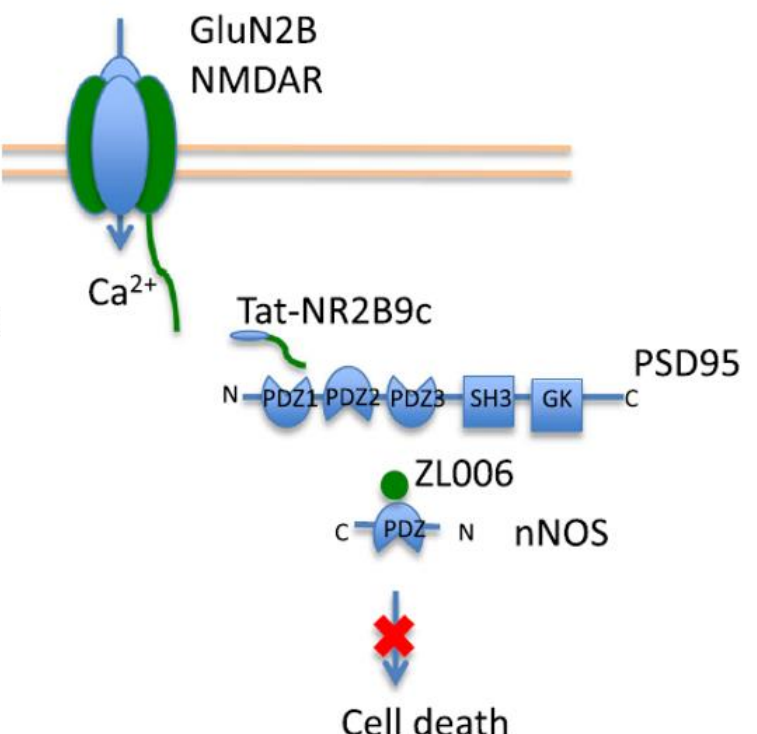

Figure 1: Link between NMDARs and nNOS-mediated neurotoxicity through PSD-95. (A) The scaffolding protein PSD-95 tethers NO-producing nNOS in close proximity to $\mathrm{Ca}^{2+}$ influx channeled by GluN2B-containing NMDARs. Receptor overactivation, e.g., due to excessive glutamate release in cerebral ischemia, leads to $\mathrm{Ca}^{2+}$ overload, massive $\mathrm{NO}$ production and activation of toxic downstream cascades. (B) Interfering peptides including Tat-NR2B9c (also named as NA-1) and small molecules like ZL006 are proposed to perturb PSD-95 interactions with GluN2B and nNOS, respectively. Hence, inefficient signaling reduces NO levels and protects the neuron from cell damage. The exact mechanism how these effector molecules function is still under debate (Cui et al., 2007; Bach et al., 2015). Image adapted from (Wu \& Tymianski, 2018).

In the meantime, some studies focused on a possible role of PSD-93 in mediating NMDAR/nNOS-dependent excitotoxicity. Xu et al. (2004) reported the co-localization and ternary complex formation of PSD-93 with NMDAR and nNOS in cultured cortical neurons. Furthermore, this study showed that PSD-93 deletion prevents NMDAR/nNOS-dependent toxicity induced by platelet-activating factor (PAF) in cortical neurons. PAF is an important proinflammatory cytokine which augments presynaptic glutamate release (Clark et al., 1992) and is considered to be a critical mediator for neuronal injury in the context of ischemia, seizures and brain trauma (Lindsberg et al., 1991; Yue \& Feuerstein, 1994). However, the KO of PSD-93 did not provide neuroprotection in neonatal mice subjected to hypoxia-induced ischemia, but rather enhanced the expression of PSD-95, nNOS and GluN2A/B subunits in the affected hippocampal region (Jiang et al., 2003). In contrast, synaptic GluN2A/B levels were 
reduced in cortical cultures after KD or KO of PSD-93, which additionally decreased $\mathrm{Ca}^{2+}$ influx through NMDARs and, more importantly, attenuated NMDAR-mediated neurotoxicity (Zhang et al., 2010). The indicated protective effect of loss of PSD-93 against NMDAR overactivation is further supported by a mouse MCAO stroke model, which showed reduction in infarct size, neurological deficits and neuronal degeneration when PSD-93 was knocked out (Zhang et al., 2014). This effect was presumed to result from the inhibition of Fyn-mediated GluN2B phosphorylation, which is linked to the neurotoxic cascade following ischemia (Jiang et al., 2008). In this study, the KO of PSD-93 did not increase levels of PSD-95, nNOS and NMDAR subunits after focal cerebral ischemia in cerebral cortex of adult mice (Zhang et al., 2014). Hence, by considering the contradictory effects of PSD-93 disruption in the hippocampus of neonatal mice (Jiang et al., 2003), a conceivable compensatory response of the PSD-95-NMDAR-complex upon PSD-93 KO may depend on brain region, developmental stage and disease model. Deletion of PSD-93 was also shown to inhibit the expression of proinflammatory cytokines in the early stages of ischemia-reperfusion, whereas the mechanism of a possible PSD-93-involved crosstalk between neurons and cytokine-producing glial cells is still unclear (Zhang et al., 2015).

Altogether, these findings suggest important roles for both PSD-95 and PSD-93 in mediating ischemia-induced, NMDAR-dependent neuronal excitotoxicity - possibly through distinct mechanisms and depending on the developmental state. Since some studies are quite controversial and lack mechanistic background, further investigations are needed to specify the role of PSD-95 and PSD-93 in ischemic brain injury and excitotoxicity in general.

\subsection{Spreading depression}

\subsubsection{Characteristics and clinical relevance of spreading depression}

One option to investigate neuronal network susceptibility to metabolic compromise is to induce a transient hypoxic state and characterize the consequences regarding neuronal function and/or damage, e.g., in acute brain slices. Hypoxia represents an important aspect of cerebral ischemia and induces a phenomenon known as "spreading depression" (SD). SD was first described by Leão $(1944,1947)$ as cortical SD of activity due to marked reduction of neuronal activity, spreading out from the addressed area like a wave.

It is first initiated by a profound depolarization of neurons and accompanied by severe reduction of membrane resistance and redistribution of ions across cell membranes, leading to impaired ion homeostasis. Glial cells also depolarize but retain membrane resistance (Somjen, 2001). The severe depolarization leads to massive glutamate release and associated $\mathrm{Ca}^{2+}$ 
overload - a critical excitotoxic state earlier discussed in section 1.6.2. - which shuts down neuronal function and may peak in severe cell damage. During $\mathrm{SD}, \mathrm{K}^{+}$and organic anions, including glutamate, flow out of the neuronal cell and accumulate in the interstitial space (Vyskoeil et al., 1972; Somjen, 2001). Concomitantly, a predominant influx of cations (i.e., $\mathrm{Na}^{+}$ and $\mathrm{Ca}^{2+}$ ) but also $\mathrm{Cl}^{-}$elicit a net gain of solutes, resulting in osmotic cell swelling and reduction of the interstitial space (Somjen \& Aitken, 1984).

Dahlem et al. (2003) described the propagation of SD using a simplified model of a reactiontransport mechanism. According to this model, high concentration of a so-called activator in the extracellular space provokes marked membrane depolarization, resulting in autocatalytic release of the activator - potentially glutamate (Van Harreveld, 1978) or K+ (Grafstein, 1956). Both lead to the efflux of $\mathrm{K}^{+}$ions, which are partially cleared by reuptake, diffusion and redistribution throughout the gap junction-coupled glial network. Hence, high $\mathrm{K}^{+}$concentrations in adjacent cell groups initiate the same excitation cycle and as a result, an excitation wave propagates along the neuronal tissue, followed by sustained depression of activity.

In the brain, SD is usually initiated at a point source and then propagates in gray matter with a velocity of 2-5 mm/min (Marshall, 1959; Lauritzen, 1987). SD waves have been linked to the pathophysiology of ischemic stroke (Hossmann, 1996; Dohmen et al., 2008), where they are thought to account for the severity and extent of neuronal lesions (Nedergaard \& Hansen, 1993; Gido et al., 1994; Hossmann, 1994; Mies, 1997). Typically, adjacent brain tissue not affected by SD spread remains largely healthy (Andrew et al., 1999). Importantly, normoxic SD associated with migraine aura does not cause discernible neuronal damage (Lauritzen, 1994; Bolay et al., 2002). Furthermore, SD is supposed to play a role in concussion, postictal depression (after epileptic seizure), traumatic brain injury and subarachnoid as well as spontaneous/traumatic intracerebral hemorrhage (Strong et al., 2002; Rubaj et al., 2003; Fabricius et al., 2006; Rogawski, 2008; Dreier et al., 2009; Lauritzen et al., 2011).

Interestingly, neuronal insults following hypoxia-induced spreading depression (HSD), e.g. during ischemic stroke, are found to predominantly affect the hippocampal part of the brain (Schmidt-Kastner \& Freund, 1991). Due to its selective vulnerability, the hippocampus serves as a widely-used model structure to study ischemia or SD in general and will also serve as a model in this study. Notably, susceptibility to ischemia differs substantially when comparing discrete subregions of the hippocampus. While CA1 pyramidal neurons suffer from ischemic damage, the adjacent CA3 subfield and dentate gyrus remain mostly unaffected. In addition, interneurons appear quite resistant (Schmidt-Kastner \& Freund, 1991). 


\subsubsection{Spreading depression-associated intrinsic optical signal}

SD can be characterized and identified by electrical as well as optical signals. The marked membrane depolarization of neurons and glial cells is usually accompanied by sudden negative field potential transients of $10-30 \mathrm{mV}$, which represent the electrophysiological hallmark of SD (Aitken et al., 1998; Müller \& Somjen, 2000a). Furthermore, changes in light reflectance of the tissue can be detected and used to map SD spread. As they result from endogenous biological properties, they are termed "intrinsic" optical signals (IOSs).

The characteristic IOS profile was first defined in isolated chick retina by Martins-Ferreira and de Castro (1966). Briefly, a short and weak decrease in tissue reflectance is followed by an abrupt and intense increase, which typically contains two maxima. A large number of studies aimed at identifying the molecular mechanisms which account for the IOS but, so far, they are not completely understood. However, the IOS is supposed to arise mainly from four dominant aspects: (I.) modified light scattering due to volume changes of neurons, glial cells and their corresponding organelles, (II.) changes in blood volume resulting from the lack of energy sources, (III.) activity-related changes in the oxygenation of hemoglobin and (IV.) modified absorption or fluorescence of intrinsic chromophores including cytochromes and cytochrome c-oxidases in mitochondria (Chance et al., 1962; Jöbsis et al., 1977; Mayevsky \& Chance, 1982; Andrew et al., 1999; Kawauchi et al., 2008). Cell swelling and shrinkage appear more pronounced in neuronal dendrites and glial processes than in somatic regions and are thought to trigger neuronal damage following excitotoxic and traumatic brain injury (Somjen, 2001; Rungta et al., 2015).

\subsection{The hippocampal formation}

The hippocampus is probably the most studied region in the brain due to its well-structured and defined neuronal composition. Together with the adjacent amygdala, it forms the central axis of the limbic system. The hippocampus is crucial for the formation and long-term storage of certain types of memories including episodic and spatial memory (Squire, 1992; Neves et al., 2008). Due to its interaction with emotion-related brain regions, the hippocampus is furthermore implicated in emotional behavior (Sahay \& Hen, 2008). A prominent feature of hippocampal synapses is activity-dependent synaptic plasticity. Therefore, and owing to its well-defined anatomical structure, it serves as a major experimental model for the study of diverse neuronal functions using electrophysiological recordings.

The hippocampal formation comprises four cortical regions, namely the dentate gyrus, the hippocampus proper - consisting of Cornu Ammonis subfields 3, 2 and 1 (CA3, CA2 and CA1, respectively), the subiculum and the entorhinal cortex, with the latter subdivided into medial and lateral parts (Figure 2) (Amaral \& Witter, 1989). The cross-section of the hippocampus 
has a defined laminal organization and depth-layers, in which pyramidal cells are arranged. Neuronal connections typically follow this laminar structure, are unidirectional and form closed loops originating mainly in the adjacent entorhinal cortex. The so-called "excitatory tri-synaptic pathway" describes information flow from the entorhinal cortex via the dentate gyrus to hippocampal subfields CA3 to CA1 (Figure 2, Neves et al., 2008). The major neuronal input arrives via the perforant pathway from axons mainly arising in layer II and III (and to a lesser extent in IV and V) of the entorhinal cortex, which projects to granule cells of the dentate gyrus, and pyramidal cells of CA3, CA1 and the subiculum. The axons of dentate gyrus granule cells (called mossy fibers) connect to CA3 pyramidal cells, which in turn project to the CA1 region through Schaffer collaterals. These inputs either arise from CA3 of the same hippocampus (ipsilateral; associational fibers) or the hippocampus of the other hemisphere (contralateral; commissural fibers). Finally, CA1 neurons send out their axons, which pass the subiculum and project onto deeper layers of the entorhinal cortex, representing the principal hippocampal output structure (Lavenex \& Amaral, 2000; Neves et al., 2008).

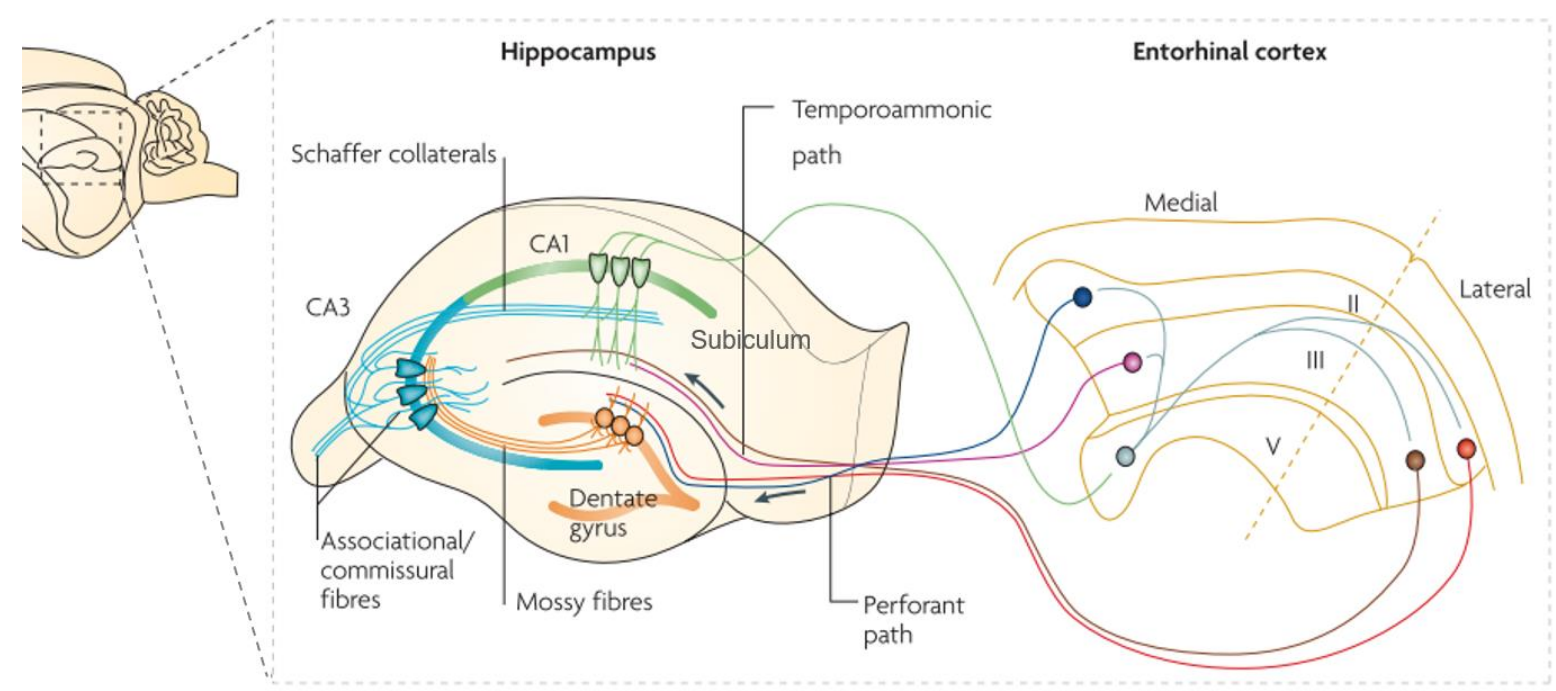

Figure 2: The neuronal circuitry in the rodent hippocampus. The excitatory tri-synaptic loop includes three major pathways: (I.) The perforant path connects the entorhinal cortex to all fields of the hippocampus proper, including granule cells of the dentate gyrus and pyramidal cells of CA3, CA1 and the subiculum, (II.) the mossy fiber pathway conveys information from the dentate gyrus to CA3 neurons, and (III.), CA3 pyramidal cells project to CA1 pyramidal cells through Schaffer collaterals. CA1 neurons can as well receive synaptic input from the contralateral CA3 region via the associational/commissural pathway. Distal apical dendrites of CA1 are connected with neurons from Layer III of the entorhinal cortex via the temporoammonic pathway. The hippocampus also comprises a diversity of inhibitory neurons, which are not shown in the figure. CA, Cornu Ammonis. Image adapted from (Neves et al., 2008). 


\subsection{Scope of the thesis}

DLG-MAGUKs, especially PSD-95 and PSD-93, are critical players in the core organization of the PSD and exhibit important roles in orchestrating synaptic function at developing and mature excitatory synapses. While PSD-95 is well-known to regulate the number of AMPA receptors at the postsynapse and therefore directly affect synaptic strength, changes in PSD95 expression have no influence on NMDAR-mediated transmission (Migaud et al., 1998; Schnell et al., 2002; Elias et al., 2006; Schlüter et al., 2006). Nevertheless, PSD-95 is capable of interacting with NMDAR subunits (Kornau et al., 1995). This direct interaction between receptor and the - so far commonly known as - "structuring" protein has been linked to NMDAR-dependent synaptic plasticity, thereby assigning an additional role to PSD-95, which exceeds its role as a structuring molecule (Migaud et al., 1998; Béïque \& Andrade, 2003; Stein et al., 2003). Specifically, PSD-95 is assumed to serve as a mediator between NMDAR activation and downstream signal transduction pathways required for the induction of synaptic plasticity (Xu et al., 2008).

On the contrary, studies on PSD-93 function in basal synaptic transmission are controversial, presumably because the role of PSD-93 depends on brain region and developmental state (Elias et al., 2006; Carlisle et al., 2008; Krüger et al., 2013). Like PSD-95, PSD-93 can interact with NMDARs, but does not regulate basal NMDAR-mediated transmission in the hippocampus. However, studies indicate contrary roles of PSD-93 in synaptic plasticity as compared to PSD-95 (Migaud et al., 1998; Béïque \& Andrade, 2003), while the molecular mechanisms for either similar, or opposing, functions of the two paralogs still need to be clarified.

\subsubsection{Can the absence of PSD-95 and/or PSD-93 provide protection against hypoxic insults?}

The interaction of PSD-95 and PSD-93 with NMDARs has been implicated in neuronal excitotoxicity - a state, which is linked to diverse neuropathologies including one of the leading causes of long-term disability and death worldwide: ischemic stroke (Lai et al., 2014). Interestingly, while the functions of the two paralogs appear to differ with respect to synaptic transmission and plasticity, both PSD-95 and PSD-93 have been suggested to provide neuronal protection in the context of excitotoxicity (Huang et al., 1994; Sattler et al., 1999; Zhang et al., 2010, 2014). Nevertheless, some studies obtained conflicting results, especially for the role of the less studied paralog PSD-93 (Jiang et al., 2003). Despite the lack of studies on PSD-95 KO mice, showing a protective effect against excitotoxicity due to germ-line KO, PSD-95 has already served as a target of newly designed drugs for stroke prevention (Cook et al., 2012a; Hill et al., 2012). Specifically, interfering molecules have been synthesized to 
prevent the complex formation of PSD-95 with NMDAR and an excitotoxicity-mediator, nNOS. However, whether and, if so, how these peptides actually target the ternary complex is still unclear and controversially discussed (Cui et al., 2007; Bach et al., 2015).

Therefore, the main goal of this study is to decipher a potential role of PSD-95 and PSD-93 in mediating toxic pathways in neuronal networks undergoing metabolic compromise. Specifically, I used transgenic adult mice, in which either one paralog (PSD-95 or PSD-93) or both paralogs were knocked out. This genetic approach enables investigation on the individual protein function, but also provides a state in which both proteins are absent, because MAGUKs are partly able to functionally compensate for each other. In order to investigate whether the absence of PSD-93 and/or PSD-95 can be protective against stroke-like conditions, I used a model of transient hypoxia (but with normoglycemia), containing intact neuronal networks, but excluding system factors such as cerebral vascularization. Due to the reported selective vulnerability of the hippocampus to ischemia and hypoxia-induced spreading depression (HSD), I prepared acute hippocampal brain slices of WT and KO mice. To address possible genotype-related effects on initiation and propagation of HSD, I used a combination of extracellular field potential recording and intrinsic optical imaging. The ability to visualize HSD in the whole brain slice enabled spatial and temporal mapping of HSD wave propagation. By concurrently recording of evoked synaptic responses, I could monitor synaptic function during and after the hypoxic state, providing evidence for the occurrence and extent of hypoxia-induced neuronal damage. Furthermore, I examined basal synaptic transmission and short-term plasticity, in order to gain deeper insight in PSD-95 and PSD-93 function in mature neuronal networks.

\subsubsection{How does the synaptic composition change upon KO of PSD-95 and/or PSD-93?}

We have previously shown that PSD-95 and PSD-93 have opposite roles in the experiencedependent maturation of AMPAR-silent synapses during early development, with PSD-95 KO mice maintaining high proportions of silent synapses into adulthood (D. Favaro et al., in press; Huang et al., 2015a). Higher silent synapse number mimics an immature synaptic state and has been shown to preserve certain plasticity capabilities (Huang et al., 2015a). As stroke susceptibility is known to be highly influenced by aging, I included the aspect of altered number of immature synapses following MAGUK KO into my study.

Due to the fact that the exact molecular composition of silent synapses, especially the occurrence and/or localization of non-transmitting AMPARs, is still elusive, I aimed to dissect the synaptic composition of excitatory neurons in MAGUK KO mice. This time, I used cortical brain slices, since the cortex provides more organic material and was shown to be similarly 
affected by MAGUK KO with respect to silent synapse levels compared to hippocampus. A combination of a biochemical protein crosslinking approach, together with immunofluorescence imaging served as tools to address this question. 


\section{MATERIALS AND METHODS}

\subsection{Materials}

The chemicals and reagents used in the present study are listed in Table 1, the devices in Table 2 and software information is provided in Table 3.

Table 1: List of chemicals and reagents.

\begin{tabular}{ll}
\hline Chemical / reagent & Supplier \\
\hline 3-(N-morpholino)propanesulfonic acid & Sigma-Aldrich, St. Louis, USA \\
(MOPS) & \\
5-Fluoro-2'-deoxyuridine (FUDR) & Sigma-Aldrich, St. Louis, USA \\
Agarose & Invitrogen, Carlsbad, USA \\
AMBA, Rotiphorese Gel 30 & Carl Roth, Karlsruhe, Germany \\
Ammoniumchloride (NH ${ }_{4}$ Cl) & Carl Roth, Karlsruhe, Germany \\
Ammonium peroxodisulfate (APS) & Serva, Heidelberg, Germany \\
Bissulfosuccinimidylsuberate (BS3) & AppliChem, Darmstadt, Germany \\
Bovine serum albumin (BSA, Fraction V, & Serva, Heidelberg, Germany \\
pH 7.0) & \\
Bromphenol blue & Carl Roth, Karlsruhe, Germany \\
Calciumdichloride dihydrate (CaCl 2 -2 H2O) & Carl Roth, Karlsruhe, Germany \\
Choline Chloride & Sigma-Aldrich, St. Louis, USA \\
DABCO & Sigma-Aldrich, St. Louis, USA \\
Deoxycholic acid sodium salt (DEO) & Fluka, Buchs, Switzerland \\
Diethylether & Sigma-Aldrich, St. Louis, USA \\
Dimethylformamide (DMF) & Merck, Kenilworth, USA \\
Dithiothreitol (DTT) & Carl Roth, Karlsruhe, Germany \\
DNase I & Sigma-Aldrich, St. Louis, USA \\
dNTP & Bioline, Luckenwalde, Germany \\
Dulbecco's Modified Eagle Medium/Ham's & Sigma-Aldrich, St. Louis, USA \\
F-12 Nutrient Mixture (DMEM/Ham's F12) & \\
EDTA disodium salt (Na2-EDTA) & Carl Roth, Karlsruhe, Germany \\
Ethidium bromide & Carl Roth, Karlsruhe, Germany \\
Ethylene diamine tetraacetic acid (EDTA) & Carl Roth, Karlsruhe, Germany \\
Ethylene glycol tetraacetic acid (EGTA) & Carl Roth, Karlsruhe, Germany \\
Fetal bovine serum (FBS) & Biowside, USA \\
& \\
\hline
\end{tabular}


Gelatine

GeneRuler 100 bp Plus DNA Ladder

Glucose $\mathrm{D}(+)$

Glucose $D(+)$ monohydrate

GlutaMax (L-Alanyl-L-Glutamine)

Glycerol

Glycine

Goat serum

HEPES

Isoflurane 'Forene'

L-Arginine (L-Arg)

L-Aspartate (L-Asp)

L-Cysteine (L-Cys)

L-Glutamate (L-Glu)

L-Lysine monohydrochloride (L-Lys)

Magnesium dichloride hexahydrate

$\left(\mathrm{MgCl}_{2} \cdot 6 \mathrm{H}_{2} \mathrm{O}\right)$

Magnesium sulfate heptahydrate

$\left(\mathrm{MgSO}_{4} \cdot 7 \mathrm{H}_{2} \mathrm{O}\right)$

Mango Taq DNA Polymerase (5 U/ $\mu \mathrm{l})$

MEM vitamin solution

Methanol

Midori Green

MITO+ serum extender

Modified Minimum Essential Eagle (MEM)

medium (w/o L-glutamine)

MOWIOL

Neurobasal Medium (custom-made DMEM/F12)

Neuronal Supplement NS21

Nonident P40 (NP40)

Papain

Paraformaldehyde (PFA)

Phosphate buffered saline (PBS)

(w/o $\mathrm{Ca}^{2+}$ and $\mathrm{Mg}^{2+}$ )

Poly-D-lysine hydrobromide (PDL)

Ponceau $S$ dye

Potassium chloride (KCl)
Carl Roth, Karlsruhe, Germany

Thermo Fischer Scientific, Waltham, USA

Merck, Kenilworth, USA

Carl Roth, Karlsruhe, Germany

Biochrom, Berlin, Germany

Carl Roth, Karlsruhe, Germany

Carl Roth, Karlsruhe, Germany

Sigma-Aldrich, St. Louis, USA

Carl Roth, Karlsruhe, Germany

AbbVie, North Chicago, USA

Carl Roth, Karlsruhe, Germany

Carl Roth, Karlsruhe, Germany

Carl Roth, Karlsruhe, Germany

Carl Roth, Karlsruhe, Germany

Fluka, Buchs, Switzerland

Carl Roth, Karlsruhe, Germany

Sigma-Aldrich, St. Louis, USA

Bioline, Luckenwalde, Germany

Sigma-Aldrich, St. Louis, USA

Sigma-Aldrich, St. Louis, USA

Nippon Genetics Europe, Düren, Germany

BD Biosciences, Franklin Lakes, USA

Sigma-Aldrich, St. Louis, USA

AppliChem, Darmstadt, Germany

Life Technologies, Carlsbad, USA

Homemade (Chen et al., 2008)

Fluka, Buchs, Switzerland

CellSystems, Troisdorf, Germany

Carl Roth, Karlsruhe, Germany

Sigma-Aldrich, St. Louis, USA

Sigma-Aldrich, St. Louis, USA

Sigma-Aldrich, St. Louis, USA

Carl Roth, Karlsruhe, Germany 
Primers

Protease inhibitor cocktail tablet 'cOmplete' (EDTA free)

Proteinase K $(20 \mathrm{mg} / \mathrm{ml})$

Sodium chloride

Sodium dihydrogen phosphate monohydrate

$\left(\mathrm{NaH}_{2} \mathrm{PO}_{4} \cdot \mathrm{H}_{2} \mathrm{O}\right)$

Sodium dodecyl sulfate (SDS)

Sodium fluoride (NaF)

Sodium hydrogen carbonate $\left(\mathrm{NaHCO}_{3}\right)$

Sodium hydrogen phosphate $\left(\mathrm{Na}_{2} \mathrm{HPO}_{4}\right)$

Sodium hydroxide $(\mathrm{NaOH})$

Sodium L-ascorbate

Sodium orthovanadate $\left(\mathrm{Na}_{3} \mathrm{VO}_{4}\right)$

Sodium phosphate dibasic $\left(\mathrm{NaH}_{2} \mathrm{PO}_{4}\right)$

Sodium pyruvate $\left(\mathrm{C}_{3} \mathrm{H}_{3} \mathrm{NaO}_{3}\right)$

Sodium tetraborate decahydrate

$\left(\mathrm{Na}_{2} \mathrm{~B}_{4} \mathrm{O}_{7} \cdot 10 \mathrm{H}_{2} \mathrm{O}\right)$

Sucrose

Tetramethylethylenediamine (TEMED)

Tetrodotoxin (TTX)

Trichlormethiazide (TCM)

Tris

Tris hydrochloride (Tris-HCl)

Triton X-100

Tween 20

Uridine
MPI-EM, Göttingen, Germany

Roche, Basel, Switzerland

Ambion, Foster City, USA

Carl Roth, Karlsruhe, Germany

Sigma-Aldrich, St. Louis, USA

Carl Roth, Karlsruhe, Germany

Sigma-Aldrich, St. Louis, USA

Sigma-Aldrich, St. Louis, USA

Sigma-Aldrich, St. Louis, USA

Carl Roth, Karlsruhe, Germany

Sigma-Aldrich, St. Louis, USA

Sigma-Aldrich, St. Louis, USA

Sigma-Aldrich, St. Louis, USA

Carl Roth, Karlsruhe, Germany

Sigma-Aldrich, St. Louis, USA

Carl Roth, Karlsruhe, Germany

Carl Roth, Karlsruhe, Germany

Abcam, Cambridge, UK

Sigma-Aldrich, St. Louis, USA

Carl Roth, Karlsruhe, Germany

Carl Roth, Karlsruhe, Germany

Carl Roth, Karlsruhe, Germany

Carl Roth, Karlsruhe, Germany

Sigma-Aldrich, St. Louis, USA

Table 2: $\quad$ List of devices.

\begin{tabular}{ll}
\hline Device & Supplier \\
\hline Biofuge stratos, Heraeus & ThermoFischer Scientific, Waltham, USA \\
Bioruptor sonication device & Diagenode, Denville, USA \\
Biosafety cabinet Herasafe & ThermoFischer Scientific, Waltham, USA \\
BlueCube 300 (gel documentation system) & Serva Electrophoresis, Heidelberg, Germany \\
Centrifuges 5417R, 5424, 5804R, 5810R & Eppendorf, Hamburg, Germany \\
Electrophoresis power supply EV2310 & Consort Medical, Hemel Hempstead, UK \\
Electroporation System ECM 399 & Harvard Apparatus, Cambridge, UK
\end{tabular}


FastGene Ultra Cycler Gradient (FG-TC01, Nippon Genetics Europe, Düren, Germany PCR cycler)

Incubator

Panasonic Corporation, Osaka, Japan

Incubator C200

Labotect Labortechnik, Rosdorf, Germany

Mastercycler proS, vapo protect (PCR cycler)

Odyssey Infrared Imager (WB scanner)

Eppendorf, Hamburg, Germany

Osmometer Vapor Pressure 5520

LI-COR Biosciences, Lincoln, USA

Wescor, Logan, USA

$\mathrm{pH}$ professional PP-15 (pH meter)

Sartorius, Göttingen, Germany

PowerPac 300 (Power supply SDS-PAGE)

Bio-Rad Laboratories, Hercules, USA

Rotator SB3, Stuart (rotation wheel)

Scanvac Coolsafe (freeze dryer)

Shaking Incubator GFL 3032

ThermoFischer Scientific, Waltham, USA

Labogene, Lynge, Denmark

GFL (Gesellschaft für Labortechnik),

Burgwedel, Germany

Thermomixer comfort

Eppendorf, Hamburg, Germany

Thermoshaker TS1

UV-illuminator (gel documentation)

Biometra, Jena, Germany

INTAS Science Imaging Instruments,

Göttingen, Germany

Vibratome VT1200 S

Leica Microsystems, Wetzlar, Germany

\section{Microscopes}

Inverted microscope DM IL

Leica Microsystems, Wetzlar, Germany

LSM 710 confocal microscope

Carl Zeiss Microscopy, Jena, Germany

Objective 40x, oil

Stereo microscope SZX7

Carl Zeiss Microscopy, Jena, Germany

Olympus, Tokio, Japan

\section{Electrophysiological Setup}

Charge-coupled device (CCD) camera

SensiCam Imago QE

Amplifier

Digitizer 1322A (Data Aquisition System)

Imaging Control Unit (ICU)

Light source KL 1500 LCD

Micropipette Puller P-97

Microscope Axiotech, epifluorescent

Objective 5x, 0.13NA Epiplan 442920

Oscilloscope TDS, digital storage

Peristaltic pump 205S

TILL Photonics, Gräfelfing, Germany

Electronics workshop, Physiology, UMG

Axon Instruments, Union City, USA

TILL Photonics, Gräfelfing, Germany

Schott, Mainz, Germany

Sutter Instruments, Novato, USA

Carl Zeiss Microscopy, Jena, Germany

Carl Zeiss Microscopy, Jena, Germany

Tektronix, Beaverton, USA

Watson-Marlow Flow Smart/ Bredel,

Cheltenham, UK 
Polychrome V (imaging system)

Stimulator S88 (photoelectric stimulus

isolation unit PSIU 6)

Temperature controller

Vibroslice 752M
TILL Photonics, Gräfelfing, Germany

Technologies, West Warwick, USA

Electronics workshop, Physiology, UMG

Campden Instruments, Loughborough, UK

Table 3: $\quad$ List of software.

\begin{tabular}{ll}
\hline Software & Company \\
\hline Axon Clampfit 9.2 & Molecular Devices LLC, San Jose, USA \\
Axon pCLAMP 9.2 & Molecular Devices LLC, San Jose, USA \\
Image Studio Lite (2014) & LI-COR Biosciences, Lincoln, USA \\
MetaMorph 6.1 & Universal Imaging, New York, USA \\
SPSS Statistics & IBM, Armonk, USA \\
TILL Vision 4.0 & TILL Photonics, Gräfelfing, Germany \\
ZEN black (2012) & Carl Zeiss Microscopy, Jena, Germany
\end{tabular}

Table 4: List of other material.

Material Company

Borosilicate glass capillaries (type GC150TF- Harvard Apparatus, Holliston, USA

10)

Cyanoacrylate glue (type 401)

Loctite, Düsseldorf, Germany

Microscope coverslips $10 \times 10 \mathrm{~mm}$, round,

Menzel/Thermo Fischer Scientific, Waltham, glass

USA

Microscope slides

Labsolute/Th. Geyer, Höxter, Germany

Stimulation electrode (stainless steel

AM-Systems, Carlsborg, USA

microwire, $50 \mu \mathrm{m}$ diameter) 


\subsection{Laboratory Animals}

\subsubsection{Housing conditions}

Animals used in this study were of the species mus musculus and housed in the animal facility of the European Neuroscience Institute in Göttingen, Germany. The mice were kept in groups up to 5 in macrolon type II L cages $(32.5 \times 17.0 \times 14.0 \mathrm{~cm})$ under standard conditions of a 12 hours light/dark cycle with controlled temperature and humidity. Access to food and water was provided ad libitum. Mice were weaned at 3 weeks of age, followed by separate housing of males and females.

\subsubsection{Genetic background}

Transgenic mouse lines with the background of Charles River C57BL/6J mice (The Jackson Laboratory, Bar Harbor, USA) were backcrossed for at least 10 generations. Experiments were performed with PSD-93 knockout (KO) (McGee et al., 2001), PSD-95 KO (Yao et al., 2004; Abbas et al., 2009) and PSD-93/95 double knockout (DKO) mice. PSD-93 KO and PSD$95 \mathrm{KO}$ mice furthermore contained up to two floxed SAP97 alleles. Floxed SAP97 mice (Zhou et al., 2008) served as controls and are defined as wildtype (WT) mice in the present study.

\subsubsection{Genotyping of transgenic mice}

Animals were genotyped using a protocol according to Malumbres et al. (1997) before and after the experiment. For this purpose, genomic DNA was isolated either from a small tail biopsy ( 1 to $3 \mathrm{~mm}$ of the tail tip) taken after weaning, or from ear punches. The biopsies were lysed in PBND buffer (10 mM Tris, $50 \mathrm{mM} \mathrm{KCl,} 2.5 \mathrm{mM} \mathrm{MgCl} 2 \cdot 6 \mathrm{H}_{2} \mathrm{O}, 0.1 \mathrm{mg} / \mathrm{ml}$ gelatine, $0.45 \%(\mathrm{v} / \mathrm{v})$ Nonident P40 (NP40) and 0.45\% (v/v) Tween 20; $\mathrm{pH}$ 8.3) supplemented with $1: 100$ Proteinase $\mathrm{K}$ solution $\left(20 \mathrm{mg} / \mathrm{ml}\right.$, Ambion) at $55^{\circ} \mathrm{C}$ for at least 3 hours with constant shaking. The enzyme was inactivated by incubation at $99^{\circ} \mathrm{C}$ for $10 \mathrm{~min}$ and the supernatant collected and stored at $4^{\circ} \mathrm{C}$ after centrifugation (14680 rpm for 2 minutes).

Genotypes were determined by polymerase chain reaction (PCR) (Mullis et al., 1986; Saiki et al., 1988) and subsequent agarose gel electrophoresis. The gene specific PCR conditions and primers are listed in Table 5 to Table 8. 10x TNK (Tris (T), ammonium (N), potassium (K)) buffer contained $100 \mathrm{mM}$ Tris, $15 \mathrm{mM} \mathrm{MgCl} 2 \cdot 6 \mathrm{H} 2 \mathrm{O}, 500 \mathrm{mM} \mathrm{KCl}$ and $50 \mathrm{mM} \mathrm{NH} 4 \mathrm{Cl}(\mathrm{pH}$ 8.5). 
Table 5: PCR conditions for SAP97 genotyping.

\begin{tabular}{|c|c|c|c|c|c|}
\hline PCR mixture & & PCR programme & & & \\
\hline & & Cycle step & Temp. & Time & Cycles \\
\hline 10x TNK buffer & $2.2 \mu \mathrm{l}$ & & & & \\
\hline $10 \mathrm{mM}$ dNTP & $2 \mu l$ & & & & \\
\hline (Bioline) & & Initial denaturation & $94^{\circ} \mathrm{C}$ & $2 \min$ & \\
\hline $50 \mu \mathrm{M}$ primer 1 & $0.2 \mu \mathrm{l}$ & Denaturation & $94^{\circ} \mathrm{C}$ & $30 \mathrm{~s}$ & \\
\hline $50 \mu \mathrm{M}$ primer 2 & $0.2 \mu \mathrm{l}$ & Annealing & $60^{\circ} \mathrm{C}$ & $45 \mathrm{~s}$ & $35 x$ \\
\hline $\mathrm{ddH}_{2} \mathrm{O}$ & ad $19.8 \mu \mathrm{l}$ & Extension & $72^{\circ} \mathrm{C}$ & $1 \mathrm{~min}$ & \\
\hline Mango Taq DNA & $0.2 \mu \mathrm{l}$ & Final extension & $72^{\circ} \mathrm{C}$ & $10 \min$ & \\
\hline Pol (5 U/ul, Bioline) & & & & & \\
\hline Lysed biopsy & $2 \mu l$ & & & & \\
\hline
\end{tabular}

Table 6: PCR conditions for PSD-95 genotyping.

\begin{tabular}{|c|c|c|c|c|c|}
\hline PCR mixture & & PCR programme & & & \\
\hline & & Cycle step & Temp. & Time & Cycles \\
\hline 10x TNK buffer & $2.2 \mu l$ & & & & \\
\hline $10 \mathrm{mM}$ dNTP & $2 \mu l$ & & & & \\
\hline (Bioline) & & Initial denaturation & $94^{\circ} \mathrm{C}$ & $5 \min$ & \\
\hline $50 \mu \mathrm{M}$ primer 1 & $0.2 \mu \mathrm{l}$ & Denaturation & $94^{\circ} \mathrm{C}$ & $45 \mathrm{~s}$ & \\
\hline $50 \mu \mathrm{M}$ primer 2 & $0.2 \mu \mathrm{l}$ & Annealing & $60^{\circ} \mathrm{C}$ & $45 \mathrm{~s}$ & $35 x$ \\
\hline $\mathrm{dd}_{2} \mathrm{O}$ & ad $19.8 \mu \mathrm{l}$ & Extension & $72^{\circ} \mathrm{C}$ & $1 \mathrm{~min}$ & \\
\hline Mango Taq DNA & $0.2 \mu \mathrm{l}$ & Final extension & $72^{\circ} \mathrm{C}$ & $10 \min$ & \\
\hline Pol (5 U/ $\mu$ l, Bioline) & & & & & \\
\hline Lysed biopsy & $2 \mu l$ & & & & \\
\hline
\end{tabular}


Table 7: PCR conditions for PSD-93 genotyping.

\begin{tabular}{|c|c|c|c|c|c|}
\hline PCR mixture & & PCR programme & & & \\
\hline & & Cycle step & Temp. & Time & Cycles \\
\hline 10x TNK buffer & $2.2 \mu l$ & & & & \\
\hline $10 \mathrm{mM}$ dNTP & $2 \mu l$ & & & & \\
\hline (Bioline) & & Initial denaturation & $94^{\circ} \mathrm{C}$ & $5 \mathrm{~min}$ & \\
\hline $50 \mu \mathrm{M}$ primer 1 & $0.2 \mu \mathrm{l}$ & Denaturation & $94^{\circ} \mathrm{C}$ & $45 \mathrm{~s}$ & \\
\hline $50 \mu \mathrm{M}$ primer 2 & $0.2 \mu \mathrm{l}$ & Annealing & $61^{\circ} \mathrm{C}$ & $1 \mathrm{~min}$ & $35 x$ \\
\hline $50 \mu \mathrm{M}$ primer 3 & $0.2 \mu \mathrm{l}$ & Extension & $72^{\circ} \mathrm{C}$ & $2 \min$ & \\
\hline $\mathrm{dd} \mathrm{H}_{2} \mathrm{O}$ & ad $19.8 \mu \mathrm{l}$ & Final extension & $72^{\circ} \mathrm{C}$ & $10 \min$ & \\
\hline Mango Taq DNA & $0.2 \mu \mathrm{l}$ & & & & \\
\hline Pol (5 U/ $\mu$ l, Bioline) & & & & & \\
\hline Lysed biopsy & $2 \mu \mathrm{l}$ & & & & \\
\hline
\end{tabular}

Table 8: List of genotyping primers.

\begin{tabular}{ll}
\hline Gene & Primer sequence (5'-3') \\
\hline SAP97 & CCT CTA CAG AAT CTG AGT TGG CTC \\
& TAA GAA GAA TCA ACT GGC AAA GGT G \\
\hline PSD-95 & CAG GTG CTG CTG GAA GAA GG \\
& CTA CCC TGT GAT CCA GAG CTG \\
\hline PSD-93 & GTG CGG AAT GTT GTT GTG CAG TG \\
& CAC AAC AGT CTC CAA TAT GGG TCG C \\
& GCC TTC TAT CGA CTT CTT GAC GAG
\end{tabular}

The PCR product with a total volume of $22 \mu \mathrm{l}$ was analyzed by agarose gel electrophoresis after the addition of $10 \mu \mathrm{l}$ loading dye containing $5 \mathrm{mM}$ sodium tetraborate, $60 \%$ glycerol and bromophenol blue. The gel was prepared with $1 \%$ agarose in sodium tetraborate buffer $(1.9 \mathrm{~g} / \mathrm{l}$ $\mathrm{Na}_{2} \mathrm{~B}_{4} \mathrm{O}_{7}$ ) and electrophoresed at constant voltage of $140 \mathrm{mV}$ for approximately 35 minutes. To visualize the DNA, ethidium bromide (Carl Roth) or Midori Green (Nippon Genetics) was added to the gel solution. Exposure under UV light with either the UV-illuminator by INTAS imaging system or the BlueCube 300 (Serva) visualized DNA bands. The respective lengths were determined according to a DNA ladder (GeneRuler 100 bp Plus DNA Ladder, Thermo Scientific). Table 9 shows the resulting band sizes for each genotype. 
Table 9: Genotype specific lengths of DNA bands.

\begin{tabular}{lll}
\hline Gene & WT band [bp] & $\begin{array}{l}\text { Floxed band (SAP97)/ } \\
\text { KO band (PSD-95, PSD-93) [bp] }\end{array}$ \\
\hline SAP97 & 600 & 900 \\
PSD-95 & 255 & 355 \\
PSD-93 & 330 & 750
\end{tabular}

\subsection{Cell culture}

\subsubsection{Dissociated primary neuronal culture}

Solutions for dissociated neuronal culture:

\section{Feeding medium}

Neurobasal medium supplemented with $100 \mathrm{mM}$ L-Arg/L-Lys, $2 \mathrm{mM}$ GlutaMax and Neuronal Supplement NS21 (Chen et al., 2008)

mGBSS solution

$130 \mathrm{mM} \mathrm{NaCl}, 22 \mathrm{mM}$ HEPES, $11 \mathrm{mM} \mathrm{MgCl} 2 \cdot 6 \mathrm{H}_{2} \mathrm{O}, 5 \mathrm{mM}$ glucose monohydrate, $4.9 \mathrm{mM}$ $\mathrm{KCl}, 2.7 \mathrm{mM} \mathrm{NaHCO}_{3}, 1.5 \mathrm{mM} \mathrm{CaCl}_{2} \cdot 2 \mathrm{H}_{2} \mathrm{O}, 0.8 \mathrm{mM} \mathrm{Na}_{2} \mathrm{HPO}_{4}, 0.3 \mathrm{mM} \mathrm{MgSO}_{4} \cdot 7 \mathrm{H}_{2} \mathrm{O}$ and $0.2 \mathrm{mM} \mathrm{NaH}_{2} \mathrm{PO}_{4} \cdot \mathrm{H}_{2} \mathrm{O} ; \mathrm{pH} 7.32$

\section{Enzymatic solution}

$10 \mathrm{ml}$ mGBSS supplemented with $50 \mathrm{mM}$ EDTA disodium salt ( $\mathrm{Na}_{2}$-EDTA, pH 8.0), $11.39 \mathrm{mM}$ L-cysteine, $10 \mathrm{mM} \mathrm{CaCl} \cdot 2 \mathrm{H}_{2} \mathrm{O}, 30 \mu \mathrm{l} 1 \mathrm{~N} \mathrm{NaOH}, 100 \mu \mathrm{l}$ papain and $10 \mathrm{mg} / \mathrm{ml}$ DNase I

\section{Inactivation solution}

$25 \mathrm{mg}$ bovine serum albumin (BSA) and $10 \mathrm{mg} / \mathrm{ml}$ DNase I in $10 \mathrm{ml} 5 \%$ serum medium I

\section{$5 \%$ Serum medium I}

Neurobasal medium supplemented with $21 \mathrm{mM}$ glucose, $2 \mathrm{mM}$ GlutaMax, MEM vitamin solution, MITO+ serum extender and $5 \%$ fetal bovine serum (FBS)

\section{FUDR solution}

$41 \mathrm{mM}$ 5-Fluoro-2'-deoxyuridine (FUDR) and $20 \mathrm{mM}$ uridine in $\mathrm{dd}_{2} \mathrm{O}$ 
Dissociated primary hippocampal culture was prepared from postnatal day 0 (P0) WT mice according to Huettner \& Baughman (1986) and Schlüter et al. (2006). Therefore, round glass coverslips (10 $10 \mathrm{~mm}$, Menzel/Thermo Fischer Scientific) stored in $96 \%$ ethanol were treated with $1 \mathrm{M} \mathrm{HCl}$ overnight, washed twice with sterile water, again covered in $96 \%$ ethanol and flamed shortly to dry. Subsequently, 24-well plates were equipped with coverslips and coated with Poly-D-lysine hydrobromide (PDL) overnight at $37^{\circ} \mathrm{C}$ and $5 \% \mathrm{CO}_{2}$. At least 2 hours before neuron plating, coverslips were washed in the plate with sterile water, dried at room temperature (RT) for approximately 30 minutes under the biosafety cabinet and covered with $500 \mu \mathrm{l} /$ well plating medium I (100 mM L-Asp/L-Glu in feeding medium). Newborn mice were anesthetized on ice for several minutes and quickly decapitated. Brains were cooled down in ice-cold mGBSS solution and hippocampi collected using the stereo microscope SZX7 (Olympus). During dissection procedure, the hemispheres were separated with a spatula, meninges removed and the hippocampi carefully isolated from cortex and dentate gyrus. After papain and DNase I guided digestion of the hippocampi in enzymatic solution at $37^{\circ} \mathrm{C}$ and mild rotation (10 rpm) for 30 minutes, the solution was replaced with inactivation solution for short incubation. Subsequently, tissue was gently triturated in $5 \%$ serum medium I by pipetting up and down for at least 10 strokes with a fire-polished Pasteur pipette and the cell suspension excluding remaining tissue chunks filled-up with $5 \%$ serum medium I to $10 \mathrm{ml}$. Following centrifugation at $500 \times \mathrm{g}$ for 5 minutes, the cell pellet was resuspended in $5 \%$ serum medium I and neuronal concentration determined via a Neubauer counting chamber. 80000 cells were plated per well in a total volume of $1 \mathrm{ml}$ and kept in the cell culture incubator $\left(37^{\circ} \mathrm{C}\right.$ and $5 \%$ $\mathrm{CO}_{2}$ ) until use at 15 days in vitro (DIV). Addition of FUDR solution at 4 DIV restricted the growth of astrocytes. Neuronal cultures were fed with fresh astrocyte-conditioned medium (section 2.3.2) at 7 DIV and 13 DIV. 


\subsubsection{Preparation of astrocyte-conditioned medium}

Solutions for conditioned medium preparation:

\section{$5 \%$ Serum Medium II}

Modified Minimum Essential Eagle (MEM) medium (w/o L-glutamine) supplemented with $21 \mathrm{mM}$ glucose, $2 \mathrm{mM}$ GlutaMax, MEM vitamin solution, MITO+ serum extender and 5\% FBS

\section{Plating medium II}

Dulbecco's Modified Eagle Medium/Ham's F-12 Nutrient Mixture (DMEM/Ham's F12) supplemented with $2 \mathrm{mM}$ GlutaMax and NS21

Astrocyte culture was prepared from P0 WT mice and brain dissection conducted according to 2.3.1 "Dissociated primary neuronal culture". This time, murine cortices (not hippocampi) were collected, triturated and plated in medium size cell culture flasks with cells from 2 cortices each in $15 \mathrm{ml} 5 \%$ serum medium II. Once per week, the culture was fed with $5 \%$ serum medium II. In order to generate conditioned medium for feeding of dissociated hippocampal cultures, astrocytes were washed with $5 \mathrm{ml}$ plating medium II and cultured in additional $15 \mathrm{ml}$ for 24 hours in the $\mathrm{CO}_{2}$ incubator at $37^{\circ} \mathrm{C}$. After centrifugation at $1500 \times \mathrm{g}$ for 5 minutes, the supernatant (= conditioned medium) was collected and astrocytes fed with fresh $5 \%$ serum medium II. Between several conditioning steps, astrocyte culture was recovered in $5 \%$ serum medium II for at least 48 hours.

\subsection{Protein biochemistry}

\subsubsection{Protein crosslinking assay}

For chemical crosslinking of surface proteins based on Boudreau et al. (2012), acute brain slices were prepared from mice of different age (P13-P32). Subsequently, cortical brain parts were isolated, incubated with the bifunctional crosslinker BS3 (Bis(sulfosuccinimidyl)suberate, Applichem) and further processed via SDS-PAGE and western blotting (section 2.4.2). 


\subsubsection{Acute brain slice preparation}

Solutions for acute brain slice preparation:

\section{Cutting buffer}

$119 \mathrm{mM}$ choline chloride, $30 \mathrm{mM}$ glucose, $26 \mathrm{mM} \mathrm{NaHCO}_{3}, 7 \mathrm{mM} \mathrm{MgSO}{ }_{4} \cdot 7 \mathrm{H}_{2} \mathrm{O}, 3 \mathrm{mM}$ sodium pyruvate, $2.5 \mathrm{mM} \mathrm{KCl}, 1.3 \mathrm{mM}$ sodium L-ascorbate, $1 \mathrm{mM} \mathrm{CaCl} 2 \cdot 2 \mathrm{H}_{2} \mathrm{O}, 1 \mathrm{mM}$ $\mathrm{NaH}_{2} \mathrm{PO}_{4} \cdot \mathrm{H}_{2} \mathrm{O}$ and $1 \mathrm{mM}$ kynurenic acid (Makino et al., 2011)

\section{Artifical Cerebrospinal Fluid (ACSF)}

$119 \mathrm{mM} \mathrm{NaCl}, 26 \mathrm{mM} \mathrm{NaHCO}_{3}, 11 \mathrm{mM}$ glucose, $2.5 \mathrm{mM} \mathrm{KCl}, 2.5 \mathrm{mM} \mathrm{CaCl}_{2} \cdot 2 \mathrm{H}_{2} \mathrm{O}, 1.3 \mathrm{mM}$ $\mathrm{MgSO}_{4} \cdot 7 \mathrm{H}_{2} \mathrm{O}$ and $1 \mathrm{mM} \mathrm{NaH} \mathrm{PO}_{4} \cdot \mathrm{H}_{2} \mathrm{O}$

Mice were anesthetized with isoflurane (Abbvie) and decapitated. Following dissection, the brain was cooled down in ice-cold cutting buffer for about 1 minute. After removal of cerebellum and olfactory lobe, the brain was glued to the cutting platform using cyanoacrylate glue (type 401 , Loctite) and supported by a $2 \%$ agarose block. Coronal slices of $400 \mu \mathrm{m}$ were cut using the vibratome VT1200 S (Leica Microsystems) and collected in the recovery chamber filled with ACSF for 30 minutes incubation at $35^{\circ} \mathrm{C}$ under continuous carbogen supply $\left(95 \% \mathrm{O}_{2}\right.$ and $\left.5 \% \mathrm{CO}_{2}\right)$. Thereby, the $\mathrm{pH}$ value was maintained at 7.4 and proper tissue oxygenation assured.

\subsubsection{BS3 crosslinking}

Solutions for BS3 crosslinking:

\section{HEPES buffer}

$147 \mathrm{mM} \mathrm{NaCl}, 100 \mathrm{mM}$ glucose, $20 \mathrm{mM}$ HEPES and $1.2 \mathrm{mM} \mathrm{CaCl} 2 \cdot 2 \mathrm{H}_{2} \mathrm{O} ; \mathrm{pH} 7.4$

\section{BS3 stock solution}

BS3 was dissolved in dimethylformamide (DMF) for preparation of $50 \mathrm{mM}$ stock solution and vacuum dried using the Scanvac Coolsafe (Labogene) centrifuge. Right before the experiment, BS3 was dissolved freshly in water to $2 \mathrm{mM}$ final concentration. 


\section{Lysis buffer}

$15 \mathrm{mM} \mathrm{NaCl}, 1 \mathrm{mM}$ Tris- $\mathrm{HCl}, 1 \mathrm{mM} \mathrm{Na} 2$-EDTA $-2 \mathrm{H}_{2} \mathrm{O}, 1 \mathrm{mM}$ EGTA, $150 \mathrm{mM} \mathrm{NaF}, 1 \mathrm{mM}$ $\mathrm{Na}_{3} \mathrm{VO}_{4}, 1 \%$ NP-40, 0.5\% DEO, 0.1\% SDS and 1 protease inhibitor tablet; $\mathrm{pH} 7.4$

After recovery, slices were transferred into ice-cold HEPES buffer for cortical sample isolation by preparing similar sizes of crosslinked and control samples. Crosslinking of surface proteins was achieved by incubation with $2 \mathrm{mM} \mathrm{BS} 3$ for 30 minutes at $4^{\circ} \mathrm{C}$ during continuous rotation (4 rpm) of the sample tube. For additional 10 minutes, $100 \mathrm{mM}$ glycine was added to quench the reaction. After removal of the supernatant following centrifugation at $14000 \times \mathrm{g}$ for 2 minutes at $4^{\circ} \mathrm{C}$, lysis buffer was added to homogenize the cortex sample via pipetting up and down. For further analysis, the tissue was processed using the Bioruptor sonication device (Diagenode) with cycles of 30 seconds on/off for 10 minutes at $4^{\circ} \mathrm{C}$ and directly prepared for SDS-PAGE and western blot procedure (section 2.4.2).

\subsubsection{SDS-PAGE and Western blotting}

For protein identification and analysis, samples extracted from acute brain slices were applied on a denaturing SDS-PAGE (Shapiro et al., 1967) after 1:1 addition of 2x SDS sample buffer (Sambrook et al., 1989). 4x SDS sample buffer (400 g/l glycerol, $68.2 \mathrm{~g} / \mathrm{l} \mathrm{Tris,} 40 \mathrm{~g} / \mathrm{l} \mathrm{Tris-HCl}$, $80 \mathrm{~g} / \mathrm{l}$ SDS, $0.6 \mathrm{~g} / \mathrm{l}$ EDTA and $0.75 \mathrm{~g} / \mathrm{l}$ bromphenol blue; $\mathrm{pH} 8.5$ ) was diluted and supplemented with $13.5 \mathrm{~g} / \mathrm{DTT}$ and $2.5 \%$ SDS right before the experiment. Freshly prepared samples were boiled at $99^{\circ} \mathrm{C}$ for 5 minutes, directly loaded on the gel or stored at $-20^{\circ} \mathrm{C}$. Before using, frozen samples were heated up to $60^{\circ} \mathrm{C}$ for another 5 minutes. A volume of $12 / 28 \mu \mathrm{l}$ protein sample was loaded in pockets of a 15-/10-well comb, respectively. Table 10 shows the composition of SDS gels.

Table 10: Gel composition for SDS-PAGE.

\begin{tabular}{lll}
\hline & $\mathbf{1 0} \%$ Separating gel & $\mathbf{3 . 7 5} \%$ Stacking gel \\
\hline AMBA & $6.7 \mathrm{ml}$ & $1.0 \mathrm{ml}$ \\
$3.5 x$ Bis-Tris buffer, pH 6.7 & $6.0 \mathrm{ml}$ & $0.8 \mathrm{ml}$ \\
$10 \%$ SDS & $100 \mu \mathrm{l}$ & - \\
TEMED & $25 \mu \mathrm{l}$ & $20 \mu \mathrm{l}$ \\
$10 \%$ APS & $60 \mu \mathrm{l}$ & $48 \mu \mathrm{l}$ \\
$\mathrm{ddH}_{2} \mathrm{O}$ & $7.3 \mathrm{ml}$ & $6.2 \mathrm{ml}$
\end{tabular}


Electrophoresis was performed in SDS running buffer (0.05 M MOPS, $0.05 \mathrm{M}$ Tris, $1 \mathrm{mM}$ EDTA and $0.1 \%$ SDS) at $60 \mathrm{~V}$ until the samples reached the interface of the two gel components (approx. $20 \mathrm{~min}$ ) and completed at $150 \mathrm{~V}$ for 45 minutes to 1 hour.

The subsequent protein transfer onto nitrocellulose membranes $(0.2 \mu \mathrm{m}$, GE Healthcare) was achieved via wet western blotting (WB) (Towbin et al., 1992) in an upright chamber. After 2 hours of transfer $\left(250 \mathrm{~mA}, 4^{\circ} \mathrm{C}\right)$ in Tris-glycine buffer $(3 \mathrm{~g} / \mathrm{l} \mathrm{Tris,} 14.4 \mathrm{~g} / \mathrm{l}$ glycine, $20 \%$ methanol) the proteins were stained with Ponceau $S$ dye (Sigma Aldrich) to confirm the successful transfer. The dye was washed away with Tris-Buffered Saline-Tween 20 (TBS-T, $20 \mathrm{mM}$ Tris- $\mathrm{HCl}, 140 \mathrm{mM} \mathrm{NaCl}, 0.1 \%$ Tween 20; $\mathrm{pH}$ 7.6) and unspecific binding sites blocked with $5 \%$ BSA in TBS-T for 30 minutes at RT. After overnight incubation at $4{ }^{\circ} \mathrm{C}$ and constant shaking with the primary antibody (diluted in $2.5 \%$ BSA/TBS-T; Table 11), the membrane was washed 2 times for 10 minutes with TBS-T and incubated with the respective secondary antibody diluted in TBS-T for approximately 1 hour at RT under light exclusion.

Table 11: Antibodies used for immunoblotting.

\begin{tabular}{|c|c|c|c|c|}
\hline Antibody & Host & $\begin{array}{l}\text { Approx. MW of } \\
\text { protein [kDa] }\end{array}$ & $\begin{array}{l}\text { Working } \\
\text { dilution }\end{array}$ & Manufacturer \\
\hline \multicolumn{5}{|c|}{ Primary antibody } \\
\hline GABA-A $\alpha 1$ & mouse & 52 & $1: 2000$ & Neuromab \\
\hline GAPDH & mouse & 38 & $1: 2000$ & Merck Millipore \\
\hline GluA1 & rabbit & 110 & $1: 2000$ & Merck Millipore \\
\hline GluA2 & mouse & 100 & $1: 2000$ & Neuromab \\
\hline Mortalin & mouse & 75 & $1: 8000$ & Neuromab \\
\hline \multirow[t]{2}{*}{ Munc13-1 } & rabbit & 200 & $1: 2000$ & Synaptic \\
\hline & & & & Systems \\
\hline GluN2A & rabbit & 180 & $1: 2000$ & Merck Millipore \\
\hline GluN2B & mouse & 166 & $1: 2000$ & Neuromab \\
\hline \multirow[t]{2}{*}{$\beta$-Tubulin } & rabbit & 55 & $1: 1000$ & LI-COR \\
\hline & & & & Biosciences \\
\hline \multicolumn{5}{|c|}{ Secondary antibody } \\
\hline anti-mouse & goat & & $1: 10000$ & LI-COR \\
\hline IRDye 800CW & & & & Biosciences \\
\hline anti-rabbit & goat & & $1: 10000$ & LI-COR \\
\hline IRDye 680CW & & & & Biosciences \\
\hline
\end{tabular}

*Manufacturer information: Merck Millipore (Burlington, USA), Neuromab (Davis, USA), LI-COR

Biosciences (Lincoln, USA) 
Following additional washing steps ( 3 times for 8 minutes with TBS-T and once with TBS), the membrane was scanned with the Odyssey Infrared Imaging System from LI-COR Biosciences. Protein band quantification was conducted by the Image Studio Lite (LI-COR Biosciences) and Image J software using integrated band intensities.

\subsubsection{Antibody uptake assay}

\section{Antibody feeding buffer}

$139 \mathrm{mM} \mathrm{NaCl}, 10 \mathrm{mM}$ HEPES, $10 \mathrm{mM}$ glucose, $2.5 \mathrm{mM} \mathrm{CaCl}_{2} \cdot 2 \mathrm{H}_{2} \mathrm{O}, 2.5 \mathrm{mM} \mathrm{KCl}, 1.3 \mathrm{mM}$ $\mathrm{MgCl}_{2} \cdot 6 \mathrm{H}_{2} \mathrm{O}$ and $1 \mu \mathrm{M}$ tetrodotoxin (TTX); pH 7.3, 290 mOsm (Fredj \& Burrone, 2009)

Synaptotagmin 1 (Syt1) antibody uptake assay was performed in dissociated primary hippocampal culture (2.3.1 "Dissociated primary neuronal culture") at 15 DIV. Coverslips with hippocampal neurons were placed upside-down onto $50 \mu$-drops of reaction solution containing Syt1 antibody (Table 12) in antibody feeding buffer (see above). Depending on different conditions, the reaction solution was supplemented with $0.5 \mathrm{mM}$ Trichlormethiazide (TCM), $20 \mathrm{mM} \mathrm{KCl}$ (total concentration) or $300 \mathrm{mM}$ sucrose. After incubation at $37^{\circ} \mathrm{C}$ and $5 \%$ $\mathrm{CO}_{2}$ for 10 minutes, the neurons were recovered for 1 minute in conditioned medium and applied to immunofluorescence staining described in the following chapter 2.4.4.

\subsubsection{Immunofluorescence staining}

Solutions for immunofluorescence staining:

\section{Goat Serum Dilution Buffer (GSDB)}

$450 \mathrm{mM} \mathrm{NaCl}, 20 \mathrm{mM}$ Phosphate buffer $\left(\mathrm{NaH}_{2} \mathrm{PO}_{4} \cdot \mathrm{H}_{2} \mathrm{O} / \mathrm{Na}_{2} \mathrm{HPO}_{4}\right)$ and $2 \%$ goat serum; $\mathrm{pH} 7.4$

\section{Fixation solution}

$4 \%$ sucrose and $4 \%$ paraformaldehyde (PFA) in phosphate buffered saline (PBS, w/o Ca ${ }^{2+}$ and $\left.\mathrm{Mg}^{2+}\right) ; \mathrm{pH} 6.9-7.2$

Coverslips with primary neuronal culture were carefully transferred onto another 24-well cell culture plate containing $500 \mu \mathrm{l} /$ well fixation solution and incubated at RT for 15 minutes. The fixative was rinsed twice with $500 \mu$ PBS (w/o $\mathrm{Ca}^{2+}$ and $\mathrm{Mg}^{2+}$ ) followed by blocking and permeabilization using $0.3 \%$ Triton X-100 solution in GSDB at RT for 30 minutes. In general, permeabilization of cell membranes allows detection of intracellular antibodies like the Syt1 
antibody, which was taken up by the neurons. Additionally, intracellular antigens were labeled with primary antibodies in the subsequent overnight $\left(4^{\circ} \mathrm{C}\right)$ incubation of coverslips placed upside-down on $50 \mu \mathrm{l}$-drops of antibody solutions in GSDB. For different primary and secondary antibodies see Table 12.

Table 12: Antibodies used for immunofluorescence staining.

\begin{tabular}{llll}
\hline Antibody & Host & $\begin{array}{l}\text { Working } \\
\text { dilution }\end{array}$ & Manufacturer* \\
\hline Primary antibody & mouse & $1: 5$ & Santa Cruz \\
Ankyrin B & chicken & $1: 2000$ & Biosensis \\
MAP2 & guinea pig & $1: 500$ & Synaptic Systems \\
Munc13-1 & rabbit & $1: 200$ & Synaptic Systems \\
Syt1 (luminal domain) & mouse & $1: 300$ & Synaptic Systems \\
VGLUT1 & & &
\end{tabular}

$\begin{array}{llll}\text { Secondary antibody } & & & \\ \text { anti-chicken Alexa Fluor 647 } & \text { goat } & 1: 1000 & \text { Invitrogen } \\ \text { anti-guinea pig Alexa Fluor 647 } & \text { goat } & 1: 1500 & \text { Invitrogen } \\ \text { anti-mouse Alexa Fluor 488 } & \text { goat } & 1: 2000 & \text { Invitrogen } \\ \text { anti-rabbit Alexa Fluor 555 } & \text { goat } & 1: 1500 & \text { Invitrogen }\end{array}$

*Manufacturer information: Santa Cruz (Santa Cruz, USA), Biosensis (Thebarton, Australia), Synaptic Systems (Göttingen, Germany), Invitrogen (Carlsbad, USA)

Coverslips were then washed 3 times in GSDB (w/o goat serum) at RT for 10 minutes each and once with PBS (w/o $\mathrm{Ca}^{2+}$ and $\mathrm{Mg}^{2+}$ ) for 30 minutes. These and all the following washing steps were carried out during gentle shaking on a rocking plate. Primary antibodies were decorated with the respective secondary antibody conjugated to a fluorescent Alexa Fluor dye at RT for 2 hours under light exclusion. Afterwards, the coverslips were washed 3 times with GSDB (w/o goat serum) for 10 minutes each, once in PBS (w/o Ca ${ }^{2+}$ and $\mathrm{Mg}^{2+}$ ) for 30 minutes and once in $5 \mathrm{mM}$ phosphate buffer $(\mathrm{pH} \mathrm{7.4)}$ for 15 minutes. Coverslip mounting was carried out with approximately $6 \mu \mathrm{l}$ MOWIOL-DABCO mounting medium (10\% MOWIOL, $25 \%$ DABCO, 25\% glycerol and 0.1 M Tris) onto microscope slides (Labsolute/Th. Geyer), which were stored after consolidation at $-20^{\circ} \mathrm{C}$ protected from light. 


\subsubsection{Image analysis and quantification}

Image acquisition was performed with the confocal microscope LSM 710 from Carl Zeiss Microscopy, combined with a 40x magnification oil-objective. Throughout the same experiment, images for every condition were obtained using identical acquisition parameters in ZEN black imaging software (Carl Zeiss Microscopy) and the same analysis parameters for exported raw images in Image J. Each experimental condition was represented by at least 5 coverslips from 3 or more independently prepared hippocampal cultures. For each coverslip, 5-10 neurons were analyzed using 3 dendritic regions of interests (ROls) per neuron. To quantify intracellular Syt1 levels, dendritic ROls of similar size were defined manually in Image $\mathrm{J}$ for fluorescence intensity calculation in this area. Intensity was normalized to the average fluorescence of untreated control samples.

\subsection{Electrophysiology}

\subsubsection{Acute brain slice preparation for electrophysiology}

All electrophysiological recordings were performed in collaboration with the Institute of Neuroand Sensory Physiology (University Medical Center Göttingen, Germany). Recordings were performed in acute hippocampal slices prepared from P60 mice. To this end, mice were decapitated under anesthesia with diethylether and the brains rapidly removed from the skull and cooled down in ice-cold ACSF for 1-2 minutes. ACSF contained $130 \mathrm{mM} \mathrm{NaCl}, 24 \mathrm{mM}$ $\mathrm{NaHCO}_{3}, 10 \mathrm{mM}$ glucose, $3.5 \mathrm{mM} \mathrm{KCl}, 1.2 \mathrm{mM} \mathrm{CaCl} \cdot 2 \mathrm{H}_{2} \mathrm{O}, 1.2 \mathrm{mM} \mathrm{MgSO}{ }_{4} \cdot 7 \mathrm{H}_{2} \mathrm{O}, 1.25$ $\mathrm{mM} \mathrm{NaH} \mathrm{PO}_{4} \cdot \mathrm{H}_{2} \mathrm{O}$ and was constantly aerated with carbogen $\left(95 \% \mathrm{O}_{2}\right.$ and $\left.5 \% \mathrm{CO}_{2}\right)$ for $\mathrm{pH}$ adjustment to 7.4. Coronal slices of $400 \mu \mathrm{m}$ were cut using the vibroslice 752M (Campden Instruments), collected in cold ACSF and the hemispheres separated. Shortly thereafter, slices were placed in an Oslo style interface recording chamber and allowed to recover for around 90 minutes. The interface chamber was maintained at $34-35^{\circ} \mathrm{C}$ and perfused with oxygenated and pre-warmed ACSF at a flow rate of $3-4 \mathrm{ml} / \mathrm{min}$ using a peristaltic pump (type 205S, Watson-Marlow). Slices were constantly supplemented with carbogen at a flow rate of 400 $\mathrm{ml} / \mathrm{min}$ to keep cells alive as long as possible (up to 6 hours). A lid covering the recording chamber with a small closable window to place the electrodes prevented the slices from draining. 


\subsubsection{Extracellular field potential recordings}

Acute hippocampal slices of WT, PSD-95 KO, PSD-93 KO and PSD-93/95 DKO mice were tested at 8-9 weeks of age (i.e., P60) with up to 6 slices being used from each animal. Extracellular field potential recordings were performed according to Müller \& Somjen (1998) and Hepp et al. (2005). Electrodes were prepared from thin-walled borosilicate glass capillaries (type GC150TF-10, outer/inner diameter $1.5 / 1.17 \mathrm{~mm}$, wall thickness $0.17 \mathrm{~mm}$; Harvard Apparatus) using a horizontal micropipette puller (type P-97, Sutter Instruments). The recording electrode was filled with ACSF and its tip carefully trimmed to a resistance of 5$10 \mathrm{M} \Omega$. The stimulating electrode consisted of a stainless steel microwire (50 $\mu \mathrm{m}$ in diameter, AM Systems), which was soldered to a golden pin and thereby connected to the stimulator (S88 stimulator with photoelectric stimulus isolation units PSIU6, Grass Technologies). Position of both electrodes was controlled manually using micromanipulators.

Field excitatory postsynaptic potentials (fEPSPs) were evoked by unipolar stimuli of $0.1 \mathrm{~ms}$ via the stimulating electrode placed at hippocampal CA3-CA1 junction to stimulate the Schaffer collateral (SC) pathway. Evoked orthodromic responses were recorded in st. (stratum) radiatum of CA1 with a sampling rate of $20 \mathrm{kHz}$ using an extracellular direct current (DC) potential amplifier (electronics workshop, Physiology, UMG). The amplifier was controlled through the software Axon pCLAMP 9.2 (Molecular Devices LLC) and signals were visualized on the digital oscilloscope TDS 2014 (Tektronix). The Axon Instruments Digitizer (Digidata 1322A) served as an analogue-to-digital converter between the amplifier and computer.

\subsubsection{Input-output curve}

Synaptic strength between SC and CA1 st. radiatum neurons was detected by measuring the input-output (I-O) curve via extracellular field potential recordings. To this end, SC neurons were stimulated with increasing stimulus intensities of $10-150 \mu \mathrm{A}$ in $10 \mu \mathrm{A}$ increments (= total of 15 different stimuli) and the resulting fEPSPs in CA1 st. radiatum recorded. fEPSP slopes were normalized to presynaptic fiber volley (FV) amplitudes as detected at maximal stimulation intensity. For each stimulus intensity, 4 consecutive traces were pooled and averaged. Electrophysiological analysis was performed using the Axon Clampfit 9.2 (Molecular Devices LLC) software. 


\subsubsection{Paired-pulse ratio}

In order to assess presynaptic neurotransmitter release probability at SC-to-CA1 synapses, paired-pulse ratio (PPR) recordings were performed. PPR is a type of short-term plasticity, in which two consecutive synaptic responses are evoked by paired, short-interval stimulation. In such a recording, the amplitude of the second synaptic response depends on the amplitude of the first, with the ratio of the two responses being assumed to depend on the presynaptic release probability $(\mathrm{Pr})$ (Dobrunz \& Stevens, 1997). The weaker the amplitude of the second fEPSP relative to the first, the higher is the presynaptic release probability (and vice versa). Stimulus intensity was set to the half-maximal response obtained during $1-0$ curve measurements. 2 EPSCs were evoked with paired stimuli of 25-200 ms interpulse intervals in $25 \mathrm{~ms}$ increments (= total of 8 different intervals). The PPR is defined as the ratio of the second EPSP amplitude to that of the first response. Paired pulse depression results in negative PPR values, whereas positive PPRs arise from paired pulse facilitation.

\subsubsection{Hypoxia induced spreading depression}

As mentioned above, viability of acute hippocampal slices was preserved by continuous carbogen supply of the interface chamber $\left(95 \% \mathrm{O}_{2}\right.$ and $\left.5 \% \mathrm{CO}_{2}\right)$ at a flow rate of $400 \mathrm{ml} / \mathrm{min}$. For the induction of spreading depression (SD) according to Gerich et al. (2006), gas supply was switched from carbogen to nitrogen $\left(95 \% \mathrm{~N}_{2}\right.$ and $\left.5 \% \mathrm{CO}_{2}\right)$ to create a severe hypoxic environment in the chamber, whereas carbogen aeration of ACSF was continued during hypoxia. This type of SD is called "hypoxia-induced spreading depression" (HSD). Specifically, oxygen withdrawal in the interface chamber induced HSD within 1-2 minutes and the slice was reoxygenated by switching back to carbogen 1 min, 2 min or 4 min after HSD onset. The HSD-accompanying negative deflection of the extracellular direct current (DC) potential was detected and its time to onset $(\Delta \mathrm{t})$, amplitude $(\Delta \mathrm{V})$ as well as duration $\left(\mathrm{t}_{1 / 2}\right)$ calculated using Axon Clampfit 9.2 (Molecular Devices LLC) software. Along this, the amplitude was measured between pre-hypoxia baseline and the maximal DC potential change by considering only rapid shifts of at least $-10 \mathrm{mV}$ amplitude.

To additionally visualize synaptic function throughout HSD induction and subsequent neuronal recovery, fEPSPs were measured upon continuous stimulation of $100 \mu \mathrm{A}$ and $0.05 \mathrm{~Hz}$ (every 20 s) (Hepp \& Müller, 2008). 


\subsubsection{Intrinsic optical signal}

Concomitantly, the intrinsic optical signal (IOS) resulting from changes in tissue light reflectance was recorded in st. radiatum of CA1 hippocampus. The HSD-accompanied IOS provides information about HSD wave characteristics like its propagation velocity and the invaded hippocampal area. To this end, brain slices were illuminated by green light (460$560 \mathrm{~nm}$ ) (Mané \& Müller, 2012) at an angle of $\sim 45^{\circ}$ (light source KL 1500 LCD, Schott) and monitored using a computer-controlled imaging system (Polychrome V, TILL Photonics) in combination with the sensitive charge-coupled device (CCD) SensiCam camera (Imago QE, TILL Photonics). The camera was attached to an upright epifluorescence microscope (Axiotech, Carl Zeiss Microscopy) using a 5x, 0.13NA objective (Epiplan 442920, Carl Zeiss Microscopy) (Gerich et al., 2006). Images were taken every $2 \mathrm{~s}$ with an exposure time of $15 \mathrm{~ms}$ and reflectance changes visualized offline via image subtraction referring to an image taken before oxygen withdrawal (Müller \& Somjen, 1999). Using a 256 gray-scale mode that covers a range of $\pm 20 \%$ brightness variation, the intensity and temporal profile was analyzed within a rectangular $\mathrm{ROI}$ in $\mathrm{CA} 1$ st. radiatum close to the recording electrode. At the maximal increase in light scattering, tissue area invaded by HSD was calculated by referring to the total hippocampal area. The HSD propagation velocity was determined from the progression of the reflectance increase in CA1 st. radiatum parallel to st. pyramidale. Images were processed and analyzed via TILL Vision 4.0 (TILL Photonics) and MetaMorph 6.1 (Universal Imaging) software.

\subsection{Statistical data analysis}

Details of statistical analysis are provided in the respective figure legends and/or result sections and a summary of all statistical analysis in Table 17 (appendix). Data from repeated runs of the same experiment was pooled and statistically analyzed using two-tailed, unpaired Student's t-test. Comparison between genotype (gt) or age (ag) was tested with two-way analysis of variance (ANOVA) followed by least significant difference (LSD) post-hoc tests using IBM SPSS Statistics software. To additionally describe developmental protein profiles for a specific genotype, separate one-way ANOVAs followed by post-hoc LSD multiple comparison tests were performed. All data is given as mean values \pm standard error of the mean (SEM) and significance is indicated as follows: ${ }^{* *} p<0.001 ;{ }^{* *} p<0.01$ and ${ }^{*} p<0.05$. For biochemical western blot analysis, the sample size $(n)$ is shown as $n=$ number of animals tested. For immunofluorescence staining, $\mathrm{n}$ represents the number of coverslips for one specific condition. The number of acute brain slices analyzed electrophysiologically $(n)$, as well as the number of animals $(\mathrm{m})$ in one group is indicated as $\mathrm{n} / \mathrm{m}$. 


\section{RESULTS}

\subsection{Protective effect of PSD-95 KO against hypoxia- induced synaptic dysfunction}

To investigate if the absence of PSD-93 or PSD-95 - as well as the lack of both proteins simultaneously - can be protective for neuronal networks against metabolic compromise, I performed a hypoxia-induced spreading depression (HSD) procedure in acute brain slices of the respective KO mice. Specifically, I recorded extracellular field potentials (fEPSPs) of hippocampal neurons in st. radiatum of the CA1 subfield in response to electrical stimulation of the Schaffer collateral (SC) - CA1 pathway in the hippocampal CA3-CA1 junction (Figure 3).

Using this approach, I examined genotype differences in basal synaptic transmission and short-term plasticity of the hippocampal circuitry. Additionally, I assessed genotype effects on the network response to severe hypoxia using a combination of electrophysiological and optical parameters.

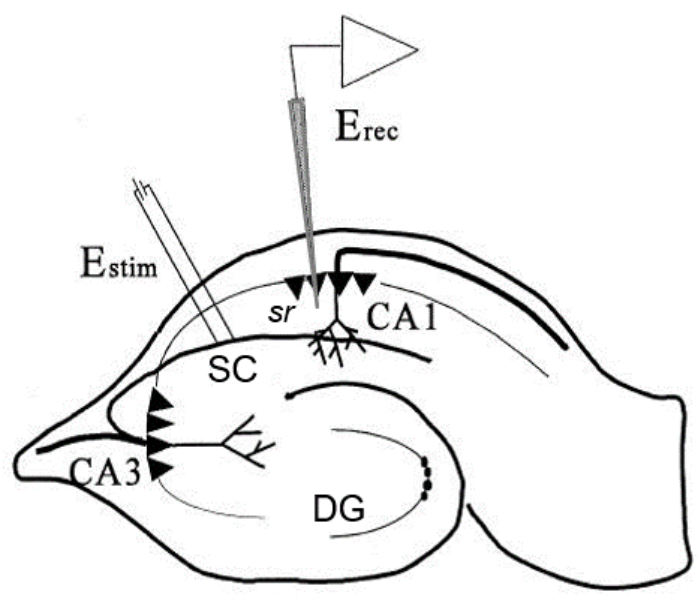

Figure 3: Schematic illustration of fEPSP recordings in the hippocampus. fEPSPs were recorded in CA1 neurons of the st. radium (Erec) and evoked by stimulating the SC pathway ( $\left.\mathrm{E}_{\text {stim }}\right)$. DG, dentate gyrus; sr, stratum radiatum. Image adapted from (Nasir et al., 2010). 


\subsubsection{Altered synaptic transmission and short-term plasticity in MAGUK KO mice}

At first, I tested basal synaptic function of hippocampal neurons in brain slices of WT, PSD-95 KO, PSD-93 KO and PSD-93/95 DKO mice at two months of age (P58-65). fEPSPs were recorded upon single pulse stimulation with gradually increasing current intensities of 10-150 $\mu \mathrm{A}$ using $10 \mu \mathrm{A}$ increments. The resulting input-output (I-O) curves show characteristic progression with increasing fEPSP slopes for higher-intensity stimuli among all genotypes (Figure 4A; values in Table 15 (appendix); stimulus intensity effect: $F_{14,700}=33.078, p<$ 0.001]. However, a genotype effect $\left[F_{3,50}=7.422, p<0.001\right]$ dependent on the stimulus intensity [interaction effect: $F_{42,700}=5.827, p<0.001$ ] was detected. Notably, slices from PSD$95 \mathrm{KO}$ and PSD-93/95 DKO mice displayed lower strength of synaptic transmission compared to control mice as evidenced by fEPSP slope reduction of more than 50\% [PSD-95 KO vs. $W T, p=0.012$; PSD-93/95 DKO vs. WT, $p=0.004$; PSD-95 vs. PSD-93/95 DKO, $p=0.458]$. By contrast, PSD-93 deficient mice were undistinguishable from WT [PSD-93 KO vs. WT, $\mathrm{p}=0.280$ ] and exhibited strongly enhanced responses compared to PSD-95 KO and PSD93/95 DKO mice [PSD-93 KO vs. PSD-95 KO, $p=0.001$; PSD-93 KO vs. PSD-93/95 DKO, $p$ $<0.001]$.

In order to evaluate a form of short-term synaptic plasticity, I measured paired-pulse ratios (PPRs). Specifically, pairs of EPSPs were evoked, from which the PPR was calculated as the amplitude ratio of the second to the first EPSP. The stimulus intensity was adjusted to halfmaximal response, gained from I-O curves and the interpulse interval progressively increased from 25 to $200 \mathrm{~ms}$ in $25 \mathrm{~ms}$ increments (8 steps in total). The resulting PPR is assumed to be inversely correlated with presynaptic release probability $(\mathrm{Pr} r)$ at glutamatergic synapses (Dobrunz \& Stevens, 1997). Upon electrical stimulation, calcium influx into the presynaptic terminal is triggered by the incoming action potential, which promotes vesicle fusion and subsequent neurotransmitter release. In case of low Pr many vesicles are not released and stay docked at the presynaptic membrane after the first action potential occurred. Hence, the residual calcium accumulates with calcium from the second action potential evoked shortly after, leading to elevated vesicle fusion events and a stronger postsynaptic response. This scenario causes increased amplitudes of the second EPSP, rendering synapses with low $\mathrm{Pr}$ more likely to exhibit paired-pulse facilitation (i.e., PPR values greater than one). By contrast, at synapses with high $\operatorname{Pr}$, neuronal depolarization evokes more likely vesicle fusion, so that less vesicles stay docked at the membrane following the first action potential. These synapses typically show smaller amplitudes of the second EPSP and thus exhibit paired-pulse depression, in which the PPR is smaller than one. 
PPR curves of all experimental groups exhibited amplitude ratios above one, thus indicating paired-pulse facilitation (Figure 4B; EPSP2/EPSP1 values are provided in Table 16, appendix). The characteristic decrease in PPR with longer interstimulus intervals $\left[\mathrm{F}_{7,371}=\right.$ 33.046, $p<0.001$ ] can be explained by increasing duration until the second action potential, during which residual calcium can be cleared from the presynaptic terminal, resulting in lower secondary EPSP amplitudes and therefore lower PPRs. Interestingly, PSD-95 KO as well as PSD-93/95 DKO slices exhibited less pronounced facilitation compared to WT and PSD-93 $\mathrm{KO}$ mice $\left[\mathrm{F}_{3,53}=4.789, \mathrm{p}=0.005\right.$; PSD-95 KO vs. WT, $\mathrm{p}=0.015$; PSD-95 KO vs. PSD-93 KO, $p=0.003 ;$ PSD-93/95 DKO vs. WT, $p=0.036$; PSD-93/95 DKO vs. PSD-93 KO, $p=0.009$, all other $p$ values $>0.05]$. The genotype effect was especially strong for short interpulse intervals as indicated by the interaction effect $\left[F_{21,371}=2.107, p=0.003\right]$.

This result implies higher $\mathrm{Pr}$ in mice lacking PSD-95 or both PSD-95 and PSD-93, respectively, whereas in the absence of PSD-93, results were undistinguishable from control. 
A

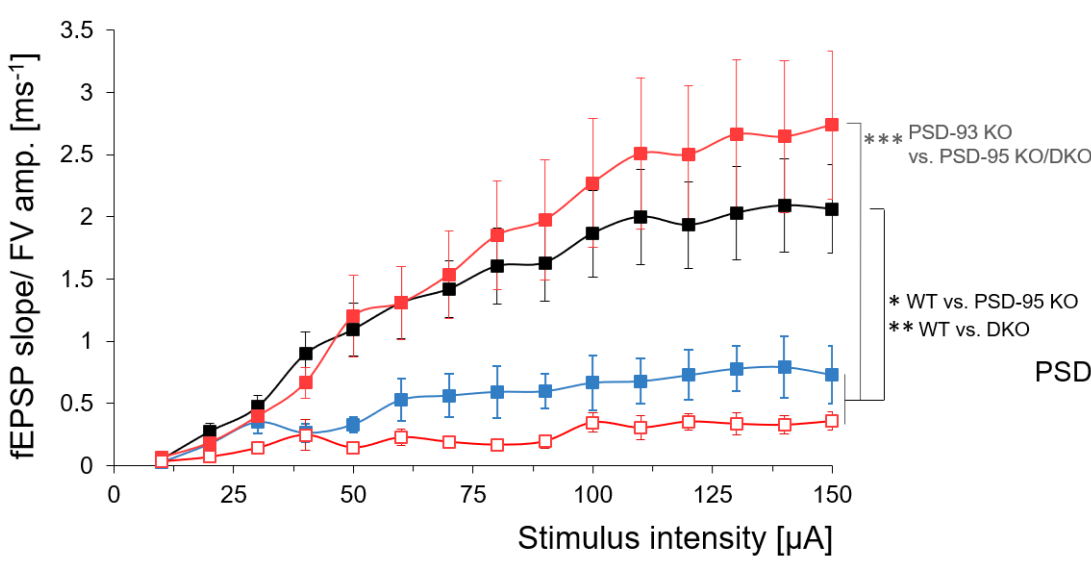

B

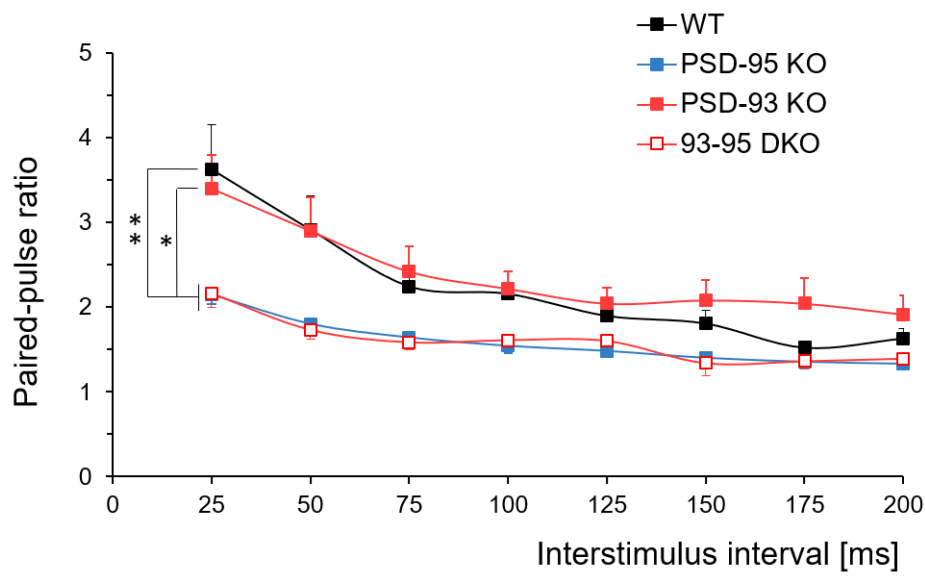

C
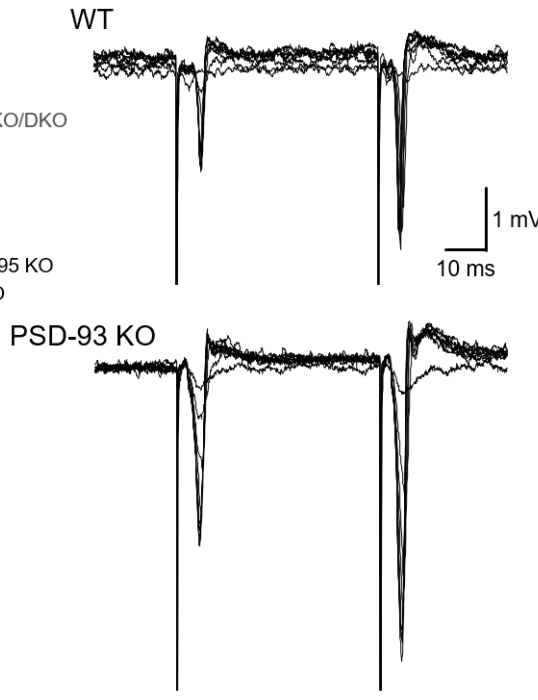

PSD-95 KO

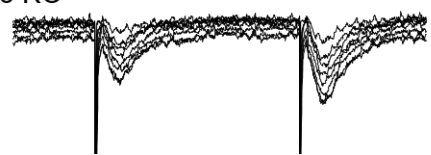

93-95 DKO

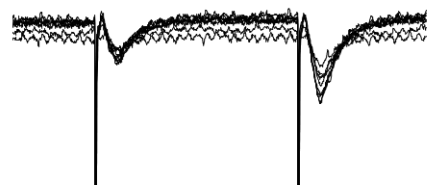

Figure 4: Changed hippocampal synaptic transmission and short-term synaptic plasticity in MAGUK KO mice. (A) The I-O curves showed decreased synaptic transmission in PSD-95 KO and PSD-93/95 DKO mice as compared to WT. In contrast, recordings from PSD-93 KO slices revealed significantly higher synaptic transmission than PSD-95 KO and PSD-93/95 DKO slices but were undistinguishable from WT mice. WT, $\mathrm{n} / \mathrm{m}=16 / 6$; PSD-95 KO, $\mathrm{n} / \mathrm{m}=15 / 7 ;$ PSD-93 KO, n/m $=15 / 4 ;$ PSD-93/95 DKO, $n / m=9 / 3$. (B) Positive PPR values (PPR, amplitude ratio EPSP2/EPSP1) indicated paired-pulse facilitation, which was decreased for PSD-95 KO and PSD-93/95 DKO mice. The PPR curve of PSD-93 KO samples was comparable to WT results. WT, $\mathrm{n} / \mathrm{m}=16 / 6$; PSD-95 $\mathrm{KO}, \mathrm{n} / \mathrm{m}=15 / 7$; PSD-93 KO, $\mathrm{n} / \mathrm{m}=16 / 4$; PSD-93/95 DKO, $\mathrm{n} / \mathrm{m}=10 / 3$. (C) Sample traces of fEPSP potential recordings with varying stimulus intensities $(10-150 \mu \mathrm{A})$. Traces are shown for an interstimulus interval of $50 \mathrm{~ms}$. (A-B) Two-way ANOVA followed by post-hoc LSD multiple comparison tests. ${ }^{* *} p<0.001 ;{ }^{* *} p<0.01 ;{ }^{*} p<0.05$. All data were given as means \pm SEM. 


\subsubsection{Electrical and optical properties of HSD waves}

To examine if the MAGUK KO results in modified susceptibility to hypoxia, I triggered HSD episodes by severe extracellular oxygen withdrawal in hippocampal slices. Characteristic SD waves were evoked by massive neuronal and glial depolarization, propagating throughout the brain tissue by synchronization of neighboring cells. This phenomenon is also optically visible and can be monitored through the so-called intrinsic optical signal (IOS) which provides a spatiotemporal pattern of the SD wave (Aitken et al., 1999; Andrew et al., 1999). The characteristic increase in tissue light reflectance starting at HSD onset is illustrated in Figure 5. The HSD wave predominantly invaded CA1 region, which is consistent with previous studies (Schmidt-Kastner \& Freund, 1991). Typically, it spread along the dentate gyrus and propagated through, but - if at all - just partly invaded the CA3 subfield. Granule cells of the dentate gyrus have been reported to be very resistant and just rarely show cell damage after relatively long ischemic periods (Schmidt-Kastner \& Hossmann, 1988). Nevertheless, the here seen invasion of the dentate gyrus is in accordance with other studies showing similar HSD wave patterns using acute mouse (Fischer et al., 2008) or rat brain slices (Basarsky et al., 1998). Notably, the reflectance increase was most pronounced in the dendritic layers (st. radiatum and st. oriens) but almost absent in st. pyramidale, in accordance with previous studies (Andrew et al., 1999).

A
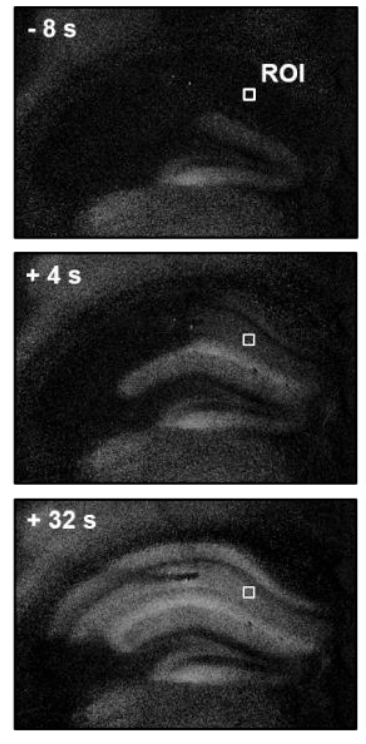

$1 \mathrm{~mm}$
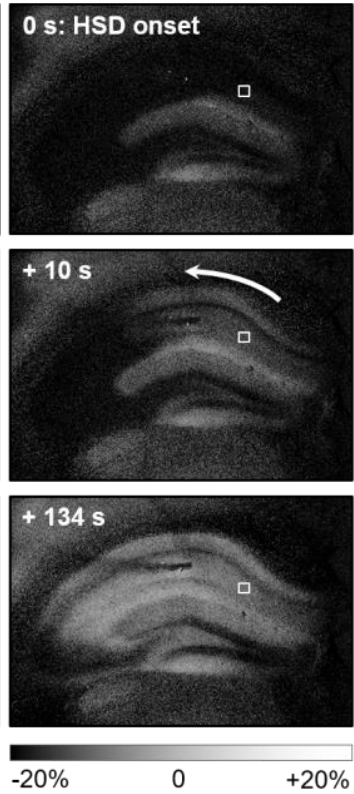

B

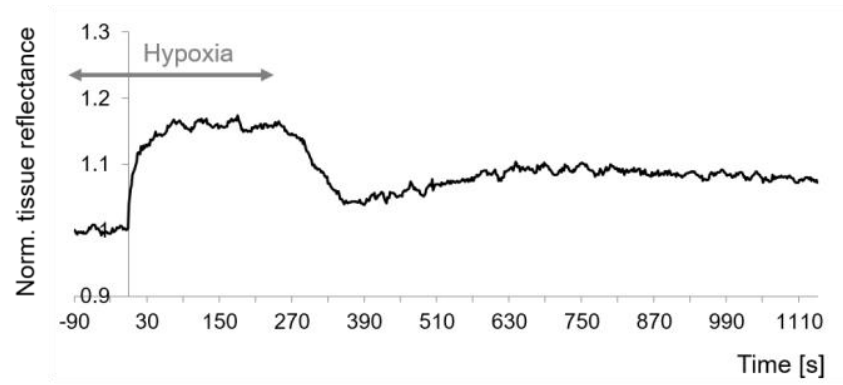

C

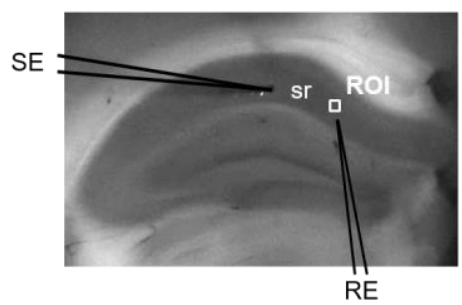

Figure 5: Intrinsic optical signal of the HSD wave in hippocampal slices. (A, B) The spatiotemporal IOS pattern shows an increase in tissue light reflectance starting at HSD onset $(\mathrm{t}=0)$. (A) Hippocampal tissue was illuminated with green light $(460-560 \mathrm{~nm})$ and HSD evoked following oxygen deprivation. Tissue light reflectance was detected in a small region of interest ( $\mathrm{ROI}$, indicated as a white square) in st. radiatum close to the recording electrode. Images result from subtraction as referred to normoxic baseline reflectance in order to visualize the changes in 
light reflectance due to HSD (256 gray-scale covering a total range of $\pm 20 \%$ brightness variation). The last image (+134 s) displays maximal spatial HSD spread. Time points relative to HSD onset at the recording electrode are provided in the left upper corner. (B) HSD-associated changes in tissue light reflectance normalized to prehypoxic baseline. In this example trace, HSD occurred $90 \mathrm{~s}$ after establishing a severe hypoxic environment. (C) Positions of the stimulation and recording electrode in the hippocampal slice. sr, stratum radiatum; SE, stimulation electrode; RE, recording electrode.

Moreover, HSD is accompanied by an abrupt, massive decrease in the direct current (DC) potential and therefore also electrophysiologically detectable (Leão, 1947; Müller \& Somjen, 2000a). Figure 6 displays this negative DC shift - which occurs 1-2 minutes after oxygen withdrawal - and its characteristic parameters. To test different severities of metabolic compromise, I varied the duration of the hypoxic state by initiating reoxygenation either from 1 minute, 2 minute or 4 minutes after HSD onset, with the latter being defined as the starting point of the DC shift. Longer time periods until reoxygenation resulted in later recovery of the DC potential and therefore in prolonged HSD durations (Figure $6 \mathrm{~A}-\mathrm{C}$ ). Each brain slice was exposed to hypoxic treatment only once to ensure similar health and neuronal activity conditions throughout all measurements. HSD was quantified using the following readouts: HSD amplitude (i.e. DC potential deflection), time to HSD onset, and duration of the half maximum amplitude (Figure 6D). 
A
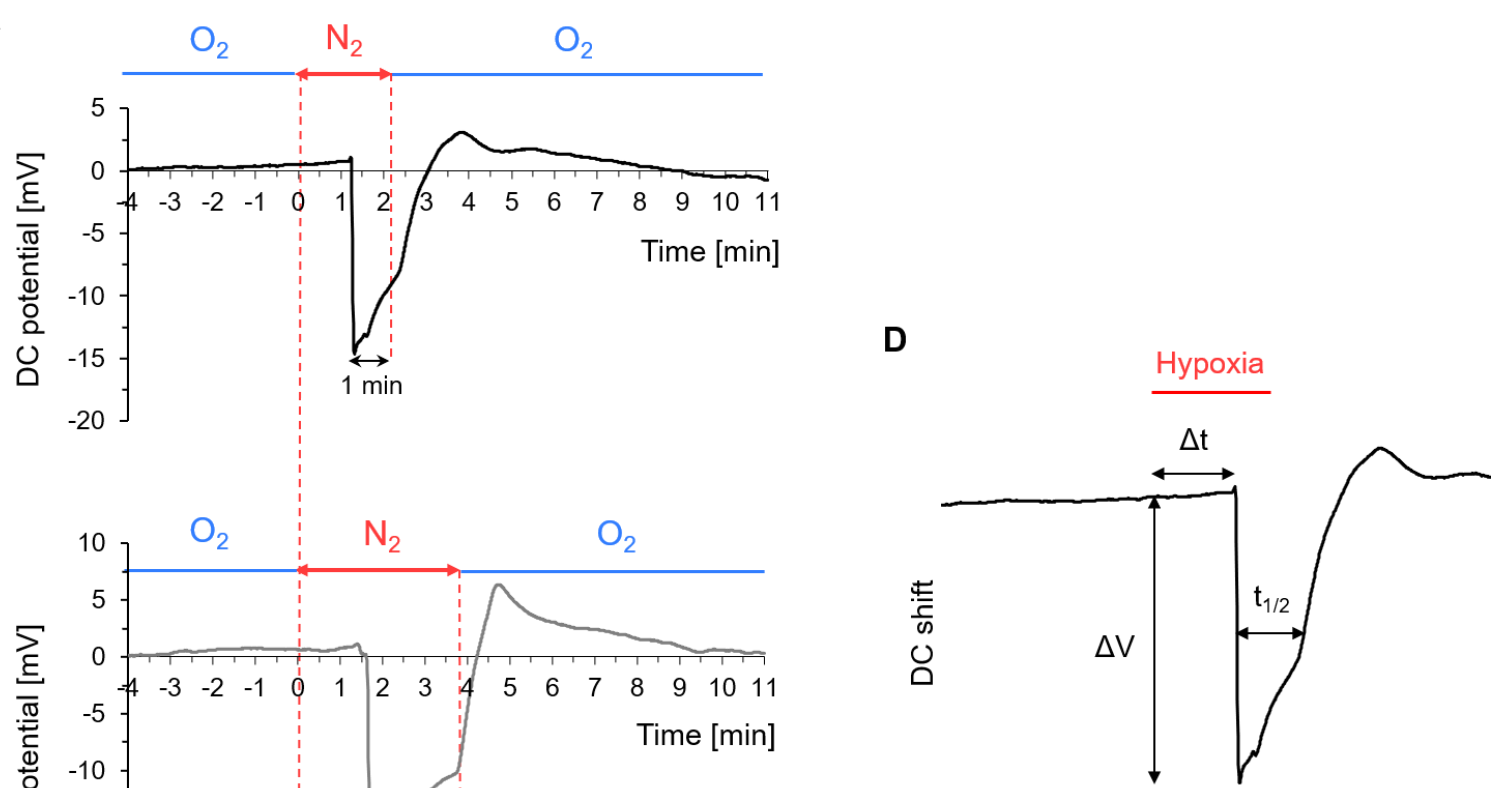

B

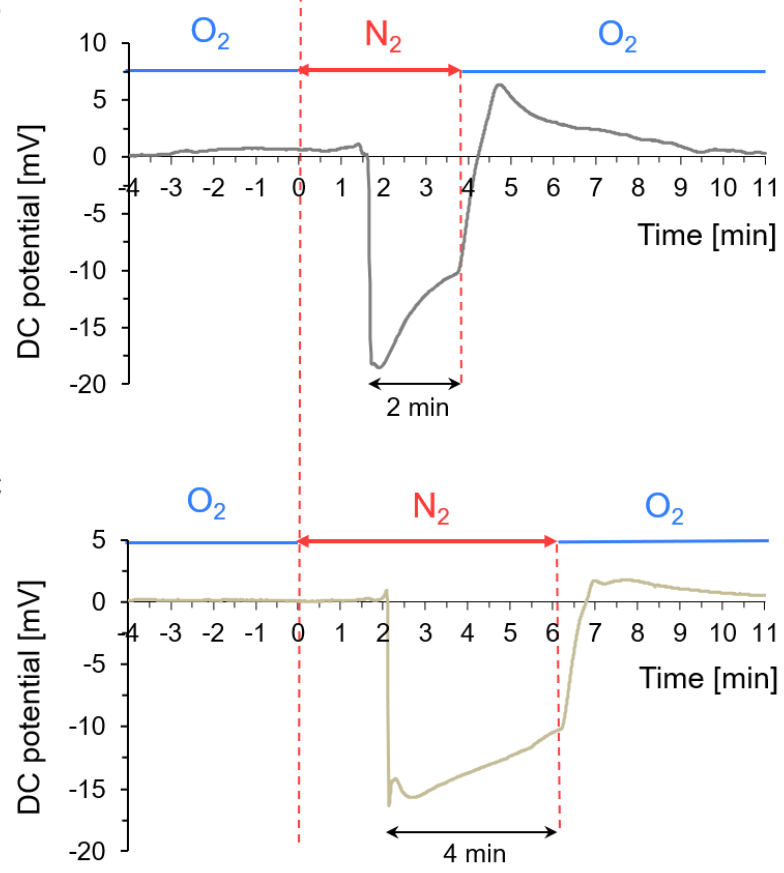

$\Delta V \quad H S D$ amplitude

$\Delta \mathrm{t}$ Time to HSD onset

$t_{1 / 2} \quad$ Duration at half maximum amplitude

Figure 6: HSD-accompanied negative deflection of the DC potential. (A-C) The initial graph sections show baseline extracellular field potential recordings under continuous carbogen $\left(95 \% \mathrm{O}_{2}\right.$ and $5 \% \mathrm{CO}_{2}$ ) supply, indicated as $\mathrm{O}_{2}$. At time point zero ( $\left.\mathrm{t}=0\right)$, gas supply was switched to nitrogen $\left(95 \% \mathrm{~N}_{2}\right.$ and $\left.5 \% \mathrm{CO}_{2}\right)$ for hypoxia induction, indicated as $\mathrm{N}_{2} .1-2$ min upon oxygen withdrawal, a rapid negative deflection of the DC potential marked the onset of HSD. 1 min (A), 2 min (B) or 4 min (C) after this onset, oxygen was resubmitted to the brain slice which allows the DC potential to recover. (D) Example trace of DC potential recordings illustrating characteristic parameters of HSD-associated negative deflection such as amplitude, time to onset and the duration measured at half maximum amplitude. 


\subsubsection{Delayed onset of hypoxia-induced spreading depression in PSD-95 KO mice}

In the following, I analyzed HSD in WT and KO mice at 2 months of age (P55-65) and found that the onset upon oxygen withdrawal was strongly dependent on genotype [Figure 7A; $F_{3,184}$ $=5.787, p=0.001]$. Interestingly, HSD onset was delayed by $\sim 20 \%$ in PSD-95 KO slices as compared to control [WT: $106.20 \pm 3.43$ s; PSD-95 KO: $126.85 \pm 6.25$ s; PSD-95 KO vs. WT, $\mathrm{p}=0.002$ ]. Moreover, PSD-95 KO mice exhibited later HSD onset compared to PSD-93 KO and PSD-93/95 DKO, both of which were undistinguishable from WT [PSD-93 KO: $113.81 \pm$ 4.04 s; PSD-93/95 DKO: $101.79 \pm 3.80$ s; PSD-95 KO vs. PSD-93 KO, $p=0.041$; PSD-95 KO vs. PSD-93/95 DKO, $p<0.001$; all other $p$ values $>0.05$ ]. Together, these results indicate reduced susceptibility to hypoxia in the absence of PSD-95 but not PSD-93. Notably, simultaneous deletion of PSD-93 counteracted the protective effects of PSD-95 KO, as evidenced by normal HSD onset in DKO slices.

The DC potential drop in WT samples reached an amplitude of $-17.14 \pm 0.40 \mathrm{mV}$, which turned out to be not affected by genotype [Figure 7B; PSD-95 KO: $-16.59 \pm 0.45 \mathrm{mV}$; PSD-93 KO: $-16.97 \pm 0.40 \mathrm{mV}$; PSD-93/95 DKO: $\left.-16.92 \pm 0.34 \mathrm{mV} ; F_{3,184}=0.327, p=0.806\right]$.

I obtained similar results for the HSD duration, which was unchanged in the absence of either PSD-93 or PSD-95 and both proteins simultaneously (Figure 7C). The HSD durations for the three different time periods until reoxygenation were analyzed separately [ $1 \mathrm{~min}: F_{3,64}=0.953$, $\left.p=0.420 ; 2 \min : F_{3,59}=0.670, p=0.574 ; 4 \min : F_{3,53}=0.803, p=0.498\right]$. For early reoxygenation (after $1 \mathrm{~min}$ ), HSD lasted $82.36 \pm 2.76 \mathrm{~s}$ in WT hippocampi [PSD-95 KO: 93.10 $\pm 10.13 \mathrm{~s}$; PSD-93 KO: $89.06 \pm 3.27 \mathrm{~s}$; PSD-93/95 DKO: $88.80 \pm 2.58 \mathrm{~s}$ ] and increased by an average of $\sim 64 \%$ to $134.86 \pm 4.47 \mathrm{~s}$ if the hypoxic phase was doubled in time [reoxygenation 2 min after HSD onset: PSD-95 KO: $138.80 \pm 1.93 \mathrm{~s}$; PSD-93 KO: $138.31 \pm 1.70 \mathrm{~s}$; PSD93/95 DKO: $140.04 \pm 1.77 \mathrm{~s}$ ]. By again doubling the time for reoxygenation to 4 minutes, the HSD duration was lengthened by about $53 \%$ to $206.78 \pm 17.68$ s [PSD-95 KO: $212.07 \pm$ 15.96 s; PSD-93 KO: $213.60 \pm 14.47$ s; PSD-93/95 DKO: $235.96 \pm 9.83$ s].

The profile of negative DC deflection mostly showed the characteristic inverted saddle shape, comprised of an early sharp peak followed by a second negative peak or lower plateau (Figure 7D), with the latter reported to be mainly shaped by NMDAR-mediated currents (Marrannes et al., 1988; Herreras \& Somjen, 1993; Krüger et al., 1999).

HSD-induced DC potential changes are typically divided into three phases (Martin et al., 1994; Frenguelli, 1997). The initial short hyperpolarization is thought to result from enhanced membrane conductance to $\mathrm{K}^{+}$via the activation of voltage-gated, ATP- and/or $\mathrm{Ca}^{2+}$-sensitive $\mathrm{K}^{+}$channels. Subsequently, a rapid and marked depolarization (second phase) is mediated by 
GluR currents and the inhibition of $\mathrm{Na}^{+} / \mathrm{K}^{+}-\mathrm{ATPase}$, leading to disrupted ion homeostasis and near complete depolarization of neurons and glial cells. After reoxygenation, extracellular ion concentrations and neurotransmitter levels become reinstated mainly by the activity of $\mathrm{Na}^{+} / \mathrm{K}^{+}-$ ATPase and glutamate uptake (Martin et al., 1994). Typically, these processes transiently overshoot as seen by a posthypoxic hyperpolarization with a DC potential higher than baseline levels (Figure 7D) (Hansen \& Zeuthen, 1981).

A

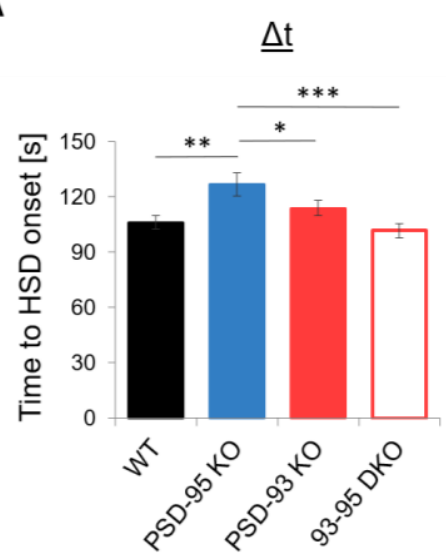

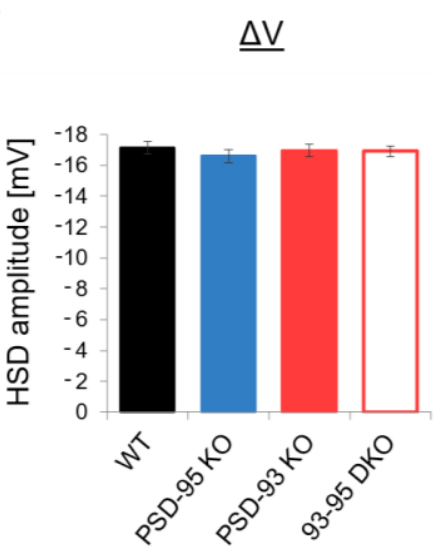

C

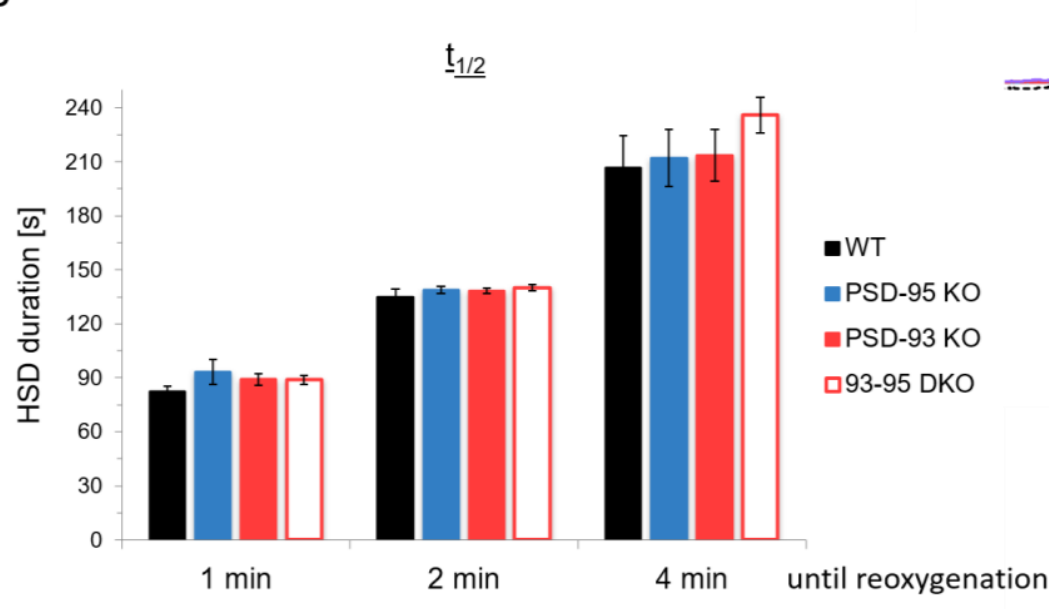

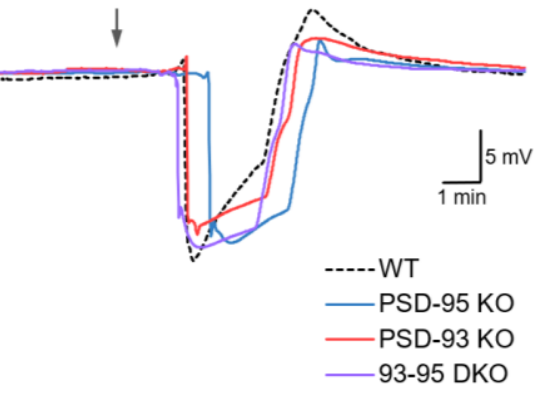

Figure 7: Time to HSD onset was prolonged by loss of PSD-95. (A) Bar graph depicting the times to HSD onset following oxygen withdrawal. PSD-95 KO slices showed delayed HSD onset compared to WT, PSD-93 KO and PSD-93/95 DKO mice. (B) No significant changes were observed for the amplitude of negative DC potential deflection. (C) Bar graph displaying HSD duration which showed no genotype effect, irrespective of the reoxygenation condition (1 min, $2 \mathrm{~min}$ and $4 \mathrm{~min}$, respectively). (D) Sample traces of DC potential recordings with the arrow indicating gas supply switch from oxygen to nitrogen for hypoxia induction. In this sample trace, slices were reoxygenated 2 min after the onset of HSD. (A, B) WT, n/m = 44/9; PSD-95 KO, n/m = 48/12; PSD-93 KO, $\mathrm{n} / \mathrm{m}=50 / 11$; PSD-93/95 DKO, $\mathrm{n} / \mathrm{m}=46 / 11$. (C) $1 \mathrm{~min}: \mathrm{WT}, \mathrm{n} / \mathrm{m}=16 / 9$; PSD$95 \mathrm{KO}, \mathrm{n} / \mathrm{m}=19 / 11$; PSD-93 KO, $\mathrm{n} / \mathrm{m}=17 / 9$; PSD-93/95 DKO, $\mathrm{n} / \mathrm{m}=16 / 11.2 \mathrm{~min}: \mathrm{WT}, \mathrm{n} / \mathrm{m}=$ 15/9; PSD-95 KO, $\mathrm{n} / \mathrm{m}=15 / 10$; PSD-93 KO, $\mathrm{n} / \mathrm{m}=18 / 11$; PSD-93/95 DKO, $\mathrm{n} / \mathrm{m}=15 / 9.4 \mathrm{~min}$ : WT, $\mathrm{n} / \mathrm{m}=13 / 9 ;$ PSD-95 KO, $\mathrm{n} / \mathrm{m}=14 / 10$; PSD-93 KO, $\mathrm{n} / \mathrm{m}=15 / 8$; PSD-93/95 DKO, $\mathrm{n} / \mathrm{m}=15 / 9$. Oneway ANOVA followed by post-hoc LSD multiple comparison tests. ${ }^{* \star *} p<0.001$; ${ }^{* *} p<0.01$; ${ }^{*} p<$ 0.05 . All data were given as means \pm SEM. 


\subsubsection{Modified intrinsic optical signals indicate a protective capacity of PSD-95 loss against hypoxia}

\subsubsection{Loss of PSD-95 attenuates the increase of tissue light reflectance during $\mathrm{HSD}$}

The protective effect of PSD-95 KO against hypoxia, as so far indicated by delayed HSD onset, was also observed on the level of HSD-accompanying change in tissue light reflectance (IOS). The IOS displayed a characteristic profile with a brief, weak decrease in tissue light reflectance (visible in Figure $8 \mathrm{~A}$ and $\mathrm{C}$ ), followed by a sharp and pronounced increase, which slowly declined after resubmission of oxygen. Frequently, a secondary increase during recovery was visible, which was likely caused by "undershoot" of the cell volume as cells recover from the preceding swelling (Figure 8, arrowheads) (Andrew et al., 1999; Somjen, 2001). Furthermore, dendritic beading has been shown to occur in the brain following ischemia and might as well account for the increase in light scattering (Hori \& Carpenter, 1994).

As seen in Figure 8A and $\mathrm{B}$, the increase in reflectance was markedly attenuated in PSD-95 KO mice when oxygen was resupplied 1 or 2 minutes upon HSD onset. The same tendency was present for longer hypoxic periods (4 min, Figure $8 \mathrm{C}$ ). Furthermore, PSD-93/95 DKO slices showed a somewhat lower reflectance change for early reoxygenation (1 minute) and a slowly developing, moderate secondary increase during recovery, which was absent in the other experimental groups. In contrast to affected intensities of tissue reflectance change, the time course seemed to be unaltered among the genotypes.

In detail, by comparing the maximal tissue reflection (Figure 8D), control slices demonstrated $20.40 \pm 1.58 \%$ increase upon hypoxia for the 1 minute condition, whereas this signal was substantially reduced to $11.23 \pm 1.52 \%$ in the absence of PSD-95 $\left[F_{3,55}=7.617, p<0.001\right.$; PSD-95 KO vs. WT, $p<0.001]$. The KO of PSD-93 as well as PSD-93 and PSD-95 simultaneously did not have significant effects as compared to WT; however, reflectance increases were stronger than in PSD-95 KO mice [PSD-93 KO: $19.52 \pm 1.51 \%$; PSD-93/95 DKO: $16.46 \pm 1.46 \%$; PSD-95 KO vs. PSD-93 KO, $p<0.001$; PSD-95 KO vs. PSD-93/95 DKO, $\mathrm{p}=0.016]$.

I obtained similar results for IOS intensities when the brain tissue was reoxygenated 2 minutes after HSD onset. WT hippocampi showed an increase of $19.68 \pm 1.33 \%$, whereas light reflectance augmented by only $14.82 \pm 1.65 \%$ in PSD-95 KO mice [PSD-93 KO: $20.82 \pm$ 1.39\%; PSD-93/95 DKO: $20.01 \pm 1.27 \%$; $F_{3,58}=3.720, p=0.016$; PSD-95 KO vs. WT, $p=$ 0.020; PSD-95 KO vs. PSD-93 KO, $p=0.003$; PSD-95 KO vs. PSD-93/95 DKO, $p=0.015$ ]. 
Regarding the immense prolonged and stressful hypoxic period up to 4 minutes, no genotype differences were evident [WT: $18.47 \pm 1.40 \%$; PSD-95 KO: $16.74 \pm 1.84 \%$; PSD-93 KO: $21.23 \pm 1.72 \%$; PSD-93/95 DKO: $\left.21.21 \pm 1.65 \% ; F_{3,48}=1.755, p=0.168\right]$. Nevertheless, tissue light reflectance recovered much slower and remained quite high after reoxygenation, especially in case of PSD-93 KO and PSD-93/95 DKO mice. Strikingly, the second increase was intensified and prolonged in all slices, irrespective of genotype (Figure $8 \mathrm{C}$ ). All these observations point to a diminished or even absent recovery of neuronal tissue after the extensive time period of oxygen lack. This aspect, I will further examine in section 3.1.5.3 by assessing synaptic recovery via recording of evoked responses.

In conclusion, when slices were reoxygenated within less than 4 minutes, the HSD-associated increase in tissue light reflectance was strongly attenuated in the absence of PSD-95. This effect was seen in slices reoxygenated either 1 or 2 minutes after HSD onset and thus replicable. In line with my previous findings on HSD onset, these data point to a protective effect of the PSD-95 loss against hypoxia-induced network dysfunction. Strikingly, this effect was abolished by simultaneous deletion of PSD-93.

[figure on next page]

Figure 8: Reduced light reflectance response of hippocampal tissue in PSD-95 KO mice after HSD induction. (A-C) Temporal pattern of the reflectance changes aligned to the onset of HSD (at $\mathrm{t}=0$ ). Oxygen was resupplied $1 \mathrm{~min}(\mathbf{A}), 2 \mathrm{~min}$ (B) and $4 \mathrm{~min}$ (C) after HSD onset, respectively. Arrowheads indicate a secondary increase in tissue light reflectance. (D) Bar graph depicting the corresponding maximal tissue reflectance rise shown in A-C. PSD-95 KO mice displayed reduced intensity changes as compared to all other genotypes in case of reoxygenation 1 min or 2 min upon HSD onset. Diminished recovery was observed in all brain slices facing the longest hypoxic time period (4 min, C). $1 \mathrm{~min}: \mathrm{WT}, \mathrm{n} / \mathrm{m}=13 / 9$; PSD-95 KO, $\mathrm{n} / \mathrm{m}=16 / 10$, PSD-93 $K O, n / m=15 / 10 ;$ PSD-93/95 DKO, $n / m=15 / 10.2 \mathrm{~min}: \mathrm{WT}, \mathrm{n} / \mathrm{m}=15 / 9 ;$ PSD-95 KO, $\mathrm{n} / \mathrm{m}=16 / 10$; PSD-93 KO, $n / m=17 / 11$; PSD-93/95 DKO, $n / m=14 / 10.4$ min: WT, $n / m=15 / 9 ; P S D-95 ~ K O, n / m$ $=16 / 11 ; \mathrm{PSD}-93 \mathrm{KO}, \mathrm{n} / \mathrm{m}=17 / 9 ; \mathrm{PSD}-93 / 95 \mathrm{DKO}, \mathrm{n} / \mathrm{m}=14 / 10$. One-way ANOVA followed by post-hoc LSD multiple comparison tests. ${ }^{* * *} p<0.001 ;{ }^{* *} p<0.01 ;{ }^{*} p<0.05$. All data were given as means \pm SEM. 
A

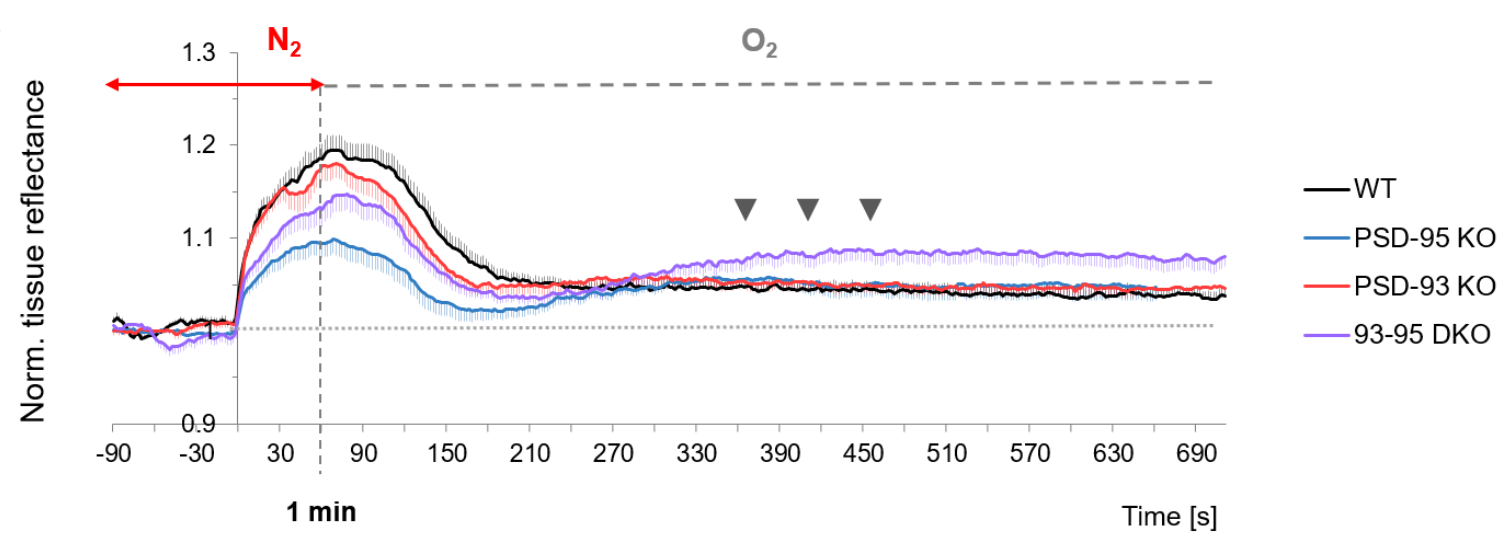

B

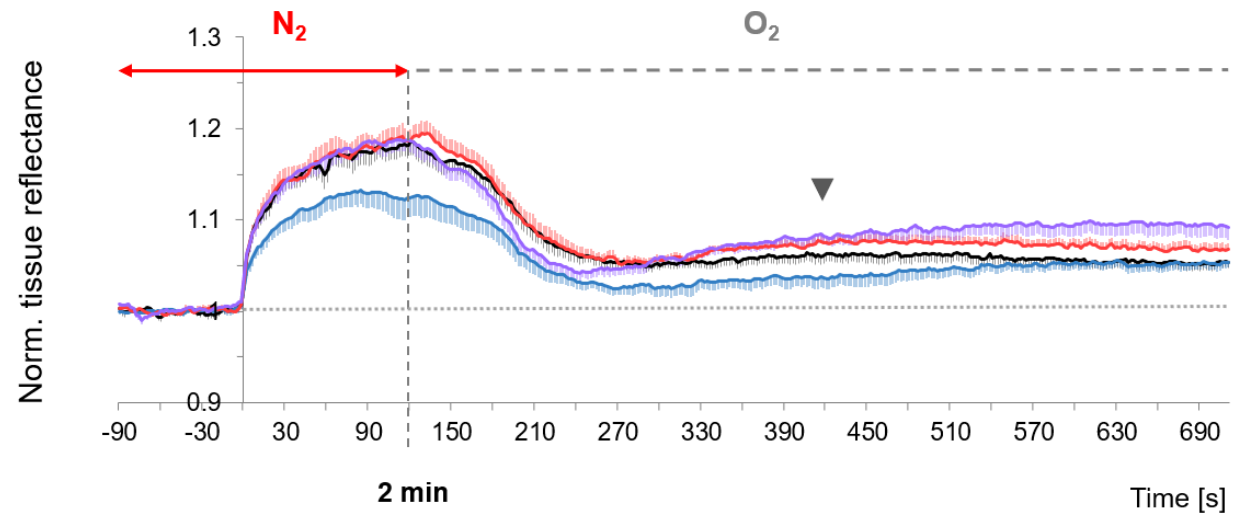

C

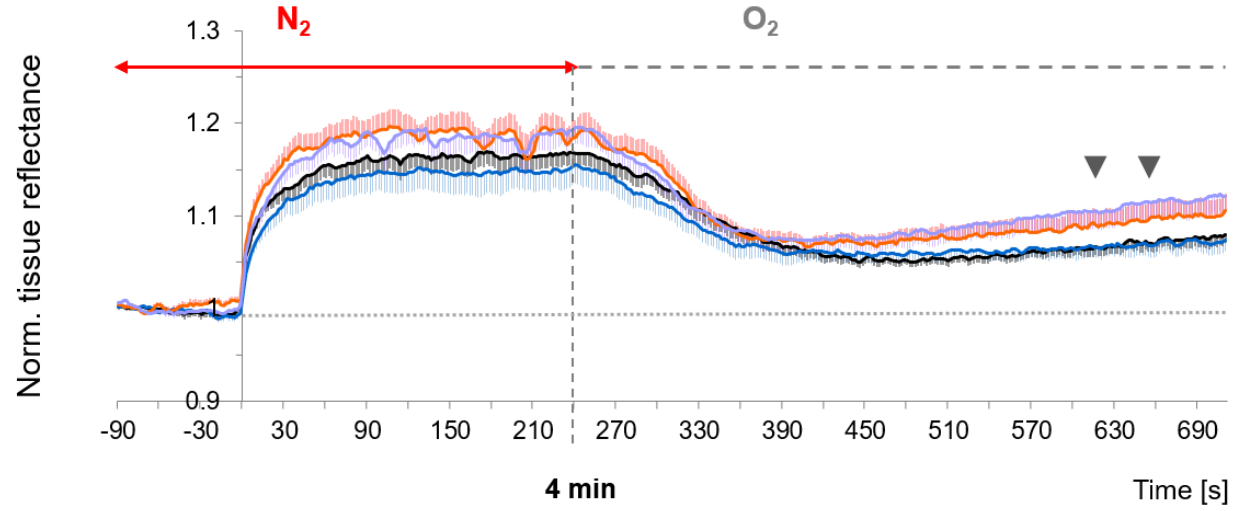

D

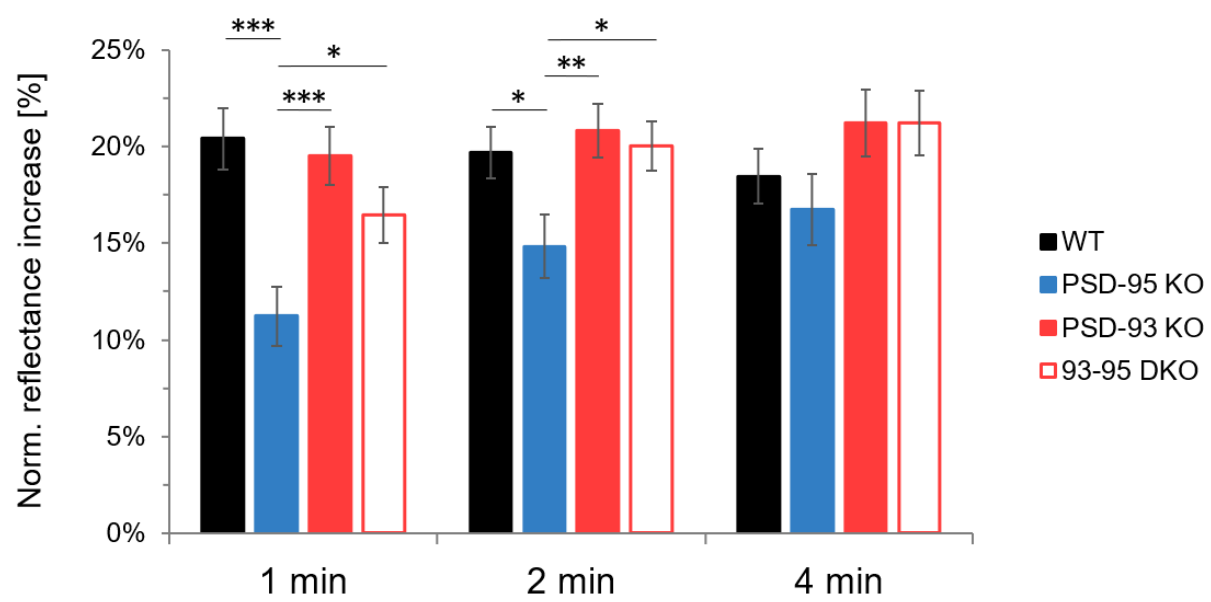




\subsubsection{Trend to decelerated HSD wave propagation in PSD-95 KO slices}

Further analysis of the IOS provided information about HSD wave characteristics such as its propagation velocity and maximum spread throughout the brain slice.

At light reflectance peak, the relative tissue area being invaded by an HSD episode was calculated with reference to total hippocampal size. In WT mice, $67.92 \pm 0.013 \%$ of the hippocampus displayed IOS effects, which did not differ significantly from those areas obtained in KO mice [Figure 9A, PSD-95 KO: $64.48 \pm$ 0.017\%; PSD-93 KO: $65.88 \pm 0.017 \%$; 93-95 DKO: $\left.65.42 \pm 0.014 \% ; F_{3,170}=0.864, p=0.461\right]$. Therefore, PSD-95 loss could not protect parts of the hippocampus from the hypoxic, depolarized state by supposably impaired synchronization of neighboring neurons, which could prevent further HSD wave propagation.

In contrast, a trend towards a genotype effect was gained from the analyzed velocities of HSD wave propagation [Figure 9B; $F_{3,175}=2.508, p=0.061$ ]. Even though the main effect was not significant, I did an exploratory comparison among the genotypes. In control samples, the velocity averaged in $6.62 \pm 0.27 \mathrm{~mm} / \mathrm{min}$, which was decelerated to $5.72 \pm 0.26 \mathrm{~mm} / \mathrm{min}$ in the absence of PSD-95, corresponding to a decrease of $\sim 13.6 \%$ [PSD-95 KO vs. WT, $p=$ 0.036]. Again, propagation velocities of PSD-93 KO as well as PSD-93/95 DKO were similar to WT levels, but higher compared to PSD-95 KO [PSD-93 KO: $6.57 \pm 0.28 \mathrm{~mm} / \mathrm{min}$; PSD-93/95 DKO: $6.72 \pm 0.22 \mathrm{~mm} / \mathrm{min}$; PSD-95 KO vs. PSD-93 KO, $\mathrm{p}=0.039$; PSD-95 KO vs. PSD$93 / 95$ DKO, $p=0.017$; all other $p$ values $>0.05]$. These observations corroborate my previous results showing partly dampened hypoxia-induced impairments in PSD-95 KO neuronal networks. Specifically, in this case, by a decelerated transfer of the depolarized and nonfunctional neuronal state onto the neighboring tissue. The effect of PSD-95 loss was again abrogated by simultaneous knockout of PSD-93. 
A

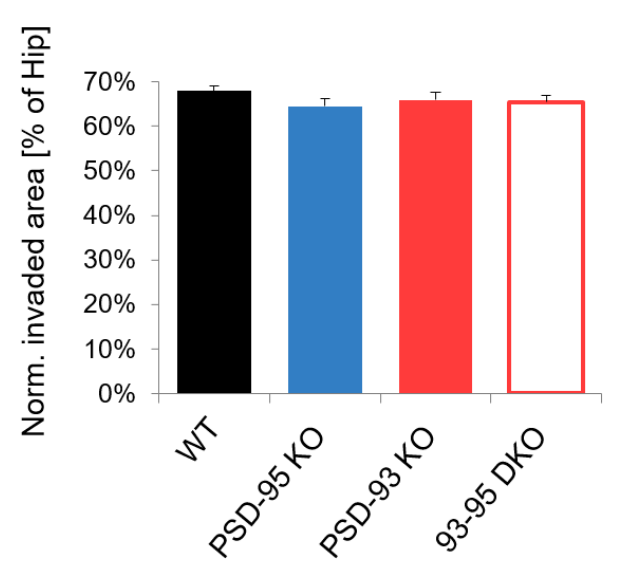

B

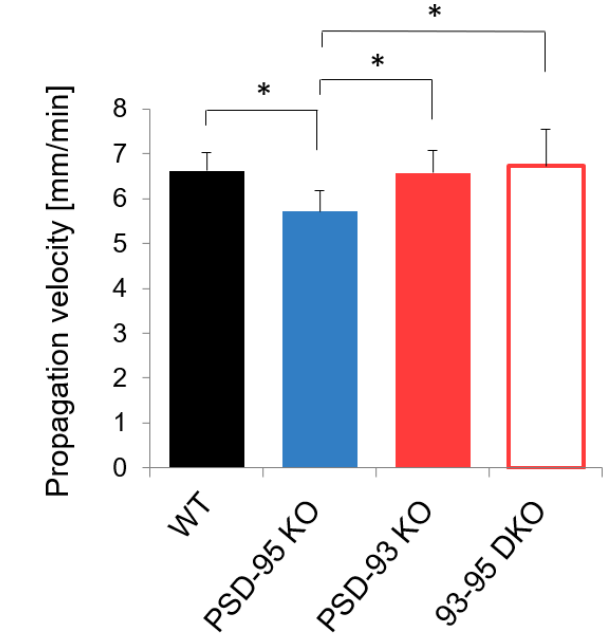

Figure 9: The HSD wave proliferated more slowly in the absence of PSD-95. (A) Bar graph illustrating the HSD invaded area as referred to total hippocampus, which was undistinguishable among genotypes. WT, $\mathrm{n} / \mathrm{m}=41 / 9$; PSD-95 KO, $\mathrm{n} / \mathrm{m}=46 / 12$; PSD-93 $\mathrm{KO}, \mathrm{n} / \mathrm{m}=44 / 11$; 93-95 DKO, $n / m=43 / 11$. (B) Propagation of the HSD wave front detected in st. radiatum parallel to st. pyramidale was diminished due to loss of PSD-95 compared to all other genotypes. WT, $\mathrm{n} / \mathrm{m}=$ 41/9; PSD-95 KO, $\mathrm{n} / \mathrm{m}=46 / 12$; PSD-93 KO, $\mathrm{n} / \mathrm{m}=45 / 11$; PSD-93/95 DKO, $\mathrm{n} / \mathrm{m}=43 / 11$. (A, B) One-way ANOVA followed by post-hoc LSD multiple comparison tests. ${ }^{*} p<0.05$. All data were given as means \pm SEM.

\subsubsection{Partly reduced hypoxia-induced synaptic dysfunction in PSD-95 KO and PSD-93/95 DKO mice}

Throughout optical and electrophysiological monitoring of hypoxia-treated brain slices, I evoked fEPSPs to visualize synaptic function of the neuronal network. To this end, I stimulated the SC-CA1 hippocampal pathway every $20 \mathrm{~s}$ with an intensity of $100 \mu \mathrm{A}$ and analyzed changes in resulting fEPSP amplitudes.

\subsubsection{Reduced excitability of PSD-95 KO and PSD-93/95 DKO neuronal networks}

By screening fEPSPs of the normoxic baseline, amplitudes were reduced to approximately $50 \%$ in PSD-95 KO and PSD-93/95 DKO mice as compared to control and as well PSD-93 KO slices (Figure 10) [WT: $100.00 \pm$ 0.05\%; PSD-95 KO: $52.38 \pm 0.03 \%$; PSD-93 KO: $111.37 \pm$ 0.07\%; 93-95 DKO: $42.86 \pm 0.02 \% ; F_{3,184}=53.246, p<0.001 ; P S D-95 \mathrm{KO}:$ vs. WT, $p<0.001$, vs. PSD-93 KO, $p<0.001$, vs. PSD-93/95 DKO, $p=0.150$; PSD-93 KO: vs. PSD93/95 DKO, $p<0.001$, vs. WT, $p=0.090$; PSD-93/95 DKO vs. WT, $p<0.001]$. This is in line with the genotype differences seen for the initial I-O curve measurements (3.1.1 and Figure 4) and indicates reduced basal synaptic transmission in PSD-95 KO and DKO mice. 
A

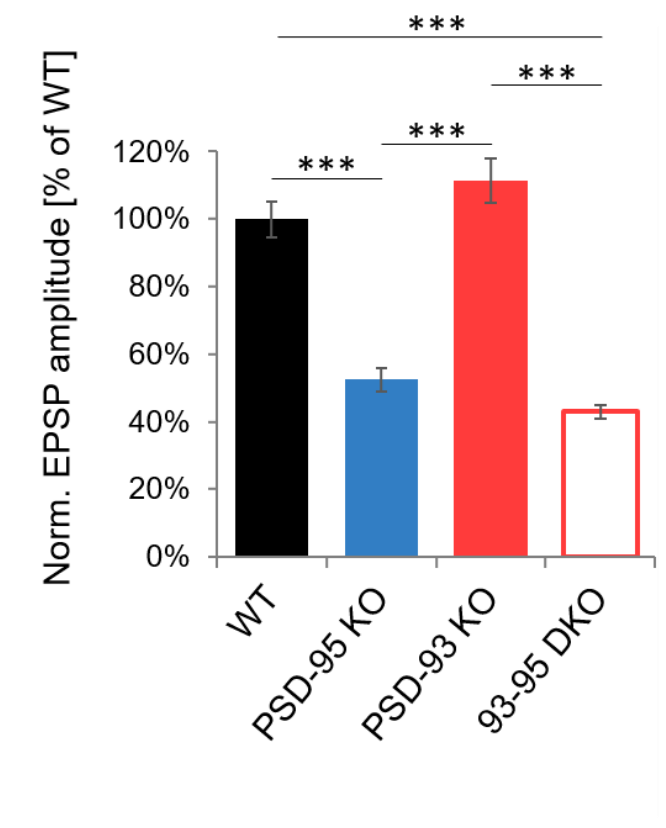

B
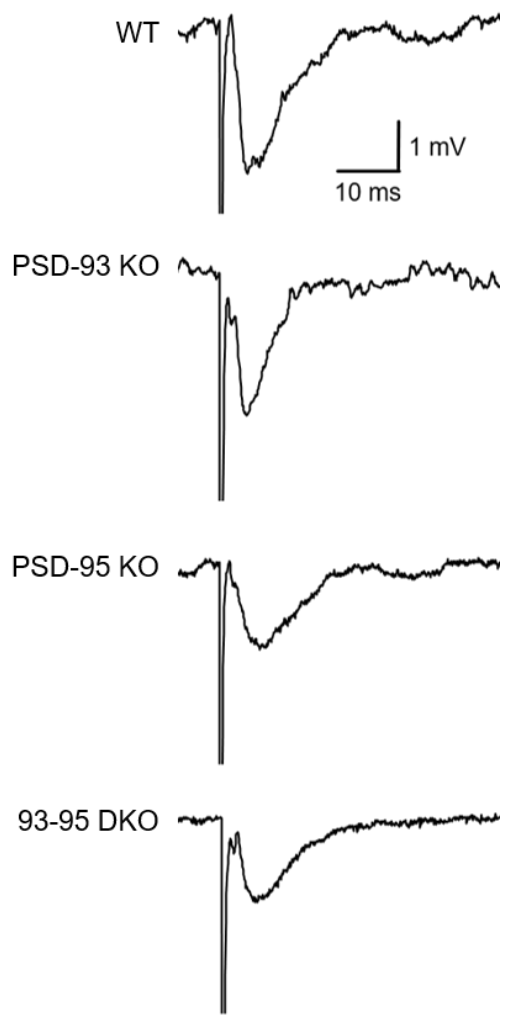

Figure 10: PSD-95 KO and PSD-93/95 DKO fEPSP amplitudes showed 50\% reduction. As part of DC potential recordings, hippocampal networks were stimulated every $20 \mathrm{~s}$ with an intensity of $100 \mu \mathrm{A}$. (A) The evoked fEPSP amplitudes are normalized to WT levels. In PSD-95 KO and PSD-93/95 DKO slices, fEPSP amplitudes were reduced to about 50\% compared to control and PSD-93 KO. WT, $\mathrm{n} / \mathrm{m}=44 / 9$; PSD-95 KO, $\mathrm{n} / \mathrm{m}=48 / 12$; PSD-93 KO, $\mathrm{n} / \mathrm{m}=49 / 11 ; 93-95 \mathrm{DKO}$, $\mathrm{n} / \mathrm{m}=46 / 11$. One-way ANOVA followed by post-hoc LSD multiple comparison tests. ${ }^{* * *} \mathrm{p}<0.001$. All data were given as means \pm SEM. (B) Sample traces of fEPSP potential recordings with a stimulus intensity of $100 \mu \mathrm{A}$. 


\subsubsection{Loss of synaptic function in acute brain slices due to oxygen deprivation}

The effects of hypoxia on synaptic function of the SC-CA1 hippocampal pathway are demonstrated in Figure 11. Approximately $40 \mathrm{~s}$ upon oxygen withdrawal, fEPSP amplitudes decreased within about $20 \mathrm{~s}$ to $50 \%$ as referred to normoxic baseline conditions. After $\sim 2$ minutes following oxygen deprivation, total loss of synaptic function was indicated by the absence of fEPSP responses as seen in the recording traces. Following semi-automated analysis, amplitudes seem to remain $10-30 \%$ of their intensity due to noise peak detection. As baseline fEPSP amplitudes were about 50\% decreased in PSD-95 KO and PSD-93/95 DKO mice (Figure 10), the noise effect was enhanced when normalized to baseline and therefore misleads to reduced synaptic loss compared to WT and PSD-93 KO. Thus, statistical analysis was neglected. On the descriptive level, I detected similar time courses for the experimental mouse groups of reoxygenation 1 minute, 2 minutes and 4 minutes after the onset of HSD. However, the loss of synaptic function seemed to be slightly delayed in PSD-95 KO mice, at least for the conditions where slices were reoxygenated after 1 minute and 4 minutes, respectively (Figure $11 \mathrm{~A}$ and $\mathrm{C}$, indicated by arrowheads).

The early occurring synaptic failure represents a major difference of the HSD observed here, to normoxic SD (Somjen, 2001). While in normoxic SD, synapses continue to function until their ion channels get inactivated due to depolarization, synaptic failure in the present study was already seen around one minute before the actual HSD onset. Indeed, the earliest consequence of cerebral ischemia is the loss of synaptic activity (Hofmeijer et al., 2012). There are various mechanisms which might contribute to the early synaptic failure during hypoxia and can occur independently of the following depolarization-induced SD (Somjen et al., 1993). Most studies indicate presynaptic dysfunction such as impaired neurotransmitter release (Crépel et al., 1993; Frenguelli, 1997; Sun et al., 2002), most likely due to disturbed activity of voltage-dependent $\mathrm{Ca}^{2+}$ channels at the presynaptic terminal (Adams et al., 1985; Ouanonou et al., 1999). On the other hand, evidence for postsynaptic dysfunction was provided by enhanced $\mathrm{K}^{+}$conductance of the postsynaptic membrane, in turn contributing to diminished excitatory transmission by raising the firing threshold (Somjen et al., 1993). Additionally, increased intracellular $\mathrm{Ca}^{2+}$ levels - similar to the presynapse - might arise from NMDAR overactivation and release from intracellular stores (Nowicky \& Duchen, 1998), hence disturbing physiological ion gradients and contributing to synaptic failure as seen here. 
A

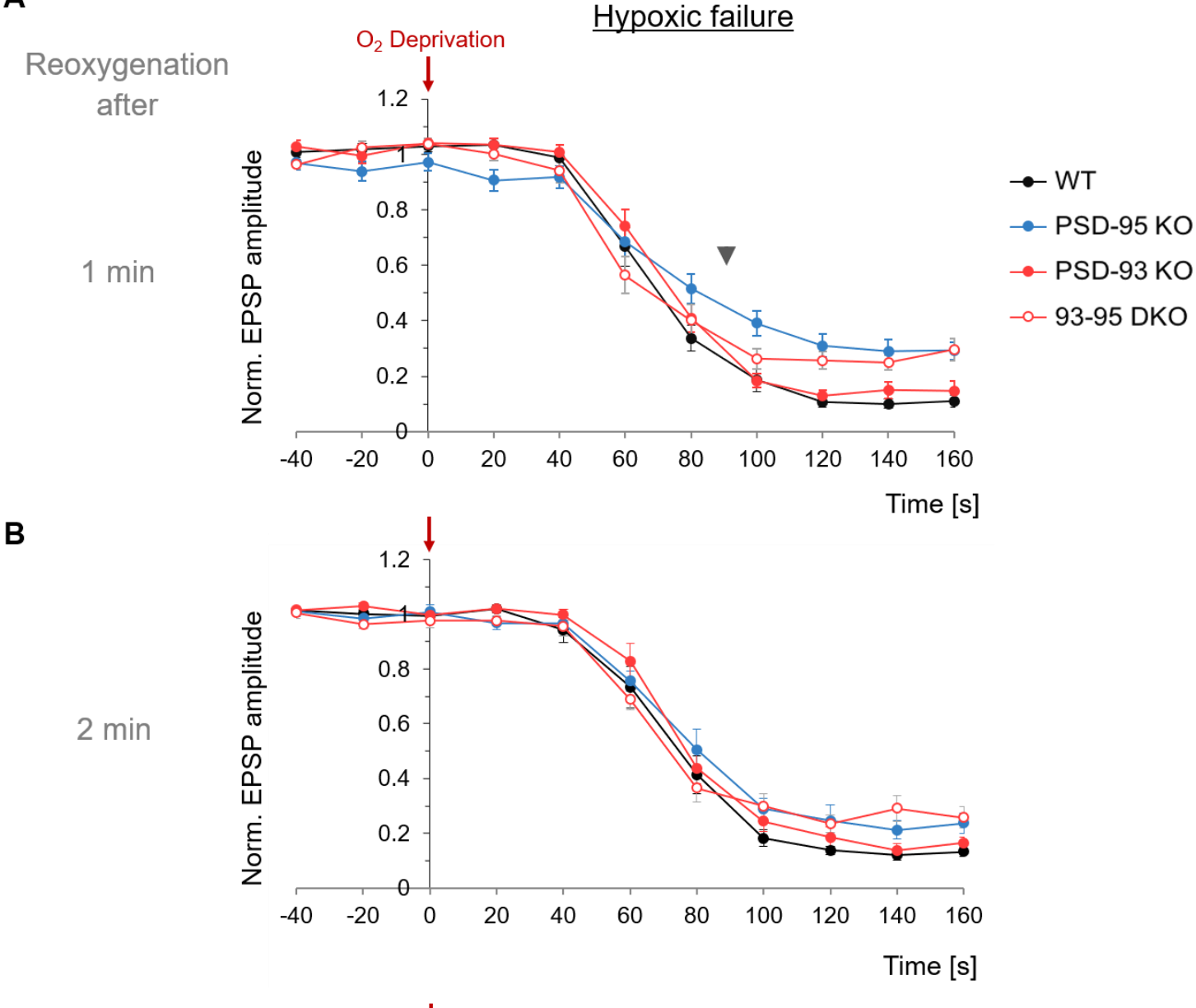

C

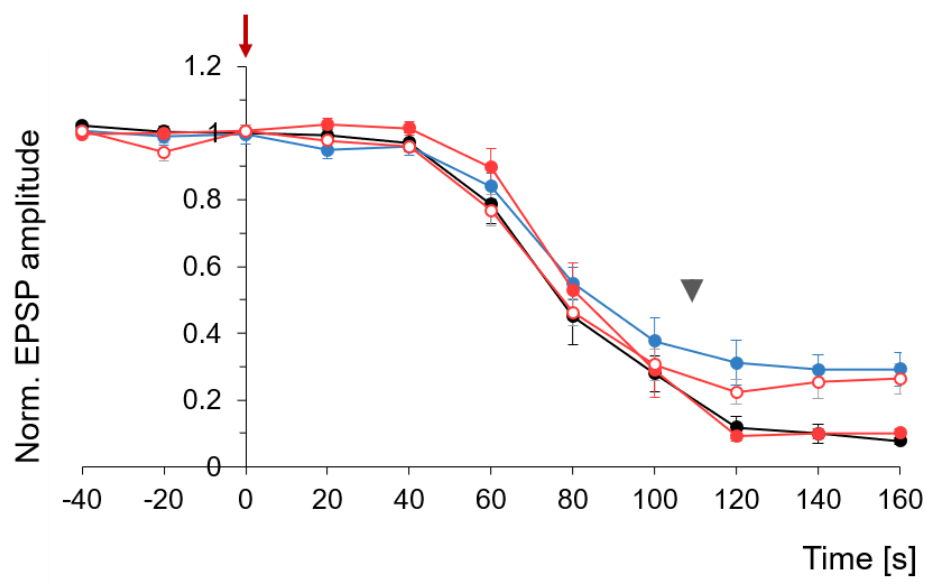

Figure 11: Hypoxia-induced loss of synaptic function in acute hippocampal slices. (A-C) Line graphs depicting fEPSP amplitudes normalized to normoxic baseline conditions. Datasets for $1 \mathrm{~min}$ (A), $2 \mathrm{~min}$ (B) and $4 \mathrm{~min}$ (C) reoxygenation after HSD onset were analyzed separately. Neuronal synapses lost their function completely within about $120 \mathrm{~s}$ (2 min) after oxygen deprivation $(t=0)$, as seen in the recording traces. Note that due to noise peak effect, zeroamplitudes are not quiet reached in the line graphs. The absence of PSD-95 promoted a somewhat delayed drop of fEPSP amplitude intensities (indicated by arrowheads). (A) WT, $n / m=16 / 9$; PSD$95 \mathrm{KO}, \mathrm{n} / \mathrm{m}=19 / 11$; PSD-93 KO, $\mathrm{n} / \mathrm{m}=15 / 9$; PSD-93/95 DKO, $\mathrm{n} / \mathrm{m}=16 / 11$. (B) $\mathrm{WT}, \mathrm{n} / \mathrm{m}=15 / 9$; PSD-95 KO, $\mathrm{n} / \mathrm{m}=17 / 10$; PSD-93 KO, $\mathrm{n} / \mathrm{m}=18 / 11 ; 93-95 \mathrm{DKO}, \mathrm{n} / \mathrm{m}=15 / 9$. (C) WT, $\mathrm{n} / \mathrm{m}=13 / 9$; PSD-95 KO, $n / m=13 / 10 ;$ PSD-93 KO, $n / m=15 / 8 ; 93-95 \mathrm{DKO}, \mathrm{n} / \mathrm{m}=15 / 9$. 


\subsubsection{PSD-95 KO and PSD-93/95 DKO mice display improved posthypoxic recovery}

More obvious $\mathrm{KO}$ effects were seen during synaptic recovery after reoxygenation as reflected by a return of fEPSP amplitudes. When oxygen was resubmitted 1 minute after the onset of HSD, PSD-95 KO as well as PSD-93/95 DKO slices recovered completely within 9 to 10 minutes, whereas fEPSP amplitudes of WT and PSD-93 KO samples stagnated at about $70 \%$ (WT) to $90 \%$ (PSD-93 KO) below baseline, reaching a plateau thereafter (Figure 12A). Using two-way ANOVA to test for a genotype effect, I detected significant differences comparing PSD-95 KO and PSD-93/95 DKO to control samples, respectively $\left[F_{3,63}=4.204, p=0.009\right.$; PSD-95 KO vs. WT, $p=0.004$; PSD-93/95 DKO vs. WT, $p=0.003$; all other $p$ values $>0.05]$.

In case of reoxygenation after 2 minutes, synaptic recovery of PSD-95 KO and DKO mice was again higher compared to WT, and in addition to PSD-93 KO slices [Figure 12B; $\mathrm{F}_{3,61}=8.458$, $p<0.001$; PSD-95 KO vs. WT, $p=0.003$; PSD-95 KO vs. PSD-93 KO, $p=0.008$; PSD-93/95 DKO vs. WT, $p<0.001$; PSD-93/95 DKO vs. PSD-93 KO, $p<0.001$; all other $p$ values $>0.05]$. Full recovery was achieved slightly later, i.e. at minute 10 to 11 , compared to earlier reoxygenation. In contrast, again WT and PSD-93 KO mice did not recover completely, this time even less by reaching 60 to $70 \%$ of baseline levels.

When oxygen was deprived for 4 minutes upon HSD detection, the acute brain slices were not capable anymore to recover from metabolic stress - at least within the 20 minutes of recovery where recordings have been performed. In fact, synaptic responses of all genotypes remained under $50 \%$ and where hardly distinguishably from noise peaks in the raw traces (Figure 12C). Nonetheless, I obtained significantly stronger recovery for PSD-95 KO and DKO neuronal network $\left[\mathrm{F}_{3,52}=18.391, \mathrm{p}<0.001\right.$; PSD-95 KO vs. WT, $\mathrm{p}<0.001$; PSD-95 KO vs. PSD-93 KO, $p<0.001$; PSD-93/95 DKO vs. WT, $p<0.001$; PSD-93/95 DKO vs. PSD-93 KO, $p<0.001$; all other $p$ values $>0.05$ ] which likely resulted from the higher noise impact on smaller EPSP amplitudes in these mice as specified before.

The here detected impaired synaptic recovery after the longest hypoxic period was implied before by tissue light reflectance, which remained on very high levels even upon reoxygenation after 4 minutes (Figure 8). Accordingly, the recovery of both, synaptic function and physical properties of the brain tissue, was impaired after the extensive hypoxic period.

Highlighted throughout Figure $12 \mathrm{~A}-\mathrm{C}$ is a marked and abrupt peak in synaptic responses shortly after oxygen resupply in DKO and to a smaller extent also in PSD-95 KO mice (indicated by arrowheads). In addition, PSD-93/95 DKO slices showed posthypoxic potentiation with fEPSP amplitudes transiently exceeding the baseline by $10 \%$ for reoxygenation after 1 minute, and 20\% when reoxygenated after 2 minutes, followed by return to prehypoxic levels. These two observations were virtually absent in WT and PSD-93 KO 
mice. Previous studies reported a similar phenomenon, which is referred to as "anoxic LTP" (Crépel et al., 1993; Gozlan et al., 1994). Anoxic LTP delineates hypoxia-induced selective potentiations of the NMDAR-mediated component of synaptic transmission. This persistent form of potentiation is assumed to partially account for delayed neuronal cell death (Crépel et al., 1993; Gozlan et al., 1994). In contrast, the potentiation in PSD-93/95 DKO mice seen here was transient and characterized by increased fEPSP amplitudes, representing both AMPARand, to a lesser extent, NMDAR-mediated transmission. A similar transient potentiation has been reported by Frenguelli (1997) and linked to strong activity of AMPARs and NMDARs during the hypoxic episode. Strong receptor activation is most likely caused by the excess of extracellular glutamate, since its uptake is impaired or even reversed by hypoxia (Szatkowski \& Attwell, 1994). Indeed, the resulting high intracellular $\mathrm{Ca}^{2+}$ levels have been shown to provoke transient potentiations of synaptic transmission (Kauer et al., 1988). Furthermore, HSD-accompanied cell swelling might contribute to the observed potentiation as reported for hyptonia-induced reduction in the interstitial space (Ballyk et al., 1991; Chebabo et al., 1995). Thus, several mechanisms possibly account for transient potentiation in mice lacking both PSD-95 and PSD-93. They all imply a rather unstable neuronal network, in which synaptic activity and cell volume may easily under- and overshoot due to metabolic changes.

Collectively, these results show improved synaptic recovery in PSD-95 KO mice for reoxygenation within less than 4 minutes, demonstrated by faster and more complete reinstatement of normoxic fEPSP amplitudes. This result further strengthens the observed enhanced hypoxia tolerance due to loss of PSD-95, as clearly seen by a delayed onset of HSD (Figure 7) and attenuated change of the HSD-accompanying tissue light reflectance (Figure 8). Additional evidence was given by a trend for decelerated HSD wave propagation (Figure 9) and slightly delayed hypoxia-induced loss of synaptic function in PSD-95 KO mice (Figure 11). Interestingly, all these effects were consistently abolished by additional knockout of the paralog PSD-93, but with the only exception of synaptic recovery. Indeed, mice with a loss of both proteins, PSD-93 and PSD-95, as well showed improved ability for posthypoxic recovery as seen in Figure 11. However, synaptic function in DKO mice seemed to be more unstable and susceptible for changes in oxygen levels as indicated by the abrupt and prominent increase in fEPSP amplitudes shortly after reoxygenation and by transient posthypoxic potentiation during recovery (Figure 12). 
A

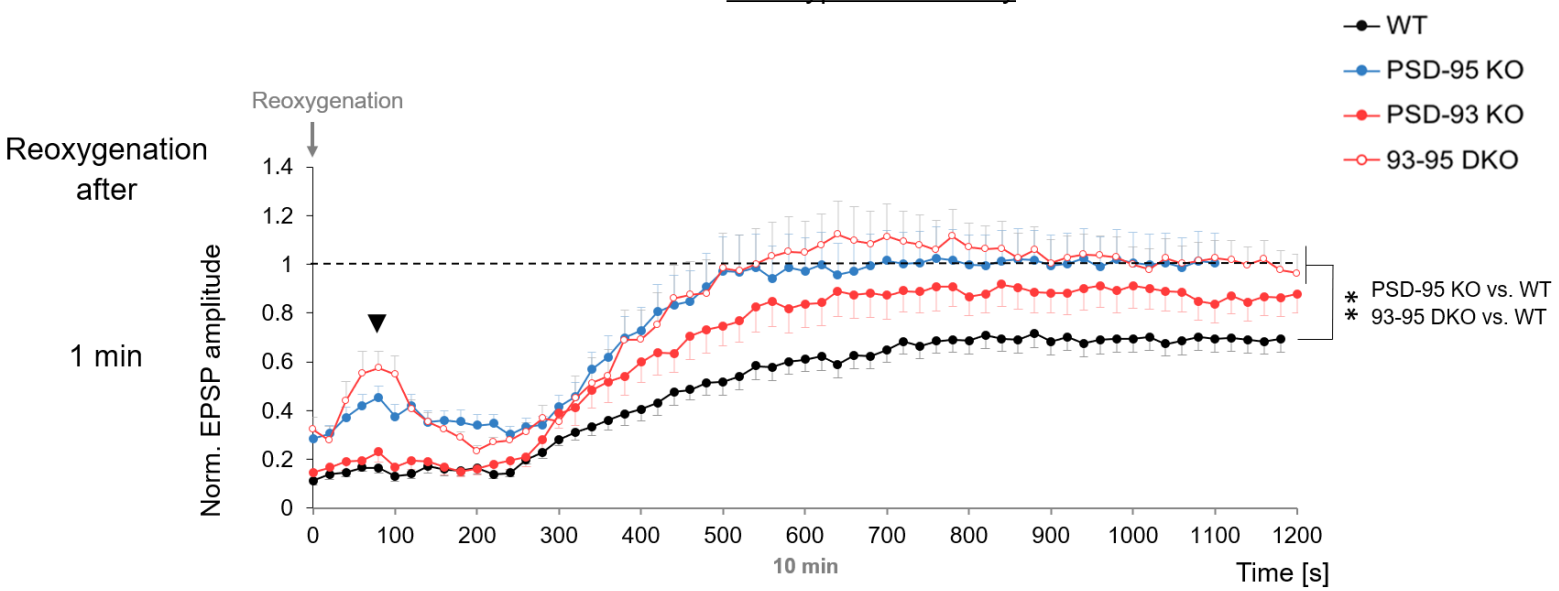

B

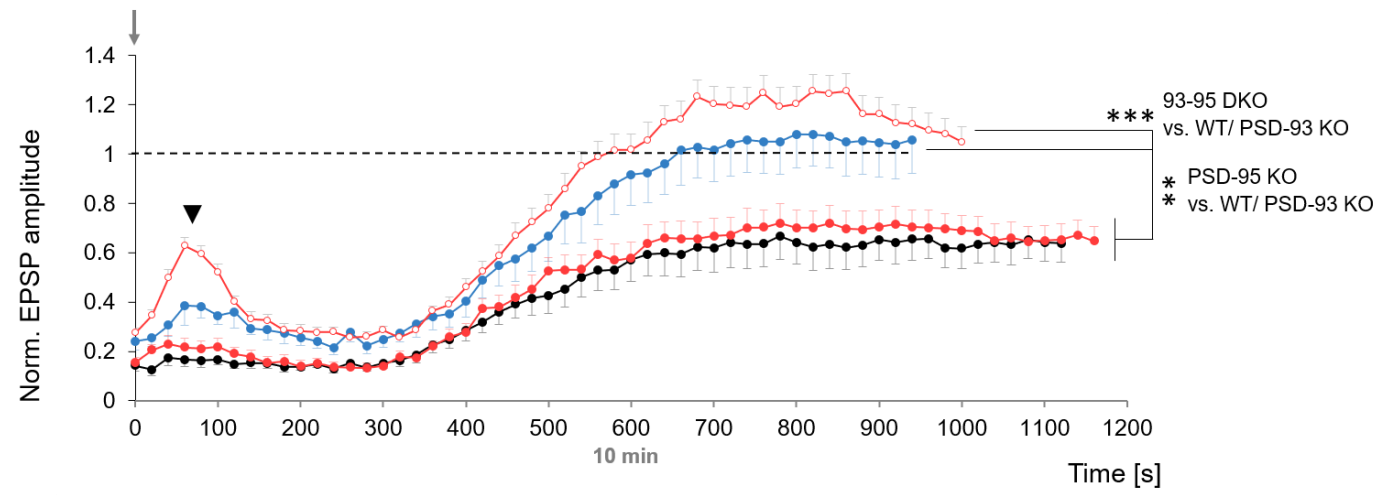

C

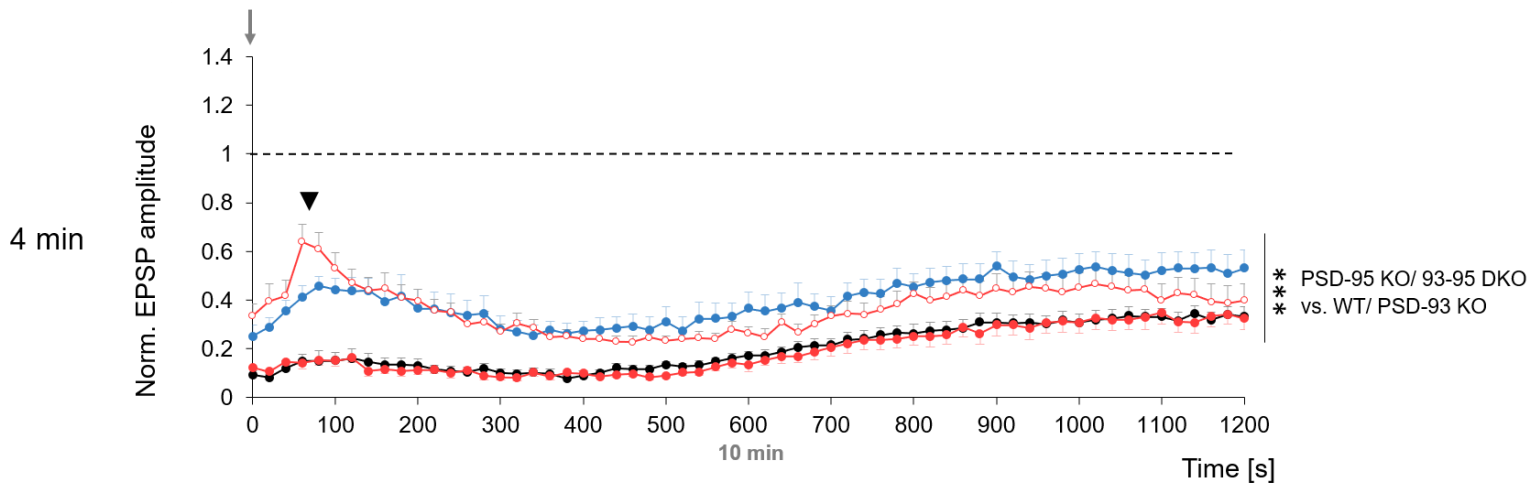

Figure 12: Brain slices from PSD-95 KO and PSD-93/95 DKO mice demonstrated earlier and increased posthypoxic recovery. (A-C) Displayed are time courses for fEPSP amplitudes normalized to prehypoxic baseline after reoxygenation at $t=0$. (A, B) PSD-95 KO and DKO slices showed complete synaptic recovery, whereas WT and PSD-93 KO slices recovered markedly slower and did not return to normoxic baseline levels. (C) When oxygen was resubmitted 4 min after HSD onset, acute brain slices failed to recover within the analyzed time period of 20 min. (A-C) Arrowheads indicate an abrupt amplitude increase detected in DKO and partially in PSD-95 $\mathrm{KO}$ mice. Please note the before mentioned influence of noise peaks on the calculation of amplitude values, wherefore zero-amplitudes at $t=0$ are not quiet reached. (A) $W T, n / m=16 / 9$; PSD-95 KO, $\mathrm{n} / \mathrm{m}=19 / 11$; PSD-93 KO, $\mathrm{n} / \mathrm{m}=16 / 9$; PSD-93/95 DKO, $\mathrm{n} / \mathrm{m}=16 / 11$. (B) $\mathrm{WT}, \mathrm{n} / \mathrm{m}=$ 15/9; PSD-95 KO, $\mathrm{n} / \mathrm{m}=17 / 10$; PSD-93 KO, $\mathrm{n} / \mathrm{m}=18 / 11$; PSD-93/95 DKO, $\mathrm{n} / \mathrm{m}=15 / 9$. (C) WT, $\mathrm{n} / \mathrm{m}=13 / 9 ;$ PSD-95 KO, $\mathrm{n} / \mathrm{m}=13 / 10$; PSD-93 KO, $\mathrm{n} / \mathrm{m}=15 / 8$; PSD-93/95 DKO, $\mathrm{n} / \mathrm{m}=15 / 9$. Twoway ANOVA followed by post-hoc LSD multiple comparison tests. ${ }^{* *} p<0.001 ;{ }^{* *} p<0.01$. All data were given as means \pm SEM. 


\subsection{Synaptic composition of excitatory cortical neurons in MAGUK KO mice}

I then aimed to gain deeper insight into the consequences of PSD-93 and/or PSD-95 KO on synapse composition and function, in order to identify molecular candidates mediating the striking effect of PSD-95 loss on hypoxia susceptibility. PSD-93 as well as PSD-95 are known to play prominent roles in the organization of glutamatergic receptors. Indeed, we previously showed that single loss of PSD-95 or PSD-93 has opposing effects on developmental silent synapse maturation in a variety of cortical areas, including hippocampus (Huang et al., 2015a; D. Favaro et al., in press). Specifically, PSD-95 KO mice maintain a very high level (about $50 \%$ ) of synapses lacking AMPAR-mediated transmission - the so-called "silent synapses" into adulthood, whereas silent synapses in WT mice almost mature completely (Huang et al., 2015a). In contrast, their maturation was accelerated in mice lacking PSD-93, which altogether demonstrates a promoting effect of PSD-95 and an inhibiting effect of PSD-93 for the maturation of silent synapses (D. Favaro et al., in press). Interestingly, in mice lacking both paralogs, the fraction of silent synapses was similar to WT mice as reported until late critical period of primary visual cortex (V1) (D. Favaro et al., in press).

Silent synapses have been proposed to serve as substrates for synaptic plasticity during early refinement of cortical networks (Isaac et al., 1995; Liao et al., 1995; Isaac \& Crair, 1997). Hence, loss of PSD-95 and the resulting high proportion of silent synapses into adulthood virtually maintains this plasticity on the level of juvenile animals, which are known to possess increased capabilities of learning and neuronal network reorganization. Indeed, juvenile ocular dominance (jOD) plasticity was preserved in adult PSD-95 KO mice (Huang et al., 2015a). As younger animals are also known to exhibit higher neuronal regeneration capacities than adults, a plausible link between PSD-95 KO and enhanced hypoxia tolerance lies in the preserved plasticity due to higher number of silent synapses, possibly providing lower susceptibility for hypoxia-induced dysfunction and improved recovery potential.

Since the exact molecular composition of silent synapses and its developmental changes still remain to be clarified, I addressed total as well as surface GluR levels in WT and MAGUK KO mouse brains in different developmental stages in order to reveal potential changes in PSD93- or PSD-95-mediated synaptic processes. 


\subsubsection{Similar cortical protein levels of glutamate and GABA receptors in WT and PSD-95 KO mice}

Huang et al. (2015a) showed that the fraction of V1 silent synapses declined from $~ 55 \%$ at P10-12 before eye opening to about $25 \%$ at the age of P25-30 but remained unchanged in the absence of PSD-95 even into adulthood.

At first, I assessed the developmental change of total GluR and $\mathrm{GABA}_{\mathrm{A}}$ receptor subunit levels in whole-cortex brain slices of WT and PSD-95 KO mice starting from eye opening (P13-14) to 3 weeks (P20-21) and 4 weeks of age (P28-32) (Figure 13). The exact numbers of western blot analysis with normalization to P28-32 levels are provided in Table 13. In order to see the effect of PSD-95 KO on receptor expression, I performed a direct comparison of the absolute values obtained from western blot quantification (Figure 14). At P13-14, protein levels in PSD$95 \mathrm{KO}$ did not differ from control samples in any of the assessed proteins (i.e., GluA1, GluA2, GluN2A, GluN2B and GABA ${ }_{\alpha 1}$; Figure 14). Indeed, Huang et al. (2015a) showed in V1 that the expression of PSD-95 only starts by the age of eye opening. Therefore, PSD-95 KO is unlikely to have pronounced effects on synaptic protein levels at $\mathrm{P} 13-14$ since it is barely expressed at this developmental stage.

Table 13: Developmental profile of glutamatergic and GABAergic receptor subunits in WT and PSD-95 KO cortex.

\begin{tabular}{ccccccc}
\hline \multirow{2}{*}{ Genotype } & \multirow{2}{*}{ Age [P] } & \multicolumn{6}{c}{ Total protein level [norm. to P28-32]; mean \pm SEM } \\
& \multicolumn{7}{c}{ GluA1 } & GluA2 & GluN2A & GluN2B & GABAa1 \\
\hline \multirow{4}{*}{ WT } & $13-14$ & $0.80 \pm 0.06$ & $1.01 \pm 0.05$ & $0.68 \pm 0.04$ & $1.38 \pm 0.02$ & $0.50 \pm 0.09$ \\
& $20-21$ & $1.16 \pm 0.17$ & $1.27 \pm 0.14$ & $0.89 \pm 0.06$ & $1.49 \pm 0.14$ & $0.15 \pm 0.03$ \\
& $28-32$ & $1.00 \pm 0.12$ & $1.00 \pm 0.09$ & $1.00 \pm 0.07$ & $1.00 \pm 0.11$ & $1.00 \pm 0.06$ \\
\hline \multirow{2}{*}{ PSD-95 } & $13-14$ & $0.74 \pm 0.08$ & $1.19 \pm 0.11$ & $0.53 \pm 0.04$ & $1.06 \pm 0.06$ & $0.42 \pm 0.04$ \\
KO & $20-21$ & $0.91 \pm 0.23$ & $1.16 \pm 0.27$ & $0.82 \pm 0.08$ & $1.01 \pm 0.11$ & $0.49 \pm 0.07$ \\
& $28-32$ & $1.00 \pm 0.13$ & $1.00 \pm 0.08$ & $1.00 \pm 0.23$ & $1.00 \pm 0.09$ & $1.00 \pm 0.11$
\end{tabular}


A

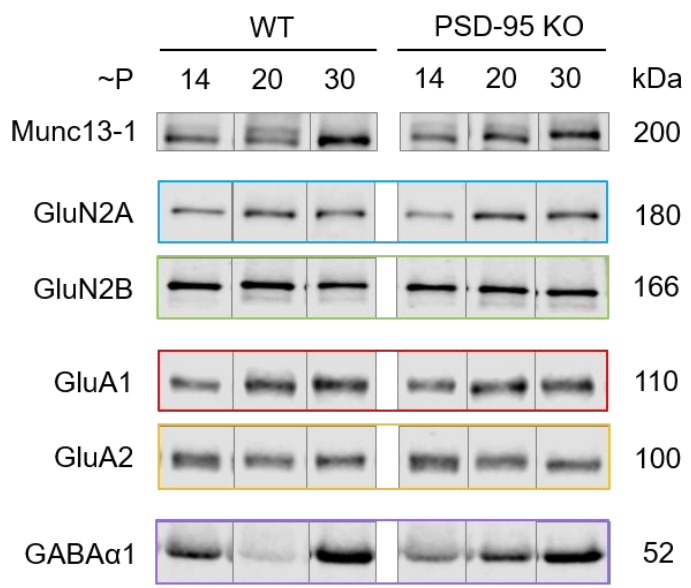

B

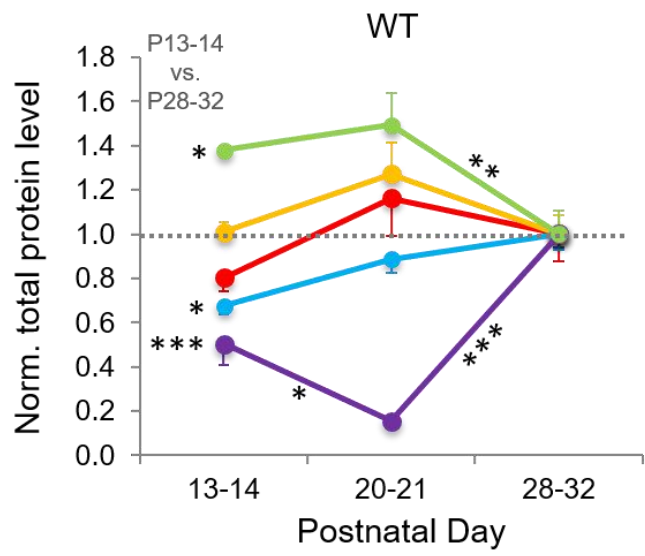

C

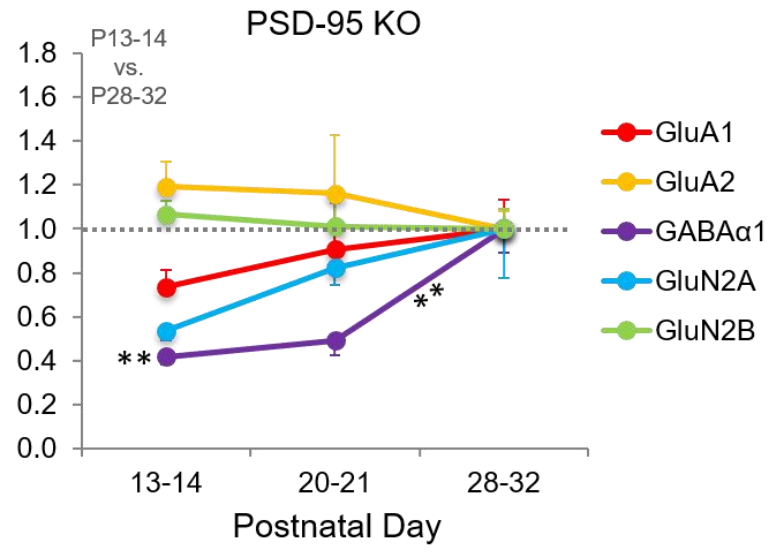

Figure 13: Developmental profile of cortical protein expression in WT and PSD-95 KO brain slices. (A) Western blot analysis showing total levels of receptor subunits in three developmental stages (P13-14, P20-21 and P28-32) of WT and PSD-95 KO mice. Band intensities were normalized to Munc13-1 as a loading control, which also showed a developmental increase but was not different among genotypes. (B, C) Quantified protein levels were normalized to P28-32 as demonstrated in Table 13 and plotted against age for WT (B) and PSD-95 KO (C), respectively. (A-C) WT P13-14, $n=4$; WT P20-21, $n=3$; WT P28-32, $n=6$; PSD-95 KO P13-14, $n=4$; PSD-95 KO P20-21, $n=4$; PSD-95 KO P28-32, $n=5$; for all subunits. One-way ANOVA followed by posthoc LSD multiple comparison tests. ${ }^{* * *} p<0.001$; ${ }^{* *} p<0.01$; ${ }^{*} p<0.05$. All data were given as means \pm SEM. 
A

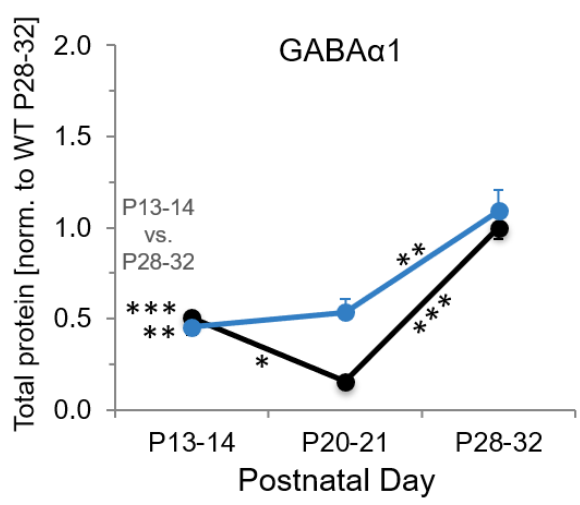

$\rightarrow-W T$

$\rightarrow-P S D-95$ KO

B
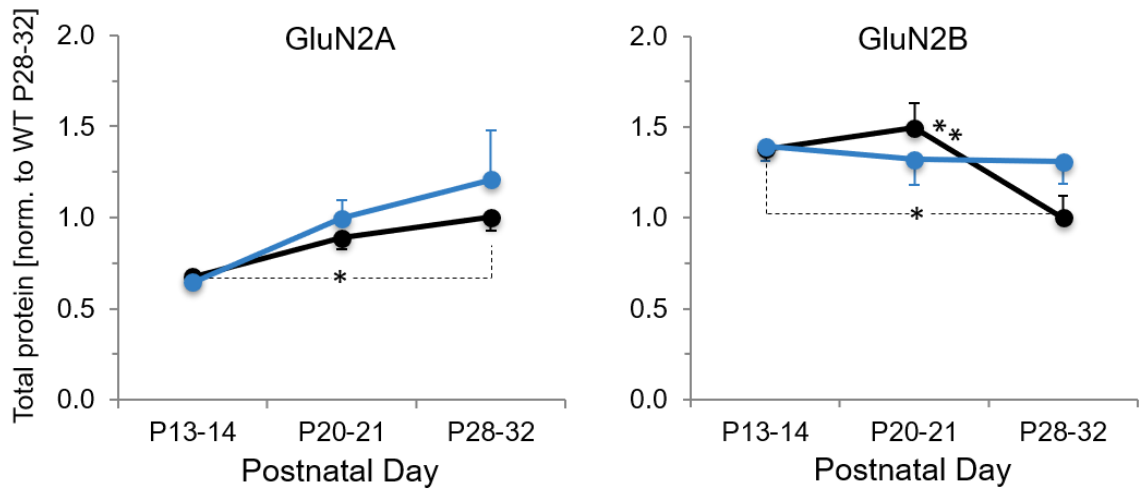

C
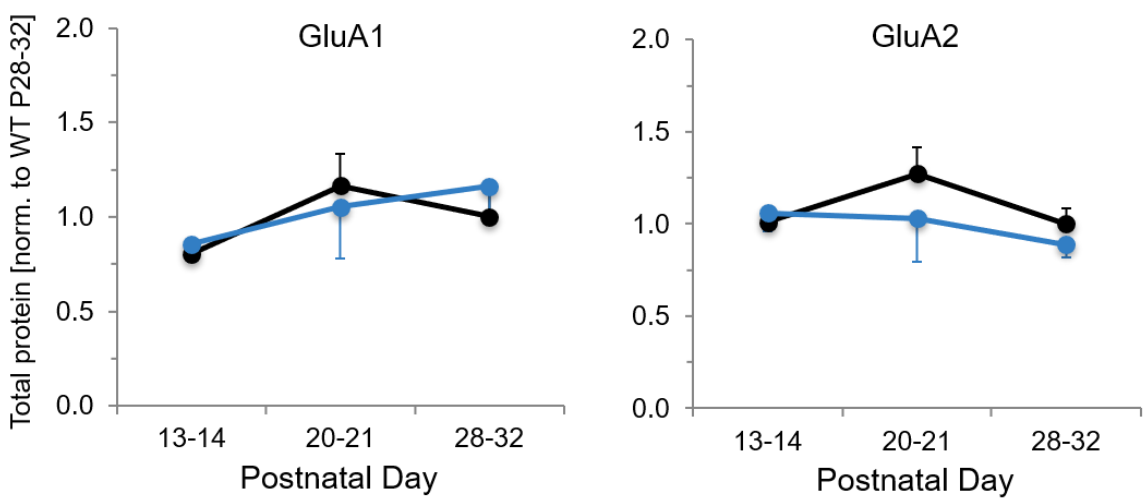

Figure 14: Comparison of glutamate and GABA $_{A}$ receptor levels in WT and PSD-95 KO cortices. (A-C) Quantified protein levels were normalized to WT P28-32 and plotted against age for the GABA a1 subunit (A), NMDAR subunits (B) and AMPAR subunits (C). Significant developmental changes are indicated, and no genotype effect detected. (A-C) WT P13-14, $n=4$; WT P20-21, $n=3$; WT P28-32, $n=6$; PSD-95 KO P13-14, $n=4$; PSD-95 KO P20-21, $n=4$; PSD$95 \mathrm{KO}$ P28-32, $\mathrm{n}=5$; for all subunits. One-way ANOVA followed by post-hoc LSD multiple comparison tests. ${ }^{* *} p<0.001 ;{ }^{* *} p<0.01 ;{ }^{*} p<0.05$. All data were given as means \pm SEM.

As demonstrated in Figure 13, GABA ${ }_{\alpha 1}$ subunit levels in inhibitory synapses showed the steepest rise with $40-50 \%$ at $\mathrm{P} 13-14$ to reference level of $100 \%$ at P28-32, in WT as well as in PSD-95 KO mice [age (ag): WT, $F_{a g(2,10)}=35.650, p_{a g}<0.001 ; P S D-95 \mathrm{KO}, \mathrm{F}_{\mathrm{ag}(2,10)}=$ 15.623, $\left.p_{\mathrm{ag}}=0.001\right]$. However, WT samples displayed a minimum of $15 \%$ protein expression at 3 weeks of age (P20-21), which was absent in PSD-95 KO mice [WT: P13-14 vs. P20-21, p 
$=0.011, \mathrm{P} 13-14$ vs. P28-32, $\mathrm{p}<$ 0.001, P20-21 vs. P28-32, $\mathrm{p}<0.001$; PSD-95 KO: P13-14 vs. $\mathrm{P} 20-21, \mathrm{p}>0.05, \mathrm{P} 13-14$ vs. P28-32, $\mathrm{p}=0.001, \mathrm{P} 20-21$ vs. $\mathrm{P} 28-32, \mathrm{p}=0.001]$. The observed sharp increase of subunit expression starting at P20-21 is in parallel to the reported pivotal role of inhibitory interneurons in triggering the onset of critical period plasticity in the visual cortex (Huang et al., 1999; Fagiolini \& Hensch, 2000). Comparison of both genotypes among all developmental stages showed a trend for generally higher GABA ${ }_{\alpha 1}$ levels in PSD-95 KO mice, while the developmental trajectories were highly comparable [Figure 14A; genotype (gt): $F_{g t(1,20)}=4.271, p_{g t}=0.052$ (trend); $\left.F_{a g(2,20)}=44.529, p_{a g}<0.001 ; p_{g t x a g}>0.05\right]$.

Expression of the NMDAR subunit GluN2A increased progressively with age, possibly due to growth and/or linkage of new excitatory synapses after eye opening. The developmental increase reached significance for WT mice but not for PSD-95 KO mice, presumably due to the relatively small $n$-number and high variance [Figure 13B/C; WT: $F_{a g(2,10)}=6.348$, $p_{a g}=0.017 ; \mathrm{P} 13-14$ vs. P28-32, $p=0.005$, all other $p$ values $>0.05 ;$ PSD-95 KO: $p_{a g}>0.05$ (note high SEM for P28-32)]. By comparing both genotypes, GluN2A expression was unaffected in the absence of PSD-95 [Figure 14B; $p_{g t}>0.05 ; F_{a g(2,20)}=4.972, p_{a g}=0.018$; $\left.p_{\text {gtxag }}>0.05\right]$.

In contrast to the developmental increase of GluN2A levels, GluN2B subunits declined markedly in WT mice from $\sim 140 \%$ at $\mathrm{P} 13-14$ to $\mathrm{P} 28-32$ reference levels with a slight peak at P20-21, whereas in PSD-95 KO mice, the developmental change was absent [Figure 14B; $p_{g t}>0.05 ; F_{a g(2,20)}=3.455, p_{a g}=0.051$ (trend), $p_{g t x a g}>0.05 ; W T: F_{a g(2,10)}=6.651, p_{a g}=0.015 ;$ $\mathrm{P} 13-14$ vs. $\mathrm{P} 20-21, \mathrm{p}>0.05, \mathrm{P} 13-14$ vs. $\mathrm{P} 28-32, \mathrm{p}=0.021, \mathrm{P} 20-21$ vs. $\mathrm{P} 28-32, \mathrm{p}=0.009$; PSD-95 KO: $\left.p_{a g}>0.05\right]$. The GluN2B drop is in accordance with the reported developmental switch of GluN2B- to GluN2A-containing NMDARs in the cerebral cortex - triggered by receptor activity and sensory experience (Monyer et al., 1994; Sheng et al., 1994). As the GluN2B expression remained unchanged in PSD-95 KO samples, the subunit exchange seemed to be impaired, which is presumably linked to the lack of silent synapse maturation after eye opening. This observation is in consistent with a previous study showing that PSD-95 is essential for the replacement of GluN2B- with GluN2A-containing NMDARs (Elias et al., 2008).

GluA1 levels non-significantly increased from $\sim 80 \%$ as shown in Figure $13 \mathrm{~B} / \mathrm{C}$ and did not differ significantly among the genotypes (Figure 14C), though displaying a slight peak at $\sim 115 \%$ in WT cortices $\left[p_{g t}>0.05 ; p_{a g}>0.05 ; p_{g t x a g}>0.05\right.$; one-way ANOVA: $p_{a g}>0.05$ for WT and PSD-95 KO]. Descriptively, GluA2 already reached the expression level of P28-32 at eye opening in control slices, also peaked at P20-21 and declined afterwards (Figure 13B). Levels in PSD-95 KO slices stayed virtually stable, showing a slight decrease starting at $\sim 120 \%$ (Figure 13C). However, these changes were not significant $\left[p_{a g}>0.05\right.$ for WT and PSD-95 $\mathrm{KO}$. As displayed in Figure $14 \mathrm{C}$, also no significant differences among genotypes were detected $\left[p_{g t}>0.05 ; p_{a g}>0.05 ; p_{g t x a g}>0.05\right]$. Therefore, these results suggest similar cortical 
AMPAR expression in WT and PSD-95 KO mice. Taking into account the reported 50\% of AMPAR transmission-lacking synapses in P25-30 PSD-95 KO mice compared to only $25 \%$ in WT samples (V1, Huang et al., 2015a), the question arises as to whether AMPAR subunits are still expressed to normal levels in the absence of PSD-95 but are not transported to the cell surface. To further address this question, I analyzed the surface levels of glutamate receptors.

\subsubsection{Slight effects on GluR surface expression due to the absence of PSD-95}

In order to identify the receptor fraction being expressed on the cell surface, cortical brain slices were incubated with the membrane-impermeable, bifunctional crosslinker BS3 (Figure 15). The BS3 reagent covalently crosslinks extracellular lysine residues of surface-expressed proteins, hence increasing their apparent molecular weight on immunoblots (Boudreau et al., 2012). On the contrary, intracellular receptors remain unmodified and can be detected as monomers. Since the crosslinked protein fraction did not enter the SDS-resolving gel, I directly calculated the protein surface levels from the intracellular pool as follows:

$$
\text { Protein surface level }=1-\frac{\text { internal level }(\text { monomer band in sample }+B S 3)}{\text { total level }(\text { monomer band } w / o B S 3)}
$$

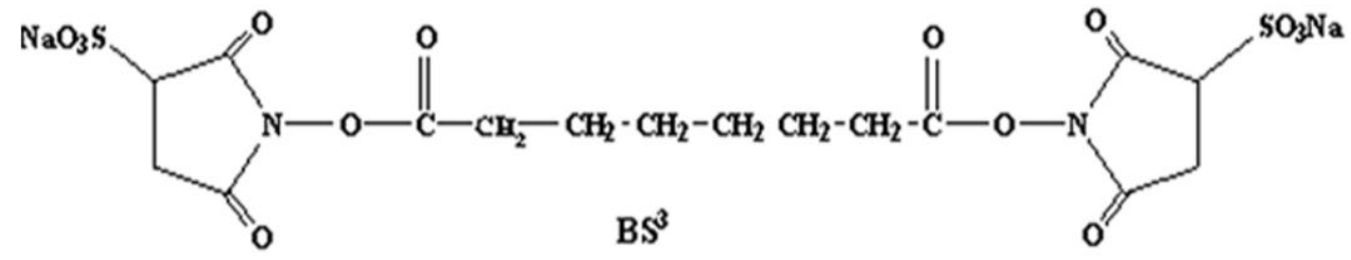

Figure 15: Molecular structure of the crosslinker BS3. BS3 (Bis(sulfosuccinimidyl)suberate) covalently binds to primary amines of extracellular lysine side chains and/or $\mathrm{N}$-terminals via its two reactive NHS ( $\mathrm{N}$-hydroxysuccinimide)-ester groups. Therefore, BS3 crosslinks proteins such as receptors expressed on the cell surface. Image adapted from Shi et al. (2017).

At first, I compared AMPAR as well as NMDAR surface levels in cortical slices of WT, PSD-95 KO, PSD-93 KO and PSD-93/95 DKO mice at 4 weeks of age (P28-32). The corresponding surface protein levels are provided in Table 14. 
Table 14: Cortical surface levels of AMPAR and NMDAR subunits in WT and MAGUK KO mice.

\begin{tabular}{ccccccc}
\hline \multirow{2}{*}{ Genotype } & $\begin{array}{c}\text { Age } \\
\text { [P] }\end{array}$ & \multicolumn{5}{c}{ Surface protein level [\%]; mean \pm SEM } \\
& & GluA1 & GluA2 & GluN2A & GluN2B & GABA $_{\alpha 1}$ \\
& $13-14$ & $63.44 \pm 4.71$ & $53.97 \pm 3.85$ & $43.81 \pm 3.58$ & $35.15 \pm 4.13$ & $63.01 \pm 1.54$ \\
WT & $20-21$ & $70.79 \pm 2.86$ & $79.83 \pm 0.71$ & $28.67 \pm 3.47$ & $41.60 \pm 1.35$ & $54.22 \pm 1.88$ \\
& $28-32$ & $63.09 \pm 2.90$ & $73.60 \pm 1.08$ & $49.29 \pm 2.30$ & $35.22 \pm 4.20$ & $62.48 \pm 2.22$ \\
\hline PSD-95 & $13-14$ & $56.20 \pm 1.59$ & $55.04 \pm 0.91$ & $46.96 \pm 6.34$ & $41.10 \pm 10.1$ & $64.60 \pm 4.26$ \\
KO & $20-21$ & $62.57 \pm 2.84$ & $67.77 \pm 1.41$ & $50.62 \pm 5.42$ & $42.24 \pm 7.92$ & $68.88 \pm 1.07$ \\
& $28-32$ & $53.68 \pm 3.49$ & $70.33 \pm 3.07$ & $37.37 \pm 4.96$ & $41.13 \pm 4.71$ & $61.71 \pm 1.06$ \\
\hline PSD-93 & $28-32$ & $52.39 \pm 5.12$ & $69.79 \pm 2.61$ & $41.55 \pm 2.69$ & $36.21 \pm 2.05$ & - \\
KO & & & & & & \\
\hline 93/95 & $28-32$ & $52.67 \pm 4.14$ & $68.38 \pm 4.04$ & $39.92 \pm 3.86$ & $33.32 \pm 6.03$ & - \\
DKO & & & & & &
\end{tabular}

The AMPAR subunits GluA1 and GluA2 were expressed on the membrane with $60-70 \%$ of total in WT mice at 4 weeks of age, whereas only $~ 50 \%$ of GluN2A and $~ 35 \%$ of GluN2B were membrane-located. As demonstrated in Figure 16B/C, MAGUK KO mice did not exhibit significantly altered surface levels neither of the NMDAR, nor of AMPAR subunits as compared to WT mice [P28-32: GluA1, $F_{g t(3,22)}=1.341, p_{g t}=0.287 ; G l u A 2, F_{g t(3,22)}=0.348, p_{g t}$ $=0.791 ;$ GluN2A, $\left.F_{g t(3,22)}=1.770, p_{g t}=0.182 ; G l u N 2 B, F_{g t(3,19)}=0.636, p_{g t}=0.601\right]$. On the descriptive level, the surface expression of GluA1, GluA2 and GluN2B was slightly reduced in all KO mice compared to WT.

Notably, the beforehand reported total AMPA receptor levels (section 3.2.1) were expressed to similar amounts on the cell surface among the genotypes, therefore providing no explanatory hints for the $\sim 25 \%$ more AMPAR-transmission deficient synapses in PSD-95 KO mice at this developmental stage. 
A

P28-32

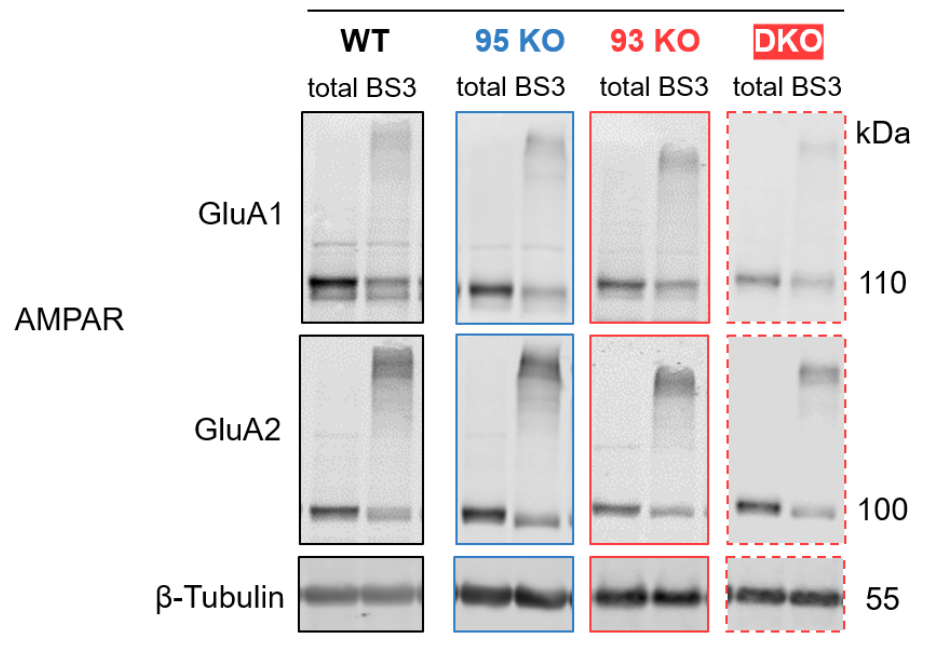

B
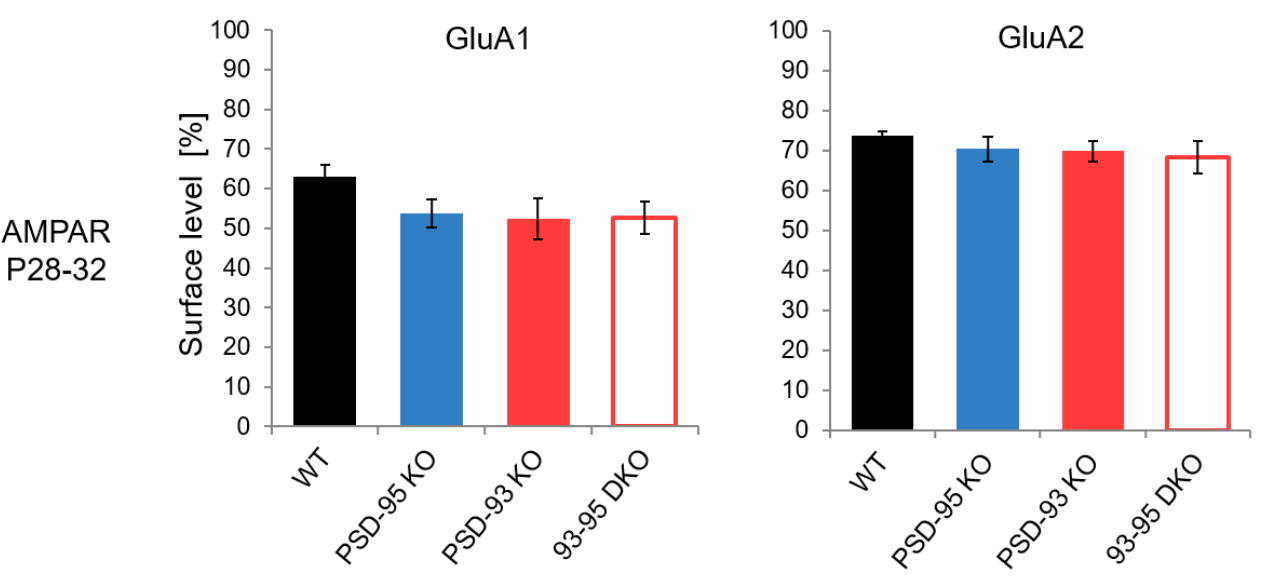

C
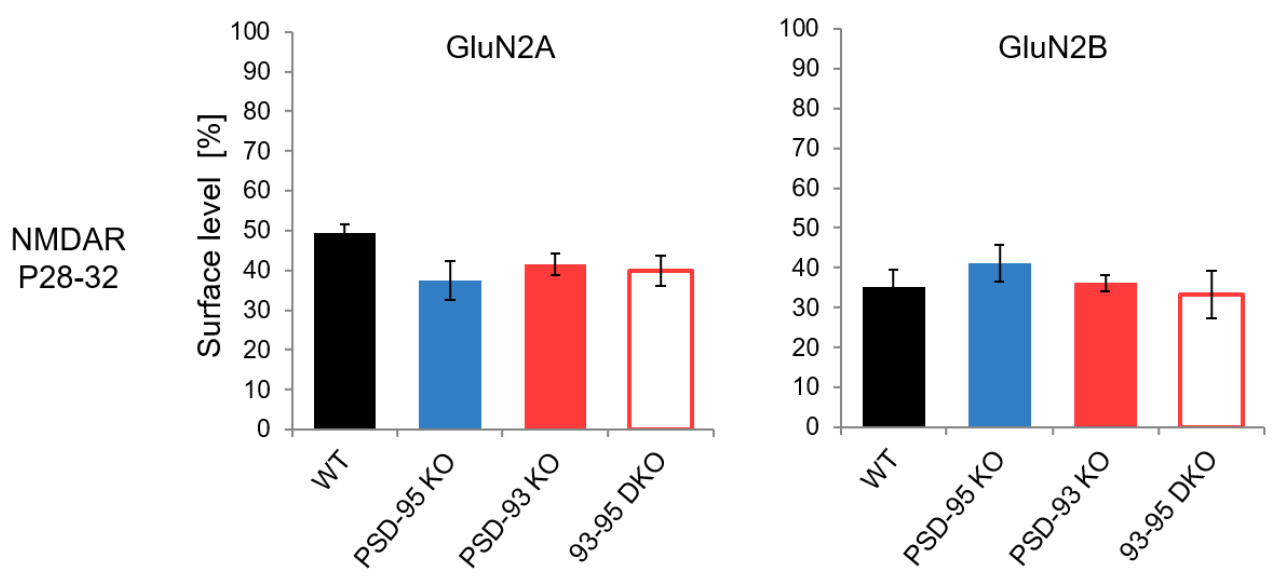

Figure 16: Surface levels of AMPAR and NMDAR subunits in WT and MAGUK KO mice at P28-32. (A) Representative western blots of total and BS3-crosslinked GluA1 and GluA2. Crosslinked proteins are visible in the "BS3" lane as smears of approximately $>400 \mathrm{kDa}$, a result in line with crosslinked subunits (each $\sim 100 \mathrm{kDa}$ ) of a GluR tetramer and possible inclusions of small auxiliary proteins (Boudreau et al., 2012). On the other hand, intracellular receptors were detected as monomers at the indicated characteristic height $(100 / 110 \mathrm{kDa})$ and their bands quantified for surface level calculation. $\beta$-Tubulin was used as a loading control. (B) Resulting surface levels of GluA1 and GluA2 in cortex of WT and MAGUK KO mice at P28-32. (C) Bar graph displaying the fractions of GluN2A and GluN2B expressed on the cell surface at P28-32. (B-C) WT, $n=5-6$; 
PSD-95 KO, $n=6-8$; PSD-93 KO, $n=7$; PSD-93/95 DKO, $n=4$. One-way ANOVA, $p>0.05$. All data were given as means \pm SEM.

To assess putative differences in the developmental profile of the surface expression between WT and PSD-95 KO, I also determined AMPAR, NMDAR and GABA $A_{A}$ receptor surface levels at P13-14 ( eye opening) and 3 weeks of age (P20-21). The respective values are provided in Table 14.

The GluA1 proportion on the cell surface of PSD-95 KO neurons demonstrated exactly the same developmental trace as in WT mice, though with significantly reduced surface levels by $7-10 \%$ among all age groups [Figure 17A; $F_{g t(1,24)}=8.021, p_{g t}=0.009 ; p_{a g}>0.05 ; p_{g t x a g}>0.05$; one-way ANOVA: $p_{a g}>0.05$ for WT and PSD-95 KO]. Despite the reduced GluA1 surface levels by loss of PSD-95, a reduction of solely $7-10 \%$ is much lower than the $25 \%$ discrepancy in silent synapse levels reported, at least for P28-32. In case of GluA2, a developmental increase in surface localization was detected for both genotypes [WT: $F_{a g(2,10)}=$ 32.085, $\mathrm{pag}<0.001$; P13-14 vs. P20-21, $\mathrm{p}<0.001, \mathrm{P} 13-14$ vs. P28-32, $\mathrm{p}<0.001, \mathrm{P} 20-21$ vs. P28-32, $p>0.05$; PSD-95 KO: $F_{a g(2,14)}=9.267, p_{a g}=0.003 ; P 13-14$ vs. $P 20-21, p=0.010$, $\mathrm{P} 13-14$ vs. P28-32, $\mathrm{p}=0.001, \mathrm{P} 20-21$ vs. P28-32, $\mathrm{p}>0.05]$. Again, GluA2 surface levels were only slightly - but significantly - decreased in PSD-95 KO mice, mainly at P20-21 with 12\% difference $\left[F_{g t(1,24)}=4.697, p_{g t}=0.040 ; F_{a g(2,24)}=31.256, p_{a g}<0.001 ; p_{g t x a g}>0.05\right]$. By considering the similar amount of silent synapses in WT and PSD-95 KO mice before eye opening, only reaching the $25 \%$ difference by the age of P25 (in V1, Huang et al., 2015a), one would have assumed that AMPAR surface levels remained constant in the KO mice while increasing in WT by surface-incorporation during silent synapse maturation.

No such diminished surface levels among all age groups were found in PSD-95 KO mice compared to WT by assessing NMDA or GABA subunits (Figure $17 \mathrm{~B} / \mathrm{C}$ ). In contrast, surface expression of GluN2A as well as GABA ${ }_{\alpha 1}$ was slightly enhanced in PSD-95 KO slices at 3 weeks of age, while showing a significant minimum in the control samples [(i) GluN2A: $p_{g t}>0.05 ; p_{a g}>0.05 ; F_{g t x a g(2,23)}=5.898, p_{g t x a g}=0.009 ;$ one-way ANOVA, WT: $F_{a g(2,10)}=$ 11.116, $\mathrm{p}_{\mathrm{ag}}=0.003 ; \mathrm{P} 13-14$ vs. $\mathrm{P} 20-21, \mathrm{p}=0.010, \mathrm{P} 13-14$ vs. $\mathrm{P} 28-32, \mathrm{p}>0.05, \mathrm{P} 20-21$ vs. P28-32, $p=0.001$; PSD-95 KO: $p_{a g}>0.05$. (ii) $\underline{G A B A}_{\alpha 1}: F_{g t(1,24)}=6.816, p_{g t}=0.015 ; p_{a g}>0.05$; $F_{\text {gtxag(2,24) }}=5.359, p_{\text {gtxag }}=0.012$; one-way ANOVA, WT: $F_{a g(2,10)}=4.207, p_{a g}=0.047 ; P 13-14$ vs. P20-21, $\mathrm{p}=0.027, \mathrm{P} 13-14$ vs. P28-32, $\mathrm{p}>0.05$, P20-21 vs. P28-32, $\mathrm{p}=0.025$; PSD-95 KO: $\left.p_{a g}>0.05\right]$. The surface levels of GluN2B were mostly stable and did not differ among the genotypes $\left[p_{g t}>0.05 ; p_{a g}>0.05 ; p_{g t x a g}>0.05\right.$; one-way ANOVA: $p_{a g}>0.05$ for WT and PSD$95 \mathrm{KO}$.

Together, these data suggest that silent synapses likely contain similar levels of AMPA receptors with only partly and minimal surface reductions depending on the developmental stage, but clearly not reaching $25 \%$ reduction at 4 weeks of age. Therefore, another possibility 
is that surface AMPARs are either located extrasynaptically or inactivated (or both), resulting in the lack of AMPAR-transmission in silent synapses.

A
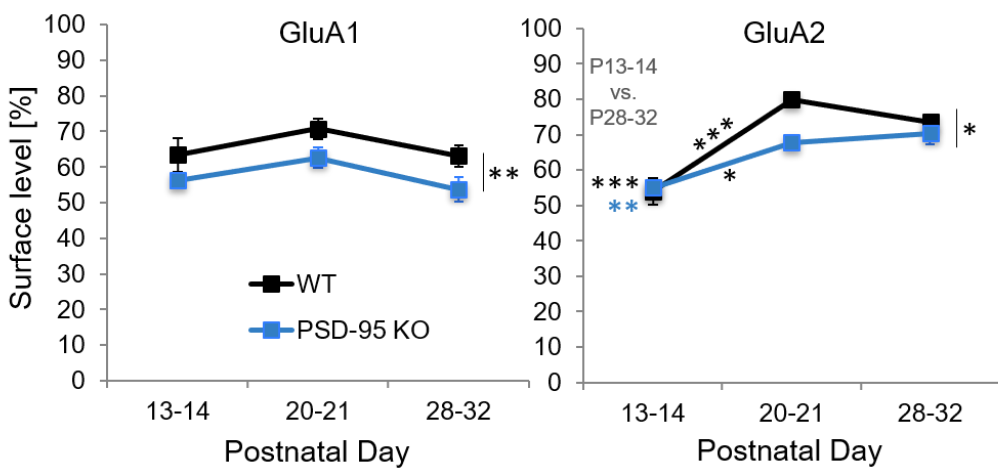

D

B
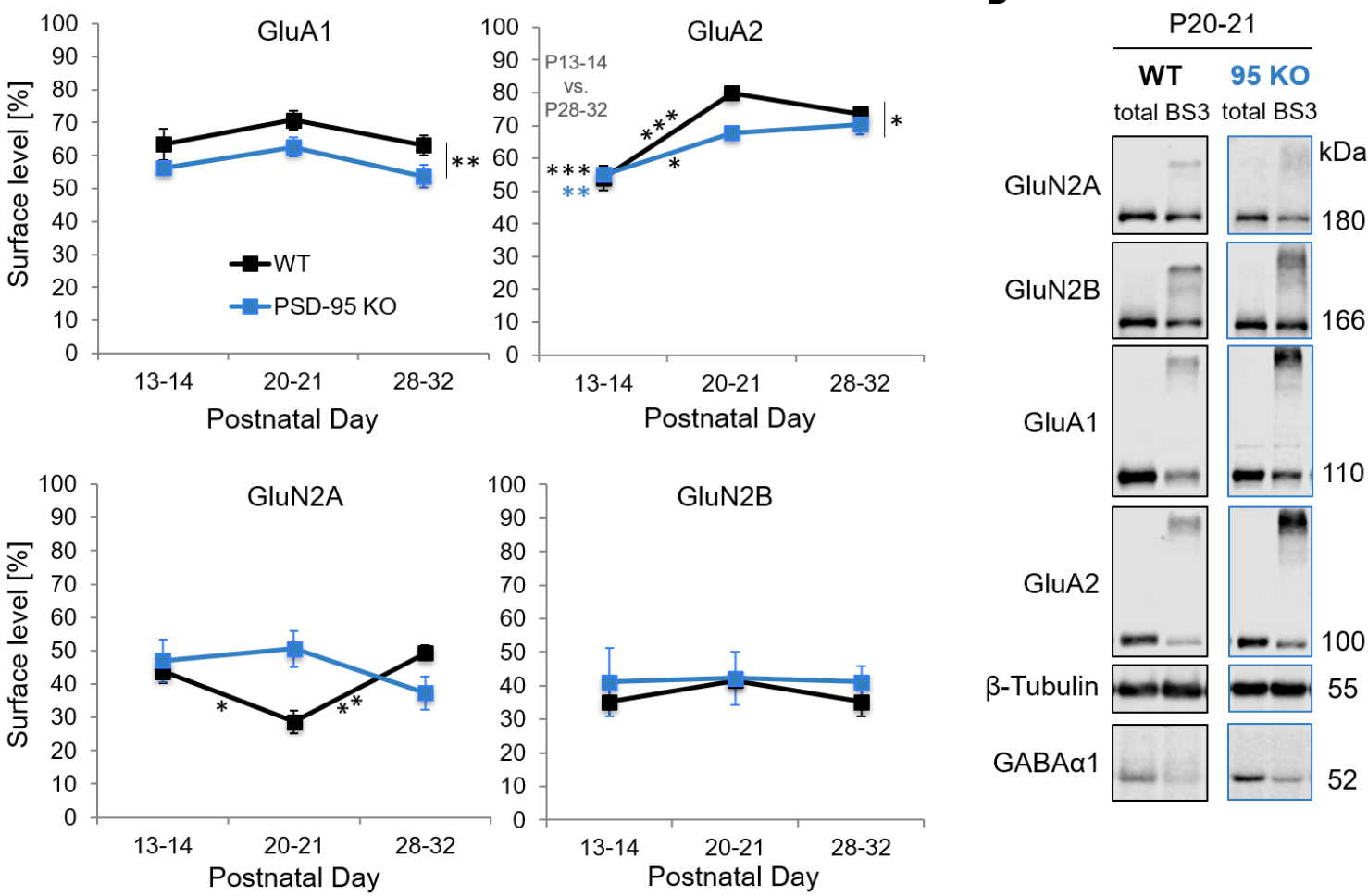

C
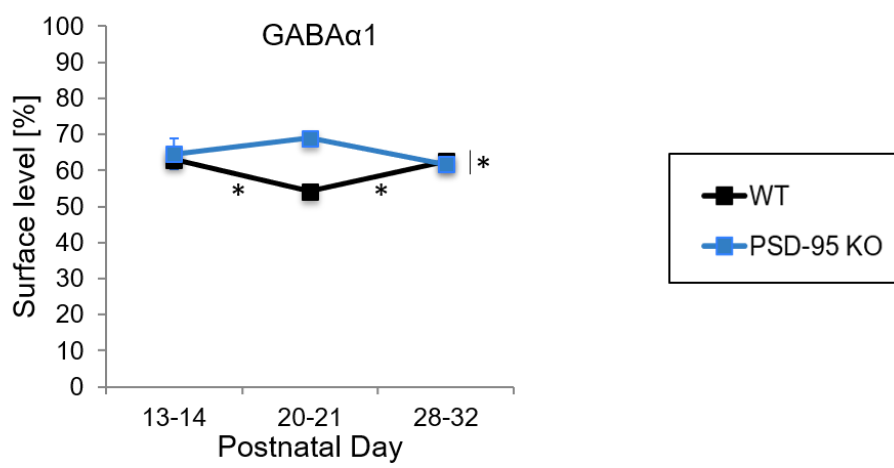

Figure 17: Developmental profile of AMPAR, NMDAR and GABA $A_{A}$ receptor surface expression in WT and PSD-95 KO cortices. Surface levels of AMPAR subunits (A), NMDAR subunits (B) and the GABA-A a1 subunit (C) among three developmental stages as indicated at the $x$-axis. Ratios were obtained by quantification of immunoblot monomer bands as exemplarily shown in (D). Crosslinked proteins were visible as smears of higher molecular weight in the "BS3" lanes. (A-D) WT P13-14, $n=4$; WT P20-21, $n=3$; WT P28-32, $n=5-6$; PSD-95 KO P13-14, $n=4-$ 5; PSD-95 KO P20-21, $\mathrm{n}=4$; PSD-95 KO P28-32, $\mathrm{n}=6$-8. Two-way ANOVA (genotype)/ one-way ANOVA (age) followed by post-hoc LSD multiple comparison tests, ${ }^{* * *} p<0.001 ;{ }^{* *} p<0.01$; ${ }^{*} p<$ 0.05 . All data were given as means \pm SEM. 


\subsubsection{Investigation of the TCM effect on AMPAR-mEPSC frequency}

As mentioned above, the molecular characteristics of silent synapses still remain elusive. Since the total and surface expression of AMPA receptors seemed to be virtually unaltered in PSD-95 KO mice (section 3.2.1 and 3.2.2), which yet show high levels of AMPAR-silent synapses (Huang et al., 2015a), such AMPARs might be rather inactive or located extrasynaptically. The latter assumption is supported by lower levels of the AMPAR subunits GluA1, GluA2 and GluA3 in PSD fractions of adult PSD-95 KO mice detected in V1 (Huang et al., 2015a). In detail, synaptic levels were reduced to about $50 \%$, which is equal to the $\sim 50 \%$ silent synapse number in adult PSD-95 KO mice, therefore implicating that these synapses do not contain PSD-located AMPARs at all.

Considering the developmental decrease of silent synapse number in WT mice and by assuming that silent synapses mature to AMPAR-transmitting synapses, one would expect a correlating increase in AMPAR miniature-EPSC (mEPSC) frequency. mEPSCs result from spontaneous vesicle release and are directly proportional to the number of AMPAR-positive synapses, because presumably one synapse just releases one vesicle at a time without electric stimulation (Frerking et al., 1997). As expected, mEPSC frequency in V1 of PSD-95 $\mathrm{KO}$ mice is reduced as compared to WT mice and remains stable after eye opening - due to the lack of silent synapse maturation (Supporting Figure 21A, appendix). Surprisingly, we and others (Han et al., 2017) found that mEPSC frequency as well remains stable in WT mice, although silent synapses mature to AMPAR-transmitting synapses. This finding indicates another mechanism for the homeostasis of AMPAR transmission in the WT brain. We therefore previously investigated a possible role of AMPAR desensitization and found that the desensitization blocker TCM (trichlormethiazide) promoted a more than three-fold increase in mEPSC frequency in WT mice at the age of P31, while this effect was lower in an earlier developmental stage (P16) (Supporting Figure 21B, Appendix). Hypothetically, AMPAR homeostasis could be achieved by a specific mechanism accompanying silent synapse maturation, which results in a proportion of spontaneously non-active AMPARs. These nonactive or "permanently desensitized" AMPARs might be re-activated due to TCM treatment. This effect could in general be pre- or postsynaptic. To address its localization, I used TCM in a Synaptotagmin 1 (Syt1) antibody uptake assay in dissociated hippocampal mouse culture for monitoring presynaptic vesicle release as illustrated and mechanistically described in Figure 18. 


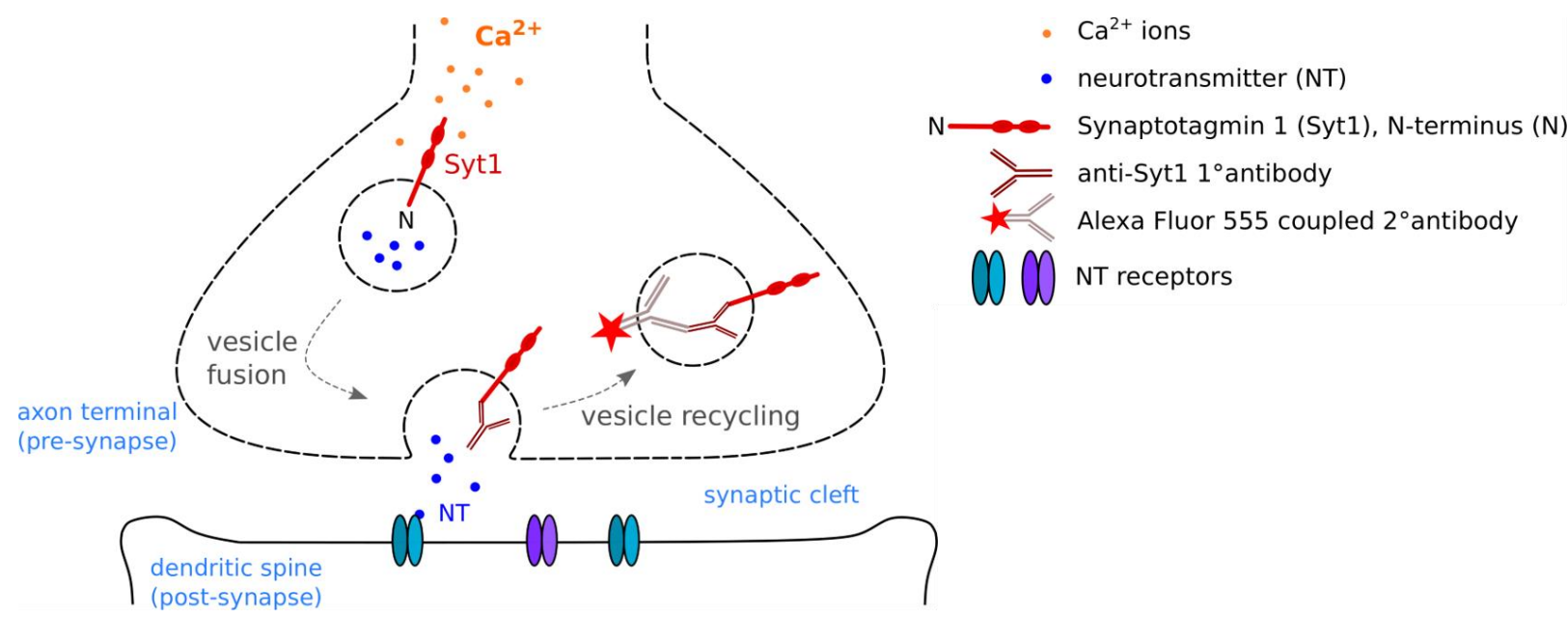

Figure 18: Molecular mechanism of the Synaptotagmin 1 uptake assay for monitoring presynaptic activity. The release of presynaptic vesicles can be monitored by an uptake or "feeding" assay of Syt1, a membrane-trafficking protein acting as a calcium sensor (Südhof, 2002). As illustrated here, intracellular calcium ions trigger vesicle fusion with the presynaptic membrane in order to release neurotransmitters into the synaptic cleft. As soon as the N-terminus of Syt1 is exposed to the extracellular space, the primary antibody can bind and gets incorporated into the vesicle following recycling. Therefore, permeabilization of the cell membranes (using Triton X-100) was necessary in order to couple the primary antibody with a fluorescence-labeled secondary antibody. In case of a high fusion rate of presynaptic vesicles, strong fluorescence signals were detected and vice versa.

\subsubsection{Synaptotagmin 1 co-localizes with the presynaptic markers}

\section{Munc13, VGLUT1 and Ankyrin-B}

Apart from its localization in presynaptic vesicle membranes, Syt1 was also reported to be present at the postsynaptic, dendritic site and linked to AMPAR exocytosis during LTP (Wu et al., 2017). Hence, I first sought to confirm that the assay demonstrates presynaptic (but not postsynaptic) activity by immunofluorescence co-staining of Syt1 with specific synaptic markers in primary mouse hippocampal culture at 15 days in vitro (DIV).

As demonstrated in Figure 19A, Syt1 co-localized with the active zone marker Munc13-1, while both proteins were separated but in close contact to postsynaptic dendrites visualized by staining of the microtubule-associated protein (MAP) 2. Furthermore, Syt1 signals were colocalized with the vesicular glutamate transporter (VGLUT) 1 in synaptic vesicle membranes of excitatory neurons (Figure 19B). Together with the co-localization of Syt1 and Ankyrin B, which is reported to be highly enriched in axons (Kunimoto, 1995; Engelhardt et al., 2013) (Figure 19C), these results show that the Syt1 uptake assay can be used to determine a possible presynaptic origin of the TCM effect. 
A1
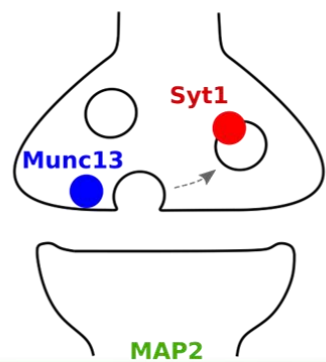

B1
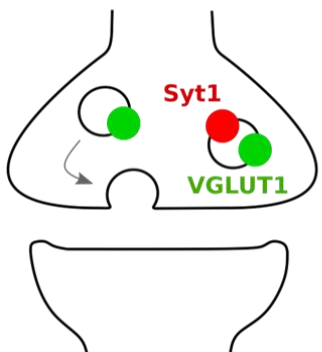

C1
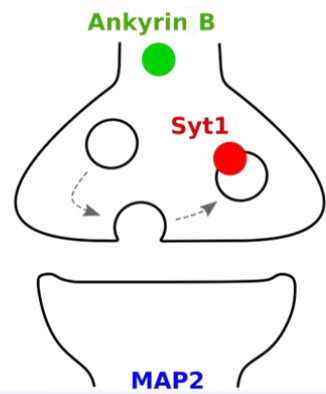

A2

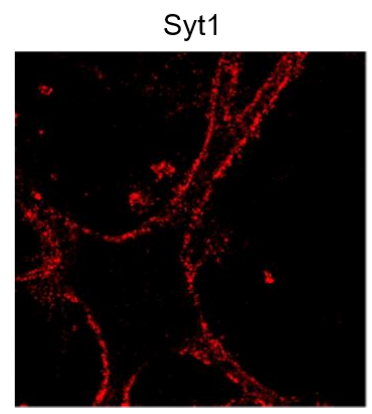

Munc13-1 (active zone)

MAP2 (dendrites)
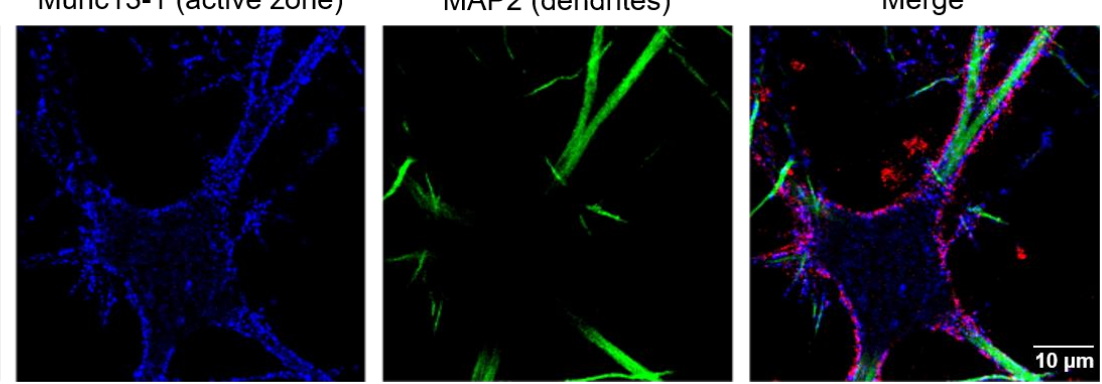

B2
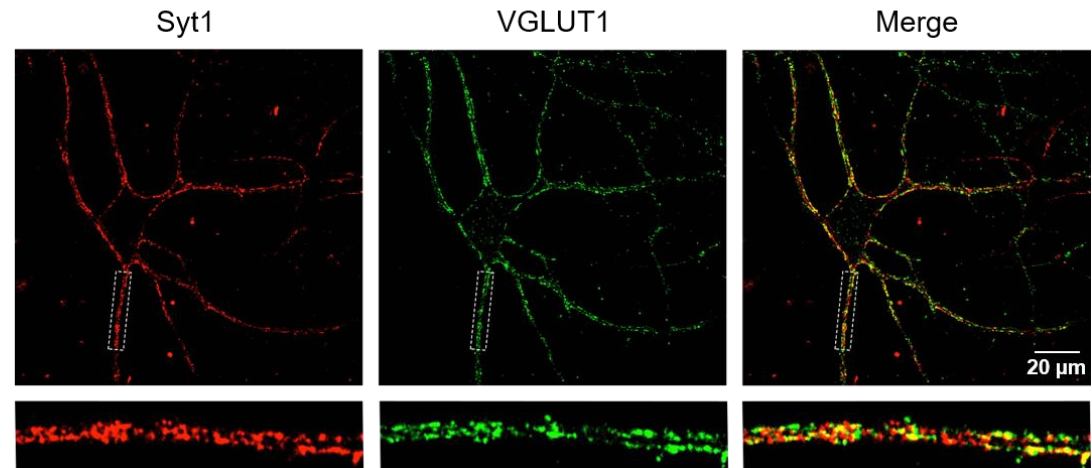

C2

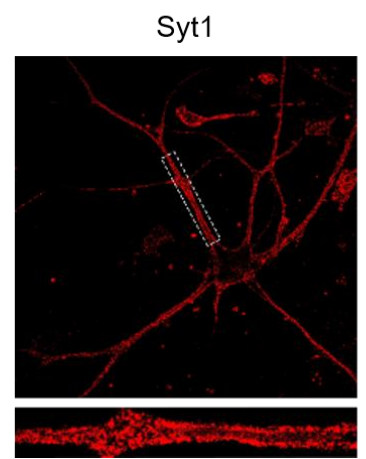

Ankyrin B (axons)

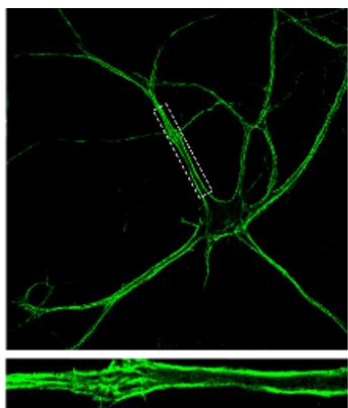

MAP2 (dendrites)

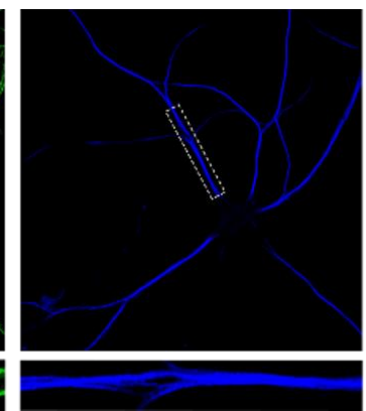

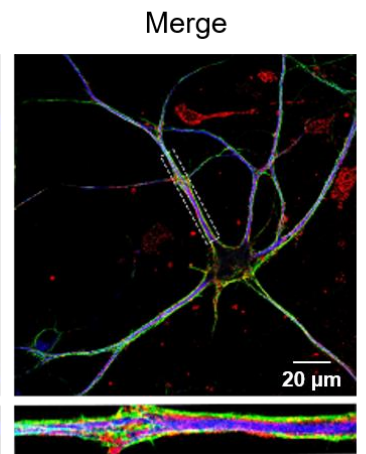

Figure 19: Immunofluorescence staining showed co-localization of Synaptotagmin 1 with diverse presynaptic markers. Dissociated primary hippocampal cultures prepared from WT P0mice were immunolabeled at 15 DIV with specific primary antibodies, followed by fluorescent secondary antibodies. (A1-C1) Schematic illustration of the respective protein localizations at the pre- or postsynapse. As shown in the merged pictures, Syt 1 co-localized with the active zone marker Munc13-1 (A2), the presynaptic vesicle-bound transporter VGLUT1 (B2) and axonal enriched Ankyrin B (C2), whereas MAP2 visualized the neighboring dendritic compartment. 


\subsubsection{The TCM-evoked increase in AMPAR-mEPSC frequency is not a presynaptic effect}

After validating the presynaptic localization of immunofluorescent Syt1 signals, I performed the Syt1 antibody uptake assay to test the TCM effect on presynaptic vesicle release using primary hippocampal cultures. Throughout the experiment, the voltage-gated sodium channel blocker TTX $(1 \mu \mathrm{M})$ was added to prevent action potential firing in order to exclusively monitor spontaneous vesicle release. As shown in Figure 20, TCM (0.5 mM; $92.17 \pm 6.59 \%)$ did not affect the turnover of synaptic vesicles compared to control (100 $\pm 4.50 \%)$, whereas high potassium chloride concentration evoked an increase in Syt1 signals $(20 \mathrm{mM} \mathrm{KCl} ; 134.59 \pm$ $13.48 \%$ ) [TCM vs. control, $\mathrm{p}>0.05 ; \mathrm{KCl}$ vs. control, $\mathrm{p}=0.014$ ]. High extracellular potassium levels result in increased depolarization of the neuronal cell and were therefore used as a control for the stimulation of presynaptic activity. Interestingly, hypertonic sucrose showed no effect on Syt1 incorporation [0.3 M sucrose; $87.87 \pm 9.61 \%$; sucrose vs. control, $p>0.05$ ], although being reported to trigger vesicle exocytosis from the readily releasable pool and thereby leading to a higher number of mEPSC events (Rosenmund \& Stevens, 1996; Lonart \& Südhof, 2000).

Nevertheless, since TCM exhibited no effect on synaptic vesicle turnover, the TCM-evoked increase in mEPSC frequency is unlikely to result from presynaptic effects on spontaneous vesicle fusion. Thus, the effect is most likely originated at the postsynapse, where TCM might recruit additional transmission sites such as "permanently desensitized" AMPARs which had been inactive before and whose underlying mechanism remains to be investigated. 
A
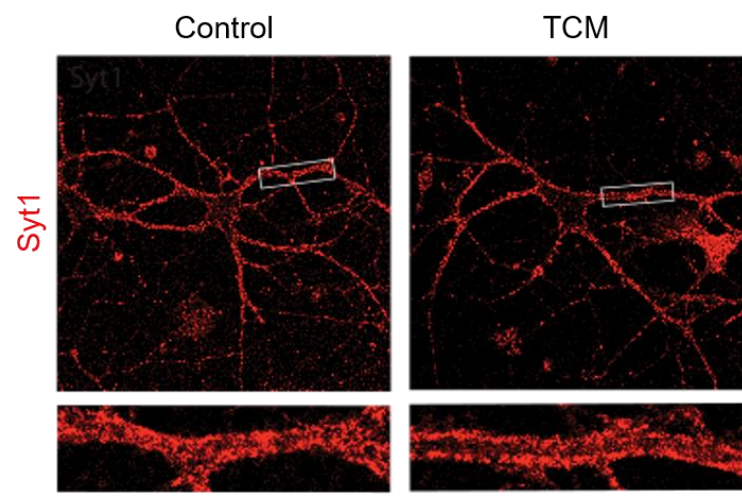

B

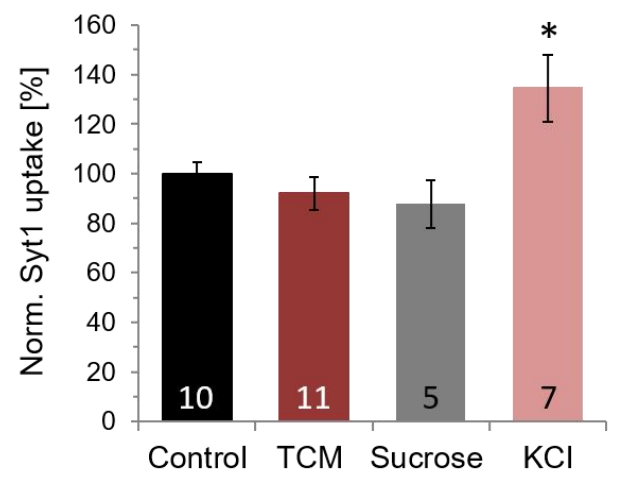

Sucrose
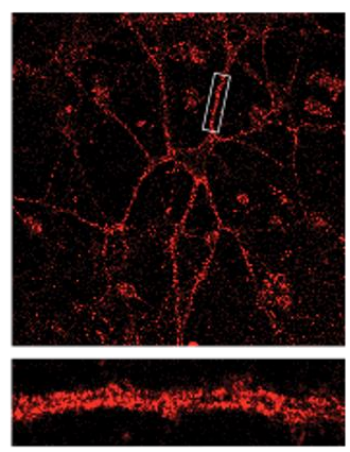
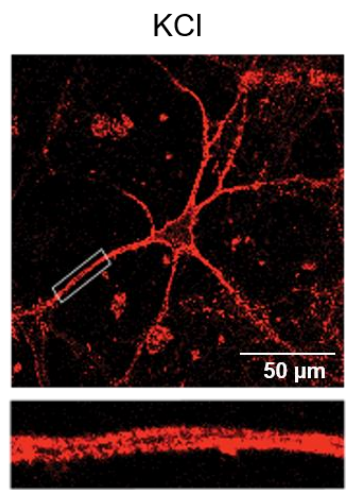

C

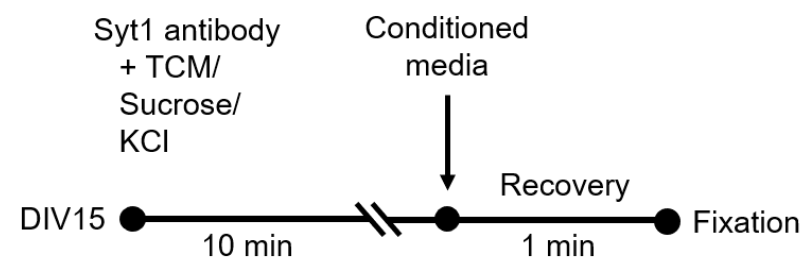

Figure 20: Synaptotagmin 1 uptake assay did not reveal an effect of TCM on presynaptic activity. (A) Dissociated hippocampal culture prepared from PO WT mice was applied to Syt1 uptake assay at 15 DIV. The resulting Syt1 fluorescence signals are displayed for control samples, treatment with the desensitization blocker trichlormethiazide (TCM; $0.5 \mathrm{mM})$, sucrose treatment $(0.3 \mathrm{M})$ and with high concentrations of potassium chloride $(\mathrm{KCl} ; 20 \mathrm{mM})$ as indicated. Throughout all conditions, TTX $(1 \mu \mathrm{M})$ was supplemented to prevent action potentials in order to exclusively monitor spontaneous vesicle fusions. (B) Bar graphs illustrating the amount of Syt1 uptake as normalized to WT with respective $\mathrm{n}$ numbers indicated. Only high potassium chloride concentrations enhanced the presynaptic vesicle turnover. Two-tailed, unpaired Student's t-test. ${ }^{*} p<0.05$. Data were given as means \pm SEM. (C) Schematic illustration of the performed Syt 1 uptake assay with 10 minutes of drug incubation, recovery for 1 minute in the conditioned cell culture medium, followed by fixation and immunostaining. 



\section{DISCUSSION}

\subsection{Knockout of PSD-95 leads to reduced excitatory synaptic strength}

In the present study, I aimed at investigating the function of the DLG-MAGUKs PSD-95 and PSD-93 in basal synaptic transmission by performing fEPSP recordings in hippocampal slices of adult PSD-95 KO, PSD-93 KO and PSD-93/95 DKO mice.

The absence of either PSD-95 alone or both PSD-95 and PSD-93 substantially reduced the strength of synaptic transmission as indicated by shifted input-output curves (Figure 4A). In contrast, loss of PSD-93 alone had no effect, with input-output curves being undistinguishable from WT (Figure 4A). The paired-pulse ratio (PPR) again showed changes in PSD-95 KO and DKO mice, but not in PSD-93 KO mice. Specifically, PPR was reduced in PSD-95 KO and DKO mice, indicating higher probability of presynaptic vesicle release ( $P r)$ (Figure 4B). Since both - synaptic strength and $\mathrm{Pr}$ - remained unchanged upon PSD-93 KO, the decrease in strength and increase in Probserved in DKO mice were most likely mediated by the absence of PSD-95 alone. Collectively, these results clearly demonstrate that loss of PSD-95 influences basal synaptic transmission by reducing its strength and enhancing the Pr. Given the stargazin-mediated interaction of PSD-95 with AMPARs (Fukata et al., 2005) and its direct interaction with NMDARs (Kornau et al., 1995), PSD-95 could possibly regulate synaptic transmission via both glutamate receptors. On one side, PSD-95 could tether AMPARs at the synaptic membrane and on the other side, PSD-95 might regulate AMPAR trafficking through NMDAR-dependent signaling cascades as it has been shown for synaptic plasticity (Xu et al., 2008).

In support of the unchanged basal synaptic transmission in PSD-93 KO mice seen here, previous studies showed that genetic deletion of PSD-93 did not affect synaptic transmission in hippocampus (Elias et al., 2006) and cerebellum (McGee et al., 2001). In contrast, findings for PSD-95 KO mice are puzzling. While some studies found unaltered excitatory synaptic transmission in adult PSD-95 KO mice (Migaud et al., 1998; Elias et al., 2006), others showed substantially reduced synaptic transmission in young (Béïque et al., 2006) and in adult PSD95 deficient mice (Carlisle et al., 2008). In support of reduced excitatory synaptic strength, further studies demonstrated $\sim 50 \%$ reduced AMPAR-mediated EPSCs upon KD of PSD-95 (Nakagawa et al., 2004; Elias et al., 2006; Schlüter et al., 2006). Hence, the majority of studies demonstrates diminished strength of synaptic transmission due to loss of PSD-95, which is also consistent with the present data. While some authors postulated that PSD-95 functions to 
anchor AMPARs at synaptic sites (Schnell et al., 2002; Ehrlich \& Malinow, 2004), Huang et al. (2015a) have linked PSD-95 to experience-dependent maturation of silent synapses. Specifically, PSD-95 KO mice maintain $~ 50 \%$ AMPAR-silent synapses into adulthood in all cortical areas investigated so far, including hippocampus, nucleus accumbens, V1 and medial prefrontal cortex (D. Favaro et al., in press; Huang et al., 2015a; Shukla et al., 2017). Notably, the high number of silent synapses can even be reinstated by acute KD of PSD-95 in V1 of adult mice (Huang et al., 2015a). The function of PSD-95 in silent synapse maturation has additionally been indicated by other studies showing that manipulations of PSD-95 levels mainly affects mEPSC frequency, but not the amplitude, thus indicating selective changes in the number of AMPAR-positive synapses (Béïque \& Andrade, 2003; Stein et al., 2003; Béïque et al., 2006; Carlisle et al., 2008). Given that silent synapses do not transmit at resting potential, the reduced strength of synaptic transmission in PSD-95 KO mice as detected in the present study via fEPSP recordings most likely resulted from high levels of silent synapses. As silent synapses are thought to mature in an LTP-like manner (Liao et al., 1995; Durand et al., 1996; Isaac \& Crair, 1997), PSD-95 presumably couples NMDAR activation to downstream cascades involved in silent synapse maturation.

Conversely, silent synapse maturation has been shown to be accelerated in the absence of PSD-93, resulting in an adult-like state of $<10 \%$ silent synapses in the hippocampus at P20 (D. Favaro et al., in press). By the age of $\sim P 60$, as analyzed in the present study, hippocampal synapses in WT mice are probably also matured, thus showing similar levels as PSD-93 KO mice (Huang et al., 2015a). Hence, the unchanged strength of basal synaptic transmission in PSD-93 KO mice is most likely due to comparable low levels of silent synapses.

The impaired synaptic transmission in PSD-93/95 DKO mice (Figure 4A) is consistent with Elias et al. (2006), also demonstrating a shift in the input-output curve. In consideration of decreased synaptic AMPAR levels in PSD-enriched fractions and reduced mEPSC frequency in the hippocampus of P30-P40 DKO mice (Elias et al., 2006), these findings possibly indicate enhanced silent synapse levels in DKO mice. Contrary to this view, silent synapse levels in V1 of DKO mice have been shown to be unchanged until late critical period (P28) (D. Favaro et al., in press). However, this result does not rule out that the level of silent synapses differs from WT at later developmental stages in a different brain region (i.e. hippocampus) of DKO mice. Excitatory synapses in DKO mice may also differ in other respects, especially by considering the mechanistical difference in silent synapse maturation between DKO and WT mice. Specifically, while silent synapses matured with similar pace in WT and DKO mice until P28, maturation in DKO mice was not anymore regulated by visual experience (D. Favaro et al., in press), possibly resulting in unfavorable connection patterns. Consistent with this notion, DKO mice displayed severely impaired visual acuity (D. Favaro et al., in press). In addition, quantal size was reduced in DKO mice as indicated by reduced mEPSC amplitudes (D. 
Favaro et al., in press), which likely also account for diminished strength of synaptic transmission observed here.

Similar to the altered strength of synaptic transmission in PSD-95 KO and DKO mice, both genotypes exhibited changes in release probability, i.e. reduced PPR und thus higher $\operatorname{Pr}$, while PPR in PSD-93 KO mice was unchanged (Figure 4B). Such a higher $\operatorname{Pr}$ at AMPARtransmitting synapses, as measured through fEPSP recordings in the present study, likely enhances mEPSC frequency and therefore likely leads to an overestimation of the number of AMPAR-positive synapses derived from mEPSC recordings. Thus, the $\sim 50 \%$ reduction in mEPSC frequency reported by Béïque et al. (2006) might be even stronger without such a presynaptic effect being present. This would indicate that numbers of silent synapses might be underestimated in PSD-95 KO mice and would support the more than $50 \%$ reduction in $\mathrm{fEPSP}$ slope in PSD-95 KO mice (Figure 4A). Given that MAGUKs are mainly located at the postsynapse and that maturation of silent synapses is further thought to occur postsynaptically (Liao et al., 1995, 2001; Malinow \& Malenka, 2002), changes in $\operatorname{Pr}$ might be caused by retrograde signaling to the presynaptic terminal (Futai et al., 2007). Accordingly, MAGUKs could affect $\operatorname{Pr}$ via their interaction with neuroligin (Irie et al., 1997), which can in turn bind to presynaptic $\beta$-neurexin through its extracellular domain (Ichtchenko et al., 1995). Such coupling with $\beta$-neurexin could induce changes to the vesicle release machinery (Dean \& Dresbach, 2006).

However, the following comparison with previous studies is conflicting and likely results from the use of different mouse lines and/or different developmental states, since PPR has been shown to be age-dependent (Chavis \& Westbrook, 2001). While the unchanged PPR in PSD$93 \mathrm{KO}$ mice is consistent with D. Favaro et al. (in press) using the same mouse line, Carlisle et al. (2008) reported an increase in PPR in the hippocampus of adult PSD-93 KO mice. Moreover, PPR in DKO mice was found to be unchanged in hippocampus by the age of P30 40 (Elias et al., 2006). Surprisingly, even an enhanced PPR in PSD-95 KO mice has been reported before (Migaud et al., 1998; Carlisle et al., 2008). Since most of the mentioned studies performed fEPSP recordings like I did, results mainly rely on AMPAR-mediated responses, but are also influenced by NMDAR-mediated currents. Given that silent synapses do not transmit at resting potential, they just get activated and thus detected upon membrane depolarization, e.g. resulting from adjacent AMPAR-positive synapses. Thereby, the obtained PPR mainly characterizes presynaptic terminals of mature, AMPAR-transmitting synapses. By considering a rather immature neuronal network in PSD-95 KO and DKO mice, higher $\mathrm{Pr}$ might result from the "attempt" of the network to compensate for the reduced synaptic strength in order to be able to transmit information. Interestingly, previous studies proposed a higher $\operatorname{Pr}$ for silent synapses by separate analysis of AMPAR- and NMDAR-PPRs. Specifically, high $\operatorname{Pr}$ during early synapse development - when silent synapse levels are high - and a subsequent 
gradual decrease in Pr have been reported for hippocampus (Bolshakov \& Siegelbaum, 1995; Chavis \& Westbrook, 2001) and thalamocortex (Yanagisawa et al., 2004). As stated earlier, the here analyzed fEPSP responses are likely to mainly display AMPAR-mediated currents. However, silent synapses primarily contain GluN2B-NMDARs (Béïque et al., 2006; Huang et al., 2009; Brown et al., 2011), which have slower decay kinetics (Cull-Candy \& Leszkiewicz, 2004) and are thus able to integrate synaptic currents across broader time intervals. Therefore, it is possible that GluN2B-NMDARs in silent synapses are more easily activated and thus stronger contribute to the higher Pras detected in the absence of PSD-95.

\subsection{Partly altered synaptic composition in MAGUK KO mice}

The development of excitatory synapses can be subdivided into two phases: synaptogenesis, followed by synaptic maturation. Synaptogenesis involves formation of cell-to-cell contacts and recruitment of synaptic proteins required for synaptic transmission such as AMPARs and NMDARs (Waites et al., 2005). Throughout subsequent synapse maturation, the strength of AMPAR-mediated transmission increases due to gradual AMPAR incorporation, and the NMDAR subunit composition changes from primarily GluN2B- to GluN2A-containing NMDARs (Monyer et al., 1994; Sheng et al., 1994; Wu et al., 1996; Petralia et al., 1999). As excitatory synapse maturation is impaired in PSD-95 KO mice, which are maintaining high levels of silent synapses into adulthood (Huang et al., 2015a), I aimed at examining whether such impaired maturation is linked to altered expression levels of AMPARs and NMDARs.

\subsubsection{Evidence for extrasynaptic AMPARs in silent synapses}

In order to investigate the molecular composition of AMPAR-silent synapses, I analyzed total and surface GluA1 and GluA2 levels of cortical slices in WT and PSD-95 KO mice. Surface levels ranged from $\sim 60-70 \%$ and were comparable with previously reported levels in hippocampal cultures (i.e. $60-70 \%$ of GluA1 and $40-50 \%$ of GluA2) (Hall \& Soderling, 1997). While total expression of GluA1 and GluA2 was unchanged in the absence of PSD-95 (Figure 14C), GluA1 (but not GluA2) surface levels were slightly (7-10\%) reduced at P30 (Figure 17A). Taken together, these data show that loss of PSD-95 has, if any, only minimal effects on AMPAR expression levels. Thus, this effect cannot account for the difference of $\sim 50 \%$ vs. $\sim 25 \%$ silent synapses in PSD-95 KO and WT mice, respectively, observed at this age (Huang et al., 2015a). Therefore, these data indicate that the AMPAR number in silent synapses and (mostly) mature AMPAR-transmitting synapses is highly comparable. Hence, silent synapses might contain inactivated or extrasynaptic AMPARs, resulting in abolished AMPARtransmission. By additionally taking into account the reported reduction of $\sim 50 \%$ in GluA1 and 
GluA2 levels in the core PSD fraction of PSD-95 KO mice (Bonnet et al., 2013; Huang et al., 2015a), AMPARs in silent synapses might rather be located extrasynaptically, since synaptic levels are reduced, whereas total levels, including those at extrasynaptic sites, are not or only minimally affected. Together, these findings support the role of PSD-95 in silent synapse maturation, rather than in clustering AMPARs at the neuronal surface in general.

Analysis of the developmental profile of AMPAR expression from P13 to P30 in WT mice revealed different mechanisms of AMPAR maintenance and turnover between subunits GluA1 and GluA2. While total GluA1 levels showed an increase of $~ 20 \%$, GluA2 levels remained constant (Figure 14C). Conversely, surface expression of GluA1 did not change, while GluA2 surface levels increased by $\sim 20 \%$ (Figure 17A). Collectively, a net increase of both subunits by $20 \%$ from P13 to P30 was detected. These results indicate that, regarding GluA1, total protein expression increases during development while maintaining a constant ratio of intra- to extracellular amount. In contrast, total GluA2 is expressed in a relatively stable manner (with a peak at P20), but progressively gets incorporated into the surface membrane during neuronal development. Hence, transport of GluA1 to the surface membrane at earlier developmental stages seems to be more efficient as compared to GluA2 subunits.

In V1, a developmental increase in total levels of both AMPAR subunits has been reported earlier (Huang et al., 2015a). Specifically, GluA1 levels increased by 15\% between P14 and P30, which is highly comparable to the results obtained from whole cortex samples, i.e. an increase of $20 \%$. In contrast, while total GluA2 levels remained constant in whole cortex and just increased in terms of surface expression, total GluA2 increased by $\sim 40 \%$ in V1. This discrepant data most likely results from differences in the time course of area-specific critical periods (Hensch, 2004), which all contribute to the results obtained from whole cortex. For example, maturation of the somatosensory system through tactile stimulation probably occurs earlier in life than V1 maturation, which is triggered only after eye opening ( P13). Thus, rather drastic changes at the level of individual cortical circuits might have been masked by using whole cortex homogenates due to asynchronous synapse maturation in different sensory systems. To enable more detailed comparison between the two studies, information about the developmental change in surface levels of AMPARs in V1 are needed. In addition, the biological material differed: While Huang et al. (2015a) used P2 crude synaptosomal fractions, I used whole cortex homogenates. 


\subsubsection{Impaired GluN2B to GluN2A switch in PSD-95 KO mice}

Further analysis of the cortical brain samples revealed differences in NMDAR expression by comparing WT with PSD-95 KO mice. Regarding GluN2A, I detected a developmental increase in cortical expression but constant surface levels between P13 and P30, even in the absence of PSD-95 (Figure 14B, Figure 17B). This increase likely results from growth and linkage of new excitatory synapses during early development. Interestingly, GluN2A surface levels displayed a minimum in P20 WT mice, which was absent in PSD-95 KO mice (Figure 17B). The detected variation might be due to the dynamic process of silent synapse maturation, which is impaired in PSD-95 KO mice. However, it needs further investigation and higher sample size in order to confirm this finding.

In contrast to the increase in GluN2A expression, GluN2B levels decreased in WT mice, but remained constant in the absence of PSD-95 (Figure 14B). Analysis of surface GluN2B expression did not reveal any changes when comparing P13 with P30 levels (Figure 17B). Together, these data indicate that loss of PSD-95 impairs the developmental switch of GluN2B- to GluN2A-containing NMDARs. Due to the fact that this subunit switch occurs during early development (Monyer et al., 1994; Sheng et al., 1994) and thus in parallel to silent synapse maturation, such a switch lacking in PSD-95 KO mice is consistent with impaired silent synapse maturation in these mice (Huang et al., 2015a). This finding is supported by a previous study in young (<P25) PSD-95 KO mice, which showed greater contribution of GluN2B-containing receptors to the NMDAR-mediated synaptic current, thereby also indicating an impaired (or delayed) subunit switch (Béïque et al., 2006). On the contrary, a study on cortical PSD fractions reported comparable levels of synaptic GluN2B between adult WT and PSD-95 KO mice (Bonnet et al., 2013). Since I detected a difference in the developmental profile of total GluN2B, but not by comparing absolute levels at $\sim P 30$, the difference between genotypes might biochemically just be detectable by investigation of the developmental profile. Furthermore, the age of the analyzed mice strongly differs between the two studies (P13-P30 in the present study vs. adult, >P60 (Bonnet et al., 2013)).

Similar deficits in the GluN2A/2B subunit switch seen here in PSD-95 KO mice, have also been reported in PSD-93/95 DKO mice (at the age of P30-P40) (Elias et al., 2008). Interestingly, they were shown to be fully rescued by the expression of PSD-95. These findings are partly in line with the somewhat dysfunctional silent synapse maturation in DKO mice (D. Favaro et al., in press), possibly lacking NMDAR subunit switch and therefore not reaching a fully functional state. Rescue of the subunit switch in DKO mice by PSD-95 expression (Elias et al., 2008) clearly points towards a PSD-95-dependent mechanism accounting for the switch. In contrast, other studies indicated a mechanism rather dependent on silent synapse maturation, as dark-rearing of WT mice, which prevents silent synapse maturation, also prevented the GluN2B/2A switch in V1 (although PSD-95 is expressed) (D. 
Favaro et al., in press.; Quinlan et al., 1999). Indeed, dark-rearing maintains high levels of silent synapses by depriving mice of visual experience, which normally triggers silent synapse maturation during critical period plasticity. Importantly, while silent synapse maturation in DKO mice follows the same developmental trajectory as in WT mice, it is not anymore regulated by visual experience (D. Favaro et al., in press). This might indicate that specifically experiencedependent synapse maturation requires a switch from GluN2B to GluN2A expression.

\subsubsection{Unchanged inhibitory tone in PSD-95 KO mice}

The $\mathrm{GABA}_{\alpha 1}$ subunit is particularly enriched at the postsynaptic site of inhibitory synapses formed by parvalbumin-positive (PV+) interneurons on pyramidal cells (Klausberger et al., 2002). While $\mathrm{GABA}_{\alpha 1}$ levels were maintained at a constant extra- to intracellular ratio, as evidenced by largely stable surface levels (Figure 17C), total expression strongly increased by $\sim 50 \%$ between P13 and P30 (Figure 14A), showing the steepest developmental rise in both WT and PSD-95 KO mice compared to all other receptor subunits assessed. This indicates that membrane expression of $\mathrm{GABA}_{\alpha 1}$ receptors strongly increases during the critical period. Interestingly, this process is not affected by loss of PSD-95.

These data are consistent with a study by Huang et al. (2015a), showing a developmental increase in V1 but unaltered levels of GABA ${ }_{\alpha 1}$ and gephyrin - a scaffolding protein of inhibitory synapses - in V1 PSD fractions of PSD-95 KO mice (Huang et al., 2015a). Consistently, developmental maturation ( P28 to P65) of the inhibitory tone of PV+ interneurons onto pyramidal neurons as measured by patch-clamp recordings was similar between WT and PSD-95 KO, indicating that GABAergic inhibition is not changed in adult PSD-95 KO mice (Huang et al., 2015a). These results are at odds with the prevailing view that critical period plasticity is mainly regulated by the local inhibitory tone (Hensch, 2005; Levelt \& Hübener, 2012). Specifically, it is thought that cortical disinhibition enables ocular dominance (OD) plasticity following monocular deprivation (Wu \& Sun, 2015; Sun et al., 2016). Accordingly, loss of excitatory inputs to $\mathrm{PV}+$ interneurons would lead to decreased inhibition, thereby enabling excitatory OD plasticity. Given that PSD-95 KO mice maintain juvenile-like OD plasticity into adulthood (Huang et al., 2015a) and do not show decreased GABA a1 $_{1}$ levels as presented here, or decreased inhibitory tone as reported earlier (Huang et al., 2015a), the model of regulating critical period plasticity has to be refreshed by assigning an important role to PSD-95, in addition to inhibitory interneurons.

Total and surface GABA a1 $_{1}$ even showed slightly higher levels in PSD-95 KO mice as compared to WT at the age of three weeks (P20-21) (Figure 14A and Figure 17C). This possibly indicates some kind of transient compensatory regulation, which is absent by reaching P30. Since pharmacological increase of the GABAergic tone in adult PSD-95 KO mice did not abolish OD plasticity as seen in WT mice (following 7 days of monocular 
deprivation) (Huang et al., 2015a), the transiently increased GABA a1 $_{1}$ level probably do not affect the maintenance of juvenile-like plasticity, possibly because of being cancelled out by the high level of silent synapses. Further investigation by measuring the inhibitory tone in pyramidal neurons of WT and PSD-95 KO mice at the age of P20 could clarify if the difference in $\mathrm{GABA}_{\alpha 1}$ expression leads to altered inhibitory input.

\subsubsection{Homeostasis of spontaneous synaptic transmission is primarily a postsynaptic mechanism}

Despite the developmental maturation of silent synapses into AMPAR-transmitting ones, mEPSC frequency - which is thought to be directly proportional to silent synapse number if the vesicle fusion propensity is unchanged - remains stable in WT mice (Supporting Figure 21 A, appendix and Han et al., 2017). PSD-95 KO mice display a similar developmental profile, but have been shown to maintain $~ 50 \%$ silent synapses after eye opening (Huang et al., 2015a). Hence, the constant level of mEPSC frequency after eye opening was expected in the absence of PSD-95, but unexpected in WT. The similarity of WT and PSD-95 KO trajectories indicates that AMPAR transmission is homeostatically maintained on a stable level during silent synapse maturation in the WT brain. In support of that view, the AMPAR desensitization blocker TCM increases mEPSC frequency in P30 WT mice more than three-fold, while this effect is reduced in an earlier developmental stage (i.e. at P16) (Supporting Figure 21B, appendix), leading to the hypothesis that some AMPAR transmission sites get "permanently desensitized" and thus locked during development, but can be released again by TCM. These sites most likely do not belong to silent synapses, since application of cyclothiazide, which is an AMPAR desensitization blocker similar to TCM (Mitchell \& Fleck, 2007), did not reveal any AMPAR-mediated responses in silent synapses (Montgomery et al., 2001). As it is not clear yet how receptors at these sites are maintained in an inactive state, they will be referred to as "idle sites" in the following.

In idle sites, AMPARs might be desensitized by glutamate, e.g. due to glutamate spillover from adjacent terminals (Kullmann \& Asztely, 1998). In turn, TCM application could block the desensitization, leading to activation of these idle sites. On the other hand, TCM could act at the presynapse by increasing $\operatorname{Pr}$. The presynaptic vesicle population has been reported to be heterogenous and consist of two distinct pools: An evoked and a spontaneous releasable pool (Nosyreva et al., 2013; Crawford \& Kavalali, 2015). While these pools usually interact with each other through vesicular, cytosolic and plasma membrane proteins (Crawford \& Kavalali, 2015), this crosstalk might be impaired in idle sites (or idle synapses), leading to an absence of spontaneous transmitter release. Thus, a conceivable mechanism of TCM might be to unlock the crosstalk between these two vesicle pools. However, by using the Syt1 uptake 
assay, I could rule out that TCM affects presynaptic vesicle release due to an absent effect of TCM on Syt1 incorporation (Figure 20). This result indicates that the homeostasis of spontaneous synaptic transmission is likely to be regulated by a postsynaptic mechanism.

While performing the Syt1 uptake assay, I obtained a rather unexpected result for the effect of high sucrose solution. Whereas high extracellular potassium levels increased vesicle turnover as expected, hyperosmotic sucrose $(0.3 \mathrm{M})$ did not. This is at odds to previous studies reporting that 0.5 molar sucrose solution triggers vesicle exocytosis from the readily releasable pool (Rosenmund \& Stevens, 1996; Lonart \& Südhof, 2000). However, differences due to varying sucrose concentrations cannot be excluded ( $0.3 \mathrm{M}$ vs. $0.5 \mathrm{M})$. Importantly, sucrose $(0.45 \mathrm{M})$ has also been reported to inhibit clathrin-dependent endocytosis by preventing clathrin and adaptors from interacting, which results in empty microcages of "trapped" clathrin (Heuser \& Anderson, 1989; Hansen et al., 1993). This effect could possibly counteract the sucrose-mediated stimulation of spontaneous vesicle release. One might argue that Syt1 in vesicles, which cannot be endocytosed and are kind of stuck at the membrane, would still be detectable by its immunofluorescence. Anyhow, it is likely that, if too many vesicles are stuck, the vesicle recycling machinery gets out of balance and adjusts its activity in form of a negative feedback mechanism. Furthermore, it cannot be excluded that sucrose affects the binding affinity of the Syt1-specific antibody to its target.

The hypothesis of a TCM-mediated increase in spontaneous synaptic transmission at idle sites requires more detailed analysis. However, the present result leads to the conclusion that further investigations should focus on a possible postsynaptic mechanism. Idle sites could account for the homeostatic regulation of AMPAR transmission during progressive silent synapse maturation, thereby providing a balancing function. Hence, the more silent synapses have matured, the more idle sites might exist.

\subsection{Knockout of PSD-95 protects against hypoxia}

The interaction of DLG-MAGUKs with NMDARs is thought to mediate the activation of several downstream signaling pathways in response to $\mathrm{Ca}^{2+}$ influx, thus affecting processes such as synaptic plasticity (Xu et al., 2008). Importantly, both PSD-95 and PSD-93 have been implicated in NMDAR-mediated excitotoxicity, which contributes to neuronal dysfunction and cell damage in diverse neuropathologies including ischemic stroke, Alzheimer's disease and acute brain injury (Dawson \& Dawson, 1998; Lai et al., 2014; Parsons \& Raymond, 2014). However, the specific role of PSD-95 and PSD-93 in this context is elusive and, notably, studies on PSD-95 KO mice are still lacking. In order to shed some light on possibly similar or opposing functions of PSD-95 and PSD-93, I induced excitotoxicity in acute brain slices of the respective KO and DKO mice by hypoxia, leading to spreading depression (HSD). 
Importantly, only the KO of PSD-95 but not PSD-93 provided protection against hypoxia. This finding indicates that only PSD-95 is involved in NMDAR-mediated excitotoxicity following metabolic compromise. Furthermore, simultaneous KO of both PSD-95 and PSD-93 showed partly reduced susceptibility to hypoxia, most likely due to the absence of PSD-95 and not PSD-93. The protective effect of PSD-95 KO was demonstrated by multiple parameters. First of all, the onset of HSD was delayed in absence of PSD-95, as indicated by a delayed HSDaccompanied DC shift (Figure 7A). Second, KO of PSD-95 resulted in a markedly attenuated IOS change, as displayed by reduced tissue light reflectance (Figure 8D). Third, PSD-95 KO mice exhibited a trend towards decelerated HSD wave propagation (Figure 9) and slightly delayed synaptic failure (Figure 11). Finally, the most obvious evidence for reduced susceptibility to hypoxia in PSD-95 deficient mice was their substantially improved recovery of synaptic function (Figure 12A and B). By contrast, single KO of PSD-93 had no effect in all of these parameters. Interestingly, the protective effects in PSD-95 KO mice were consistently abolished by additional KO of PSD-93 in DKO mice, except for improved synaptic recovery. The only parameters which were not altered and therefore did not indicate protection by the absence of PSD-95, were the amplitude and duration of the HSD-associated DC shift (Figure $7 \mathrm{~B} / \mathrm{C}$ ) and the size of affected hippocampal area (Figure 9). Whereas a reduced HSD-invaded area would have been expected, the amplitude and duration of the DC shift depend on the synchronization of underlying neuronal and glial depolarizations, and therefore not necessarily provide evidence for the vulnerability to hypoxia. Specifically, while an improved synchronization could shorten the DC shift, the amplitude would be enhanced. Consistent with the lack of differences in the invaded area between genotypes observed in the present study, no morphological alterations in hippocampal structures of PSD-93 KO and PSD-95 KO mice have been detected so far (Migaud et al., 1998; McGee et al., 2001). Detailed morphological investigations in PSD-93/95 DKO mice are still lacking, but the here presented unchanged DC parameters do not indicate alterations.

The delayed occurrence of HSD (Figure 7A) seen in PSD-95 KO mice might have been influenced by the decreased neuronal excitability (Figure 4A), since neuronal excitability is an important parameter defining the vulnerability of brain tissue to HSD generation (Aitken et al., 1991; Müller, 2000; Müller \& Somjen, 2000b). However, HSD onset was unchanged in DKO mice, which also presented reduced strength of basal synaptic transmission (Figure 4A). Therefore, the protective effect seen in PSD-95 mice is more likely to be genotype-specific, rather than due to reduced excitability.

The here demonstrated protection due to loss of PSD-95 against ischemia-like insults is consistent with several previous studies. Sattler et al. (1999) reported protective effects of antisense RNA-mediated KD of PSD-95 in cortical cultures against NMDAR-dependent excitotoxicity as indicated by reduced cell death after NMDA-treatment. These authors addressed delayed neuronal death, while my results indicate short-term protective effects 
such as delayed HSD onset and improved synaptic recovery. Sattler et al. (1999) furthermore linked the interaction of PSD-95 with NMDARs to toxic NO production, in which PSD-95 serves to couple nNOS-activity to NMDAR-mediated $\mathrm{Ca}^{2+}$ influx. Consequently, following studies used small interfering peptides, which were synthesized to disrupt the NMDAR-PSD95-nNOS interaction and tested them in stroke models. In support of the present data, these so-called "PSD-95 inhibitors" provided protection against ischemic stroke in rodents (Aarts et al., 2002; Sun et al., 2008; Zhou et al., 2010), macaques (Cook et al., 2012a), as well as humans tested in phase II clinical trials, which suffered from fewer infarcts following treatment (Hill et al., 2012). Specifically, the peptides reduced neuronal deficits following MCAO (Aarts et al., 2002; Sun et al., 2008; Zhou et al., 2010), which is comparable to the present study showing improved reinstatement of neuronal function upon hypoxia in PSD-95 KO mice (Figure 12A and B).

In contrast to the unchanged size of the HSD-invaded hippocampal area in PSD-95 KO mice seen here (Figure 9), previous studies, testing PSD-95 inhibitors in MCAO models, reported reduced infarct volumes (Aarts et al., 2002; Sun et al., 2008; Zhou et al., 2010; Cook et al., 2012a). However, using a different lesion model which typically does not result in a penumbra region like MCAO, i.e. photothrombotic stroke, another study did not detect reduced lesion sizes in PSD-95 KO mice (Greifzu et al., 2016). This is consistent with the present data, indicating similarities between impairments induced by photothrombotic stroke and the here addressed hypoxia-induced dysfunctions. Nevertheless, various other parameters including the reduced IOS change and improved functional recovery in PSD-95 KO mice are pointing towards diminished brain damage and an enhanced ability to recover from hypoxia such as in a penumbra region following $\mathrm{MCAO}$, if appropriate treatment is performed (Ferrer \& Planas, 2003). The extent and severity of delayed neuronal damage and/or cell death can, however, not be investigated here, due to the given time-limit of acute brain slices.

There are two main possibilities, which could account for the protective effect in absence of PSD-95. On the one hand, loss of PSD-95 might result in reduced activation of excitotoxicity pathways such as toxic NO production (Sattler et al., 1999). On the other hand, high levels of silent synapses in PSD-95 KO mice (D. Favaro et al., in press; Huang et al., 2015a) may "reinstate" a juvenile, more plastic state, possibly promoting synaptic reorganization following hypoxia. Both possibilities will be further discussed in the following sections (4.4 and 4.5). 


\subsection{Neuronal protection due to high levels of silent synapses?}

Silent synapses represent opportunities for activity- and experience-dependent synaptic connections, therefore consolidating final wiring patterns (Liao et al., 1995; Durand et al., 1996; Itami et al., 2003). Critical periods in early development show especially high levels of neuronal plasticity, enabling sensory cortical networks to adapt to their environment and optimize functional output (Fagiolini \& Hensch, 2000).

In the maturing brain, silent synapse levels gradually decrease during early development (Kerchner \& Nicoll, 2008; Huang et al., 2015a). Thus, neuronal networks lose their state of high plasticity and the ability to rapidly adjust to new experience. Intriguingly, genetic KO of PSD-95 prevented the experience-dependent maturation of silent synapses and maintained high plasticity levels as shown for juvenile-like OD plasticity in V1 (Huang et al., 2015a). Furthermore, acute KD of PSD-95 increased silent synapse levels, facilitates LTP induction (Migaud et al., 1998; Béïque et al., 2006; Carlisle et al., 2008) and reinstates juvenile-like OD plasticity in adult mice (Huang et al., 2015a). Thus, the absence of PSD-95 preserves (or reinstates) a premature, more plastic state of neuronal networks, which is physiologically present only in the "young" brain.

In the present study, a model of transient hypoxia served as a tool to examine the response and vulnerability of neuronal networks to metabolic compromise occurring in diverse neuronal disorders such as ischemic stroke. Ischemic stroke mainly occurs in elderly people and age is known to strongly influence the patients' outcome (Chen et al., 2010; Liu \& McCullough, 2012). In fact, aging emerges as the most important independent risk factor for the incidence and prevalence of stroke with over $80 \%$ of strokes occurring in people aged $>65$ years (Rojas et al., 2007; Rosamond et al., 2008). In line with these epidemiological data, stroke also appears to be age-dependent in diverse animal models (Popa-Wagner et al., 2007; Liu et al., 2009a). Thus, age - or, the maturational synaptic state - plays an important role in susceptibility to stroke and the severity of stroke-induced neuronal impairments. I analyzed the response and neuronal impairments following hypoxia, which is an important aspect of stroke, in WT mice at 2 months of age ( P60) and compared them to age-matched KO mice lacking PSD-93, PSD-95 or both paralogs. Throughout the study, adult PSD-93 KO mice, which contain very low levels of silent synapses (D. Favaro et al., in press), were undistinguishable from WT. Thus, no beneficial or disadvantageous effect on hypoxia-susceptibility due to loss of PSD-93 was found. In contrast, PSD-95 deficiency indeed provided protection in a variety of parameters.

Given the high level of silent synapses in adult PSD-95 KO mice, it is well conceivable that silent synapses account (at least in part) for the detected protection in metabolic compromise. 
Silent synapses might be particularly beneficial for the reinstatement of synaptic function by providing substrates for reorganization of the neuronal network. Comparable effects of PSD95 deficiency have been shown in $\mathrm{V} 1$ as evidenced by preserved OD plasticity after a distant stroke lesion (Greifzu et al., 2016). Since many of the parameters, which indicated protection in PSD-95 KO mice, were unaltered in the DKO, both genotypes lacking PSD-95 were affected differently. Given that high silent synapse levels have been (so far) only detected in PSD-95 KO mice (Huang et al., 2015a), the present results point towards silent synapses being likely important to mediate protection against hypoxia, rather than distracted PSD-95/ NMDAR-mediated excitotoxicity. Whether the PSD-95 inhibitors, which provided protection against ischemic insults in various studies (Aarts et al., 2002; Sun et al., 2008; Zhou et al., 2010; Cook et al., 2012a), also rejuvenates the synapses, as does PSD-95 KD in adult mice (Huang et al., 2015a), is not known yet. However, it is likely that PSD-95-binding to NMDARs and possibly other PDZ-mediated interactions of PSD-95, which might be impaired due to the interfering peptides, are required to maintain a mature synaptic state in the adult brain.

Since DKO mice showed improved synaptic recovery and assuming that silent synapses are an important substrate for the protective effects seen in PSD-95 KO mice, this finding points towards uncomplete synaptic maturation in DKO mice as well. So far, silent synapse levels have only been determined in V1 of younger DKO mice, showing no alterations in the pace of silent synapse maturation, but a distinct mechanism which is experience-independent (D. Favaro et al., in press). Therefore, an increased number of silent synapses in the hippocampus of adult DKO mice cannot be excluded and would be consistent with the reported reduction in mEPSC frequency in the hippocampus of P30-P40 DKO mice, as well as reduced AMPAR levels in PSD-enriched fractions (Elias et al., 2006). Nonetheless, either partly silent as reported for the hippocampus (Elias et al., 2006), or inadequately matured leading to reduced quantal size and partly impaired functional outcome as shown in V1 (D. Favaro et al., in press), these data suggest that synapses lacking both PSD-95 and PSD-93 can facilitate functional recovery following hypoxia.

\subsubsection{CaMKII as a possible target to inhibit silent synapse maturation}

In order to confirm that the protective effect seen in PSD-95 KO (and partly in DKO) mice is mediated by high levels of silent synapses, a possible approach would be to prevent silent synapse maturation independently of PSD-95 deletion. In V1, this can be achieved by darkrearing (Funahashi et al., 2013) or dark-exposure (Guo et al., 2012), but this model is not transferable to the hippocampus. Instead, a molecular mediator for silent synapse maturation has to be addressed and blocked. 
Such a candidate is the $\mathrm{Ca}^{2+}$ /calmodulin-dependent protein kinase II (CaMKII). CaMKII is a signal transduction molecule with developmentally regulated expression and shows specific abundance at the PSD of excitatory synapses (Kennedy et al., 1983; Schulman, 1993). CaMKII has been implicated in synaptogenesis, neuronal growth and is known to be an important player in synaptic plasticity (Soderling, 2000; Lisman et al., 2002). Indeed, inhibition or genetic modification of CaMKII impairs LTP in CA1 hippocampus (Silva et al., 1992; Hinds et al., 1998) and V1 (Kirkwood et al., 1997), most likely by preventing CaMKII-mediated phosphorylation of the AMPAR subunit GluA1 (Barria et al., 1997; Mammen et al., 1997). In line with the broad consensus that silent synapses mature in a LTP-like manner (Liao et al., 1995; Durand et al., 1996), CaMKII has also been implicated in silent synapse maturation. Early studies on retinotectal synapses from Xenopus tadpoles revealed that overexpression of a $\mathrm{Ca}^{2+} /$ calmodulin-independent form of CaMKII mimics silent synapse maturation - as indicated by the appearance of AMPAR-mediated synaptic responses (Wu et al., 1996). Further studies on the visual system of mice identified $\alpha$-CaMKII as an essential kinase for OD plasticity, together with its indispensable ability to autophosphorylate (Gordon \& Stryker, 1996; Taha et al., 2002). Therefore, inhibiting CaMKII activity or its autophosphorylation might prevent - or impair - silent synapse maturation and possibly show similar protective effects against hypoxia-induced metabolic compromise as PSD-95 KO. This would support the idea that decreased susceptibility in PSD-95 KO mice is largely due to the presence of high silent synapse levels.

\subsection{Neuronal protection due to attenuated NMDAR- mediated excitotoxicity?}

Another conceivable reason for the protective effect seen in mice lacking PSD-95 is the disruption of NMDAR-mediated excitotoxicity cascades. Hypothetically, due to loss of PSD-95 as a linker protein, key proteins involved in these cascades get uncoupled from NMDAR overactivation and subsequent $\mathrm{Ca}^{2+}$ overload. Excess of $\mathrm{Ca}^{2+}$ has been shown to trigger diverse toxic downstream pathways including activation of the neuronal NO synthase (nNOS), which has been especially implicated in neuronal disorders such as ischemic stroke (Dawson et al., 1992; Huang et al., 1994; Volbracht et al., 2005). Indeed, inhibition of nNOS activity reduced infarct volumes and neurological deficits in mice subjected to cerebral ischemia (Huang et al., 1994).

As stated earlier, the proposed role of PSD-95 in mediating toxic NO production resulted from studies on neuronal cultures, demonstrating that suppressed PSD-95 expression attenuates NMDAR-mediated NO synthesis as well as excitotoxicity (Sattler et al., 1999). Interestingly, PSD-93 has also been implicated in providing protection against ischemic injury. Specifically, 
PSD-93 deletion also prevented NMDAR/nNOS-dependent toxicity in cortical cultures (Xu et al., 2004; Zhang et al., 2010). Furthermore, KO of PSD-93 in a mouse stroke model resulted in reduced infarct size and diminished neurological deficits (Zhang et al., 2014). However, another study showed that loss of PSD-93 did not provide neuroprotection in the hippocampus of neonatal mice subjected to hypoxia-induced ischemia (Jiang et al., 2003), which is similar to the present study not detecting any protection of PSD-93 KO against hypoxia. Collectively, these studies strongly implicate PSD-95 in stroke-induced neurotoxicity pathways, while the effect of PSD-93 KO needs further investigation.

The structural similarity of PSD-95 and PSD-93 (Cho et al., 1992) and the ability of both to directly interact with NMDARs (Kornau et al., 1995; Niethammer et al., 1996) suggests that both paralogs are capable of mediating NMDAR-dependent excitotoxicity. They interact with NMDARs by binding of their N-terminal PDZ1 and PDZ2 domains to the C-terminal cytoplasmatic tail of GluN2A and GluN2B (Kornau et al., 1995; Niethammer et al., 1996). These first two PDZ repeats are highly conserved throughout all DLG-MAGUK family members, showing 80-90\% amino acid sequence identity (Niethammer et al., 1996). Interaction of PSD-95 or PSD-93 with nNOS has further been shown to be mediated by PDZ2 of the MAGUKs, in turn binding to the PDZ domain of nNOS (Brenman et al., 1996).

\subsubsection{Reduced activity of the NMDAR-nNOS pathway in PSD-95 KO mice?}

Given the structural similarity of PSD-95 and PSD-93, the binding specificity of the PSD-95 inhibitors can be questioned. These small molecules - including Tat-NR2B9c (NA-1), ZL006 and Tat-N-dimer - are assumed to prevent the interaction of PSD-95 with either GluN2B or nNOS (Sun et al., 2008; Zhou et al., 2010; Bach et al., 2012). They have aroused much attention in the past years with one of them (NA-1) having already been tested in a clinical trial of ischemic stroke (Hill et al., 2012).

In addition to stroke-related studies, the same (or similar) interfering molecules have even been used in other contexts of neuronal disorders. Interestingly, two peptides (i.e. IC87201 and Tat-nNOS) - in vitro interfering with the nNOS-PSD-95 interaction - showed protection in mouse models of NMDA-induced acute and chronic pain (Florio et al., 2009). Furthermore, nNOS-PSD-95 interaction has been proposed as a novel target for fear-related disorders such as posttraumatic stress disorder, since the peptide ZL006 attenuated fear memory in the amygdala ( $\mathrm{Li}$ et al., 2018). Despite the well-known involvement of nNOS in mechanisms of depression (Wegener \& Volke, 2010), the same peptide (ZL006) did not induce antidepressant-like effects in rodent depression models (Tillmann et al., 2017). Notably, in a mechanistical in vitro study, ZL006 and IC87201 were unable to interact with the PDZ domains 
of PSD-95 or nNOS, nor inhibited the PDZ-PDZ binding interface of the two proteins (Bach et al., 2015). This is in sharp contrast to the previously reported in vitro interference capability of IC87201 by Florio et al. (2009). Additional doubt on the proposed mechanism of action was generated by a finding of Cui et al. (2007), that Tat-NR2B9c contains higher potency of affecting the interaction of PSD-95 ${ }^{\mathrm{PDZ} 2}$ with GluN2A, rather than with GluN2B. This finding seems surprising, since GluN2A is implicated in pro-survival pathways located the synapse, while extrasynaptic GluN2B mainly mediates excitotoxicity (Hardingham \& Bading, 2003). On top of that, despite the assumed interference of Tat-NR2B9c with GluN2B-PSD-95 interaction (Aarts et al., 2002), the peptide is much more effective at disrupting nNOS-PSD-95 by means

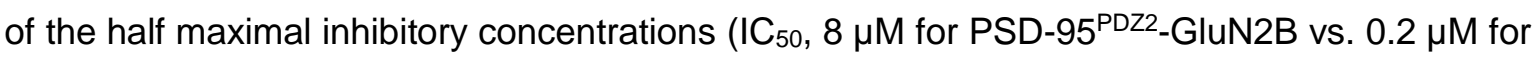

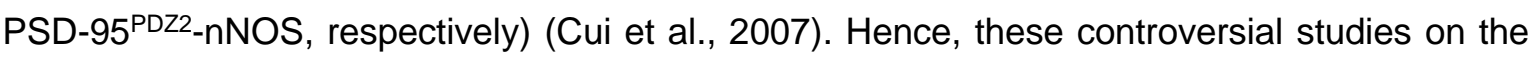
synthetic peptides do not allow any clarification about their in vivo mechanism of action that mediates the protection against ischemic injury.

By taking into account the high structural similarity of PSD-95 and PSD-93 and the reported ability of Tat-NR2B9c to bind both paralogs (Cui et al., 2007), it is likely that the interaction of PSD-93 with GluN2B or nNOS is also affected. Nevertheless, the present study clearly implicates PSD-95 - rather than PSD-93 - in mediating hypoxia-induced excitotoxicity, as evidenced by substantial protection provided by genetic loss of PSD-95 but not PSD-93.

The here obtained results indicate either higher levels of silent synapses or reduced excitotoxicity such as impaired NO production to account for the protective effects seen in PSD-95 KO mice, while both mechanisms do not have to be mutually exclusive. However, the fact that the DKO mice, in which PSD-95-mediated excitotoxicity is abolished as well, showed almost no protection, clearly points towards a key role of high silent synapse levels in PSD-95 $\mathrm{KO}$ mice in providing protection against hypoxia.

A possible approach to further investigate a potential reduction in nNOS activity by PSD-95 deficiency is the targeting of NO substrates. NO (or NO-derived reactive oxygen species) account for the nitration of tyrosine, or nitrosylation of free cysteine residues of diverse proteins (Ischiropoulos, 1998; Lai et al., 2014). Especially the nitrosylation of free cysteine residues (S-nitrosylation), which can arise from broken disulfide bonds due to oxygen deprivation, has been linked to downstream cascades of excitotoxic nNOS activity. In turn, nitrosylation of these proteins activates death signaling or inhibits pro-survival cascades. Reported targets of S-nitrosylation include GAPDH (Hara et al., 2005), the GluR6 subunit of the kainate receptor (Yu et al., 2008) and the src homology 2 domain-containing phosphatase (SHP-2) (Shi et al., 2013). Hence, specific antibodies could be used to detect possible differences in the level of nitration or nitrosylation between WT and PSD-95 KO following the hypoxic event as an indicator for nNOS activity and thus, for excitotoxicity. 


\subsection{How do PSD-95 and PSD-93 mediate different signaling cascades?}

While both PSD-95 and PSD-93 have a specialized role in determining the number of AMPARs at excitatory synapses, they show quite differing roles in synaptic plasticity. PSD-93 deletion enhances the threshold for LTP, but has no influence on LTD (Carlisle et al., 2008). In contrast, PSD-95 counteracts LTP, but promotes LTD (Migaud et al., 1998; Béïque \& Andrade, 2003). Furthermore, the paralogs exhibit opposite functions in cortical network maturation and stabilization during early development (Huang et al., 2015; D. Favaro et al., in press).

The present study adds an additional aspect to this scheme of similar vs. differing or even opposing functions. Specifically, only PSD-95 was found to mediate hypoxia-induced excitotoxicity, as evidenced by lower susceptibility of PSD-95 KO mice. On the contrary, PSD93 deficiency did not provide any protection in the hippocampal tissue. For most parameters, simultaneous KO of PSD-93 even abolished the beneficial effects of PSD-95 deletion as seen in the DKO mice, except for improved recovery of synaptic function. Thus, future studies should further aim at identifying different signaling cascades coupled to NMDAR activation.

\subsubsection{PSD-95 and PSD-93 might be localized at different neuronal sites}

The main question regarding individual functions is, if PSD-95 and PSD-93 are expressed at the same synapses and, if so, if they are located at similar sites. If the paralogs are positioned in high vicinity, they might compete for the same signaling cascade. On the other hand, if they are expressed more distantly (e.g. synaptic vs. peri- or extrasynaptic sites), they might affect different downstream pathways. While earlier studies reported a co-localization of $33 \%$ in the hippocampus (Sans et al., 2000), others demonstrated high levels of co-localization at the same synapse in the cortex (DeGiorgis et al., 2006) and in V1 (D. Favaro et al., in press). Hence, it is most likely that PSD-95 and PSD-93 are expressed at the same synapse, but - so far - their precise localization remains elusive.

It is widely accepted that NMDAR-dependent excitotoxicity relies on extrasynaptic NMDARs, whereas synaptic NMDARs are suggested to have a protective function (Hardingham \& Bading, 2003). Early after birth, most synaptic NMDARs contain GluN2B subunits, which get gradually replaced by GluN2A during synapse maturation (Monyer et al., 1994; Sheng et al., 1994). This subunit switch results in the predominant location of GluN2A at synaptic sites and GluN2B at extrasynaptic sites in matured neurons.

The present study confirms previous observations that the subunit switch is abolished in the 
absence of silent synapse maturation in PSD-95 KO mice (or due to lack of PSD-95 itself) (D. Favaro et al., in press.; Quinlan et al., 1999; Béïque et al., 2006). Thus, PSD-95 lacking mice contain a reduced number of synapses with extrasynaptically located GluN2B-containing NMDARs, which likely mediate neurotoxic cascades. This aspect could provide a link between the two seemingly incoherent ways of lowering susceptibility to hypoxia in PSD-95 deficient mice: (I.) High levels of silent synapses and (II.) reduction of NMDAR-dependent excitotoxic signaling. Furthermore, this study implicates PSD-95 to be located at the extrasynaptic region to higher levels than PSD-93. This hypothesis is supported by a study of Xu et al. (2004), reporting co-localization and complex formation of PSD-93 with GluN2A (not GluN2B) and nNOS in cortical cultures. Hypothetically, PSD-93 rather couples nNOS to synaptic GluN2ANMDARs, resulting in physiological NO production, while PSD-95 mediates extrasynaptic toxic nNOS overactivation.

\subsubsection{Potential interaction partners of PSD-95 and PSD-93}

There have been several attempts to identify unique protein binding partners of either PSD-95 or PSD-93 in order to provide evidence for differentially affected signaling cascades. One candidate is the synaptic GTPase activating protein (SynGAP). SynGAP has been shown to interact with the PDZ-domains of PSD-95 and SAP102 via its C-terminus and to co-locate with PSD-95 and NMDAR in vitro using immunocytochemistry and in vivo via Coimmunoprecipitation (Kim et al., 1998; Rumbaugh et al., 2006). A possible interaction of SynGAP with PSD-93 has not been reported yet. Functionally, SynGAP is involved in Ras kinase activity and subsequent activation of MAPK-mediated pathways such as activity of the pro-survival kinase Erk and the pro-apoptotic kinase p38 (Rumbaugh et al., 2006). Notably, altered expression levels of SynGAP have been detected in PSD-93 KO mice following MCAO-induced ischemia (Rong et al., 2016). Moreover, Rong et al. (2016) showed reduced infarct volumes and neurological deficits in PSD-93 deficient mice, which is in odds to my results lacking any effect in mice of the same genotype. However, as stated before, the interaction and/or co-localization of PSD-93 and SynGAP has not been proved yet. Interestingly, we also found differing phosphorylation levels - indicating a change in activity of SynGAP by comparing total brain extracts of PSD-93 KO and PSD-95 KO mice via a quantitative phospho-tyrosine $(\mathrm{pY})$ proteomic approach (in collaboration with Dr. Wilhelm Haas and Weifeng $\mathrm{Xu}$, Massachusetts, USA). A second candidate protein appearing in both studies with altered expression and phosphorylation levels, respectively, is the protein kinase $C$ (PKC) $\beta$. PKC $\beta$ also acts in $\mathrm{Ca}^{2+}$ signaling pathways and has been shown to associate with GluN1 and GluN2B subunits (Lipp \& Reither, 2011; Rong et al., 2016).

Accordingly, both SynGAP and PKC $\beta$ provide promising targets for further investigation on the 
role of PSD-95 and PSD-93 in mediating signaling specificity due to individual protein binding partners and, presumably, specific neuronal localization. 



\section{BIBLIOGRAPHY}

Aarts M., Liu Y., Liu L., Besshoh S., Arundine M., Gurd J.W., Wang Y.-T., Salter M.W. \& Tymianski M. (2002) Treatment of Ischemic Brain Damage by Perturbing NMDA Receptor- PSD-95 Protein Interactions. Science 298, 846-850.

Abbas A.I., Yadav P.N., Yao W.-D., Arbuckle M.I., Grant S.G.N., Caron M.G. \& Roth B.L. (2009) PSD-95 is essential for hallucinogen and atypical antipsychotic drug actions at serotonin receptors. J Neurosci 29, 7124-36.

Abegg M.H., Savic N., Ehrengruber M.U., McKinney R.A. \& Gähwiler B.H. (2004) Epileptiform activity in rat hippocampus strengthens excitatory synapses. J Physiol 554, 439-48.

Adams D.J., Takeda K. \& Umbach J.A. (1985) Inhibitors of calcium buffering depress evoked transmitter release at the squid giant synapse. J Physio/ 369, 145-59.

Aitken P.G., Fayuk D., Somjen G.G. \& Turner D.A. (1999) Use of Intrinsic Optical Signals to Monitor Physiological Changes in Brain Tissue Slices. Methods 18, 91-103.

Aitken P.G., Jing J., Young J. \& Somjen G.G. (1991) Ion channel involvement in hypoxia-induced spreading depression in hippocampal slices. Brain Res 541, 7-11.

Aitken P.G., Tombaugh G.C., Turner D.A. \& Somjen G.G. (1998) Similar propagation of SD and hypoxic SD-like depolarization in rat hippocampus recorded optically and electrically. $J$ Neurophysiol 80, 1514-21.

Allison D.W., Gelfand V.I., Spector I. \& Craig A.M. (1998) Role of actin in anchoring postsynaptic receptors in cultured hippocampal neurons: differential attachment of NMDA versus AMPA receptors. J Neurosci 18, 2423-36.

Amaral D.G. \& Witter M.P. (1989) The three-dimensional organization of the hippocampal formation: a review of anatomical data. Neuroscience $31,571-91$.

Andrew R.D., Jarvis C.R. \& Obeidat A.S. (1999) Potential Sources of Intrinsic Optical Signals Imaged in Live Brain Slices. Methods 18, 185-196.

Asada K. \& Itakura S. (2012) Social phenotypes of autism spectrum disorders and williams syndrome: similarities and differences. Front Psychol 3, 247.

Bach A., Clausen B.H., Møller M., Vestergaard B., Chi C.N., Round A., Sørensen P.L., Nissen K.B., Kastrup J.S., Gajhede M., Jemth P., Kristensen A.S., Lundström P., Lambertsen K.L. \& Strømgaard K. (2012) A high-affinity, dimeric inhibitor of PSD-95 bivalently interacts with PDZ1-2 and protects against ischemic brain damage. PNAS 109, 3317-22. 
Bach A., Pedersen S.W., Dorr L.A., Vallon G., Ripoche I., Ducki S. \& Lian L.-Y. (2015) Biochemical investigations of the mechanism of action of small molecules ZL006 and IC87201 as potential inhibitors of the nNOS-PDZ/PSD-95-PDZ interactions. Sci Rep 5, 12157.

Bai D., Zhu G., Pennefather P., Jackson M.F., Macdonald J.F. \& Orser B. (2001) Distinct Functional and Pharmacological Properties of Tonic and Quantal Inhibitory Postsynaptic Currents Mediated by-Aminobutyric Acid A Receptors in Hippocampal Neurons. Mol Pharmacol 59, 814-824.

Ballyk B.A., Quackenbush S.J. \& Andrew R.D. (1991) Osmotic effects on the CA1 neuronal population in hippocampal slices with special reference to glucose. J Neurophysio/ 65, 10551066.

Barria A., Muller D., Derkach V., Griffith L.C. \& Soderling T.R. (1997) Regulatory phosphorylation of AMPA-type glutamate receptors by CaM-KII during long-term potentiation. Science 276, 2042-5.

Basarsky T.A., Duffy S.N., Andrew R.D. \& MacVicar B.A. (1998) Imaging spreading depression and associated intracellular calcium waves in brain slices. J Neurosci 18, 7189-99.

Beattie E.C., Carroll R.C., Yu X., Morishita W., Yasuda H., von Zastrow M. \& Malenka R.C. (2000) Regulation of AMPA receptor endocytosis by a signaling mechanism shared with LTD. Nat Neurosci 3, 1291-300.

Béïque J.-C. \& Andrade R. (2003) PSD-95 regulates synaptic transmission and plasticity in rat cerebral cortex. J Physio/ 546, 859-67.

Béique J.-C., Lin D.-T., Kang M.-G., Aizawa H., Takamiya K. \& Huganir R.L. (2006) Synapsespecific regulation of AMPA receptor function by PSD-95. Proc Natl Acad Sci 103, 1953519540.

Ben-Ari Y., Woodin M.A., Sernagor E., Cancedda L., Vinay L., Rivera C., Legendre P., Luhmann H.J., Bordey A., Wenner P., Fukuda A., van den Pol A.N., Gaiarsa J.-L., Cherubini E., Spitzer N.C., San Diego U., Pablo Castillo U.E. \& Einstein A. (2012) Refuting the challenges of the developmental shift of polarity of GABA actions: GABA more exciting than ever! Front Cell Neurosci 6.

Benveniste M. \& Mayer M.L. (1991) Kinetic analysis of antagonist action at N-methyl-D-aspartic acid receptors Two binding sites each for glutamate and glycine. Bioph 59, 560-573.

Berkel S., Marshall C.R., Weiss B., Howe J., Roeth R., Moog U., Endris V., Roberts W., Szatmari P., Pinto D., Bonin M., Riess A., Engels H., Sprengel R., Scherer S.W. \& Rappold G.A. (2010) Mutations in the SHANK2 synaptic scaffolding gene in autism spectrum disorder and mental retardation. Nat Genet 42, 489-91. 
Bienvenu O.J., Wang Y., Shugart Y.Y., Welch J.M., Grados M.A., Fyer A.J., Rauch S.L., McCracken J.T., Rasmussen S.A., Murphy D.L., Cullen B., Valle D., Hoehn-Saric R., Greenberg B.D., Pinto A., Knowles J.A., Piacentini J., Pauls D.L., Liang K.Y., Willour V.L., Riddle M., Samuels J.F., Feng G. \& Nestadt G. (2009) Sapap3 and pathological grooming in humans: Results from the OCD collaborative genetics study. Am J Med Genet 150B, 710-20.

Blednov Y.A., Benavidez J.M., Black M., Leiter C.R., Osterndorff-Kahanek E., Johnson D., Borghese C.M., Hanrahan J.R., Johnston G.A.R., Chebib M. \& Harris R.A. (2014) GABA A Receptors Containing r1 Subunits Contribute to In Vivo Effects of Ethanol in Mice. PLoS One 9, e85525.

Bliss T. V \& Lomo T. (1973) Long-lasting potentiation of synaptic transmission in the dentate area of the anaesthetized rabbit following stimulation of the perforant path. $J$ Physiol 232, 331-356.

Bliss T.V.P. \& Collingridge G.L. (1993) A synaptic model of memory: long-term potentiation in the hippocampus. Nature 361, 31-39.

Boeckers T.M., Bockmann J., Kreutz M.R. \& Gundelfinger E.D. (2002) ProSAP/Shank proteins - a family of higher order organizing molecules of the postsynaptic density with an emerging role in human neurological disease. J Neurochem 81, 903-10.

Bolay H., Reuter U., Dunn A.K., Huang Z., Boas D.A. \& Moskowitz M.A. (2002) Intrinsic brain activity triggers trigeminal meningeal afferents in a migraine model. Nat Med 8, 136-42.

Bolshakov V.Y. \& Siegelbaum S.A. (1995) Regulation of hippocampal transmitter release during development and long-term potentiation. Science 269, 1730-4.

Bonnet S.A.D., Akad D.S., Samaddar T., Liu Y., Huang X., Dong Y. \& Schluter O.M. (2013) Synaptic State-Dependent Functional Interplay between Postsynaptic Density-95 and Synapse-Associated Protein 102. J Neurosci 33, 13398-13409.

Borgdorff A.J. \& Choquet D. (2002) Regulation of AMPA receptor lateral movements. Nature 417, 649-53.

Bortolotto Z.A., Clarke V.R.J., Delany C.M., Parry M.C., Smolders I., Vignes M., Ho K.H., Miu P., Brinton B.T., Fantaske R., Ogden A., Gates M., Ornstein P.L., Lodge D., Bleakman D. \& Collingridge G.L. (1999) Kainate receptors are involved in synaptic plasticity. Nature 402, 297-301.

Boudreau A.C., Milovanovic M., Conrad K.L., Nelson C., Ferrario C.R. \& Wolf M.E. (2012) A protein cross-linking assay for measuring cell surface expression of glutamate receptor subunits in the rodent brain after in vivo treatments. Curr Protoc Neurosci 1, 1-19.

Bredt D.S. \& Nicoll R.A. (2003) AMPA Receptor Trafficking at Excitatory Synapses. Neuron 40, 361-379. 
Brenman J.E., Chao D.S., Gee S.H., Mcgee A.W., Craven S.E., Santillano D.R., Wu Z. \& Huang F. (1996) Interaction of Nitric Oxide Synthase with the Postsynaptic Density Protein PSD-95 and 1-Syntrophin Mediated by PDZ Domains. Cell 84, 757-767.

Brown T.E., Lee B.R., Mu P., Ferguson D., Dietz D., Ohnishi Y.N., Lin Y., Suska A., Ishikawa M., Huang Y.H., Shen H., Kalivas P.W., Sorg B.A., Zukin R.S., Nestler E.J., Dong Y. \& Schlüter O.M. (2011) A silent synapse-based mechanism for cocaine-induced locomotor sensitization. J Neurosci 31, 8163-74.

Brown T.H., Kairiss E.W. \& Keenan C.L. (1990) Hebbian Synapses: Biophysical Mechanisms and Algorithms. Annu Rev Neurosci 13, 475-511.

Burnashev N. \& Rozov A. (2000) Genomic control of receptor function. Cell Mol Life Sci 57, 1499507.

Cai C., Coleman S.K., Niemi K. \& Keinä K. (2002) Selective Binding of Synapse-associated Protein 97 to GluR-A-Amino-5-hydroxy-3-methyl-4-isoxazole Propionate Receptor Subunit Is Determined by a Novel Sequence Motif. J Biol Chem 277, 31484-31490.

Carlisle H.J., Fink A.E., Grant S.G.N. \& O'dell T.J. (2008) Opposing effects of PSD-93 and PSD-95 on long-term potentiation and spike timing-dependent plasticity. J Physiol 586, 5885-5900.

Carroll R.C., Beattie E.C., Xia H., Lü scher C., Altschuler Y., Nicoll R.A., Malenka R.C. \& von Zastrow M. (1999) Dynamin-dependent endocytosis of ionotropic glutamate receptors. PNAS 96, 14112-14117.

Caruana G. \& Bernstein A. (2001) Craniofacial Dysmorphogenesis Including Cleft Palate in Mice with an Insertional Mutation in the discs large Gene. Mol Cell Biol 21, 1475-1483.

Chance B., Cohen P., Jobsis F. \& Schoener B. (1962) Intracellular oxidation-reduction states in vivo. Science 137, 499-508.

Chater T.E. \& Goda Y. (2014) The role of AMPA receptors in postsynaptic mechanisms of synaptic plasticity. Front Cell Neurosci 8, 401.

Chavis P. \& Westbrook G. (2001) Integrins mediate functional pre- and postsynaptic maturation at a hippocampal synapse. Nature 411, 317-21.

Chebabo S.R., Hester M.A., Aitken P.G. \& Somjen G.G. (1995) Hypotonic exposure enhances synaptic transmission and triggers spreading depression in rat hippocampal tissue slices. Brain Res 695, 203-16.

Chen B.-S., Gray J.A., Sanz-Clemente A., Wei Z., Thomas E. V, Nicoll R.A. \& Roche K.W. (2012) SAP102 Mediates Synaptic Clearance of NMDA Receptors. Cell Rep 2, 1120-28.

Chen H.J., Rojas-Soto M., Oguni A. \& Kennedy M.B. (1998) A synaptic Ras-GTPase activating 
protein (p135 SynGAP) inhibited by CaM kinase II. Neuron 20, 895-904.

Chen R.-L., Balami J.S., Esiri M.M., Chen L.-K. \& Buchan A.M. (2010) Ischemic stroke in the elderly: an overview of evidence. Nat Rev Neuro/ 6, 256-65.

Chen Y., Stevens B., Chang J., Milbrandt J., Barres B.A. \& Hell J.W. (2008) NS21: re-defined and modified supplement B27 for neuronal cultures. J Neurosci Methods 171, 239-47.

Cho K.O., Hunt C.A. \& Kennedy M.B. (1992) The rat brain postsynaptic density fraction contains a homolog of the Drosophila discs-large tumor suppressor protein. Neuron 9, 929-42.

Christopherson K.S., Hillier B.J., Lim W.A. \& Bredt D.S. (1999) PSD-95 assembles a ternary complex with the N-methyl-D-aspartic acid receptor and a bivalent neuronal NO synthase PDZ domain. J Biol Chem 274, 27467-73.

Clark G.D., Happel L.T., Zorumski C.F. \& Bazan N.G. (1992) Enhancement of hippocampal excitatory synaptic transmission by platelet-activating factor. Neuron $9,1211-6$.

Clements J.D., Feltz A., Sahara Y. \& Westbrook G.L. (1997) Activation Kinetics of AMPA Receptor Channels Reveal the Number of Functional Agonist Binding Sites. J Neurosci 18, 119-127.

Clements J.D. \& Westbrook G.L. (1991) Activation kinetics reveal the number of glutamate and glycine binding sites on the N-methyl-D-aspartate receptor. Neuron 7, 605-13.

Conn P.J. \& Pin J.-P. (1997) Pharmacology and functions of metabotropic glutamate receptors. Annu Rev Pharmacol Toxicol 37, 205-237.

Contractor A., Swanson G. \& Heinemann S. (2001) Kainate Receptors Are Involved in Short-and Long-Term Plasticity at Mossy Fiber Synapses in the Hippocampus. Neuron 29, 209-16.

Cook D.J., Teves L. \& Tymianski M. (2012a) Treatment of stroke with a PSD-95 inhibitor in the gyrencephalic primate brain. Nature 483, 213-217.

Cook D.J., Teves L. \& Tymianski M. (2012b) A Translational Paradigm for the Preclinical Evaluation of the Stroke Neuroprotectant Tat-NR2B9c in Gyrencephalic Nonhuman Primates. Sci Transl Med 4, 154ra133-154ra133.

Coyle J.T. \& Puttfarcken P. (1993) Oxidative stress, glutamate, and neurodegenerative disorders. Science 262, 689-95.

Crawford D.C. \& Kavalali E.T. (2015) Molecular underpinnings of synaptic vesicle pool heterogeneity. Traffic 16, 338-64.

Crépel V., Hammond C., Chinestra P., Diabira D. \& Ben-Ari Y. (1993) A selective LTP of NMDA receptor-mediated currents induced by anoxia in CA1 hippocampal neurons. J Neurophysiol 70, 2045-55. 
Cui H., Hayashi A., Sun H.-S., Belmares M.P., Cobey C., Phan T., Schweizer J., Salter M.W., Wang Y.T., Tasker R.A., Garman D., Rabinowitz J., Lu P.S. \& Tymianski M. (2007) Neurobiology of Disease PDZ Protein Interactions Underlying NMDA Receptor-Mediated Excitotoxicity and Neuroprotection by PSD-95 Inhibitors. J Neurosci 27, 9901-9915.

Cull-Candy S., Kelly L. \& Farrant M. (2006) Regulation of Ca2+-permeable AMPA receptors: synaptic plasticity and beyond. Curr Opin Neurobiol 16, 288-97.

Cull-Candy S.G. \& Leszkiewicz D.N. (2004) Role of Distinct NMDA Receptor Subtypes at Central Synapses. Sci Signal re16-re16.

Cuthbert P.C., Stanford L.E., Coba M.P., Ainge J.A., Fink A.E., Opazo P., Delgado J.Y., Komiyama N.H., O'dell T.J. \& Grant S.G.N. (2007) Synapse-Associated Protein 102/dlgh3 Couples the NMDA Receptor to Specific Plasticity Pathways and Learning Strategies. J Neurosci 27, 2673-82.

D. Favaro P., Huang X., Hosang L., Stodieck S., Cui L., Schmitz F., Dong Y., Löwel S. \& Schlüter O.M. (in press) An opposing function of paralogs in balancing developmental synapse maturation. PLOS Biol.

Dahlem Y.A., Dahlem M.A., Mair T., Braun K. \& Müller S.C. (2003) Extracellular potassium alters frequency and profile of retinal spreading depression waves. Exp Brain Res 152, 221-228.

Dawson T.M. \& Dawson V.L. (2018) Nitric Oxide Signaling in Neurodegeneration and Cell Death. Adv Pharmacol 82, 57-83.

Dawson T.M., Dawson V.L. \& Snyder S.H. (1992) A novel neuronal messenger molecule in brain: The free radical, nitric oxide. Ann Neurol 32, 297-311.

Dawson V.L. \& Dawson T.M. (1996) Nitric oxide neurotoxicity. J Chem Neuroanat 10, 179-90.

Dawson V.L. \& Dawson T.M. (1998) Nitric oxide in neurodegeneration. Prog Brain Res 118, 21529.

Dawson V.L., Dawson T.M., London E.D., Bredt D.S. \& Snyder S.H. (1991) Nitric oxide mediates glutamate neurotoxicity in primary cortical cultures. Proc Natl Acad Sci U S A 88, 6368-71.

Dean C. \& Dresbach T. (2006) Neuroligins and neurexins: linking cell adhesion, synapse formation and cognitive function. Trends Neurosci 29, 21-9.

DeGiorgis J.A., Galbraith J.A., Dosemeci A., Chen X. \& Reese T.S. (2006) Distribution of the scaffolding proteins PSD-95, PSD-93, and SAP97 in isolated PSDs. Brain Cell Biol 35, 239 250.

Deidda G., Allegra M., Cerri C., Naskar S., Bony G., Zunino G., Bozzi Y., Caleo M. \& Cancedda L. (2015) Early depolarizing GABA controls critical-period plasticity in the rat visual cortex. Nat 
Neurosci 18, 87-96.

Dingledine R., Borges K., Bowie D. \& Traynelis S.F. (1999) The Glutamate Receptor Ion Channels. Pharmacol Rev 51, 7-61.

Dobrunz L.E. \& Stevens C.F. (1997) Heterogeneity of Release Probability, Facilitation, and Depletion at Central Synapses. Neuron 18, 995-1008.

Dohmen C., Sakowitz O.W., Fabricius M., Bosche B., Reithmeier T., Ernestus R.-I., Brinker G., Dreier J.P., Woitzik J., Strong A.J., Graf R. \& Co-Operative Study of Brain Injury Depolarisations (COSBID). (2008) Spreading depolarizations occur in human ischemic stroke with high incidence. Ann Neurol 63, 720-8.

Dreier J.P., Major S., Manning A., Woitzik J., Drenckhahn C., Steinbrink J., Tolias C., OliveiraFerreira A.I., Fabricius M., Hartings J.A., Vajkoczy P., Lauritzen M., Dirnagl U., Bohner G., Strong A.J. \& COSBID study group. (2009) Cortical spreading ischaemia is a novel process involved in ischaemic damage in patients with aneurysmal subarachnoid haemorrhage. Brain 132, 1866-81.

Dudek S.M. \& Bear M.F. (1992) Homosynaptic long-term depression in area CAl of hippocampus and effects of N-methyl-D-aspartate receptor blockade. Neurobiology 89, 4363-4367.

Durand C.M., Betancur C., Boeckers T.M., Bockmann J., Chaste P., Fauchereau F., Nygren G., Rastam M., Gillberg I.C., Anckarsäter H., Sponheim E., Goubran-Botros H., Delorme R., Chabane N., Mouren-Simeoni M.-C., de Mas P., Bieth E., Rogé B., Héron D., Burglen L., Gillberg C., Leboyer M. \& Bourgeron T. (2007) Mutations in the gene encoding the synaptic scaffolding protein SHANK3 are associated with autism spectrum disorders. Nat Genet 39, $25-7$.

Durand G.M., Kovalchuk Y. \& Konnerth A. (1996) Long-term potentiation and functional synapse induction in developing hippocampus. Nature 381, 71-5.

Ehrlich I. \& Malinow R. (2004) Cellular/Molecular Postsynaptic Density 95 controls AMPA Receptor Incorporation during Long-Term Potentiation and Experience-Driven Synaptic Plasticity. $J$ Neurosci 24, 916-927.

El-Husseini A.E., Schnell E., Chetkovich D.M., Nicoll R.A. \& Bredt D.S. (2000) PSD-95 involvement in maturation of excitatory synapses. Science $\mathbf{2 9 0}, 1364-8$.

Elias G.M., Elias L.A.B., Apostolides P.F., Kriegstein A.R. \& Nicoll R.A. (2008) Differential trafficking of AMPA and NMDA receptors by SAP102 and PSD-95 underlies synapse development. PNAS 30, 20953-20958.

Elias G.M., Funke L., Stein V., Grant S.G., Bredt D.S. \& Nicoll R.A. (2006) Synapse-Specific and Developmentally Regulated Targeting of AMPA Receptors by a Family of MAGUK Scaffolding 
Proteins. Neuron 52, 307-320.

Ellis P.D., Bissoon N. \& Gurd J.W. (1988) Synaptic protein tyrosine kinase: partial characterization and identification of endogenous substrates. J Neurochem 51, 611-20.

Engelhardt M., Vorwald S., Sobotzik J.-M., Bennett V. \& Schultz C. (2013) Ankyrin-B structurally defines terminal microdomains of peripheral somatosensory axons. Brain Struct Funct 218, 1005-1016.

Erwin J.A., Paquola A.C.M., Singer T., Gallina I., Novotny M., Quayle C., Bedrosian T., Ivanio F., Butcher C.R., Herdy J.R., Sarkar A., Lasken R.S., Muotri A.R. \& Gage F.H. (2016) L1Associated Genomic Regions are Deleted in Somatic Cells of the Healthy Human Brain. Nat Neurosci 19, 1583-91.

Espinosa J.S. \& Stryker M.P. (2012) Development and Plasticity of the Primary Visual Cortex. Neuron 75, 230-249.

Fabricius M., Fuhr S., Bhatia R., Boutelle M., Hashemi P., Strong A.J. \& Lauritzen M. (2006) Cortical spreading depression and peri-infarct depolarization in acutely injured human cerebral cortex. Brain 129, 778-90.

Fagiolini M. \& Hensch T.K. (2000) Inhibitory threshold for critical-period activation in primary visual cortex. Nature 404, 183-6.

Farrant M. \& Nusser Z. (2005) Variations on an inhibitory theme: phasic and tonic activation of GABAA receptors. Nat Rev Neurosci 6, 215-229.

Feldman D.E. (2009) Synaptic Mechanisms for Plasticity in Neocortex. Annu Rev Neurosci 32, 3355.

Ferrer I. \& Planas A.M. (2003) Signaling of cell death and cell survival following focal cerebral ischemia: life and death struggle in the penumbra. J Neuropathol Exp Neurol 62, 329-39.

Feyder M., Karlsson R.-M., Lyman M., Bock R., Momenan R., Munasinghe J., Scattoni M.L., Ihne J., Camp M., Graybeal C., Strathdee D., Begg A., Alvarez V.A., Kirsch P., Rietschel M., Cichon S., Walter H., Meyer-Lindenberg A., Grant S.G.N. \& Holmes A. (2010) Association of Mouse Dlg4 (PSD-95) Gene Deletion and Human DLG4 Gene Variation With Phenotypes Relevant to Autism Spectrum Disorders and Williams' Syndrome. Am J Psychiatry 167, 15081517.

Fischer M., Reuter J., Gerich F.J., Hildebrandt B., Hagele S., Katschinski D. \& Muller M. (2008) Enhanced Hypoxia Susceptibility in Hippocampal Slices From a Mouse Model of Rett Syndrome. J Neurophysiol 101, 1016-1032.

Florio S.K., Loh C., Huang S.M., Iwamaye A.E., Kitto K.F., Fowler K.W., Treiberg J.A., Hayflick 
J.S., Walker J.M., Fairbanks C.A. \& Lai Y. (2009) Disruption of nNOS-PSD95 protein-protein interaction inhibits acute thermal hyperalgesia and chronic mechanical allodynia in rodents. $\mathrm{Br}$ J Pharmacol 158, 494-506.

Fredj N. Ben \& Burrone J. (2009) A resting pool of vesicles is responsible for spontaneous vesicle fusion at the synapse. Nat Neurosci 12, 751-758.

Frenguelli B.G. (1997) The effects of metabolic stress on glutamate receptor-mediated depolarizations in the in vitro rat hippocampal slice. Neuropharmacology 36, 981-91.

Frerking M., Borges S. \& Wilson M. (1997) Are Some Minis Multiquantal? J Neurophysiol 78, 1293-1304.

Frerking M. \& Nicoll R.A. (2000) Synaptic kainate receptors. Curr Opin Neurobiol 10, 342-351.

Frischknecht R., Heine M., Perrais D., Seidenbecher C.I., Choquet D. \& Gundelfinger E.D. (2009) Brain extracellular matrix affects AMPA receptor lateral mobility and short-term synaptic plasticity. Nat Neurosci 12, 897-904.

Fromer M., Pocklington A.J., Kavanagh D.H., Williams H.J., Dwyer S., Gormley P., Georgieva L., Rees E., Palta P., Ruderfer D.M., Carrera N., Humphreys I., Johnson J.S., Roussos P., Barker D.D., Banks E., Milanova V., Grant S.G., Hannon E., Rose S.A., Chambert K., Mahajan M., Scolnick E.M., Moran J.L., Kirov G., Palotie A., Mccarroll S.A., Holmans P., Sklar P., Owen M.J., Purcell S.M. \& O'donovan M.C. (2014) De novo mutations in schizophrenia implicate synaptic networks. Nature 506, 179-184.

Fukata Y., Tzingounis A. V, Trinidad J.C., Fukata M., Burlingame A.L., Nicoll R.A. \& Bredt D.S. (2005) Molecular constituents of neuronal AMPA receptors. J Cell Biol 169, 399-404.

Funahashi R., Maruyama T., Yoshimura Y. \& Komatsu Y. (2013) Silent synapses persist into adulthood in layer 2/3 pyramidal neurons of visual cortex in dark-reared mice. $J$ Neurophysiol 109, 2064-2076.

Futai K., Kim M.J., Hashikawa T., Scheiffele P., Sheng M., Hayashi Y. \& Author N.N. (2007) Retrograde modulation of presynaptic release probability through signaling mediated by PSD95-neuroligin. Nat Neurosci 10, 186-195.

Gallyas F., Ball S.M. \& Molnar E. (2003) Assembly and cell surface expression of KA-2 subunitcontaining kainate receptors. $J$ Neurochem $86,1414-1427$.

Gardoni F., Ghiglieri V., Di Luca M. \& Calabresi P. (2010) Assemblies of glutamate receptor subunits with post-synaptic density proteins and their alterations in Parkinson's disease. Prog Brain Res 183, 169-82.

Garthwaite J. \& Boulton C.L. (1995) Nitric oxide signaling in the central nervous system. Annu Rev 
Physiol 57, 683-706.

Gauthier J., Champagne N., Lafrenière R.G., Xiong L., Spiegelman D., Brustein E., Lapointe M., Peng H., Côté M., Noreau A., Hamdan F.F. \& Addington A.M. (2010) De novo mutations in the gene encoding the synaptic scaffolding protein SHANK3 in patients ascertained for schizophrenia. PNAS 107, 7863-7868.

Gerich F.J., Hepp S., Probst I. \& Müller M. (2006) Mitochondrial inhibition prior to oxygenwithdrawal facilitates the occurrence of hypoxia-induced spreading depression in rat hippocampal slices. J Neurophysio/ 96, 492-504.

Gido G., Kristián T., Katsura K. \& Siesjö B.K. (1994) The influence of repeated spreading depression-induced calcium transients on neuronal viability in moderately hypoglycemic rats. Exp Brain Res 97, 397-403.

Goebel D.J. \& Poosch M.S. (1999) NMDA receptor subunit gene expression in the rat brain: a quantitative analysis of endogenous mRNA levels of NR1Com, NR2A, NR2B, NR2C, NR2D and NR3A. Mol Brain Res 69, 164-170.

Gomperts S.N. (1996) Clustering membrane proteins: It's all coming together with the PSD95/SAP90 protein family. Cell 84, 659-62.

Gordon J.A. \& Stryker M.P. (1996) Experience-Dependent Plasticity of Binocular Responses in the Primary Visual Cortex of the Mouse. J Neurosci 76, 3274-3286.

Gozlan H., Diabira D., Chinestra P. \& Ben-Ari Y. (1994) Anoxic LTP is mediated by the redox modulatory site of the NMDA receptor. J Neurophysiol 72, 3017-22.

Grafstein B. (1956) Mechanism of spreading cortical depression. J Neurophysiol 19, 154-71.

Greifzu F., Parthier D., Goetze B., Schlüter O.M. \& Löwel S. (2016) Ocular Dominance Plasticity after Stroke Was Preserved in PSD-95 Knockout Mice. PLoS One 11, e0149771.

Groc L., Gustafsson B. \& Hanse E. (2006) AMPA signalling in nascent glutamatergic synapses: there and not there! Trends Neurosci 29, 132-139.

Groc L., Heine M., Cognet L., Brickley K., Stephenson F.A., Lounis B. \& Choquet D. (2004) Differential activity-dependent regulation of the lateral mobilities of AMPA and NMDA receptors. Nat Neurosci 7, 695-6.

Guo Y., Huang S., de Pasquale R., McGehrin K., Lee H.-K., Zhao K. \& Kirkwood A. (2012) Dark exposure extends the integration window for spike-timing-dependent plasticity. $J$ Neurosci 32 , 15027-35.

Hadzic M., Jack A. \& Wahle P. (2017) lonotropic glutamate receptors: Which ones, when, and where in the mammalian neocortex. J Comp Neurol 525, 976-1033. 
Hall R.A. \& Soderling T.R. (1997) Quantitation of AMPA receptor surface expression in cultured hippocampal neurons. Neuroscience 78, 361-71.

Han K.-S., Cooke S.F. \& Xu W. (2017) Experience-Dependent Equilibration of AMPAR-Mediated Synaptic Transmission during the Critical Period. Cell Rep 18, 892-904.

Hansen A.J. \& Zeuthen T. (1981) Extracellular ion concentrations during spreading depression and ischemia in the rat brain cortex. Acta Physiol Scand 113, 437-45.

Hansen S.H., Sandvig K. \& van Deurs B. (1993) Clathrin and HA2 adaptors: effects of potassium depletion, hypertonic medium, and cytosol acidification. J Cell Biol 121, 61-72.

Hara M.R., Agrawal N., Kim S.F., Cascio M.B., Fujimuro M., Ozeki Y., Takahashi M., Cheah J.H., Tankou S.K., Hester L.D., Ferris C.D., Hayward S.D., Snyder S.H. \& Sawa A. (2005) Snitrosylated GAPDH initiates apoptotic cell death by nuclear translocation following Siah1 binding. Nat Cell Biol 7, 665-74.

Hardingham G.E. \& Bading H. (2003) The Yin and Yang of NMDA receptor signalling. Trends Neurosci 26, 81-89.

Hardingham G.E. \& Bading H. (2010) Synaptic versus extrasynaptic NMDA receptor signalling: Implications for neurodegenerative disorders. Nat Rev Neurosci 11, 682-696.

Van Harreveld A. (1978) Two mechanisms for spreading depression in the chicken retina. $J$ Neurobio/ 9, 419-31.

Hayashi-Takagi A., Yagishita S., Nakamura M., Shirai F., Wu Y.I., Loshbaugh A.L., Kuhlman B., Hahn K.M. \& Kasai H. (2015) Labelling and optical erasure of synaptic memory traces in the motor cortex. Nature 525, 333-338.

Hensch T.K. (2004) Critical period regulation. Annu Rev Neurosci 27, 549-79.

Hensch T.K. (2005) Critical period plasticity in local cortical circuits. Nat Rev Neurosci 6, 877-888.

Hepp S., Gerich F.J. \& Müller M. (2005) Sulfhydryl Oxidation Reduces Hippocampal Susceptibility to Hypoxia-Induced Spreading Depression by Activating BK Channels. J Neurophysiol 94, 1091-1103.

Hepp S. \& Müller M. (2008) Sulfhydryl oxidation: A potential strategy to achieve neuroprotection during severe hypoxia? Neuroscience 152, 903-912.

Herreras O. \& Somjen G.G. (1993) Analysis of potential shifts associated with recurrent spreading depression and prolonged unstable spreading depression induced by microdialysis of elevated K+ in hippocampus of anesthetized rats. Brain Res 610, 283-94.

Heuser J.E. \& Anderson R.G. (1989) Hypertonic media inhibit receptor-mediated endocytosis by 
blocking clathrin-coated pit formation. J Cell Biol 108, 389-400.

Hill M.D., Martin R.H., Mikulis D., Wong J.H., Silver F.L., TerBrugge K.G., Milot G., Clark W.M., MacDonald R.L., Kelly M.E., Boulton M., Fleetwood I., McDougall C., Gunnarsson T., Chow M., Lum C., Dodd R., Poublanc J., Krings T., Demchuk A.M., Goyal M., Anderson R., Bishop J., Garman D., Tymianski M. \& ENACT trial investigators. (2012) Safety and efficacy of NA-1 in patients with iatrogenic stroke after endovascular aneurysm repair (ENACT): a phase 2, randomised, double-blind, placebo-controlled trial. Lancet Neurol 11, 942-950.

Hinds H.L., Tonegawa S. \& Malinow R. (1998) CA1 long-term potentiation is diminished but present in hippocampal slices from alpha-CaMKII mutant mice. Learn Mem 5, 344-54.

Hofmeijer J., Michel ; \& Van Putten J.A.M. (2012) Ischemic Cerebral Damage An Appraisal of Synaptic Failure. Stroke 43, 607-615.

Hollmann M. \& Heinemann S. (1994) Cloned Glutamate Receptors. Annu Rev Neurosci 17, 31108.

Hori N. \& Carpenter D.O. (1994) Functional and morphological changes induced by transient in vivo ischemia. Exp Neurol 129, 279-89.

Hossmann K.A. (1994) Glutamate-mediated injury in focal cerebral ischemia: the excitotoxin hypothesis revised. Brain Pathol 4, 23-36.

Hossmann K.A. (1996) Periinfarct depolarizations. Cerebrovasc Brain Metab Rev 8, 195-208.

Howard M.A., Elias G.M., Elias L.A.B., Swat W. \& Nicoll R.A. (2010) The role of SAP97 in synaptic glutamate receptor dynamics. PNAS 107, 3805-3810.

Huang X., Stodieck S.K., Goetze B., Cui L., Wong M.H., Wenzel C., Hosang L., Dong Y., Löwel S. \& Schlüter O.M. (2015a) Progressive maturation of silent synapses governs the duration of a critical period. Proc Natl Acad Sci 112, E3131-E3140.

Huang Y.H., Lin Y., Mu P., Lee B.R., Brown T.E., Wayman G., Marie H., Liu W., Yan Z., Sorg B.A., Schlüter O.M., Zukin R.S. \& Dong Y. (2009) In vivo cocaine experience generates silent synapses. Neuron $63,40-7$.

Huang Y.H., Schlüter O.M. \& Dong Y. (2015b) Silent Synapses Speak Up. Neurosci 21, 451-459.

Huang Z., Huang P.L., Panahian N., Dalkara T., Fishman M.C. \& Moskowitz M.A. (1994) Effects of cerebral ischemia in mice deficient in neuronal nitric oxide synthase. Science $265,1883-5$.

Huang Z.J., Kirkwood A., Pizzorusso T., Porciatti V., Morales B., Bear M.F., Maffei L. \& Tonegawa S. (1999) BDNF regulates the maturation of inhibition and the critical period of plasticity in mouse visual cortex. Cell 98, 739-55. 
Hubel D.H. \& Wiesel T.N. (1970) The period of susceptibility to the physiological effects of unilateral eye closure in kittens. J Physiol 206, 419-436.

Huettner J.E. \& Baughman R.W. (1986) Primary culture of identified neurons from the visual cortex of postnatal rats. J Neurosci 6, 3044-60.

Ichtchenko K., Hata Y., Nguyen T., Uiirich B., Messier M., Moomaw C. \& S0dhof T.C. (1995) Neuroligin 1: A Splice Site-Specific Ligand for I-Neurexins. Cell 81, 435-443.

Irie M., Hata Y., Takeuchi M., Ichtchenko K., Toyoda A., Hirao K., Takai Y., Rosahl T.W. \& Südhof T.C. (1997) Binding of neuroligins to PSD-95. Science 277, 1511-5.

Isaac J.T.R. \& Crair M.C. (1997) Silent Synapses during Development of Thalamocortical Inputs. Neuron 18, 269-280.

Isaac J.T.R., Nicoll R.A. \& Malenka R.C. (1995) Evidence for Silent Synapses: Implications for the Expression of LTP. Neuron 15, 427-434.

Ischiropoulos H. (1998) Biological tyrosine nitration: a pathophysiological function of nitric oxide and reactive oxygen species. Arch Biochem Biophys 356, 1-11.

Itami C., Kimura F., Kohno T., Matsuoka M., Tsumoto T. \& Nakamura S. (2003) Brain-derived neurotrophic factor-dependent unmasking of "silent" synapses in the developing mouse barrel cortex. PNAS 28, 13069-13074.

Jamain S., Quach H., Betancur C., Råstam M., Colineaux C., Gillberg I.C., Soderstrom H., Giros B., Leboyer M., Gillberg C., Bourgeron T. \& Paris Autism Research International Sibpair Study P.A.R.I.S. (PARIS). (2003) Mutations of the X-linked genes encoding neuroligins NLGN3 and NLGN4 are associated with autism. Nat Genet 34, 27-9.

Jiang X., Mu D., Biran V., Faustino J., Chang S., Rincón C.M., Sheldon R.A. \& Ferriero D.M. (2008) Activated Src kinases interact with the $\mathrm{N}$-methyl-D-aspartate receptor after neonatal brain ischemia. Ann Neurol 63, 632-41.

Jiang X., Mu D., Sheldon R.A., Glidden D. V. \& Ferriero D.M. (2003) Neonatal Hypoxia-Ischemia Differentially Upregulates MAGUKs and Associated Proteins in PSD-93-Deficient Mouse Brain. Stroke 34, 2958-2963.

Jöbsis F.F., Keizer J.H., LaManna J.C. \& Rosenthal M. (1977) Reflectance spectrophotometry of cytochrome aa3 in vivo. $J$ Appl Physiol 43, 858-72.

Jones N. (2011) Stroke: Disruption of the nNOS-PSD-95 complex is neuroprotective in models of cerebral ischemia. Nat Rev Neurol 7, 61.

Kandel E.R., Schwartz J.H. (James H. \& Jessell T.M. (2000) Principles of neural science. McGrawHill, Health Professions Division. 
Kaneda M., Farrant M. \& Cull-Candy S.G. (1995) Whole-cell and single-channel currents activated by GABA and glycine in granule cells of the rat cerebellum. J Physiol 485, 419-435.

Katz L.C. \& Shatz C.J. (1996) Synaptic activity and the construction of cortical circuits. Science 274, 1133-8.

Kauer J.A., Malenka R.C. \& Nicoll R.A. (1988) NMDA application potentiates synaptic transmission in the hippocampus. Nature 334, 250-252.

Kawauchi S., Sato S., Ooigawa H., Nawashiro H., Ishihara M. \& Kikuchi M. (2008) Simultaneous measurement of changes in light absorption due to the reduction of cytochrome $c$ oxidase and light scattering in rat brains during loss of tissue viability. Appl Opt 47, 4164-76.

Kelly P.T. \& Cotman C.W. (1978) Synaptic proteins. Characterization of tubulin and actin and identification of a distinct postsynaptic density polypeptide. J Cell Biol 79, 173-83.

Kennedy M., Bennett M. \& Erondu N. (1983) Biochemical and immunochemical evidence that the \&quot;major postsynaptic density protein\&quot; is a subunit of a calmodulin-dependent protein kinase. Proc Natl Acad Sci U S A 80, 7357-61.

Kennedy M.B. (1997) The postsynaptic density at glutamatergic synapses. Trends Neurosci 20, 264-8.

Kennedy M.B. (2000) Signal-processing machines at the postsynaptic density. Science 290, 7504.

Kerchner G.A. \& Nicoll R.A. (2008) Silent synapses and the emergence of a postsynaptic mechanism for LTP. Nat Rev Neurosci 9, 813-25.

Kessels H.W. \& Malinow R. (2009) Synaptic AMPA Receptor Plasticity and Behavior. Neuron 61, 340-350.

Kim J.H., Liao D., Lau L.F. \& Huganir R.L. (1998) SynGAP: a synaptic RasGAP that associates with the PSD-95/SAP90 protein family. Neuron 20,683-691.

Kirkwood A., Silva A. \& Bear M.F. (1997) Age-dependent decrease of synaptic plasticity in the neocortex of alphaCaMKII mutant mice. Proc Natl Acad Sci U S A 94, 3380-3.

Kirov G., Pocklington A.J., Holmans P., Ivanov D., Ikeda M., Ruderfer D., Moran J., Chambert K., Toncheva D., Georgieva L., Grozeva D., Fjodorova M., Wollerton R., Rees E., Nikolov I., Van De Lagemaat L.N., Bayé A.', Fernandez E., Olason P.I., Bö Ttcher Y., Komiyama N.H., Collins M.O., Choudhary J., Stefansson K., Stefansson H., Grant S., Purcell S., Sklar P., O'donovan M.C. \& Owen M.J. (2011) De novo CNV analysis implicates specific abnormalities of postsynaptic signalling complexes in the pathogenesis of schizophrenia. Mol Psychiatry 17, 142-153. 
Kistner U., Wenzel B.M., Veh R.W., Cases-Langhoff C., Garner A.M., Appeltauer U., Voss B., Gundelfinger E.D. \& Garner C.C. (1993) SAP90, a rat presynaptic protein related to the product of the Drosophila tumor suppressor gene dlg-A. J Biol Chem 268, 4580-3.

Klausberger T., Roberts J.D.B. \& Somogyi P. (2002) Cell type- and input-specific differences in the number and subtypes of synaptic GABA(A) receptors in the hippocampus. $J$ Neurosci 22, 2513-21.

Kornau H.C., Schenker L.T., Kennedy M.B. \& Seeburg P.H. (1995) Domain interaction between NMDA receptor subunits and the postsynaptic density protein PSD-95. Science 269, 173740.

Kristiansen L. V, Beneyto M., Haroutunian V. \& Meador-Woodruff J.H. (2006) Changes in NMDA receptor subunits and interacting PSD proteins in dorsolateral prefrontal and anterior cingulate cortex indicate abnormal regional expression in schizophrenia. Mol Psychiatry 11, 737-747.

Krüger H., Heinemann U. \& Luhmann H.J. (1999) Effects of ionotropic glutamate receptor blockade and $5-\mathrm{HT} 1 \mathrm{~A}$ receptor activation on spreading depression in rat neocortical slices. Neuroreport 10, 2651-6.

Krüger J.M., Favaro P.D., Liu M., Kitlinska A., Huang X., Raabe M., Akad D.S., Liu Y., Urlaub H., Dong Y., Xu W. \& Schluter O.M. (2013) Differential Roles of Postsynaptic Density-93 Isoforms in Regulating Synaptic Transmission. J Neurosci 33, 15504-15517.

Kullmann D.M. (2001) Minireview Presynaptic Kainate Receptors in the Hippocampus: Slowly Emerging from Obscurity. Neuron 32, 561-564.

Kullmann D.M. \& Asztely F. (1998) Extrasynaptic glutamate spillover in the hippocampus: evidence and implications. Trends Neurosci 21, 8-14.

Kunimoto M. (1995) A neuron-specific isoform of brain ankyrin, 440-kD ankyrinB, is targeted to the axons of rat cerebellar neurons. $J$ Cell Biol 131, 1821-9.

Lai T.W., Zhang S. \& Wang Y.T. (2014) Excitotoxicity and stroke: Identifying novel targets for neuroprotection. Prog Neurobiol 115, 157-188.

Lau L.F., Mammen A., Ehlers M.D., Kindler S., Chung W.J., Garner C.C. \& Huganir R.L. (1996) Interaction of the $\mathrm{N}$-methyl-D-aspartate receptor complex with a novel synapse-associated protein, SAP102. J Biol Chem 271, 21622-8.

Lauri S.E., Bortolotto Z.A., Bleakman D., Ornstein P.L., Lodge D., Isaac J.T. \& Collingridge G.L. (2001) A Critical Role of a Facilitatory Presynaptic Kainate Receptor in Mossy Fiber LTP. Neuron 32, 697-709. 
Lauritzen M. (1987) Cerebral blood flow in migraine and cortical spreading depression. Acta Neurol Scand 113, 1-40.

Lauritzen M. (1994) Pathophysiology of the migraine aura. The spreading depression theory. Brain $117,199-210$.

Lauritzen M., Dreier J.P., Fabricius M., Hartings J.A., Graf R. \& Strong A.J. (2011) Clinical relevance of cortical spreading depression in neurological disorders: migraine, malignant stroke, subarachnoid and intracranial hemorrhage, and traumatic brain injury. $J$ Cereb blood flow Metab 31, 17-35.

Lavenex P. \& Amaral D.G. (2000) Hippocampal-neocortical interaction: A hierarchy of associativity. Hippocampus 10, 420-430.

Leão A.A.P. (1944) Spreading depression of activity in the cerebral cortex. J Neurophysiol 7, 359390.

Leão A.A.P. (1947) Further observations on the spreading depression of activity in the cerebral cortex. J Neurophysiol 10, 409-14.

Lee H.K., Barbarosie M., Kameyama K., Bear M.F. \& Huganir R.L. (2000) Regulation of distinct AMPA receptor phosphorylation sites during bidirectional synaptic plasticity. Nature 405, 9559.

Lee H.K., Kameyama K., Huganir R.L. \& Bear M.F. (1998) NMDA induces long-term synaptic depression and dephosphorylation of the GluR1 subunit of AMPA receptors in hippocampus. Neuron 21, 1151-62.

Lee S.-J.R., Escobedo-Lozoya Y., Szatmari E.M. \& Yasuda R. (2009) Activation of CaMKII in single dendritic spines during long-term potentiation HHS Public Access. Nature 458, 299 304.

Lee V. \& Maguire J. (2014) The impact of tonic GABA A receptor-mediated inhibition on neuronal excitability varies across brain region and cell type. Front Neural Circuits 8, 1-27.

Leonard A.S., Davare M.A., Horne M.C., Garner C.C. \& Hell J.W. (1998) SAP97 Is Associated with the-Amino-3-hydroxy-5-methylisoxazole-4-propionic Acid Receptor GluR1 Subunit. J Biol Chem 273, 19518-19524.

Lerma J. (2003) Roles and rules of kainate receptors in synaptic transmission. Nat Rev Neurosci 4 , 481-495.

Levelt C.N. \& Hübener M. (2012) Critical-period plasticity in the visual cortex. Annu Rev Neurosci 35, 309-30.

Li L.-P., Dustrude E.T., Haulcomb M.M., Abreu A.R., Fitz S.D., Johnson P.L., Thakur G.A., Molosh 
A.I., Lai Y. \& Shekhar A. (2018) PSD95 and nNOS interaction as a novel molecular target to modulate conditioned fear: relevance to PTSD. Trans/ Psychiatry 8, 155.

Liao D., Hessler N.A. \& Malinow R. (1995) Activation of postsynaptically silent synapses during pairing-induced LTP in CA1 region of hippocampal slice. Nature 375, 400-404.

Liao D., Scannevin R.H. \& Huganir R. (2001) Activation of Silent Synapses by Rapid ActivityDependent Synaptic Recruitment of AMPA Receptors. J Neurosci 21, 6008-6017.

Liaw W.-J., Zhu X.-G., Yaster M., Johns R.A., Gauda E.B. \& Tao Y.-X. (2008) Distinct expression of synaptic NR2A and NR2B in the central nervous system and impaired morphine tolerance and physical dependence in mice deficient in postsynaptic density-93 protein. Mol Pain 4.

Lindsberg P.J., Hallenbeck J.M. \& Feuerstein G. (1991) Platelet-activating factor in stroke and brain injury. Ann Neurol 30, 117-29.

Lipp P. \& Reither G. (2011) Protein Kinase C: The Masters of Calcium and Lipid. Cold Spring Harb Perspect Bio/ 3, a004556-a004556.

Lisman J. \& Raghavachari S. (2006) A unified model of the presynaptic and postsynaptic changes during LTP at CA1 synapses. Sci STKE 2006, re11.

Lisman J., Schulman H. \& Cline H. (2002) The molecular basis of CaMKII function in synaptic and behavioural memory. Nat Rev Neurosci 3, 175-90.

Liu F. \& McCullough L.D. (2012) Interactions between age, sex, and hormones in experimental ischemic stroke. Neurochem Int 61, 1255-65.

Liu F., Yuan R., Benashski S.E. \& McCullough L.D. (2009a) Changes in experimental stroke outcome across the life span. $J$ Cereb blood flow Metab 29, 792-802.

Liu L., Zhang R., Liu K., Zhou H., Tang Y., Su J., Yu X., Yang X., Tang M. \& Dong Q. (2009b) Tissue kallikrein alleviates glutamate-induced neurotoxicity by activating ERK1. J Neurosci Res 87, 3576-90.

Lonart G. \& Südhof T.C. (2000) Assembly of SNARE Core Complexes Prior to Neurotransmitter Release Sets the Readily Releasable Pool of Synaptic Vesicles* Gyö rgy Lonart and Downloaded from. J Biol Chem 275, 27703-27707.

Lu W., Shi Y., Jackson A.C., Bjorgan K., During M.J., Sprengel R., Seeburg P.H. \& Nicoll R.A. (2009) Subunit composition of synaptic AMPA receptors revealed by a single-cell genetic approach. Neuron 62, 254-268.

Lüscher C. \& Malenka R.C. (2011) Drug-evoked synaptic plasticity in addiction: from molecular changes to circuit remodeling. Neuron 69, 650-663. 
Macdonald R.L. \& Olsen R.W. (1994) GABA A Receptor Channels. Annu Rev Neurosci 17, 569602.

Maffei A. \& Fontanini A. (2009) Network homeostasis: a matter of coordination Homeostatic regulation of pyramidal cell firing. Curr Opin Neurobiol 19, 168-173.

Makino Y., Johnson R.C., Yu Y., Takamiya K. \& Huganir R.L. (2011) Enhanced synaptic plasticity in mice with phosphomimetic mutation of the GluA1 AMPA receptor. Proc Natl Acad Sci 108, $8450-8455$.

Malinow R. \& Malenka R.C. (2002) AMPA receptor trafficking and synaptic plasticity. Annu Rev Neurosci 25, 103-26.

Malumbres M., Mangues R., Ferrer N., Lu S. \& Pellicer A. (1997) Isolation of high molecular weight DNA for reliable genotyping of transgenic mice. Biotechniques 22, 1114-9.

Mammen A.L., Kameyama K., Roche K.W. \& Huganir R.L. (1997) Phosphorylation of the alphaamino-3-hydroxy-5-methylisoxazole4-propionic acid receptor GluR1 subunit by calcium/calmodulin-dependent kinase II. J Biol Chem 272, 32528-33.

Mané M. \& Müller M. (2012) Temporo-Spectral Imaging of Intrinsic Optical Signals during HypoxiaInduced Spreading Depression-Like Depolarization. PLoS One 7.

Marrannes R., Willems R., De Prins E. \& Wauquier A. (1988) Evidence for a role of the N-methylD-aspartate (NMDA) receptor in cortical spreading depression in the rat. Brain Res 457, 22640.

Marshall W.H. (1959) Spreading cortical depression of Leao. Physiol Rev 39, 239-79.

Martin R.L., Lloyd H.G. \& Cowan A.I. (1994) The early events of oxygen and glucose deprivation: setting the scene for neuronal death? Trends Neurosci 17, 251-7.

Martins-Ferreira H. \& de Castro G.O. (1966) Light-scattering changes accompanying spreading depression in isolated retina. $J$ Neurophysiol 29, 715-26.

Matsui K., Jahr C.E. \& Rubio M.E. (2005) High-concentration rapid transients of glutamate mediate neural-glial communication via ectopic release. J Neurosci 25, 7538-47.

Mayer M., Westbrook G. \& Guthrie P. (1984) Voltage-dependent block by Mg2+ of NMDA responses in spinal cord neurones. Nature 309, 261-3.

Mayevsky A. \& Chance B. (1982) Intracellular oxidation-reduction state measured in situ by a multichannel fiber-optic surface fluorometer. Science 217, 537-40.

McGee A.W., Topinka J.R., Hashimoto K., Petralia R.S., Kakizawa S., Kauer F., Aguilera-Moreno A., Wenthold R.J., Kano M. \& Bredt D.S. (2001) PSD-93 Knock-Out Mice Reveal That 
Neuronal MAGUKs Are Not Required for Development or Function of Parallel Fiber Synapses in Cerebellum. J Neurosci 21, 3085-91.

Mies G. (1997) Blood flow dependent duration of cortical depolarizations in the periphery of focal ischemia of rat brain. Neurosci Lett 221, 165-8.

Migaud M., Charlesworth P., Dempster M., Webster L.C., Watabe A.M., Makhinson M., He Y., Ramsay M.F., Morris R.G., Morrison J.H., O'Dell T.J. \& Grant S.G. (1998) Enhanced longterm potentiation and impaired learning in mice with mutant postsynaptic density-95 protein. Nature 396, 433-9.

Mitchell N.A. \& Fleck M.W. (2007) Targeting AMPA receptor gating processes with allosteric modulators and mutations. Biophys J 92, 2392-2402.

Montgomery J.M., Pavlidis P. \& Madison D. V. (2001) Pair recordings reveal all-silent synaptic connections and the postsynaptic expression of long-term potentiation. Neuron 29, 691-701.

Monyer H., Burnashev N., Laurie D.J., Sakmann B. \& Seeburg P.H. (1994) Developmental and regional expression in the rat brain and functional properties of four NMDA receptors. Neuron 12, 529-40.

Monyer H., Sprengel R., Schoepfer R., Herb A., Higuchi M., Lomeli H., Burnashev N., Sakmann B. \& Seeburg P. (1992) Heteromeric NMDA receptors: molecular and functional distinction of subtypes. Science 256, 1217-21.

Mulkey R.M., Endo S., Shenolikar S. \& Malenka R.C. (1994) Involvement of a calcineurin/inhibitor1 phosphatase cascade in hippocampal long-term depression. Nature 369, 486-8.

Mulkey R.M. \& Malenka R.C. (1992) Mechanisms underlying induction of homosynaptic long-term depression in area CA1 of the hippocampus. Neuron 9, 967-75.

Mulle C., Sailer A., Pérez-Otaño I., Dickinson-Anson H., Castillo P.E., Bureau I., Maron C., Gage F.H., Mann J.R., Bettler B. \& Heinemann S.F. (1998) Altered synaptic physiology and reduced susceptibility to kainate-induced seizures in GluR6-deficient mice. Nature 392, 601-605.

Müller M. (2000) Effects of chloride transport inhibition and chloride substitution on neuron function and on hypoxic spreading-depression-like depolarization in rat hippocampal slices. Neuroscience 97, 33-45.

Müller M. \& Somjen G.G. (1998) Inhibition of major cationic inward currents prevents spreading depression-like hypoxic depolarization in rat hippocampal tissue slices. Brain Res 812, 1-13.

Müller M. \& Somjen G.G. (1999) Intrinsic Optical Signals in Rat Hippocampal Slices During Hypoxia-Induced Spreading Depression-Like Depolarization. J Neurophysiol 82, 1818-1831.

Müller M. \& Somjen G.G. (2000a) Na and K Concentrations, Extra-and Intracellular Voltages, and 
the Effect of TTX in Hypoxic Rat Hippocampal Slices. J Neurophysio/ 83, 735-45.

Müller M. \& Somjen G.G. (2000b) $\mathrm{Na}(+)$ dependence and the role of glutamate receptors and $\mathrm{Na}(+)$ channels in ion fluxes during hypoxia of rat hippocampal slices. J Neurophysiol 84, 1869-80.

Mullis K., Faloona F., Scharf S., Saiki R., Horn G. \& Erlich H. (1986) Specific enzymatic amplification of DNA in vitro: the polymerase chain reaction. Cold Spring Harb Symp Quant Biol 51 Pt 1, 263-73.

Nakagawa T., Futai K., Lashuel H.A., Lo I., Okamoto K., Walz T., Hayashi Y. \& Sheng M. (2004) Quaternary structure, protein dynamics, and synaptic function of SAP97 controlled by L27 domain interactions. Neuron 44, 453-467.

Nasir M.N., Habsah M., Zulkifli M., Zamzuri I., Rammes G., Hasnan J. \& Abdullah J. (2010) Inhibitory effect of asiatic acid on excitatory synaptic transmission in the rat hippocampus. Curr Neurobiol 1.

Nedergaard M. \& Hansen A.J. (1993) Characterization of cortical depolarizations evoked in focal cerebral ischemia. J Cereb blood flow Metab 13, 568-74.

Neves G., Cooke S.F. \& Bliss T.V.P. (2008) Synaptic plasticity, memory and the hippocampus: a neural network approach to causality. Nat Rev Neurosci 9, 65-75.

Nicoll R.A., Malenka R.C. \& Kauer J.A. (1990) Functional comparison of neurotransmitter receptor subtypes in mammalian central nervous system. Physiol Rev 70, 513-65.

Niethammer M., Kim E. \& Sheng M. (1996) Interaction between the C Terminus of NMDA Receptor Subunits and Multiple Members of the PSD-95 Family of Membrane-Associated Guanylate Kinases. J Neurosci 76, 2157-2163.

Nithianantharajah J., Komiyama N.H., Mckechanie A., Johnstone M., Blackwood D.H., St Clair D., Emes R.D., Van De Lagemaat L.N., Saksida L.M., Bussey T.J. \& Grant S.G.N. (2013) Synaptic scaffold evolution generated components of vertebrate cognitive complexity Europe PMC Funders Group. Nat Neurosci 16, 16-24.

Nosyreva E., Szabla K., Autry A.E., Ryazanov A.G., Monteggia L.M. \& Kavalali E.T. (2013) Acute Suppression of Spontaneous Neurotransmission Drives Synaptic Potentiation. J Neurosci 33, $6990-7002$.

Nowak L., Bregestovski P., Ascher P., Herbet A. \& Prochiantz A. (1984) Magnesium gates glutamate-activated channels in mouse central neurones. Nature 307, 462-5.

Nowicky A. V \& Duchen M.R. (1998) Changes in [Ca2+]i and membrane currents during impaired mitochondrial metabolism in dissociated rat hippocampal neurons. J Physiol 507, 131-45. 
Ouanonou A., Zhang Y. \& Zhang L. (1999) Changes in the calcium dependence of glutamate transmission in the hippocampal CA1 region after brief hypoxia-hypoglycemia. $J$ Neurophysiol 82, 1147-55.

Pachernegg S., Strutz-Seebohm N. \& Hollmann M. (2012) GluN3 subunit-containing NMDA receptors: not just one-trick ponies. Trends Neurosci 35, 240-249.

Papadia S. \& Hardingham G.E. (2007) The dichotomy of NMDA receptor signalling. Neuroscientist 13, 572-579.

Parsons M.P. \& Raymond L.A. (2014) Extrasynaptic NMDA receptor involvement in central nervous system disorders. Neuron 82, 279-93.

Peng J., Kim M.J., Cheng D., Duong D.M., Gygi S.P. \& Sheng M. (2004) Semiquantitative proteomic analysis of rat forebrain postsynaptic density fractions by mass spectrometry. J Biol Chem 279, 21003-11.

Pérez-Otaño I. \& Ehlers M.D. (2004) Learning from NMDA Receptor Trafficking: Clues to the Development and Maturation of Glutamatergic Synapses. Neurosignals 13, 175-189.

Petralia R.S. (2012) Distribution of Extrasynaptic NMDA Receptors on Neurons. Sci World J 2012, $1-11$.

Petralia R.S., Esteban J.A., Wang Y.X., Partridge J.G., Zhao H.M., Wenthold R.J. \& Malinow R. (1999) Selective acquisition of AMPA receptors over postnatal development suggests a molecular basis for silent synapses. Nat Neurosci 2, 31-6.

Petralia R.S., Wang Y.-X. \& Wenthold R.J. (1994) Histological and ultrastructural localization of the kainate receptor subunits, KA2 and GluR6/7, in the rat nervous system using selective antipeptide antibodies. J Comp Neurol 349, 85-110.

Petralia R.S., Wang Y.X., Hua F., Yi Z., Zhou A., Ge L., Stephenson F.A. \& Wenthold R.J. (2010) Organization of NMDA receptors at extrasynaptic locations. Neuroscience 167, 68-87.

Petrini E.M., Lu J., Cognet L., Lounis B., Ehlers M.D. \& Choquet D. (2009) Endocytic Trafficking and Recycling Maintain a Pool of Mobile Surface AMPA Receptors Required for Synaptic Potentiation. Neuron 63, 92-105.

Popa-Wagner A., Badan I., Walker L., Groppa S., Patrana N. \& Kessler C. (2007) Accelerated infarct development, cytogenesis and apoptosis following transient cerebral ischemia in aged rats. Acta Neuropathol 113, 277-93.

Pou S., Pou W.S., Bredt D.S., Snyder S.H. \& Rosen G.M. (1992) Generation of superoxide by purified brain nitric oxide synthase. J Biol Chem 267, 24173-6.

Quinlan E.M., Philpot B.D., Huganir R.L. \& Bear M.F. (1999) Rapid, experience-dependent 
expression of synaptic NMDA receptors in visual cortex in vivo. Nat Neurosci 2, 352-357.

Rogawski M.A. (2008) Common pathophysiologic mechanisms in migraine and epilepsy. Arch Neurol 65, 709-14.

Rojas J.I., Zurrú M.C., Romano M., Patrucco L. \& Cristiano E. (2007) Acute ischemic stroke and transient ischemic attack in the very old--risk factor profile and stroke subtype between patients older than 80 years and patients aged less than 80 years. Eur J Neurol 14, 895-9.

Rong R., Yang H., Rong L., Wei X., Li Q., Liu X., Gao H., Xu Y. \& Zhang Q. (2016) Proteomic analysis of PSD-93 knockout mice following the induction of ischemic cerebral injury. Neurotoxicology 53, 1-11.

Rosamond W., Flegal K., Furie K., Go A., Greenlund K., Haase N., Hailpern S.M., Ho M., Howard V., Kissela B., Kissela B., Kittner S., Lloyd-Jones D., McDermott M., Meigs J., Moy C., Nichol G., O'Donnell C., Roger V., Sorlie P., Steinberger J., Thom T., Wilson M., Hong Y. \& American Heart Association Statistics Committee and Stroke Statistics Subcommittee. (2008) Heart disease and stroke statistics--2008 update: a report from the American Heart Association Statistics Committee and Stroke Statistics Subcommittee. Circulation 117, e25146.

Rosenmund C., Stern-Bach Y. \& Stevens C.F. (1998) The tetrameric structure of a glutamate receptor channel. Science 280, 1596-9.

Rosenmund C. \& Stevens C.F. (1996) Definition of the readily releasable pool of vesicles at hippocampal synapses. Neuron 16, 1197-207.

Rubaj A., Zgodziński W. \& Sieklucka-Dziuba M. (2003) The epileptogenic effect of seizures induced by hypoxia: the role of NMDA and AMPA/KA antagonists. Pharmacol Biochem Behav 74, 303-11.

Rumbaugh G., Adams J.P., Kim J.H. \& Huganir R.L. (2006) SynGAP regulates synaptic strength and mitogen-activated protein kinases in cultured neurons. Proc Natl Acad Sci 103, 43444351.

Rumbaugh G., Sia G.-M., Garner C.C. \& Huganir R.L. (2003) Synapse-Associated Protein-97 Isoform-Specific Regulation of Surface AMPA Receptors and Synaptic Function in Cultured Neurons. J Neurosci 23, 4567-4576.

Rungta R.L., Choi H.B., Tyson J.R., Malik A., Dissing-Olesen L., Lin P.J.C., Cain S.M., Cullis P.R., Snutch T.P. \& MacVicar B.A. (2015) The Cellular Mechanisms of Neuronal Swelling Underlying Cytotoxic Edema. Cell 161, 610-621.

Sahay A. \& Hen R. (2008) Hippocampal neurogenesis and depression. Novartis Found Symp 289, 152-160. 
Saiki R.K., Gelfand D.H., Stoffel S., Scharf S.J., Higuchi R., Horn G.T., Mullis K.B. \& Erlich H.A. (1988) Primer-directed enzymatic amplification of DNA with a thermostable DNA polymerase. Science 239, 487-91.

Sambrook J., Fritsch E.F. \& Maniatis T. (1989) Molecular cloning: a laboratory manual. Mol cloning a Lab manual.

Sans N., Petralia R.S., Wang Y.X., Blahos J., Hell J.W. \& Wenthold R.J. (2000) A developmental change in NMDA receptor-associated proteins at hippocampal synapses. $J$ Neurosci 20 , $1260-1271$.

Sattler R., Xiong Z., Lu W.Y., Hafner M., MacDonald J.F. \& Tymianski M. (1999) Specific coupling of NMDA receptor activation to nitric oxide neurotoxicity by PSD-95 protein. Science 284, $1845-8$.

Schlüter O.M., Xu W. \& Malenka R.C. (2006) Alternative N-Terminal Domains of PSD-95 and SAP97 Govern Activity-Dependent Regulation of Synaptic AMPA Receptor Function. Neuron 51, 99-111.

Schmidt-Kastner R. \& Freund T.F. (1991) Selective vulnerability of the hippocampus in brain ischemia. Neuroscience 40, 599-636.

Schmidt-Kastner R. \& Hossmann K.A. (1988) Distribution of ischemic neuronal damage in the dorsal hippocampus of rat. Acta Neuropathol 76, 411-21.

Schnell E., Sizemore M., Karimzadegan S., Chen L., Bredt D.S. \& Nicoll R.A. (2002) Direct interactions between PSD-95 and stargazin control synaptic AMPA receptor number. Proc Natl Acad Sci 99, 13902-13907.

Schorge S. \& Colquhoun D. (2003) Studies of NMDA Receptor Function and Stoichiometry with Truncated and Tandem Subunits. J Neurosci 23, 1151-1158.

Schulman H. (1993) The multifunctional Ca2+/calmodulin-dependent protein kinases. Curr Opin Cell Biol 5, 247-53.

Schuman E.M. \& Madison D. V. (1994) Nitric oxide and synaptic function. Annu Rev Neurosci 17, 153-83.

Semyanov A., Walker M.C. \& Kullmann D.M. (2003) GABA uptake regulates cortical excitability via cell type-specific tonic inhibition. Nat Neurosci 6, 484-490.

Sernagor E., Chabrol F., Bony G. \& Cancedda L. (2010) GABAergic control of neurite outgrowth and remodeling during development and adult neurogenesis: general rules and differences in diverse systems. Front Cell Neurosci 4, 11.

Shapiro A.L., Viñuela E. \& V. Maizel J. (1967) Molecular weight estimation of polypeptide chains by 
electrophoresis in SDS-polyacrylamide gels. Biochem Biophys Res Commun 28, 815-820.

Shen K. \& Meyer T. (1999) Dynamic control of CaMKII translocation and localization in hippocampal neurons by NMDA receptor stimulation. Science 284, 162-6.

Sheng M., Cummings J., Roldan L.A., Jan Y.N. \& Jan L.Y. (1994) Changing subunit composition of heteromeric NMDA receptors during development of rat cortex. Nature 368, 144-7.

Sheng M. \& Kim E. (1996) Ion channel associated proteins. Curr Opin Neurobio/ 6, 602-608.

Shepherd J.D. \& Huganir R.L. (2007) The cell biology of synaptic plasticity: AMPA receptor trafficking. Annu Rev Cell Dev Biol 23, 613-43.

Shi J.-M., Pei J., Liu E.-Q. \& Zhang L. (2017) Bis(sulfosuccinimidyl) suberate (BS3) crosslinking analysis of the behavior of amyloid- $\beta$ peptide in solution and in phospholipid membranes. PLoS One 12, e0173871.

Shi Z.-Q., Sunico C.R., McKercher S.R., Cui J., Feng G.-S., Nakamura T. \& Lipton S.A. (2013) Snitrosylated SHP-2 contributes to NMDA receptor-mediated excitotoxicity in acute ischemic stroke. Proc Natl Acad Sci U S A 110, 3137-42.

Shukla A., Beroun A., Panopoulou M., Neumann P.A., Grant S.G., Olive M.F., Dong Y. \& Schlüter O.M. (2017) Calcium-permeable AMPA receptors and silent synapses in cocaine-conditioned place preference. EMBO J 36, 458-474.

Silva A.J., Stevens C.F., Tonegawa S. \& Wang Y. (1992) Deficient hippocampal long-term potentiation in alpha-calcium-calmodulin kinase II mutant mice. Science 257, 201-6.

Soderling T.R. (2000) CaM-kinases: modulators of synaptic plasticity. Curr Opin Neurobiol 10, 375-80.

Somjen G.G. (2001) Mechanisms of Spreading Depression and Hypoxic Spreading DepressionLike Depolarization. Physiol Rev 81, 1065-96.

Somjen G.G. \& Aitken P.G. (1984) The ionic and metabolic responses associated with neuronal depression of Leão's type in cerebral cortex and in hippocampal formation. An Acad Bras Cienc 56, 495-504.

Somjen G.G., Aitken P.G., Czéh G., Jing J. \& Young J.N. (1993) Cellular physiology of hypoxia of the mammalian central nervous system. Res Publ Assoc Res Nerv Ment Dis 71, 51-65.

Squire L.R. (1992) Memory and the hippocampus: a synthesis from findings with rats, monkeys, and humans. Psychol Rev 99, 195-231.

Stein V., House D.R.C., Bredt D.S. \& Nicoll R.A. (2003) Postsynaptic Density-95 Mimics and Occludes Hippocampal Long-Term Potentiation and Enhances Long-Term Depression. $J$ 
Neurosci 23, 5503-5506.

Stricker N.L., Christopherson K.S., Yi B.A., Schatz P.J., Raab R.W., Dawes G., Bassett D.E., Bredt D.S. \& Li M. (1997) PDZ domain of neuronal nitric oxide synthase recognizes novel Cterminal peptide sequences. Nat Biotechnol 15, 336-342.

Strong A.J., Fabricius M., Boutelle M.G., Hibbins S.J., Hopwood S.E., Jones R., Parkin M.C. \& Lauritzen M. (2002) Spreading and synchronous depressions of cortical activity in acutely injured human brain. Stroke 33, 2738-43.

Südhof T.C. (2002) Synaptotagmins: why so many? J Biol Chem 277, 7629-32.

Sun H.-S., Doucette T.A., Liu Y., Fang Y., Teves L., Aarts M., Ryan C.L., Bernard P.B., Lau A., Forder J.P., Salter M.W., Wang Y.T., Tasker R.A. \& Tymianski M. (2008) Effectiveness of PSD95 Inhibitors in Permanent and Transient Focal Ischemia in the Rat. Stroke 39, 25442553.

Sun L., Margolis F.L., Shipley M.T. \& Lidow M.S. (1998) Identification of a long variant of mRNA encoding the NR3 subunit of the NMDA receptor: its regional distribution and developmental expression in the rat brain. FEBS Lett 441, 392-396.

Sun M.-K., Xu H. \& Alkon D.L. (2002) Pharmacological protection of synaptic function, spatial learning, and memory from transient hypoxia in rats. $J$ Pharmacol Exp Ther 300, 408-16.

Sun Q. \& Turrigiano G.G. (2011) PSD-95 and PSD-93 play critical but distinct roles in synaptic scaling up and down. $J$ Neurosci 31, 6800-8.

Sun Y., Ikrar T., Davis M.F., Gong N., Zheng X., Luo Z.D., Lai C., Mei L., Holmes T.C., Gandhi S.P. \& Xu X. (2016) Neuregulin-1/ErbB4 Signaling Regulates Visual Cortical Plasticity. Neuron 92, $160-173$.

Szatkowski M. \& Attwell D. (1994) Triggering and execution of neuronal death in brain ischaemia: two phases of glutamate release by different mechanisms. Trends Neurosci 17, 359-65.

Szepesi Z., Hosy E., Ruszczycki B., Bijata M. \& Pyskaty M. (2014) Synaptically Released Matrix Metalloproteinase Activity in Control of Structural Plasticity and the Cell Surface Distribution of GluA1-AMPA Receptors. PLoS One 9, 98274.

Taha S., Hanover J.L., Silva A.J. \& Stryker M.P. (2002) Autophosphorylation of alphaCaMKII is required for ocular dominance plasticity. Neuron 36, 483-91.

Takagi N., Logan R., Teves L., Wallace M.C. \& Gurd J.W. (2000) Altered interaction between PSD95 and the NMDA receptor following transient global ischemia. J Neurochem 74, 169-78.

Takeuchi M., Hata Y., Hirao K., Toyoda A., Irie M. \& Takai Y. (1997) SAPAPs - A family of PSD95/SAP90-associated proteins localized at postsynaptic density. J Biol Chem 272, 11943- 
11951.

Tao Y.-X., Rumbaugh G., Wang G.-D., Petralia R.S., Zhao C., Kauer F.W., Tao F., Zhuo M., Wenthold R.J., Raja S.N., Huganir R.L., Bredt D.S. \& Johns R.A. (2003) Impaired NMDA Receptor-Mediated Postsynaptic Function and Blunted NMDA Receptor-Dependent Persistent Pain in Mice Lacking Postsynaptic Density-93 Protein. J Neurosci 23, 6703-3712.

Tillmann S., Pereira S., Liebenberg N., Christensen A.K. \& Wegener G. (2017) ZL006, a small molecule inhibitor of PSD-95/ nNOS interaction, does not induce antidepressant-like effects in two genetically predisposed rat models of depression and control animals. PLoS One 12, e0182698.

Toro C. \& Deakin J.F.W. (2005) NMDA receptor subunit NRI and postsynaptic protein PSD-95 in hippocampus and orbitofrontal cortex in schizophrenia and mood disorder. Schizophr Res $\mathbf{8 0}$, 323-30.

Tovar K.R. \& Westbrook G.L. (1999) The incorporation of NMDA receptors with a distinct subunit composition at nascent hippocampal synapses in vitro. J Neurosci 19, 4180-8.

Towbin H., Staehelin T. \& Gordon J. (1992) Electrophoretic transfer of proteins from polyacrylamide gels to nitrocellulose sheets: procedure and some applications. 1979. Biotechnology 24, 145-9.

Traynelis S.F., Wollmuth L.P., Mcbain C.J., Menniti F.S., Vance K.M., Ogden K.K., Hansen K.B., Yuan H., Myers S.J. \& Dingledine R. (2010) Glutamate Receptor Ion Channels: Structure, Regulation, and Function. Pharmacol Rev 62, 405-96.

Turrigiano G.G., Leslie K.R., Desai N.S., Rutherford L.C. \& Nelson S.B. (1998) Activity-dependent scaling of quantal amplitude in neocortical neurons. Nature 391, 892-6.

Ulbrich M.H. \& Isacoff E.Y. (2007) Subunit counting in membrane-bound proteins. Nat Methods 4, 319-321.

Vicini S., Feng Wang J., Hong J.L., Jian Zhu W., Hua Wang Y., Hong Luo J., Wolfe B.B., Grayson D.R. \& Hong Li J. (1998) Functional and Pharmacological Differences Between Recombinant N-Methyl-D-Aspartate Receptors. J Neurophysiol 79, 555-566.

Vitureira N., Letellier M. \& Goda Y. (2012) Homeostatic synaptic plasticity: from single synapses to neural circuits. Curr Opin Neurobiol 22, 516-521.

Volbracht C., Chua B.T., Ng C.P., Bahr B.A., Hong W. \& Li P. (2005) The critical role of calpain versus caspase activation in excitotoxic injury induced by nitric oxide. J Neurochem 93,1280 1292.

Vyskoeil F., Kritz N. \& Bures J. (1972) Potassium-selective microelectrodes used for measuring the 
extracellular brain potassium during spreading depression and anoxic depolarization in rats. Brain Res 39, 255-9.

Waites C.L., Craig A.M. \& Garner C.C. (2005) Mechanisms of vertebrate synaptogenesis. Annu Rev Neurosci 28, 251-74.

Wang C.-Y., Chang K., Petralia R.S., Wang Y.-X., Seabold G.K. \& Wenthold R.J. (2006) A Novel Family of Adhesion-Like Molecules That Interacts with the NMDA Receptor. J Neurosci 26, 2174-2183.

Wang D.D. \& Kriegstein A.R. (2011) Blocking Early GABA Depolarization with Bumetanide Results in Permanent Alterations in Cortical Circuits and Sensorimotor Gating Deficits. Cereb Cortex March 21, 574-587.

Wegener G. \& Volke V. (2010) Nitric Oxide Synthase Inhibitors as Antidepressants. Pharmaceuticals 3, 273-299.

Wenthold R.J., Petralia R.S. \& Niedzielski A.S. (1996) Evidence for Multiple AMPA Receptor Complexes in Hippocampal CA1/CA2 Neurons. J Neurosci 76, 1982-1989.

Winkler D., Daher F., Wüstefeld L., Hammerschmidt K., Poggi G., Seelbach A., Krueger-Burg D., Vafadari B., Ronnenberg A., Liu Y., Kaczmarek L., Schlüter O.M., Ehrenreich H. \& Dere E. (2018) Hypersocial behavior and biological redundancy in mice with reduced expression of PSD95 or PSD93. Behav Brain Res 352, 35-45.

Wisden W. \& Seeburg P.H. (1993) Mammalian ionotropic glutamate receptors. Curr Opin Neurobiol 3, 291-8.

Woods D.F. \& Bryant P.J. (1991) The discs-large tumor suppressor gene of Drosophila encodes a guanylate kinase homolog localized at septate junctions. Cell 66, 451-64.

Wu C. \& Sun D. (2015) GABA receptors in brain development, function, and injury. Metab Brain Dis 30, 367-79.

Wu D., Bacaj T., Morishita W., Goswami D., Arendt K.L., Xu W., Chen L., Malenka R.C. \& Südhof T.C. (2017) Postsynaptic synaptotagmins mediate AMPA receptor exocytosis during LTP. Nature 544, 316-321.

Wu G., Malinow R. \& Cline H.T. (1996) Maturation of a central glutamatergic synapse. Science 274, 972-6.

Wu Q.J. \& Tymianski M. (2018) Targeting NMDA receptors in stroke: new hope in neuroprotection. Mol Brain 11, 15.

Xu J., Kurup P., Zhang Y., Goebel-Goody S.M., Wu P.H., Hawasli A.H., Baum M.L., Bibb J.A. \& Lombroso P.J. (2009) Extrasynaptic NMDA receptors couple preferentially to excitotoxicity via 
calpain-mediated cleavage of STEP. J Neurosci $\mathbf{2 9}$, 9330-43.

Xu W., Schlüter O.M., Steiner P., Czervionke B.L., Sabatini B. \& Malenka R.C. (2008) Molecular Dissociation of the Role of PSD-95 in Regulating Synaptic Strength and LTD. Neuron 57, 248-262.

Xu Y., Zhang B., Hua Z., Johns R.A., Bredt D.S. \& Tao Y.-X. (2004) Targeted disruption of PSD-93 gene reduces platelet-activating factor-induced neurotoxicity in cultured cortical neurons. Exp Neurol 189, 16-24.

Yanagisawa T., Tsumoto T. \& Kimura F. (2004) Transiently higher release probability during critical period at thalamocortical synapses in the mouse barrel cortex: relevance to differential shortterm plasticity of AMPA and NMDA EPSCs and possible involvement of silent synapses. Eur J Neurosci 20, 3006-18.

Yao W.-D., Gainetdinov R.R., Arbuckle M.I., Sotnikova T.D., Cyr M., Beaulieu J.-M., Torres G.E., Grant S.G.. \& Caron M.G. (2004) Identification of PSD-95 as a Regulator of DopamineMediated Synaptic and Behavioral Plasticity. Neuron 41, 625-638.

Yu H.-M., Xu J., Li C., Zhou C., Zhang F., Han D. \& Zhang G.-Y. (2008) Coupling between neuronal nitric oxide synthase and glutamate receptor 6-mediated c-Jun $\mathrm{N}$-terminal kinase signaling pathway via S-nitrosylation contributes to ischemia neuronal death. Neuroscience $155,1120-32$.

Yue T.L. \& Feuerstein G.Z. (1994) Platelet-activating factor: a putative neuromodulator and mediator in the pathophysiology of brain injury. Crit Rev Neurobio/ 8, 11-24.

Zhang M., Li Q., Chen L., Li J., Zhang X., Chen X., Zhang Q., Shao Y. \& Xu Y. (2014) PSD-93 deletion inhibits Fyn-mediated phosphorylation of NR2B and protects against focal cerebral ischemia. Neurobiol Dis 68, 104-111.

Zhang M., Xu J.T., Zhu X., Wang Z., Zhao X., Hua Z., Tao Y.X. \& Xu Y. (2010) Postsynaptic density-93 deficiency protects cultured cortical neurons from $\mathrm{N}$-methyl-d-aspartate receptortriggered neurotoxicity. Neuroscience 166, 1083-1090.

Zhang Q., Cheng H., Rong R., Yang H., Ji Q., Li Q., Rong L., Hu G. \& Xu Y. (2015) The Effect of PSD-93 Deficiency on the Expression of Early Inflammatory Cytokines Induced by Ischemic Brain Injury. Cell Biochem Biophys 73, 695-700.

Zhou L., Li F., Xu H.B., Luo C.X., Wu H.Y., Zhu M.M., Lu W., Ji X., Zhou Q.G. \& Zhu D.Y. (2010) Treatment of cerebral ischemia by disrupting ischemia-induced interaction of nNOS with PSD95. Nat Med 16, 1439-1444.

Zhou W., Zhang L., Guoxiang X., Mojsilovic-Petrovic J., Takamaya K., Sattler R., Huganir R. \& Kalb R. (2008) GluR1 controls dendrite growth through its binding partner, SAP97. J Neurosci 
28, 10220-33.

Zhu J.J., Esteban J.A., Hayashi Y. \& Malinow R. (2000) Postnatal synaptic potentiation: Delivery of GluR4-containing AMPA receptors by spontaneous activity. Nat Neurosci 3, 1098-1106.

Ziff E.B. (1997) Enlightening the postsynaptic density. Neuron 19, 1163-74. 


\section{APPENDIX}

Table 15: fEPSP slope/FV amplitude ratios of $\mathrm{I}-\mathrm{O}$ curves.

\begin{tabular}{|c|c|c|c|c|c|c|c|c|c|c|c|c|}
\hline \multirow{3}{*}{$\begin{array}{c}\text { Stimulus } \\
\text { intensity }[\mu \mathrm{A}] \\
10\end{array}$} & \multicolumn{12}{|c|}{ fEPSP slope/FV amplitude $\left[\mathrm{ms}^{-1}\right]$; mean \pm SEM } \\
\hline & \multicolumn{3}{|c|}{ WT } & \multicolumn{3}{|c|}{ PSD-95 KO } & \multicolumn{3}{|c|}{ PSD-93 KO } & \multicolumn{3}{|c|}{ 93-95 DKO } \\
\hline & 0.05 & \pm & 0.03 & 0.03 & \pm & 0.02 & 0.06 & \pm & 0.03 & 0.03 & \pm & 0.03 \\
\hline 20 & 0.28 & \pm & 0.06 & 0.17 & \pm & 0.06 & 0.18 & \pm & 0.05 & 0.07 & \pm & 0.03 \\
\hline 30 & 0.47 & \pm & 0.10 & 0.35 & \pm & 0.10 & 0.39 & \pm & 0.08 & 0.14 & \pm & 0.05 \\
\hline 40 & 0.90 & \pm & 0.18 & 0.26 & \pm & 0.07 & 0.66 & \pm & 0.12 & 0.25 & \pm & 0.13 \\
\hline 50 & 1.09 & \pm & 0.22 & 0.33 & \pm & 0.06 & 1.20 & \pm & 0.33 & 0.15 & \pm & 0.03 \\
\hline 60 & 1.31 & \pm & 0.29 & 0.53 & \pm & 0.17 & 1.31 & \pm & 0.29 & 0.23 & \pm & 0.07 \\
\hline 70 & 1.42 & \pm & 0.23 & 0.56 & \pm & 0.17 & 1.53 & \pm & 0.36 & 0.19 & \pm & 0.02 \\
\hline 80 & 1.60 & \pm & 0.30 & 0.59 & \pm & 0.21 & 1.85 & \pm & 0.43 & 0.17 & \pm & 0.04 \\
\hline 90 & 1.63 & \pm & 0.31 & 0.60 & \pm & 0.14 & 1.97 & \pm & 0.49 & 0.20 & \pm & 0.05 \\
\hline 100 & 1.86 & \pm & 0.35 & 0.67 & \pm & 0.22 & 2.27 & \pm & 0.52 & 0.35 & \pm & 0.08 \\
\hline 110 & 2.00 & \pm & 0.38 & 0.68 & \pm & 0.18 & 2.51 & \pm & 0.61 & 0.31 & \pm & 0.09 \\
\hline 120 & 1.93 & \pm & 0.35 & 0.73 & \pm & 0.20 & 2.50 & \pm & 0.55 & 0.35 & \pm & 0.07 \\
\hline 130 & 2.03 & \pm & 0.37 & 0.78 & \pm & 0.18 & 2.66 & \pm & 0.60 & 0.34 & \pm & 0.09 \\
\hline 140 & 2.09 & \pm & 0.38 & 0.79 & \pm & 0.25 & 2.64 & \pm & 0.61 & 0.33 & \pm & 0.07 \\
\hline 150 & 2.06 & \pm & 0.36 & 0.73 & \pm & 0.23 & 2.74 & \pm & 0.59 & 0.36 & \pm & 0.07 \\
\hline
\end{tabular}

Table 16: EPSP2/EPSP1 amplitude ratios of paired-pulse facilitation.

\begin{tabular}{|c|c|c|c|c|c|c|c|c|c|c|c|c|}
\hline \multirow{3}{*}{$\begin{array}{c}\text { Interstimulus } \\
\text { interval [ms] } \\
25\end{array}$} & \multicolumn{12}{|c|}{ Paired-pulse ratio; mean \pm SEM } \\
\hline & \multicolumn{3}{|c|}{ WT } & \multicolumn{3}{|c|}{ PSD-95 KO } & \multicolumn{3}{|c|}{ PSD-93 KO } & \multicolumn{3}{|c|}{ 93-95 DKO } \\
\hline & 3.62 & \pm & 0.54 & 2.15 & \pm & 0.11 & 3.40 & \pm & 0.40 & 2.16 & \pm & 0.16 \\
\hline 50 & 2.91 & \pm & 0.41 & 1.80 & \pm & 0.11 & 2.90 & \pm & 0.39 & 1.73 & \pm & 0.11 \\
\hline 75 & 2.24 & \pm & 0.19 & 1.64 & \pm & 0.10 & 2.42 & \pm & 0.30 & 1.58 & \pm & 0.08 \\
\hline 100 & 2.16 & \pm & 0.26 & 1.54 & \pm & 0.09 & 2.22 & \pm & 0.20 & 1.61 & \pm & 0.11 \\
\hline 125 & 1.90 & \pm & 0.21 & 1.48 & \pm & 0.05 & 2.04 & \pm & 0.18 & 1.60 & \pm & 0.08 \\
\hline 150 & 1.81 & \pm & 0.16 & 1.40 & \pm & 0.06 & 2.08 & \pm & 0.24 & 1.34 & \pm & 0.15 \\
\hline 175 & 1.52 & \pm & 0.07 & 1.35 & \pm & 0.05 & 2.04 & \pm & 0.29 & 1.36 & \pm & 0.09 \\
\hline 200 & 1.63 & \pm & 0.12 & 1.33 & \pm & 0.07 & 1.92 & \pm & 0.22 & 1.39 & \pm & 0.10 \\
\hline
\end{tabular}


A

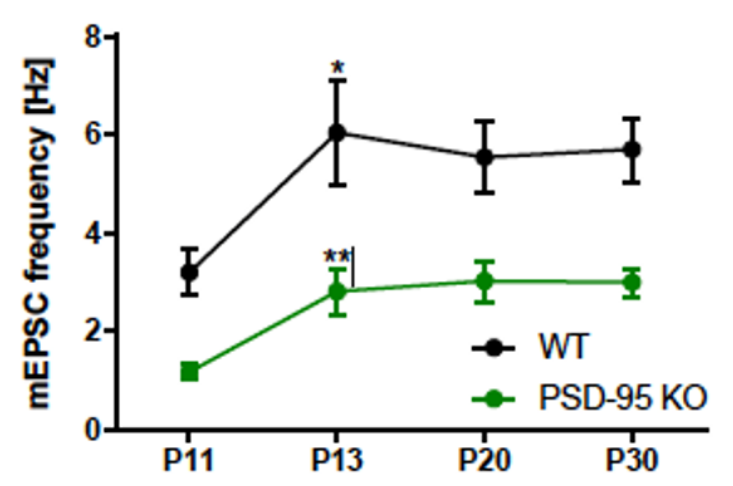

B

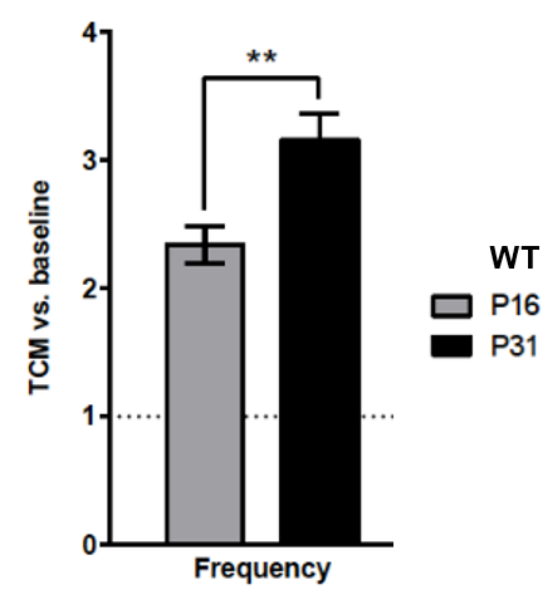

Figure 21: Developmental profile of AMPAR-mEPSC frequency and effect of TCM. Data was provided from (A) Man Ho Wong (PhD thesis, 2017) and (B) Yue Yang (Pittsburgh, unpublished). (A) AMPAR-mEPSC recording of pyramidal neurons in layer II/III of V1 (holding potential of $-60 \mathrm{mV} ; 500 \mathrm{nM}$ TTX to prevent spontaneous action potentials by blocking of voltagegated sodium channels). Graphs show mEPSC frequency during development in WT and PSD-95 KO mice. Welch's t-test: WT P11 vs. P13: $p=0.028$; PSD-95 KO P11 vs. P13: $p=0.007$. ${ }^{*} p<$ $0.05 ;{ }^{* *} p<0.01$. Data were given as means \pm SEM. (B) Experimental set-up according to (A) with additional TCM-treatment of $0.5 \mathrm{mM}$. Bar graphs display the effect of the desensitization blocker TCM on AMPAR-mEPSC frequency in WT mice. TCM provoked a greater increase in mEPSC frequency at P31 $(n=27 ; 3.15$-fold change) than at P16 $(n=27 ; 2.34$-fold change). Welch's t-test: $\mathrm{P} 16$ vs. $\mathrm{P} 31, \mathrm{p}=0.0025 .{ }^{* *} \mathrm{p}<0.01$. Data were given as means $\pm \mathrm{SEM}$. 
Table 17: Statistical analysis.

\begin{tabular}{lllll}
\hline & Statistical test & Comparison & F value & P value \\
Figure 4A: I-O curve & & & $F_{14,700=33.078}$ & $p<0.001$ \\
\cline { 3 - 5 } & 2-way ANOVA & Stimulus & $F_{3,50=7.422}$ & $p<0.001$ \\
& & Genotype & $F_{42,700=5.827}$ & $p<0.001$ \\
\hline \multirow{2}{*}{ Post-hoc LSD } & Interaction & & $p=0.012$ \\
& & PSD-95 KO vs. WT & $p=0.280$ \\
\hline & DKO vs. WT & $p=0.004$ \\
\hline & PSD-95 KO vs. PSD-93 KO & $p=0.001$ \\
\hline & PSD-95 KO vs. DKO & $p=0.458$ \\
\hline
\end{tabular}

Figure 4B: Paired-pulse ratio

$\begin{array}{llll}\text { 2-way ANOVA } & \text { Interstimulus interval } & \mathrm{F}_{7,371}=33.046 & \mathrm{p}<0.001 \\ & \text { Genotype } & \mathrm{F}_{3,53}=4.789 & \mathrm{p}=0.005 \\ \text { Post-hoc LSD } & \text { Interaction } & \mathrm{F}_{21,371}=2.107 & \mathrm{p}=0.003 \\ & \text { PSD-95 KO vs. WT } & & \mathrm{p}=0.015 \\ & \text { PSD-93 KO vs. WT } & & \mathrm{p}=0.535 \\ & \text { DKO vs. WT } & \mathrm{p}=0.036 \\ & \text { PSD-95 KO vs. PSD-93 KO } & \mathrm{p}=0.003 \\ & \text { PSD-95 KO vs. DKO } & \mathrm{p}=0.935 \\ & \text { PSD-93 KO vs. DKO } & \mathrm{p}=0.009\end{array}$

Figure 7A: Time to HSD onset

$\begin{array}{llll}\text { 1-way ANOVA } & \text { Genotype } & F_{3,184}=5.787 & \mathrm{p}=0.001 \\ \text { Post-hoc LSD } & \text { PSD-95 KO vs. WT } & \mathrm{p}=0.002 \\ & \text { PSD-93 KO vs. WT } & \mathrm{p}=0.241 \\ & \text { DKO vs. WT } & \mathrm{p}=0.504 \\ & \text { PSD-95 KO vs. PSD-93 KO } & \mathrm{p}=0.041 \\ & \text { PSD-95 KO vs. DKO } & \mathrm{p}<0.001 \\ & \text { PSD-93 KO vs. DKO } & \mathrm{p}=0.062\end{array}$

Figure 7B: HSD amplitude

1-way ANOVA Genotype $\quad F_{3,184}=0.327 \quad p=0.806$

Figure 7C: HSD duration

$\begin{array}{lllll}1 \text { min } & 1 \text {-way ANOVA } & \text { Genotype } & F_{3,64}=0.953 & p=0.420 \\ 2 \text { min } & 1 \text {-way ANOVA } & \text { Genotype } & F_{3,59}=0.670 & p=0.574 \\ 4 \text { min } & 1 \text { 1-way ANOVA } & \text { Genotype } & F_{3,53}=0.803 & p=0.498\end{array}$




\begin{tabular}{|c|c|c|c|c|}
\hline & Statistical test & Comparison & F value & $P$ value \\
\hline \multicolumn{5}{|c|}{ Figure 8D: Reflectance increase } \\
\hline $1 \mathrm{~min}$ & 1-way ANOVA & Genotype & $\mathrm{F}_{3,55}=7.617$ & $p<0.001$ \\
\hline & Post-hoc LSD & PSD-95 KO vs. WT & & $p<0.001$ \\
\hline & & PSD-93 KO vs. WT & & $p=0.691$ \\
\hline & & DKO vs. WT & & $p=0.080$ \\
\hline & & PSD-95 KO vs. PSD-93 KO & & $p<0.001$ \\
\hline & & PSD-95 KO vs. DKO & & $p=0.016$ \\
\hline & & PSD-93 KO vs. DKO & & $p=0.157$ \\
\hline \multirow[t]{7}{*}{$2 \min$} & 1-way ANOVA & Genotype & $F_{3,58}=3.720$ & $p=0.016$ \\
\hline & Post-hoc LSD & PSD-95 KO vs. WT & & $p=0.020$ \\
\hline & & PSD-93 KO vs. WT & & $p=0.566$ \\
\hline & & DKO vs. WT & & $p=0.874$ \\
\hline & & PSD-95 KO vs. PSD-93 KO & & $p=0.003$ \\
\hline & & PSD-95 KO vs. DKO & & $p=0.015$ \\
\hline & & PSD-93 KO vs. DKO & & $p=0.689$ \\
\hline $4 \mathrm{~min}$ & 1-way ANOVA & Genotype & $\mathrm{F}_{3,48}=1.755$ & $p=0.168$ \\
\hline
\end{tabular}

Figure 9A: HSD-invaded area

1-way ANOVA Genotype $\quad F_{3,170}=0.864 \quad p=0.461$

Figure 9B: HSD propagation velocity

\begin{tabular}{|c|c|c|}
\hline 1-way ANOVA & $F_{3,175}=2.508$ & $p=0.061$ \\
\hline \multirow[t]{6}{*}{ Post-hoc LSD } & PSD-95 KO vs. WT & $p=0.036$ \\
\hline & PSD-93 KO vs. WT & $p=0.934$ \\
\hline & DKO vs. WT & $p=0.798$ \\
\hline & PSD-95 KO vs. PSD-93 KO & $p=0.039$ \\
\hline & PSD-95 KO vs. DKO & $p=0.017$ \\
\hline & PSD-93 KO vs. DKO & $p=0.730$ \\
\hline
\end{tabular}

Figure 10: EPSP amplitude: baseline

\begin{tabular}{|c|c|c|c|}
\hline $\begin{array}{l}\text { 1-way ANOVA } \\
\text { Post-hoc LSD }\end{array}$ & Genotype & $\mathrm{F}_{3,184}=53.246$ & $\mathrm{p}<0.001$ \\
\hline \multirow[t]{6}{*}{ Post-hoc LSD } & PSD-95 KO vs. WT & & $p<0.001$ \\
\hline & PSD-93 KO vs. WT & & $p=0.090$ \\
\hline & DKO vs. WT & & $p<0.001$ \\
\hline & PSD-95 KO vs. PSD-93 KO & & $p<0.001$ \\
\hline & PSD-95 KO vs. DKO & & $p=0.150$ \\
\hline & PSD-93 KO vs. DKO & & $p<0.001$ \\
\hline
\end{tabular}




\section{$\begin{array}{llll}\text { Statistical test } & \text { Comparison } & \text { F value } & \text { P value }\end{array}$}

Figure 12: EPSP amplitude: posthypoxic recovery

\begin{tabular}{|c|c|c|c|c|}
\hline \multirow[t]{7}{*}{ A. $1 \mathrm{~min}$} & 2-way ANOVA & Genotype & $F_{3,63}=4.204$ & $p=0.009$ \\
\hline & Post-hoc LSD & PSD-95 KO vs. WT & & $p=0.004$ \\
\hline & & PSD-93 KO vs. WT & & $p=0.132$ \\
\hline & & DKO vs. WT & & $p=0.003$ \\
\hline & & PSD-95 KO vs. PSD-93 KO & & $p=0.169$ \\
\hline & & PSD-95 KO vs. DKO & & $p=0.798$ \\
\hline & & PSD-93 KO vs. DKO & & $p=0.118$ \\
\hline \multirow[t]{7}{*}{ B. $2 \min$} & 2-way ANOVA & Genotype & $F_{3,61}=8.458$ & $p<0.001$ \\
\hline & Post-hoc LSD & PSD-95 KO vs. WT & & $p=0.003$ \\
\hline & & PSD-93 KO vs. WT & & $p=0.642$ \\
\hline & & DKO vs. WT & & $p<0.001$ \\
\hline & & PSD-95 KO vs. PSD-93 KO & & $p=0.008$ \\
\hline & & PSD-95 KO vs. DKO & & $p=0.220$ \\
\hline & & PSD-93 KO vs. DKO & & $\mathrm{p}<0.001$ \\
\hline \multirow[t]{7}{*}{ Figure 12C. $4 \mathrm{~min}$} & 2-way ANOVA & Genotype & $F_{3,52}=18.391$ & $p<0.001$ \\
\hline & Post-hoc LSD & PSD-95 KO vs. WT & & $\mathrm{p}<0.001$ \\
\hline & & PSD-93 KO vs. WT & & $p=0.744$ \\
\hline & & DKO vs. WT & & $\mathrm{p}<0.001$ \\
\hline & & PSD-95 KO vs. PSD-93 KO & & $p<0.001$ \\
\hline & & PSD-95 KO vs. DKO & & $p=0.463$ \\
\hline & & PSD-93 KO vs. DKO & & $p<0.001$ \\
\hline
\end{tabular}

Figure 13: Total protein level

Figure 13B: WT

\begin{tabular}{|c|c|c|c|c|}
\hline $\mathrm{GABA}_{\alpha 1}$ & 1-way ANOVA & Age & $F_{a g}(2,10)=35.650$ & $p_{a g}<0.001$ \\
\hline & Post-hoc LSD & P13-14 vs. P20-21 & & $p=0.011$ \\
\hline & & P13-14 vs. P28-32 & & $p<0.001$ \\
\hline & & P20-21 vs. P28-32 & & $p<0.001$ \\
\hline \multirow[t]{4}{*}{ GluN2A } & 1-way ANOVA & Age & $F_{\mathrm{ag}(2,10)}=6.348$ & $p_{a g}=0.017$ \\
\hline & Post-hoc LSD & P13-14 vs. P20-21 & & $p=0.078$ \\
\hline & & P13-14 vs. P28-32 & & $p=0.005$ \\
\hline & & P20-21 vs. P28-32 & & $p=0.285$ \\
\hline \multirow[t]{4}{*}{ GluN2B } & 1-way ANOVA & Age & $F_{a g(2,10)}=6.651$ & $p_{a g}=0.015$ \\
\hline & Post-hoc LSD & P13-14 vs. P20-21 & & $p=0.500$ \\
\hline & & P13-14 vs. P28-32 & & $p=0.021$ \\
\hline & & P20-21 vs. P28-32 & & $p=0.009$ \\
\hline GluA1 & 1-way ANOVA & Age & $\mathrm{F}_{\mathrm{ag}(2,10)}=1.697$ & $p_{a g}=0.232$ \\
\hline GluA2 & 1-way ANOVA & Age & $\mathrm{F}_{\mathrm{ag}(2,10)}=2.289$ & $p_{a g}=0.152$ \\
\hline
\end{tabular}




\begin{tabular}{clllll}
\hline \multicolumn{2}{c}{ Statistical test } & Comparison & F value & P value \\
\hline Figure 13C: PSD-95 KO & & & \\
\hline GABA $\alpha 1$ & 1-way ANOVA & Age & $F_{a g(2,10)}=15.623$ & $p_{a g}=0.001$ \\
& Post-hoc LSD & P13-14 vs. P20-21 & & $p=0.553$ \\
\hline & & P13-14 vs. P28-32 & $p=0.001$ \\
\hline GluN2A & 1-way ANOVA & Age & & $p=0.001$ \\
\hline GluN2B & 1-way ANOVA & Age & $F_{a g(2,10)}=2.165$ & $p_{a g}=0.165$ \\
GluA1 & 1-way ANOVA & Age & $F_{a g(2,10)}=0.146$ & $p_{a g}=0.866$ \\
GluA2 & 1-way ANOVA & Age & $F_{a g(2,10)}=0.724$ & $p_{a g}=0.508$ \\
\hline
\end{tabular}

Figure 14: Total protein level: WT vs. PSD-95 KO

\begin{tabular}{|c|c|c|c|c|c|}
\hline $\begin{array}{l}\text { AFigure } \\
14\end{array}$ & $\mathrm{GABA}_{\alpha 1}$ & 2-way ANOVA & Genotype & $F_{g t(1,20)}=4.271$ & $p_{g t}=0.052$ \\
\hline & & & Age & $\mathrm{F}_{\mathrm{ag}(2,20)}=44.529$ & $p_{a g}<0.001$ \\
\hline & & & Interaction & $F_{g t x a g(2,20)}=3.106$ & $p_{\text {gtxag }}=0.067$ \\
\hline \multirow[t]{6}{*}{ B } & GluN2A & 2-way ANOVA & Genotype & $F_{g t(1,20)}=0.613$ & $p_{g t}=0.443$ \\
\hline & & & Age & $\mathrm{F}_{\mathrm{ag}(2,20)}=4.972$ & $p_{a g}=0.018$ \\
\hline & & & Interaction & $F_{\text {gtxag }(2,20)}=0.357$ & $p_{\text {gtxag }}=0.704$ \\
\hline & GluN2B & 2-way ANOVA & Genotype & $F_{g t(1,20)}=0.296$ & $\mathrm{p}_{\mathrm{gt}}=0.592$ \\
\hline & & & Age & $F_{\mathrm{ag}(2,20)}=3.455$ & $p_{a g}=0.051$ \\
\hline & & & Interaction & $F_{g t x a g(2,20)}=2.443$ & $p_{g t x a g}=0.112$ \\
\hline \multirow[t]{6}{*}{ C } & GluA1 & 2-way ANOVA & Genotype & $F_{g t(1,20)}=0.068$ & $\mathrm{p}_{\mathrm{gt}}=0.797$ \\
\hline & & & Age & $F_{a g(2,20)}=6.348$ & $p_{a g}=0.017$ \\
\hline & & & Interaction & $F_{\text {gtxag }(2,20)}=0.365$ & $p_{\text {gtxag }}=0.699$ \\
\hline & GluA2 & 2-way ANOVA & Genotype & $F_{g t(1,20)}=0.997$ & $p_{g t}=0.330$ \\
\hline & & & Age & $\mathrm{F}_{\mathrm{ag}(2,20)}=1.449$ & $p_{a g}=0.258$ \\
\hline & & & Interaction & $F_{\text {gtxag }(2,20)}=0.631$ & $p_{\text {gtxag }}=0.542$ \\
\hline
\end{tabular}

Figure 16: Protein surface level (P28-32)

\begin{tabular}{llllll} 
B & GluA1 & 1-way ANOVA & Genotype & $F_{g t(3,22)=1.341}$ & $p_{g t}=0.287$ \\
\hline & GluA2 & 1-way ANOVA & Genotype & $F_{g t(3,22)=0.348}$ & $p_{g t}=0.791$ \\
\hline C & GluN2A & 1-way ANOVA & Genotype & $F_{g t(3,22)}=1.770$ & $p_{g t}=0.182$ \\
& GluN2B & 1-way ANOVA & Genotype & $F_{g t(3,19)}=0.636$ & $p_{g t}=0.601$
\end{tabular}

Figure 17: Protein surface level: WT vs. PSD-95 KO

$\begin{array}{rlllll}\text { A } & \text { GluA1 } & \text { 2-way ANOVA } & \text { Genotype } & F_{\mathrm{gt}(1,24)=8.021} & p_{\mathrm{gt}}=0.009 \\ & & \text { Age } & \mathrm{F}_{\mathrm{ag}(2,24)=2.796} & p_{\mathrm{ag}}=0.081 \\ & & \text { Interaction } & \mathrm{F}_{\mathrm{gtxag}(2,24)=0.055} & p_{\mathrm{gtxag}}=0.946 \\ & & \mathrm{~F}_{\mathrm{ag}(2,10)=1.167} & p_{\mathrm{ag}}=0.350 \\ & \text { 1-way ANOVA } & \text { Age, WT } & \mathrm{F}_{\mathrm{ag}(2,14)=1.781} & p_{\mathrm{ag}}=0.205\end{array}$




\begin{tabular}{|c|c|c|c|c|c|}
\hline & \multirow[t]{11}{*}{ GluA2 } & \multirow[t]{3}{*}{ 2-way ANOVA } & \multirow{2}{*}{$\begin{array}{l}\text { Genotype } \\
\text { Age }\end{array}$} & \multirow{2}{*}{$\begin{array}{l}F_{g t(1,24)}=4.697 \\
F_{a g(2,24)}=31.256\end{array}$} & \multirow{2}{*}{$\begin{array}{l}p_{g t}=0.040 \\
p_{a g}<0.001\end{array}$} \\
\hline & & & & & \\
\hline & & & Interaction & $F_{\text {gtxag }(2,24)}=2.605$ & $p_{g t x a g}=0.095$ \\
\hline & & 1-way ANOVA & Age, WT & $\mathrm{F}_{\mathrm{ag}(2,10)}=32.085$ & $\mathrm{p}_{\mathrm{ag}}<0.001$ \\
\hline & & & P13-14 vs. P20-21 & & $p<0.001$ \\
\hline & & & P13-14 vs. P28-32 & & $p<0.001$ \\
\hline & & & P20-21 vs. P28-32 & & $p=0.087$ \\
\hline & & 1-way ANOVA & Age, PSD-95 KO & $F_{a g(2,14)}=9.267$ & $p_{a g}=0.003$ \\
\hline & & & P13-14 vs. P20-21 & & $p=0.010$ \\
\hline & & & P13-14 vs. P28-32 & & $p=0.001$ \\
\hline & & & P20-21 vs. P28-32 & & $p=0.523$ \\
\hline \multirow[t]{13}{*}{$B$} & GluN2A & 2-way ANOVA & Genotype & $F_{g t(1,23)}=1.121$ & $\mathrm{p}_{\mathrm{gt}}=0.301$ \\
\hline & & & Age & $F_{a g(2,23)}=0.550$ & $p_{a g}=0.585$ \\
\hline & & & Interaction & $F_{\text {gtxag }(2,23)}=5.898$ & pgtxag $=0.009$ \\
\hline & & 1-way ANOVA & Age, WT & $F_{a g}(2,10)=11.116$ & $p_{a g}=0.003$ \\
\hline & & & P13-14 vs. P20-21 & & $p=0.010$ \\
\hline & & & P13-14 vs. P28-32 & & $p=0.201$ \\
\hline & & & P20-21 vs. P28-32 & & $p=0.001$ \\
\hline & & 1-way ANOVA & Age, PSD-95 KO & $F_{a g(2,13)}=1.612$ & $p_{a g}=0.237$ \\
\hline & GluN2B & 2-way ANOVA & Genotype & $F_{g t(1,20)}=0.671$ & $p_{g t}=0.422$ \\
\hline & & & Age & $F_{a g(2,20)}=0.222$ & $p_{a g}=0.803$ \\
\hline & & & Interaction & $F_{g t x a g(2,20)}=0.109$ & $p_{g t x a g}=0.897$ \\
\hline & & 1-way ANOVA & Age, WT & $F_{a g(2,9)}=0.733$ & $\mathrm{p}_{\mathrm{ag}}=0.507$ \\
\hline & & 1-way ANOVA & Age, PSD-95 KO & $F_{a g}(2,11)=0.008$ & $p_{a g}=0.993$ \\
\hline \multirow[t]{8}{*}{ C } & $\mathrm{GABA}_{\alpha 1}$ & 2-way ANOVA & Genotype & $F_{g t(1,24)}=6.816$ & $p_{g t}=0.015$ \\
\hline & & & Age & $F_{a g(2,24)}=0.444$ & $\mathrm{p}_{\mathrm{ag}}=0.646$ \\
\hline & & & Interaction & $F_{\text {gtxag }(2,24)}=5.359$ & pgtxag $=0.012$ \\
\hline & & 1-way ANOVA & Age, WT & $F_{a g}(2,10)=4.207$ & $p_{a g}=0.047$ \\
\hline & & & P13-14 vs. P20-21 & & $p=0.027$ \\
\hline & & & P13-14 vs. P28-32 & & $p=0.857$ \\
\hline & & & P20-21 vs. P28-32 & & $p=0.025$ \\
\hline & & 1-way ANOVA & Age, PSD-95 KO & $F_{a g}(2,10)=2.204$ & $p_{a g}=0.147$ \\
\hline
\end{tabular}

Figure 20: Synaptotagmin 1 uptake assay

Two-tailed, unpaired Student's t-test

$\begin{array}{ll}\text { TCM vs. Control } & p=0.348 \\ \text { KCl vs. Control } & p=0.014 \\ \text { Sucrose vs. Control } & p=0.210\end{array}$




\section{ACKNOWLEDGEMENTS}

First and foremost, I would like to sincerely express my gratitude to my supervisor Prof. Dr. Dr. Oliver Schlüter who gave me the opportunity to perform my $\mathrm{PhD}$ in his lab. I am especially thankful for your scientific guidance, infectious optimism and remarkable enthusiasm throughout our meetings and discussions.

Furthermore, I am deeply thankful for the collaboration with Prof. Dr. Michael Müller. You provided a second 'lab family' to me and always spared some time to answer questions, help with technical problems or give valuable scientific advice.

I gratefully acknowledge Prof. Dr. Siegrid Löwel for her constructive feedback and precious scientific input during my thesis committee meetings. I really appreciate your time for being the second reviewer of my thesis.

I would also like to sincerely thank Prof. Dr. Dr. Hannelore Ehrenreich, Prof. Dr. Oliver Wirths and Prof. Dr. Ralf Heinrich for your agreement on being part of my examination board.

Many thanks go to the GAUSS Biology program of the University of Göttingen and to the GAUSS office, especially to Dr. Jonas Barth who always helped with administrative issues by providing immediate response.

$$
* * *
$$

I am deeply grateful for all the past and present members of the 'Molecular Neurobiology' lab. Many thanks go to our technician Sandra Ott-Gebauer - the 'good soul' of our group. You always had an open ear for every kind of issue and I hardly met a person with such a friendly and lovely character. Thank you for your technical advice and for taking care of us.

I owe special thanks to Dr. Lei Cui who always provided help and support. You are such a generous person and I wish you all the best for your new start.

I am as well very grateful for my dear lab colleagues Myrto and Alex. Myrto, thank you for lighting up the whole atmosphere with your overwhelming temperament and for being a good friend. Alex, thank you for teaching me the secret of patching and for our encouraging and fun conversations.

Moreover, I would like to deeply thank all the already and further mentioned lab members for creating such a nice and inspiring working environment: Yanling, Man Ho, Avani, Plinio, Lisa, Eli and Huang. 
Many thanks also go to the lab members of Prof. Michael Müller. I had such a great and enriching time joining your group and I am incredibly grateful for getting this opportunity. I would like to especially thank Dr. Katharina Dietrich for introducing me to the electrophysiological set-up and Belinda Kempkes for your technical help and support. Together with Karolina, Carina, Nina and Hendrik I couldn't have imagined a better place for collaboration.

\section{$* * *$}

Furthermore, I am so grateful for all the friends I made in Göttingen, especially to Beate who joined us by a happy coincidence and became a good friend right from the beginning. I am still very sad that our geographical ways were separated but hope we will stay in contact for a much longer time. Adriana, you are such a lovely person and I am so happy that I had the chance to meet you and spend time together. Maddy, our girl's nights were always so much fun! Please keep your creativity and uniqueness.

Many thanks go to my former fellow students from Hannover - studies would not have been the same without you. I hope we will always be the 'Freunde der Ferne'. Kristina, amidst this stressful PhD life we spent an amazing holiday together which I will never forget. Anna, I am sure I would have been desperate in some situations if I couldn't have talked to you. I am so grateful that we stayed in touch and wish that we will ever be part of each other's life - there is always a road to beautiful North coast ;).

$$
* * *
$$

Of course, I would also like to thank some of my closest friends: Lea, Leni, Kerstin and Rile. You know that you are such special persons to me and I really appreciate our long friendship. I enjoyed every girl's weekend with you to the fullest - it is like coming home. I hope we will maintain this connection forever.

Lisa, I met you right after moving to Göttingen and I would never want to miss you again. You cheered up my time in the lab so much - thank you for all your emotional support and for bringing me chocolate when I needed it the most. And thank you, that our friendship became even closer afterwards.

Melli, I am impressed of your positive and lovely personality - you always find a reason to smile and cheer your friends up. Thank you for our sunny lunch breaks together and for every wine-loaded 'therapy evening' we had ;). 
Meiki, you know what you mean to me. You are the best and longest friend I ever had. We never lost our connection and I know we never will - no matter the distance which might appear. Thank you for constantly being there for me, listening to everything and having the funniest nights-out together. No words can express how thankful I am for our friendship.

$$
* * *
$$

I also want to give heartfelt thanks to my boyfriend Morris who accompanied me during the past years. Despite the large geographical distance, you were always there for me and encouraged me in the hardest times. You know better than everyone else how to make me smile and laugh. Life is so much more having you by my side. Thank you for finding me.

My biggest thanks go to my beloved family who made my whole studies in science possible and always welcome me with wide open arms - no matter what. Thank you, mum, dad, my brother and grandparents. Thank you so much for all your support and trust, endless love and care. You are the most valuable gift in the world. 
